Supporting Information

for

\title{
Electronic Structure and Excited-State Dynamics of Rylene-Tetrapyrrole Panchromatic Absorbers
}

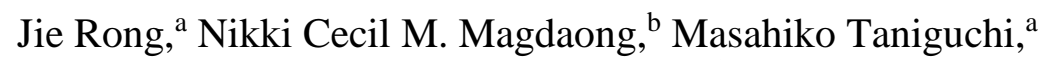
James R. Diers, ${ }^{c}$ Dariusz M. Niedzwiedzki, ${ }^{\mathrm{d}}$ Christine Kirmaier, ${ }^{\mathrm{b}}$ Jonathan S. Lindsey, ${ }^{*, a}$ David F. Bocian, ${ }^{*, c}$ and Dewey Holten*,

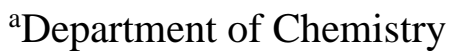

North Carolina State University

Raleigh, North Carolina 27695-8204, USA

${ }^{\mathrm{b}}$ Department of Chemistry

Washington University

St. Louis, Missouri 63130-4889, USA

${ }^{\mathrm{c}}$ Department of Chemistry

University of California

Riverside, California 92521-0403, USA

${ }^{\mathrm{d} C e n t e r}$ for Solar Energy and Energy Storage, and

Department of Energy, Environmental \& Chemical Engineering

Washington University

St. Louis, MO 63130-4889, USA 


\section{Table of Contents}

Item

Section S1.

Topic S1-1.

Figure S1.

Figure S2.

Figure S3.

Figure S4.

Figure S5.

Figure S6.

Figure S7.

Figure S8.

Figure S9.

Figure S10.

Figure S11.

Figure S12.

Figure S13.

Figure S14.

Figure S15.

Figure S16.

Figure S17.

Figure S18.

Figure S19.

Figure S20.

Figure S21.

Figure S22.

Figure S23.

Topic S1-2.

Figure S24.

Figure S25.

Figure S26.

Figure S27.

Figure S28.

Section S2.

Table S1.

Figure S29.

Section S3.

Table S2.

Figure S30.

Figure S31.

Figure S32.

Figure S33.

Figure S34.

Figure S35.

Figure S36.

Figure S37.

Figure S38.
Synthesis

Page

Synthesis procedures and characterization data

MALDI-MS data of compound TMI-e .................................................... S10

${ }^{1}$ H NMR spectrum of compound TMI-e .................................................. S1

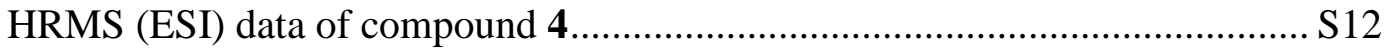

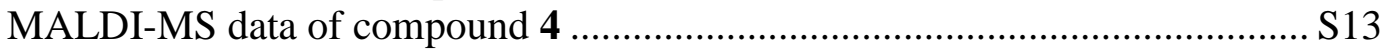

${ }^{1}$ H NMR spectrum of compound 4 ...................................................... S14

HRMS (ESI) data of compound 5........................................................ S15

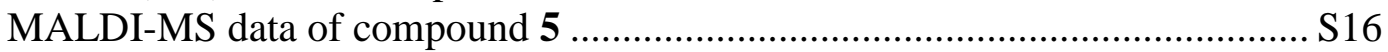

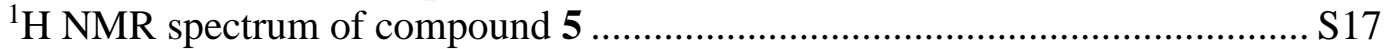

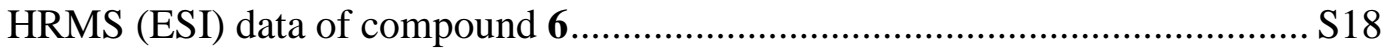

MALDI-MS data of compound 6 ............................................................ S19

${ }^{1} \mathrm{H}$ NMR spectrum of compound 6 ....................................................... S20

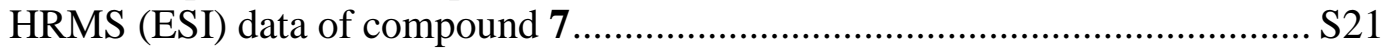

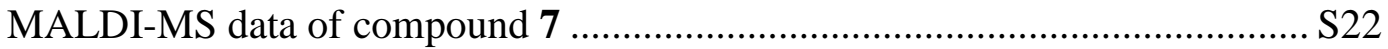

${ }^{1} \mathrm{H}$ NMR spectrum of compound 7 ........................................................ S23

HRMS (ESI) data of compound PMI-P-TMI.......................................... S24

MALDI-MS data of compound PMI-P-TMI............................................ S25

${ }^{1}$ H NMR spectrum of compound PMI-P-TMI ......................................... S26

HRMS (ESI) data of compound B-TMI.................................................... S27

MALDI-MS data of compound B-TMI ................................................. S28

${ }^{1} \mathrm{H}$ NMR spectrum of compound B-TMI ................................................ S29

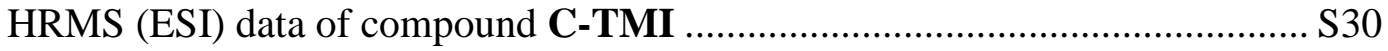

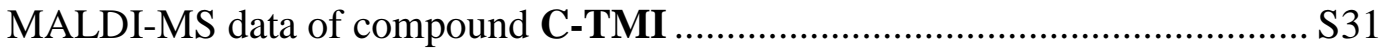

${ }^{1} \mathrm{H}$ NMR spectrum of compound C-TMI ................................................ S32

Analytical SEC monitoring of the formation of PMI-P-TMI triad .................. S33

Analytical SEC traces for the formation of PMI-P-TMI triad ....................... S33

Analytical SEC trace of compound PMI-P-TMI........................................ S34

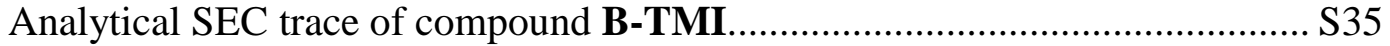

Analytical SEC trace of compound C-TMI .......................................... S36

Absorption spectra for compounds TMI-I and TMI-e ............................... S37

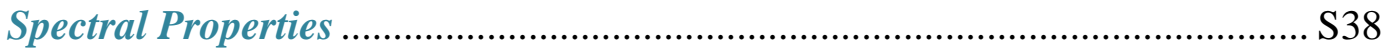

Spectral properties of arrays and benchmark monomers in benzonitrile........... S39

Absorption and fluorescence spectra of arrays in benzonitrile ........................ S40

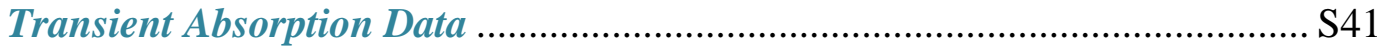

Summary of TA kinetic data for arrays from global analysis......................... S42

TA comparison for P-TMI, PMI-P-TMI, and B-TMI in benzonitrile ............ S43

TA data for P-TMI in toluene .................................................................. S44

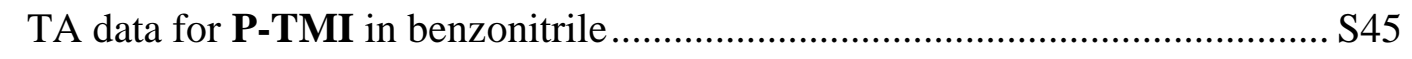

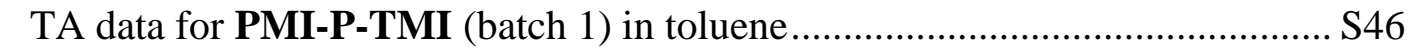

TA data for PMI-P-TMI (batch 2) in toluene............................................... S47

TA data for PMI-P-TMI (batch 1) in benzonitrile ....................................... S48

TA data for PMI-P-TMI (batch 2) in benzonitrile ...................................... S49

TA data for PMI-P-TMI (batch 1) at different temperatures .......................... S50

S51 


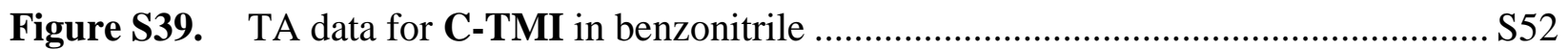

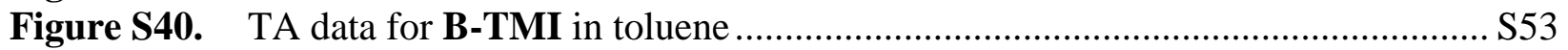

Figure S41. TA data for B-TMI in benzonitrile.............................................................. S54

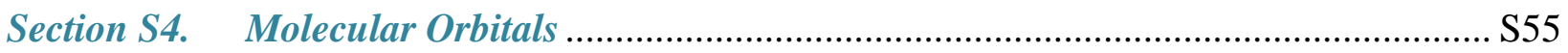

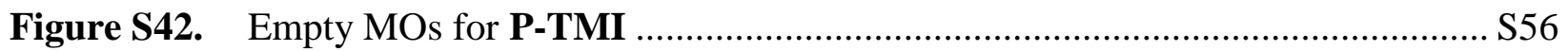

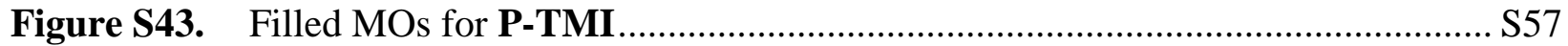

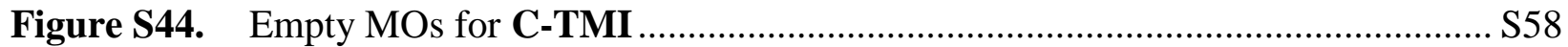

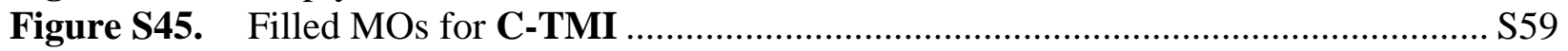

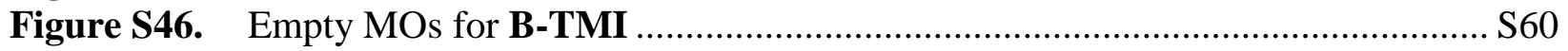

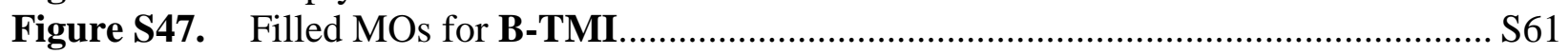

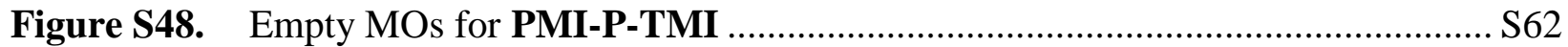

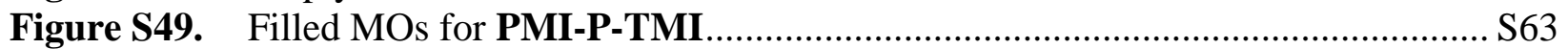

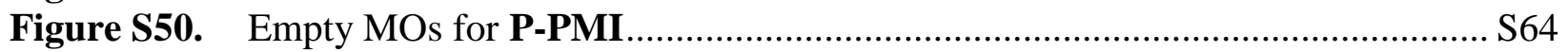

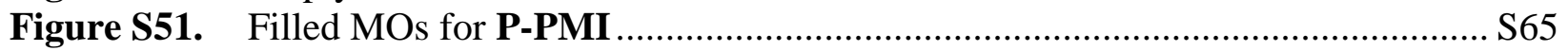

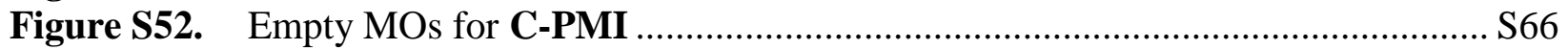

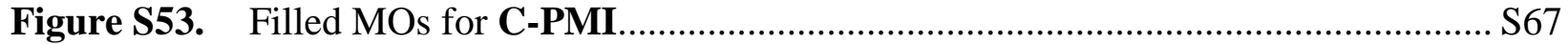

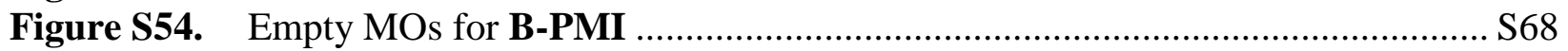

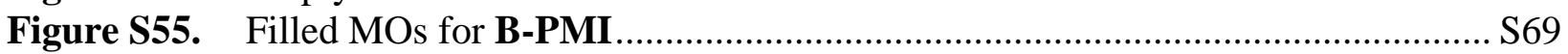

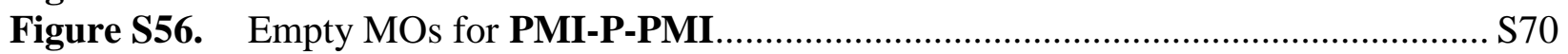

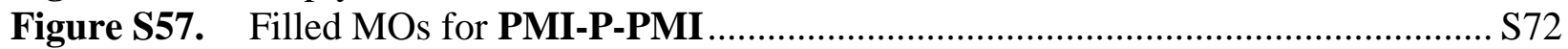

Section S5. Molecular Orbital Correlation Diagrams ………....................................... S74

Figure S58. MO correlation diagram for P-TMI ………................................................ S75

Figure S59. MO correlation diagram for C-TMI ……...................................................... 576

Figure S60. MO correlation diagram for B-TMI ……….............................................. S77

Figure S61. MO correlation diagram for PMI-P-TMI .............................................. S78

Figure S62. MO correlation diagram for P-PMI ………................................................ S79

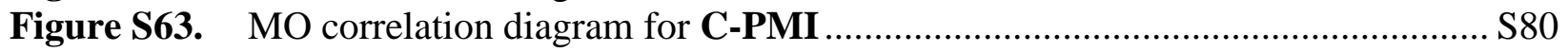

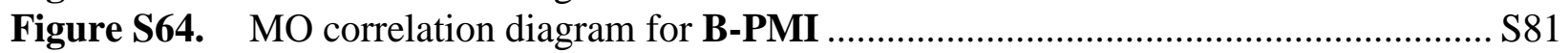

Figure S65. MO correlation diagram for PMI-P-PMI ...................................................... S82

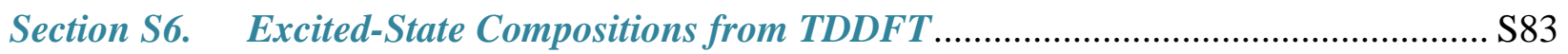

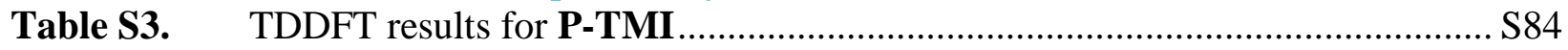

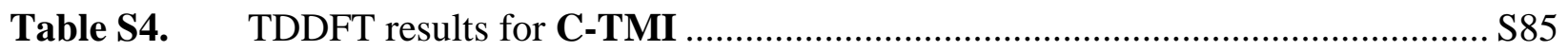

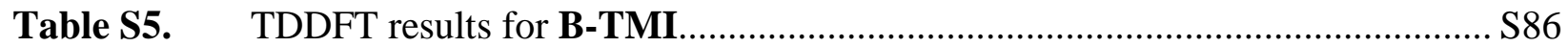

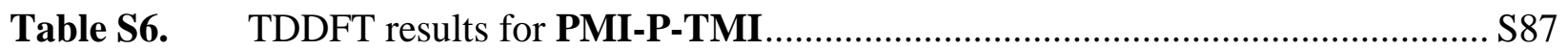

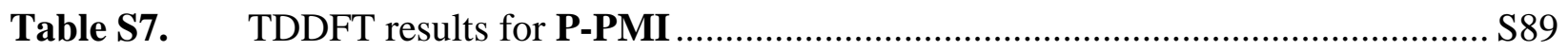

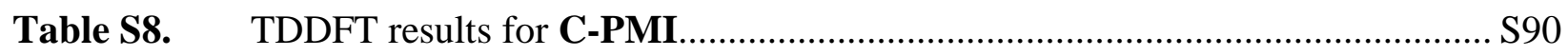

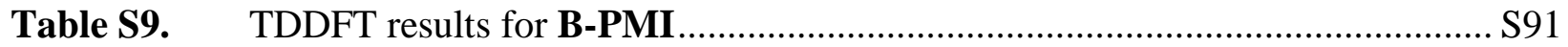

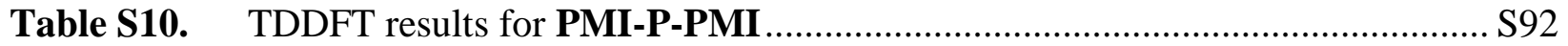

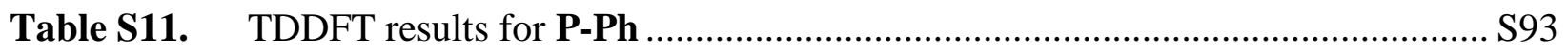

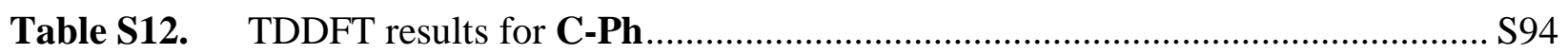

Table S13. TDDFT results for B-Ph ............................................................................ S95

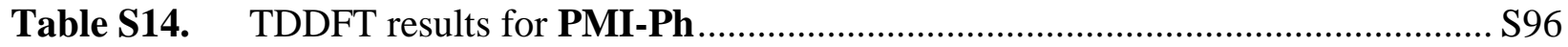

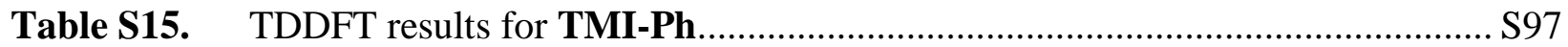

Table S16. TDDFT results for PMI-P-PMI …………..................................................... 99 
Section S7. Experimental vs Calculated Absorption Spectra and NTOs ....................... S99

Figure S66. Comparison of spectra and NTOs for $S_{1}$ and $S_{2}$ of P-TMI ......................... S100

Figure S67. Comparison of spectra and NTOs for $S_{3}$ of P-TMI …............................... S101

Figure S68. Comparison of spectra and NTOs for $\mathrm{S}_{4}$ and $\mathrm{S}_{5}$ of P-TMI ......................... S102

Figure S69. Comparison of spectra and NTOs for $S_{1}$ and $S_{2}$ of $\mathbf{C}$-TMI ......................... S103

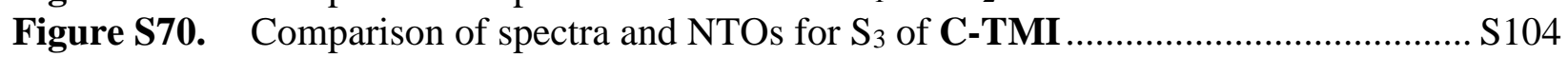

Figure S71. Comparison of spectra and NTOs for $\mathrm{S}_{4}$ and $\mathrm{S}_{5}$ of C-TMI ......................... S105

Figure S72. Comparison of spectra and NTOs for $S_{1}$ and $S_{2}$ of B-TMI ......................... S106

Figure S73. Comparison of spectra and NTOs for $S_{3}$ of B-TMI .................................. S107

Figure S74. Comparison of spectra and NTOs for $\mathrm{S}_{4}$ and $\mathrm{S}_{10}$ of B-TMI ......................... S108

Figure S75. Comparison of spectra and NTOs for $S_{1}$ and $S_{2}$ of PMI-P-TMI ................... S109

Figure S76. Comparison of spectra and NTOs for $S_{3}$ and $S_{4}$ of PMI-P-TMI ................... S110

Figure S77. Comparison of spectra and NTOs for $S_{5}$ and $S_{6}$ of PMI-P-TMI .................. S111

Figure S78. Comparison of spectra and NTOs for $S_{1}$ and $S_{2}$ of P-PMI ......................... S112

Figure S79. Comparison of spectra and NTOs for $\mathrm{S}_{3}$ of P-PMI ................................... S113

Figure S80. Comparison of spectra and NTOs for $S_{4}$ and $S_{5}$ of P-PMI .......................... S114

Figure S81. Comparison of spectra and NTOs for $S_{1}$ and $S_{2}$ of C-PMI.......................... S115

Figure S82. Comparison of spectra and NTOs for $\mathrm{S}_{3}$ of C-PMI.................................... S116

Figure S83. Comparison of spectra and NTOs for $\mathrm{S}_{4}$ and $\mathrm{S}_{5}$ of $\mathbf{C}$-PMI............................ S117

Figure S84. Comparison of spectra and NTOs for $S_{1}$ and $S_{2}$ of B-PMI.......................... S118

Figure S85. Comparison of spectra and NTOs for $S_{3}$ of B-PMI................................... S119

Figure S86. Comparison of spectra and NTOs for $S_{4}$ and $S_{5}$ of B-PMI......................... S120

Figure S87. Comparison of spectra and NTOs for $S_{1}$ and $S_{2}$ of $\mathbf{P}-\mathbf{P h}$............................. $S 121$

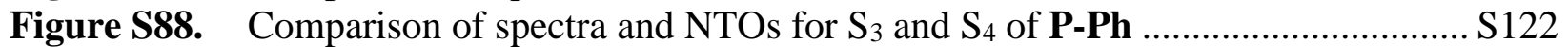

Figure S89. Comparison of spectra and NTOs for $S_{1}$ and $S_{2}$ of $\mathbf{C - P h ~ . . . . . . . . . . . . . . . . . . . . . . . . . . . ~ S 1 2 3 ~}$

Figure S90. Comparison of spectra and NTOs for $\mathrm{S}_{3}$ and $\mathrm{S}_{4}$ of $\mathbf{C}-\mathbf{P h}$........................... S124

Figure S91. Comparison of spectra and NTOs for $S_{1}$ and $S_{2}$ of B-Ph .......................... S125

Figure S92. Comparison of spectra and NTOs for $S_{3}$ and $S_{4}$ of B-Ph ...........................S126

Figure S93. Comparison of spectra and NTOs for $S_{1}$ and $S_{2}$ of TMI-Ph........................ S127

Figure S94. Comparison of spectra and NTOs for $S_{1}$ and $S_{2}$ of PMI-Ph ....................... S128

Figure S95. Comparison of spectra and NTOs for $S_{1}$ and $S_{2}$ of PMI-P-PMI................... S129

Figure S96. Comparison of spectra and NTOs for $S_{3}$ and $S_{4}$ of PMI-P-PMI .................. S130

Figure S97. Comparison of spectra and NTOs for $\mathrm{S}_{5}$ and $\mathrm{S}_{6}$ of PMI-P-PMI.................... S131

Section S8. Calculated Permanent Dipoles ................................................................ S132

Figure S98. Permanent Dipole of PMI-P-TMI in the ground state................................ S133

Figure S99. Permanent Dipole of PMI-P-TMI in $\mathrm{S}_{1}$ at the $\mathrm{S}_{0}$ geometry ......................... S134

Figure S100. Permanent Dipole of PMI-P-PMI in the ground state ................................ S135

Figure S101. Permanent Dipole of PMI-P-PMI in $S_{1}$ at the $S_{0}$ geometry ....................... S136

Section S9. Full References for Citations Truncated after the First 10 Authors ............ S137

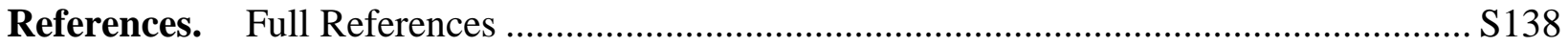


Section S1

Synthesis 


\section{Topic S1. Synthesis Procedures and Characterization Data.}

Compound PMI-Br was prepared following a literature procedure. ${ }^{16}$ TMI-I and TMI-e building blocks were prepared following literature procedures ${ }^{16}$ but at much larger scale. A Suzuki coupling of PMI-Br with (5-nitronaphthalen-1-yl)boronic acid pinacol ester afforded the perylene-naphthalene (1) in $92 \%$ yield (Scheme 1). Ring closure of compound $\mathbf{1}$ afforded nitroterrylene monoimide (2) in $30 \%$ yield. Reduction of the nitro group to the amine with $\mathrm{H}_{2}$ and $\mathrm{Pd} / \mathrm{C}$, diazotization of the resulting amine, and Sandmeyer reaction afforded the iodo-terrylene monoimide (TMI-I) building block in $11 \%$ yield. Sonogashira coupling of TMI-I and triisopropylsilylacetylene in the presence of $\mathrm{CuI}$ followed by deprotection of the triisopropylsilyl group afforded the TMI-e building block in $44 \%$ yield. Approximately $25 \mathrm{mg}$ of TMI-e building block was prepared in one batch (versus $3.2 \mathrm{mg}$ previously ${ }^{16}$ ).

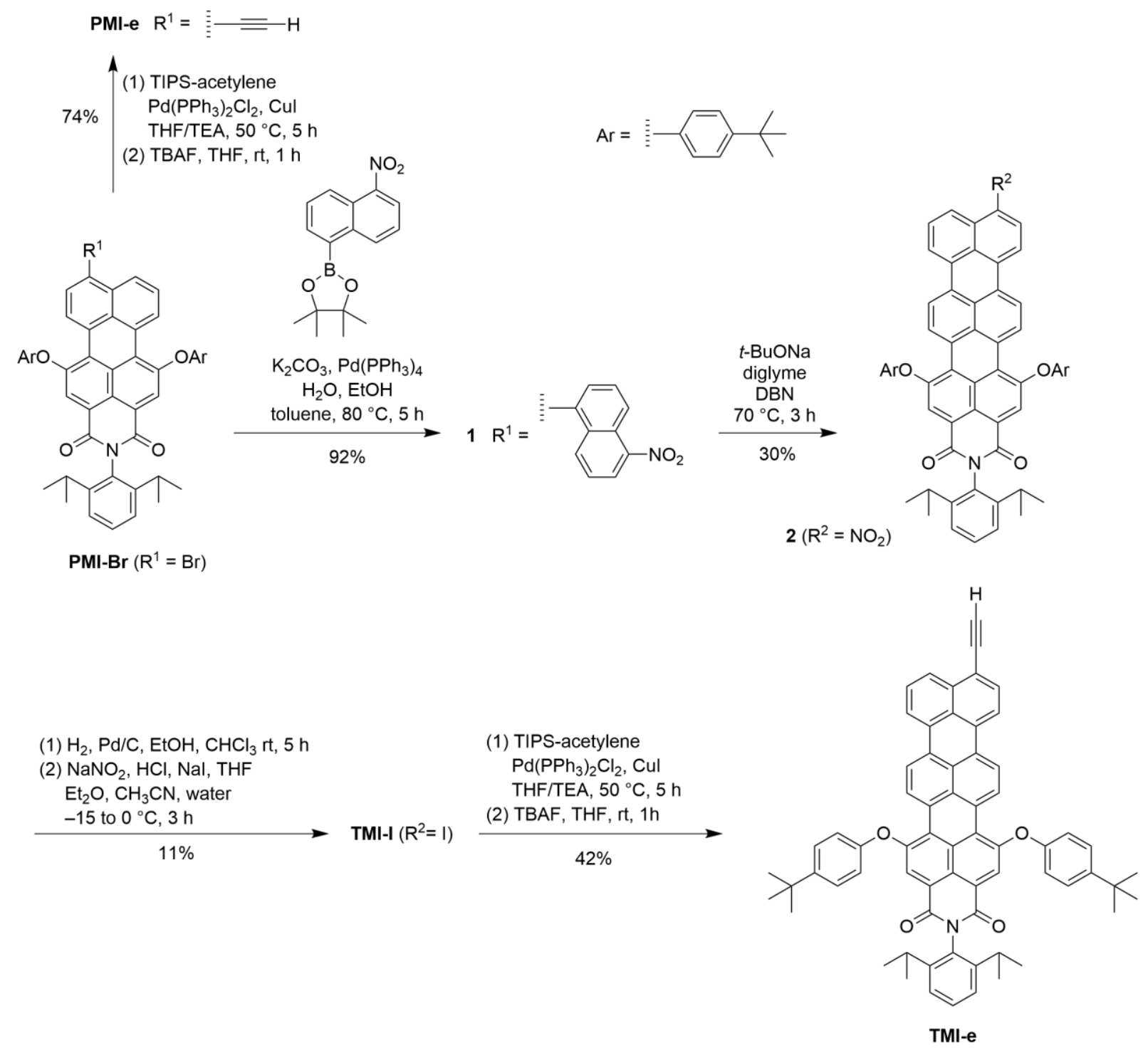

Scheme S1. Synthesis of perylene and terrylene building blocks.

\section{General methods}

All commercial chemicals were used as received. THF was freshly distilled from sodium/benzophenone ketyl and used immediately. Chloroform contained approximately $0.75 \%$ ethanol as preservative. Noncommercial compounds 5-(2-(triisopropylsilyl)ethynyl)-10,20- 
diphenylporphinato zinc(II) (3), ${ }^{27,28}$ PMI-Br, ${ }^{16}$ PMI-e, ${ }^{16}$ TMI-I,${ }^{16}$ TMI-e, ${ }^{16} \mathbf{C}^{6} \mathbf{~ B r}^{32}$ and B-Br ${ }^{33}$ were prepared following literature procedures. MALDI-MS was performed using $\alpha$-cyano-4hydroxycinnamic acid ( $\alpha-\mathrm{CHCA})$ as matrix.

\section{Chromatography}

Following an established method, ${ }^{14,31,34}$ a three-chromatography procedure was used for purification of arrays. The Pd-coupling reaction mixture was chromatographed with (i) silica column chromatography to remove catalysts and ligands from the coupling reaction, (ii) preparative-scale size exclusion chromatography (SEC) using BioRad Bio-Beads SX-1 (toluene, $0.2 \mathrm{~mL} / \mathrm{min}$ ) to effect separation on the basis of molecular size, and (iii) silica column chromatography with $\mathrm{CH}_{2} \mathrm{Cl}_{2}$ and distilled hexanes to complete final purification.

Analytical SEC was performed to monitor the reactions and purification of the samples. ${ }^{14,34}$ Analytical SEC columns (styrene-divinylbenzene copolymer) were purchased from Polymer Laboratories. Analytical SEC was performed with an Agilent 1100 HPLC using PLgel $50 \AA$, PLgel $100 \AA$ and PLgel $500 \AA$ in series eluting with toluene (flow rate $=0.8 \mathrm{~mL} / \mathrm{min}$ ) at room temperature. Sample detection was achieved by absorption spectroscopy using a diode array detector with quantitation at 440, 630, 700, $750 \mathrm{~nm}$ for PMI-P-TMI triad; 412, 672 and $705 \mathrm{~nm}$ for C-TMI dyad; and 383, 685, $774 \mathrm{~nm}$ for B-TMI dyad ( $\pm 8 \mathrm{~nm}$ bandwidth), which best captures the peaks of the various arrays.

\section{Procedures and Chemical Characteristics \\ $N$-(2,6-Diisopropylphenyl)-1,6-bis(4-tert-butylphenoxy)-11-ethynylterrylene-3,4-}

dicarboximide (TMI-e). Following a standard procedure, ${ }^{16,30,31}$ a Schlenk flask containing TMII (66 mg, $64 \mu \mathrm{mol})$, triisopropylsilylacetylene (35 mg, $192 \mu \mathrm{mol})$, and CuI (3.8 mg, $20 \mu \mathrm{mol})$ was flushed with argon, treated with degassed toluene/TEA $(10 \mathrm{~mL}, 5: 1)$ and subjected to three freezepump-thaw cycles, then treated with $\mathrm{Pd}\left(\mathrm{PPh}_{3}\right)_{2} \mathrm{Cl}_{2}(14 \mathrm{mg}, 20 \mu \mathrm{mol})$ and subjected to two additional freeze-pump-thaw cycles. The resulting mixture was heated to $60{ }^{\circ} \mathrm{C}$ for $16 \mathrm{~h}$. The crude mixture was allowed to cool to room temperature and then concentrated to dryness. The resulting mixture was dissolved in THF $(10 \mathrm{~mL})$ and treated with tetrabutylammonium fluoride solution $(400 \mu \mathrm{L}, 1.0 \mathrm{M}$ in $\mathrm{THF})$ at room temperature for $1.5 \mathrm{~h}$. The mixture was diluted with $\mathrm{CH}_{2} \mathrm{Cl}_{2}(40 \mathrm{~mL})$, washed with water $(40 \mathrm{~mL})$ and brine $(40 \mathrm{~mL})$, dried, concentrated to dryness and then purified by chromatography [silica, hexanes $/ \mathrm{CH}_{2} \mathrm{Cl}_{2}(3: 1$ to $1: 1)$ ] to afford a blue solid (25 mg, 42\%). ${ }^{1} \mathrm{H}$ NMR (600 MHz, $\left.\mathrm{CDCl}_{3}\right) \delta 9.43-9.35(\mathrm{~m}, 2 \mathrm{H}), 8.36-8.28(\mathrm{~m}, 4 \mathrm{H}), 8.26(\mathrm{~d}, J=$ $8.2 \mathrm{~Hz}, 1 \mathrm{H}), 8.19(\mathrm{~d}, J=7.9 \mathrm{~Hz}, 1 \mathrm{H}), 7.77-7.73(\mathrm{~m}, 1 \mathrm{H}), 7.61(\mathrm{t}, J=7.9 \mathrm{~Hz}, 1 \mathrm{H}), 7.56-7.52(\mathrm{~m}$, $1 \mathrm{H}), 7.46-7.39(\mathrm{~m}, 5 \mathrm{H}), 7.30(\mathrm{~d}, J=7.9 \mathrm{~Hz}, 2 \mathrm{H}), 7.13-7.05(\mathrm{~m}, 4 \mathrm{H}), 3.60(\mathrm{~s}, 1 \mathrm{H}), 2.73(\mathrm{p}, J=6.8$ $\mathrm{Hz}, 2 \mathrm{H}), 1.33(\mathrm{~s}, 18 \mathrm{H}), 1.15(\mathrm{~d}, J=6.8 \mathrm{~Hz}, 12 \mathrm{H}) ; \lambda_{\text {abs }}$ (toluene) $648 \mathrm{~nm}$. Calcd 925.4143 for $\mathrm{C}_{66} \mathrm{H}_{55} \mathrm{NO}_{4}\left(\mathrm{M}^{+}\right)$, MALDI-MS found $924.752\left(\mathrm{M}-\mathrm{H}^{+}\right)$.

5-Bromo-15-(2-triisopropylsilyl)ethynyl)-10,20-diphenylporphinato zinc(II) (4). A solution of 3 (38 mg, $54 \mu \mathrm{mol})$ in $\mathrm{CH}_{2} \mathrm{Cl}_{2}$ :pyridine $(8 \mathrm{~mL}, 95: 5)$ was treated with NBS (9.6 mg, $54 \mu \mathrm{mol})$ at $0{ }^{\circ} \mathrm{C}$ for $30 \mathrm{~min}$. The reaction was quenched by the addition of acetone $(2 \mathrm{~mL})$. The mixture was diluted with $\mathrm{CH}_{2} \mathrm{Cl}_{2}(20 \mathrm{~mL})$, washed with water $(20 \mathrm{~mL})$ and brine $(20 \mathrm{~mL})$, dried, concentrated to dryness and then purified by chromatography [silica, hexanes/ $\mathrm{CH}_{2} \mathrm{Cl}_{2}(4: 1$ to 1:1)] to afford a green solid (34 mg, 48\%): ${ }^{1} \mathrm{H}$ NMR (600 MHz, $\left.\mathrm{CDCl}_{3}\right) \delta 1.39-1.46(\mathrm{~m}, 21 \mathrm{H}), 7.73-$ $7.81(\mathrm{~m}, 6 \mathrm{H}), 8.13-8.19(\mathrm{~m}, 4 \mathrm{H}), 8.91(\mathrm{~d}, J=4.6 \mathrm{~Hz}, 2 \mathrm{H}), 8.94(\mathrm{~d}, J=4.5 \mathrm{~Hz}, 2 \mathrm{H}), 9.70(\mathrm{~d}, J=$ $4.6 \mathrm{~Hz}, 2 \mathrm{H}), 9.77(\mathrm{~d}, J=4.5 \mathrm{~Hz}, 2 \mathrm{H}) ; \lambda_{\text {abs }}\left(\mathrm{CHCl}_{3}\right) 429,565,607 \mathrm{~nm}$. HRMS (ESI) for $\mathrm{C}_{43} \mathrm{H}_{39} \mathrm{BrN}_{4} \mathrm{SiZn}\left(\mathrm{M}+\mathrm{H}^{+}\right)$, calcd 783.1492, found 783.1465; MALDI-MS found 784.367. 
5-Bromo-15-(2-triisopropylsilyl)ethynyl)-10,20-diphenylporphyrin (5). A solution of $4(34 \mathrm{mg}, 43 \mu \mathrm{mol})$ in $\mathrm{CH}_{2} \mathrm{Cl}_{2}(15 \mathrm{~mL})$ was treated with TFA $(160 \mu \mathrm{L})$ at room temperature for 30 min. The reaction was diluted with $\mathrm{CH}_{2} \mathrm{Cl}_{2}(15 \mathrm{~mL})$, washed with saturated $\mathrm{NaHCO}_{3}$ aqueous solution $(20 \mathrm{~mL} \times 3)$, dried, concentrated to dryness and then purified by chromatography [silica, hexanes/ $\mathrm{CH}_{2} \mathrm{Cl}_{2}$ (4:1 to 1:1)] to afford a purple solid (27 mg, 87\%): ${ }^{1} \mathrm{H} \mathrm{NMR}\left(500 \mathrm{MHz}, \mathrm{CDCl}_{3}\right.$ ) $\delta-2.41(\mathrm{~s}, 2 \mathrm{H}), 1.36-1.44(\mathrm{~m}, 21 \mathrm{H}), 7.70-7.82(\mathrm{~m}, 6 \mathrm{H}), 8.10-8.20(\mathrm{~m}, 4 \mathrm{H}), 8.80(\mathrm{~d}, J=4.8 \mathrm{~Hz}$, $2 \mathrm{H}), 8.83(\mathrm{~d}, J=4.7 \mathrm{~Hz}, 2 \mathrm{H}), 9.58(\mathrm{~d}, J=4.8 \mathrm{~Hz}, 2 \mathrm{H}), 9.66(\mathrm{~d}, J=4.7 \mathrm{~Hz}, 2 \mathrm{H}) ; \lambda_{\text {abs }}\left(\mathrm{CHCl}_{3}\right) 427$, 532, 569, $670 \mathrm{~nm}$. HRMS (ESI) for $\mathrm{C}_{43} \mathrm{H}_{41} \mathrm{BrN}_{4} \mathrm{Si}\left(\mathrm{M}+\mathrm{H}^{+}\right)$, calcd 721.2357, found 721.2355; MALDI-MS found 722.407.

5-[N-(2,6-Diisopropylphenyl)-1,6-bis(4-tert-butylphenoxy)perylene-3,4dicarboximid-9-yl] ethynyl-15-(2-(triisopropylsilyl)ethynyl)-10,20-diphenylporphyrin (6). Following a standard method, ${ }^{16,30,31}$ a Schlenk flask containing 5 (27 mg, 37 mol), PMI-e (36 $\mathrm{mg}, 45 \mu \mathrm{mol})$ and tri $(o$-tolyl)phosphine $(14 \mathrm{mg}, 45 \mu \mathrm{mol})$ was flushed with argon, treated with degassed toluene/TEA ( $10 \mathrm{~mL}, 5: 1)$ and subjected to three freeze-pump-thaw cycles, then treated with tris(dibenzylideneacetone) dipalladium(0) $(10 \mathrm{mg}, 11 \mu \mathrm{mol})$ and subjected to two additional freeze-pump-thaw cycles. The resulting mixture was heated to $60{ }^{\circ} \mathrm{C}$ for $4 \mathrm{~h}$. The crude mixture was purified following the general procedure [silica (hexanes/ $\mathrm{CH}_{2} \mathrm{Cl}_{2}$ (2:1 to 1:1)); $\mathrm{SEC}$ (toluene); and silica (hexanes to hexanes/ $\left.\left.\mathrm{CH}_{2} \mathrm{Cl}_{2}(1: 1)\right)\right]$ to afford a green solid (15 mg, 52\%): ${ }^{1} \mathrm{H}$ NMR (600 $\left.\mathrm{MHz}, \mathrm{CDCl}_{3}\right) \delta-1.95(\mathrm{~s}, 2 \mathrm{H}), 1.16(\mathrm{~d}, J=6.8 \mathrm{~Hz}, 12 \mathrm{H}), 1.35(\mathrm{~s}, 9 \mathrm{H}), 1.35(\mathrm{~s}, 9 \mathrm{H}), 1.40-1.51(\mathrm{~m}$, 21H), 2.74 (pent, $J=6.8 \mathrm{~Hz}, 2 \mathrm{H}), 7.11-7.15(\mathrm{~m}, 4 \mathrm{H}), 7.31(\mathrm{~d}, J=7.9 \mathrm{~Hz}, 2 \mathrm{H}), 7.40-7.47(\mathrm{~m}, 5 \mathrm{H})$, 7.73-7.82 (m, 6H), $7.87(\mathrm{t}, J=8.0 \mathrm{~Hz}, 1 \mathrm{H}), 8.16-8.21(\mathrm{~m}, 4 \mathrm{H}), 8.32(\mathrm{~d}, J=8.0 \mathrm{~Hz}, 1 \mathrm{H}), 8.38(\mathrm{~s}$, $1 \mathrm{H}), 8.38(\mathrm{~s}, 1 \mathrm{H}), 8.83(\mathrm{~d}, J=4.6 \mathrm{~Hz}, 2 \mathrm{H}), 8.88(\mathrm{~d}, J=4.6 \mathrm{~Hz}, 2 \mathrm{H}), 9.11(\mathrm{~d}, J=8.0 \mathrm{~Hz}, 1 \mathrm{H}), 9.48-$ $9.53(\mathrm{~m}, 2 \mathrm{H}), 9.65(\mathrm{~d}, J=4.6 \mathrm{~Hz}, 2 \mathrm{H}), 9.77(\mathrm{~d}, J=4.6 \mathrm{~Hz}, 2 \mathrm{H}) ; \lambda_{\text {abs }}$ (toluene) 436, 536, 624, 703 nm. HRMS (ESI) for $\mathrm{C}_{99} \mathrm{H}_{91} \mathrm{~N}_{5} \mathrm{O}_{4} \mathrm{Si}\left(\mathrm{M}^{+}\right)$, calcd 1441.6835, found 1441.6814; MALDI-MS found $1442.822\left(\mathrm{M}+\mathrm{H}^{+}\right)$.

5-[N-(2,6-Diisopropylphenyl)-1,6-bis(4-tert-butylphenoxy)perylene-3,4dicarboximid-9-yl]ethynyl-15-ethynyl-10,20-diphenylporphyrin (7). A solution of 6 (20 mg, $14 \mu \mathrm{mol})$ in THF $(10 \mathrm{~mL})$ was treated with tetrabutylammonium fluoride solution $(100 \mu \mathrm{L}, 1.0 \mathrm{M}$ in THF) at room temperature for $1.5 \mathrm{~h}$. The reaction mixture was diluted with $\mathrm{CH}_{2} \mathrm{Cl}_{2}(30 \mathrm{~mL})$, washed with water $(20 \mathrm{~mL} \times 2)$, brine $(20 \mathrm{~mL})$, dried, concentrated, and purified by chromatography [silica, hexanes/ $\mathrm{CH}_{2} \mathrm{Cl}_{2}$ (4:1 to 1:1) to afford a green solid $(10 \mathrm{mg}, 56 \%):{ }^{1} \mathrm{H}$ $\operatorname{NMR}\left(600 \mathrm{MHz}, \mathrm{CDCl}_{3}\right) \delta-2.13(\mathrm{~s}, 2 \mathrm{H}), 1.18(\mathrm{~d}, J=6.8 \mathrm{~Hz}, 12 \mathrm{H}), 1.35(\mathrm{~s}, 9 \mathrm{H}), 1.36(\mathrm{~s}, 9 \mathrm{H})$, 2.77 (pent, $J=6.8 \mathrm{~Hz}, 2 \mathrm{H}), 7.07-7.10(\mathrm{~m}, 2 \mathrm{H}), 7.10-7.14(\mathrm{~m}, 2 \mathrm{H}), 7.32(\mathrm{~d}, J=8.0 \mathrm{~Hz}, 2 \mathrm{H}), 7.39$ $7.48(\mathrm{~m}, 5 \mathrm{H}), 7.72(\mathrm{t}, J=7.6 \mathrm{~Hz}, 1 \mathrm{H}), 7.75-7.87(\mathrm{~m}, 6 \mathrm{H}), 8.05(\mathrm{~d}, J=8.0 \mathrm{~Hz}, 1 \mathrm{H}), 8.17-8.26(\mathrm{~m}$, $4 \mathrm{H}), 8.36(\mathrm{~s}, 1 \mathrm{H}), 8.37(\mathrm{~s}, 1 \mathrm{H}), 8.83(\mathrm{~d}, J=4.6 \mathrm{~Hz}, 2 \mathrm{H}), 8.87-8.93(\mathrm{~m}, 3 \mathrm{H}), 9.21(\mathrm{~d}, J=8.0 \mathrm{~Hz}$, $1 \mathrm{H}), 9.34(\mathrm{~d}, J=7.6 \mathrm{~Hz}, 1 \mathrm{H}), 9.58(\mathrm{~d}, J=4.6 \mathrm{~Hz}, 2 \mathrm{H}), 9.78(\mathrm{~d}, J=4.6 \mathrm{~Hz}, 2 \mathrm{H}) ; \lambda_{\mathrm{abs}}$ (toluene) 437, $535,620,700 \mathrm{~nm} ; \lambda_{\text {em }}\left(\lambda_{\text {exc }}=534 \mathrm{~nm}\right) 703,775 \mathrm{~nm}$. HRMS (ESI) for $\mathrm{C}_{90} \mathrm{H}_{71} \mathrm{~N}_{5} \mathrm{O}_{4}\left(\mathrm{M}+\mathrm{H}^{+}\right)$, calcd 1286.5579, found 1286.5563; MALDI-MS found 1286.550 .

\section{5-(2-[N-(2,6-Diisopropylphenyl)-1,6-bis(4-tert-butylphenoxy)terrylene-3,4-} dicarboximid-9-yl]ethynyl)-15-(2-[ $N$-(2,6-diisopropylphenyl)-1,6-bis(4-tert-butylphenoxy) perylene-3,4-dicarboximid-9-yl]ethynyl)-10,20-diphenylporphyrin (PMI-P-TMI). Following a standard procedure, ${ }^{16,30,31}$ a Schlenk flask containing 7 (8.1 mg, $\left.6.3 \mu \mathrm{mol}\right)$, TMI-I (7.1 mg, 6.9 $\mu \mathrm{mol})$ and tri $(o$-tolyl)phosphine $(2.2 \mathrm{mg}, 7.2 \mu \mathrm{mol})$ was flushed with argon, treated with degassed toluene/TEA $(2.0 \mathrm{~mL}, 5: 1)$ and subjected to three freeze-pump-thaw cycles, then treated with tris(dibenzylideneacetone) dipalladium(0) $(2.2 \mathrm{mg}, 2.4 \mu \mathrm{mol})$ and subjected to two additional 
freeze-pump-thaw cycles. The resulting mixture was heated to $60{ }^{\circ} \mathrm{C}$ for $3 \mathrm{~h}$. The crude mixture was purified following the general procedure [silica (hexanes $/ \mathrm{CH}_{2} \mathrm{Cl}_{2}$ (2:1 to 1:1)); SEC (toluene); and silica (hexanes to hexanes/ $\left.\left.\mathrm{CH}_{2} \mathrm{Cl}_{2}(1: 1)\right)\right]$ to afford a dark brown solid (2.9 $\mathrm{mg} 21 \%$ ): ${ }^{1} \mathrm{H} \mathrm{NMR}$ $\left(600 \mathrm{MHz}, \mathrm{CDCl}_{3}\right) \delta-1.86(\mathrm{~s}, 2 \mathrm{H}), 1.17(\mathrm{~d}, J=7.0,24 \mathrm{H}), 1.30(\mathrm{~s}, 9 \mathrm{H}), 1.32(\mathrm{~s}, 9 \mathrm{H}), 1.35(\mathrm{~s}, 9 \mathrm{H})$, $1.35(\mathrm{~s}, 9 \mathrm{H}), 2.73-2.80(\mathrm{~m}, 4 \mathrm{H}), 6.87-6.96(\mathrm{~m}, 4 \mathrm{H}), 7.07-7.13(\mathrm{~m}, 4 \mathrm{H}), 7.28-7.40(\mathrm{~m}, 8 \mathrm{H}), 7.40-$ $7.49(\mathrm{~m}, 6 \mathrm{H}), 7.66-7.70(\mathrm{~m}, 2 \mathrm{H}), 7.74-7.85(\mathrm{~m}, 6 \mathrm{H}), 8.02(\mathrm{~d}, J=7.5 \mathrm{~Hz}, 1 \mathrm{H}), 8.06$ (d, $J=8.5 \mathrm{~Hz}$, $1 \mathrm{H}), 8.12-8.16(\mathrm{~m}, 4 \mathrm{H}), 8.16-8.30(\mathrm{~m}, 6 \mathrm{H}), 8.36(\mathrm{~s}, 2 \mathrm{H}), 8.67(\mathrm{~d}, J=7.9 \mathrm{~Hz}, 1 \mathrm{H}), 8.80(\mathrm{~d}, J=4.5$ $\mathrm{Hz}, 2 \mathrm{H}), 8.82(\mathrm{~d}, J=4.5 \mathrm{~Hz}, 2 \mathrm{H}), 9.01-9.11(\mathrm{~m}, 3 \mathrm{H}), 9.36-9.42(\mathrm{~m}, 2 \mathrm{H}), 9.61(\mathrm{~d}, J=4.5 \mathrm{~Hz}, 2 \mathrm{H})$, $9.72(\mathrm{~d}, J=4.5 \mathrm{~Hz}, 2 \mathrm{H}) ; \lambda_{\mathrm{abs}}$ (toluene) $440,540,658,749 \mathrm{~nm} ; \lambda_{\mathrm{em}}\left(\lambda_{\mathrm{exc}}=540 \mathrm{~nm}\right) 777 \mathrm{~nm}$. HRMS (ESI) for $\mathrm{C}_{154} \mathrm{H}_{124} \mathrm{~N}_{6} \mathrm{O}_{8}\left(\mathrm{M}+2 \mathrm{H}^{+}\right)$, calcd 1094.4846, found 1094.6393; MALDI-MS found $2185.730\left(\mathrm{M}+\mathrm{H}^{+}\right)$.

\section{5-(4-tert-Butylphenoxy)-10-mesityl-15-(2-[ $N$-(2,6-diisopropylphenyl)-1,6-bis(4-tert- butylphenoxy)terrylene-3,4-dicarboximid-9-yl]ethynyl)-18,18-dimethylchlorin (C-TMI). Following a standard procedure, ${ }^{16,30,31}$ a Schlenk flask containing samples of C-Br (4.1 mg, 6.2 $\mu \mathrm{mol})$, TMI-e $(5.2 \mathrm{mg}, 5.4 \mu \mathrm{mol})$ and tri $(o$-tolyl $)$ phosphine $(2.1 \mathrm{mg}, 6.9 \mu \mathrm{mol})$ was flushed with argon, treated with degassed toluene/TEA $(2.0 \mathrm{~mL}, 5: 1)$ and subjected to three freeze-pump-thaw cycles, then treated with tris(dibenzylidene-acetone)dipalladium(0) $(2.1 \mathrm{mg}, 2.3 \mu \mathrm{mol})$ and subjected to two additional freeze-pump-thaw cycles. The resulting mixture was heated to $60{ }^{\circ} \mathrm{C}$ for $3 \mathrm{~h}$. The crude mixture was purified following the general procedure [silica (hexanes $/ \mathrm{CH}_{2} \mathrm{Cl}_{2}$ (2:1 to 1:1)); SEC (toluene); and silica (hexanes to hexanes $/ \mathrm{CH}_{2} \mathrm{Cl}_{2}(1: 1)$ )] to afford a green solid (2.4 mg 32\%): ${ }^{1} \mathrm{H}$ NMR (600 MHz, $\left.\mathrm{CDCl}_{3}\right) \delta-0.97(\mathrm{~s}, 1 \mathrm{H}),-0.86(\mathrm{~s}, 1 \mathrm{H}), 1.17(\mathrm{~d}, J=6.7 \mathrm{~Hz}$, $12 \mathrm{H}), 1.33(\mathrm{~s}, 9 \mathrm{H}), 1.34(\mathrm{~s}, 9 \mathrm{H}), 1.91(\mathrm{~s}, 3 \mathrm{H}), 2.06(\mathrm{~s}, 3 \mathrm{H}), 2.61(\mathrm{~s}, 6 \mathrm{H}), 2.66(\mathrm{~s}, 6 \mathrm{H}), 2.76$ (pent, $J$ $=6.7 \mathrm{~Hz}, 2 \mathrm{H}), 4.81(\mathrm{~s}, 2 \mathrm{H}), 7.04-7.12(\mathrm{~m}, 4 \mathrm{H}), 7.25(\mathrm{~s}, 2 \mathrm{H}), 7.32(\mathrm{~d}, J=7.9 \mathrm{~Hz}, 2 \mathrm{H}), 7.37-7.44$ $(\mathrm{m}, 4 \mathrm{H}), 7.46(\mathrm{t}, J=7.9 \mathrm{~Hz}, 1 \mathrm{H}), 7.51(\mathrm{~d}, J=7.6 \mathrm{~Hz}, 2 \mathrm{H}), 7.82(\mathrm{t}, J=8.0 \mathrm{~Hz}, 1 \mathrm{H}), 7.99(\mathrm{~d}, J=7.6$ $\mathrm{Hz}, 2 \mathrm{H}), 8.12(\mathrm{~d}, J=7.7 \mathrm{~Hz}, 1 \mathrm{H}), 8.26(\mathrm{~d}, J=4.4 \mathrm{~Hz}, 1 \mathrm{H}), 8.26-8.32(\mathrm{~m}, 2 \mathrm{H}), 8.32-8.45(\mathrm{~m}, 5 \mathrm{H})$, $8.62(\mathrm{~d}, J=4.7 \mathrm{~Hz}, 1 \mathrm{H}), 8.65(\mathrm{~s}, 2 \mathrm{H}), 8.70(\mathrm{~s}, 1 \mathrm{H}), 8.84(\mathrm{~d}, J=8.0 \mathrm{~Hz}, 1 \mathrm{H}), 9.24-9.37(\mathrm{~m}, 3 \mathrm{H})$; $\lambda_{\text {abs }}$ (toluene) $412,672,705 \mathrm{~nm} ; \lambda_{\text {em }}\left(\lambda_{\text {exc }}=672 \mathrm{~nm}\right) 750 \mathrm{~nm}$. HRMS (ESI) for $\mathrm{C}_{104} \mathrm{H}_{89} \mathrm{~N}_{5} \mathrm{O}_{4}$ $\left(\mathrm{M}+2 \mathrm{H}^{+}\right)$, calcd 736.8530, found 736.8515; MALDI-MS found 1471.426 $\left(\mathrm{M}+\mathrm{H}^{+}\right)$.}

\section{5-(2-[N-(2,6-Diisopropylphenyl)-1,6-bis(4-tert-butylphenoxy)terrylene-3,4-} dicarboximid-9-yl]ethynyl)-15-methoxy-8,8,18,18-tetramethyl-2,12-bis(4-methylphenyl) bacteriochlorin (B-TMI). Following a standard procedure, ${ }^{16,30,31}$ a Schlenk flask containing BBr $(4.1 \mathrm{mg}, 6.5 \mu \mathrm{mol})$, TMI-e $(5.1 \mathrm{mg}, 5.5 \mu \mathrm{mol})$ and tri $(o$-tolyl $)$ phosphine $(2.2 \mathrm{mg}, 7.2 \mu \mathrm{mol})$ was flushed with argon, treated with degassed toluene/TEA $(2.0 \mathrm{~mL}, 5: 1)$ and subjected to three freeze-pump-thaw cycles, then treated with tris(dibenzylidene-acetone)dipalladium(0) (2.2 mg, $2.4 \mu \mathrm{mol})$ and subjected to two additional freeze-pump-thaw cycles. The resulting mixture was heated to $60{ }^{\circ} \mathrm{C}$ for $3 \mathrm{~h}$. The crude mixture was purified following the general procedure [silica (hexanes/ $\mathrm{CH}_{2} \mathrm{Cl}_{2}$ (2:1 to 1:1)), SEC (toluene), silica (hexanes to hexanes $/ \mathrm{CH}_{2} \mathrm{Cl}_{2}(1: 1)$ )] to afford a green solid $(2.2 \mathrm{mg} 29 \%):{ }^{1} \mathrm{H}$ NMR $\left(600 \mathrm{MHz}, \mathrm{CDCl}_{3}\right) \delta-1.53(\mathrm{~s}, 1 \mathrm{H}),-1.25(\mathrm{~s}, 1 \mathrm{H}), 1.19(\mathrm{~d}, J$ $=6.8 \mathrm{~Hz}, 12 \mathrm{H}), 1.33(\mathrm{~s}, 9 \mathrm{H}), 1.34(\mathrm{~s}, 9 \mathrm{H}), 1.93(\mathrm{~s}, 6 \mathrm{H}), 1.94(\mathrm{~s}, 6 \mathrm{H}), 2.61(\mathrm{~s}, 3 \mathrm{H}), 2.64(\mathrm{~s}, 3 \mathrm{H})$, 2.77 (pent, $J=6.8 \mathrm{~Hz}, 2 \mathrm{H}), 4.34(\mathrm{~s}, 2 \mathrm{H}), 4.47(\mathrm{~s}, 3 \mathrm{H}), 4.68(\mathrm{~s}, 2 \mathrm{H}), 7.00-7.09(\mathrm{~m}, 4 \mathrm{H}), 7.32(\mathrm{~d}, J$ $=8.0 \mathrm{~Hz}, 2 \mathrm{H}), 7.37-7.48(\mathrm{~m}, 5 \mathrm{H}), 7.58(\mathrm{~d}, J=7.5 \mathrm{~Hz}, 2 \mathrm{H}), 7.62(\mathrm{~d}, J=7.5 \mathrm{~Hz}, 2 \mathrm{H}), 7.80(\mathrm{t}, J=$ $7.7 \mathrm{~Hz}, 1 \mathrm{H}), 8.09$ (d, $J=7.5 \mathrm{~Hz}, 3 \mathrm{H}), 8.17$ (d, $J=7.5 \mathrm{~Hz}, 2 \mathrm{H}), 8.19-8.27$ (m, 2H), 8.29-8.38 (m, 4H), $8.77(\mathrm{~s}, 1 \mathrm{H}), 8.78-8.88(\mathrm{~m}, 3 \mathrm{H}), 9.20(\mathrm{~s}, 1 \mathrm{H}), 9.21-9.29(\mathrm{~m}, 2 \mathrm{H}) ; \lambda_{\mathrm{abs}}$ (toluene) 383, 556, 685, $774 \mathrm{~nm} ; \lambda_{\text {em }}\left(\lambda_{\text {exc }}=556 \mathrm{~nm}\right) 785 \mathrm{~nm}$. HRMS (ESI) for $\mathrm{C}_{105} \mathrm{H}_{93} \mathrm{~N}_{5} \mathrm{O}_{5}\left(\mathrm{M}^{2+}\right)$, calcd 751.8582 , found 751.8576; MALDI-MS found $1503.663\left(\mathrm{M}+\mathrm{H}^{+}\right)$. 
影

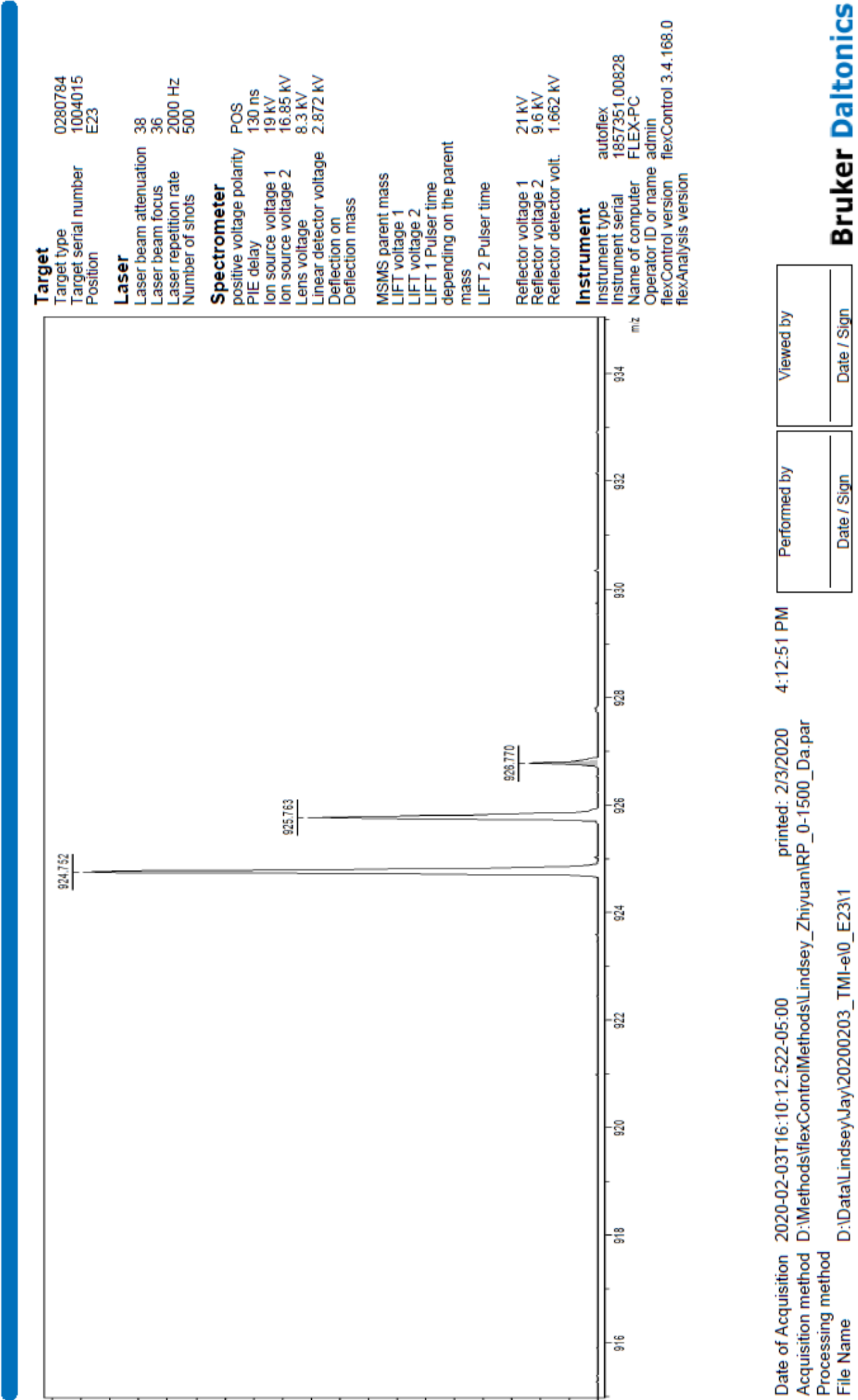

Figure S1. MALDI-MS data of compound TMI-e. 


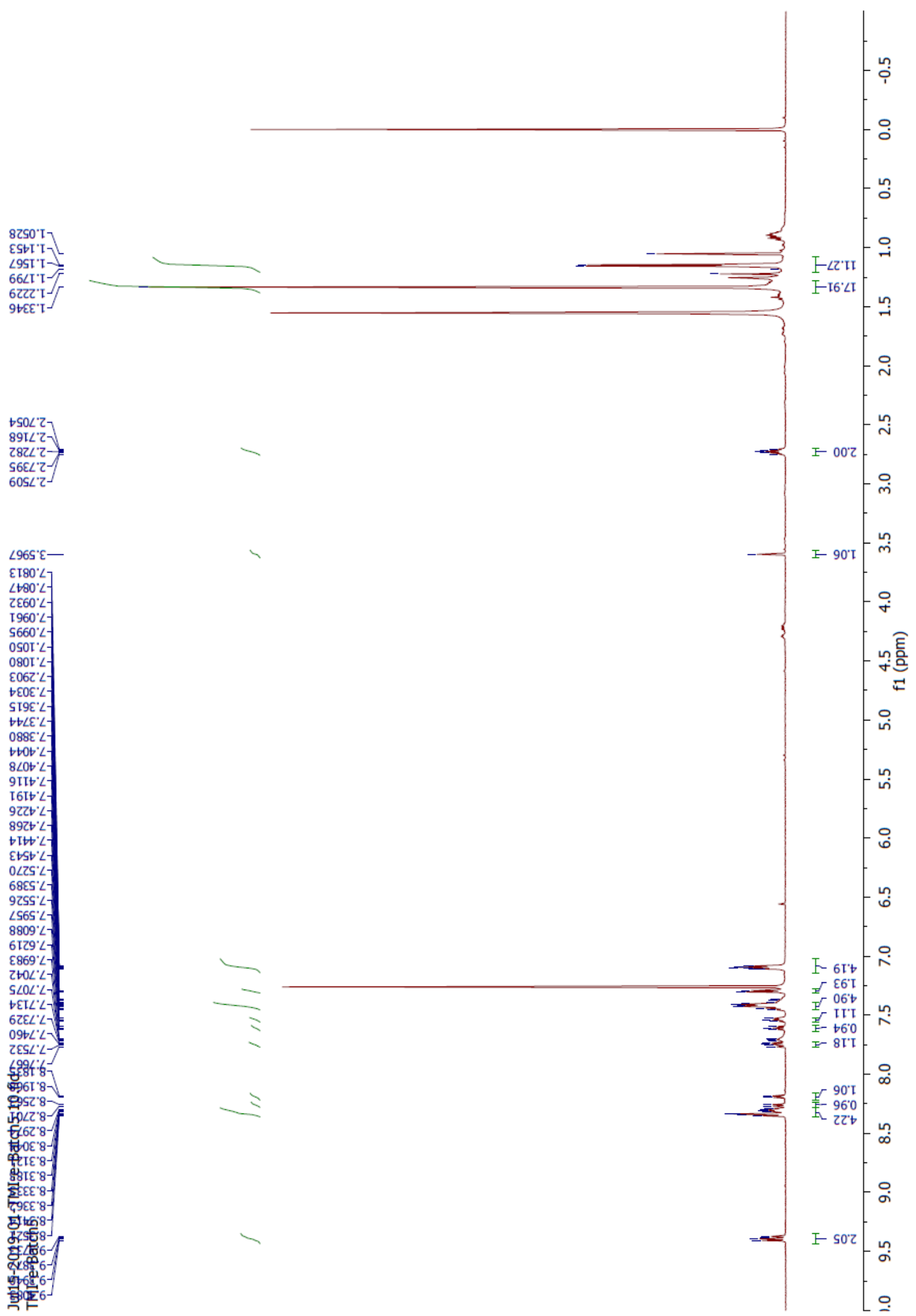

Figure S2. ${ }^{1} \mathrm{H}$ NMR spectrum of compound TMI-e. 
NL:

8.89E5

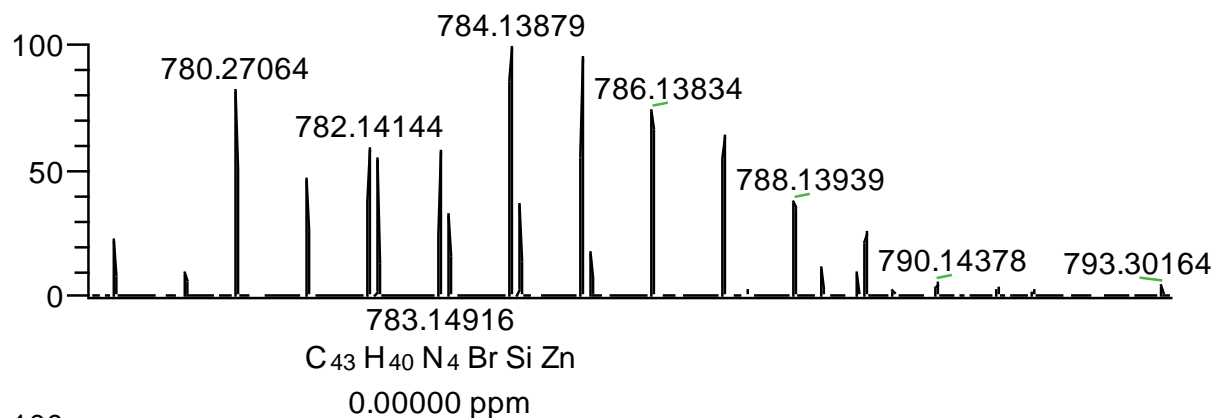

193269 Jay10 SYR\#20

0-335 RT: 0.89-1.49

AV: $136 \mathrm{~T}: \mathrm{FTMS}+\mathrm{p}$ ESI

Full ms

[200.0000-2500.0000]

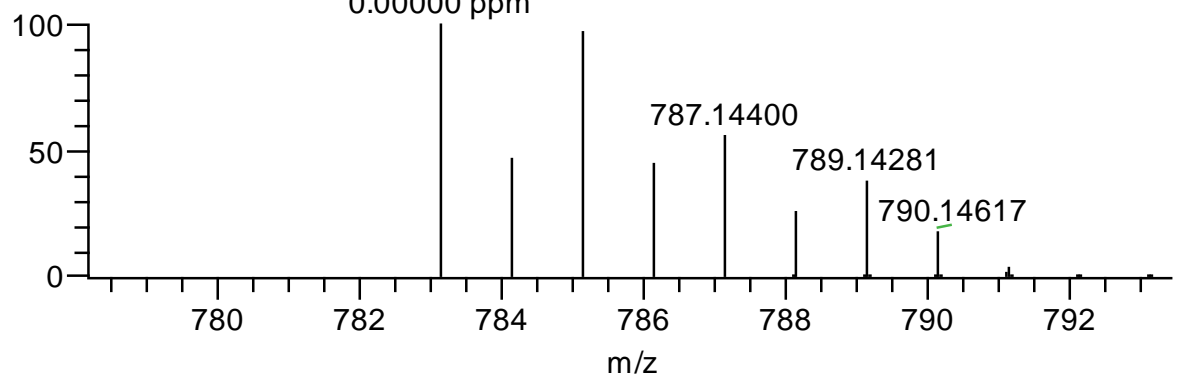

1.40E5

$\mathrm{C}_{43} \mathrm{H}_{39} \mathrm{BrN}_{4} \mathrm{SiZn}+\mathrm{H}$ :

$\mathrm{C}_{43} \mathrm{H}_{40} \mathrm{Br}_{1} \mathrm{~N}_{4} \mathrm{Si}_{1} \mathrm{Zn}_{1}$ pa Chrg 1

Figure S3. HRMS (ESI) data of compound 4 (upper) and calculation (lower). 


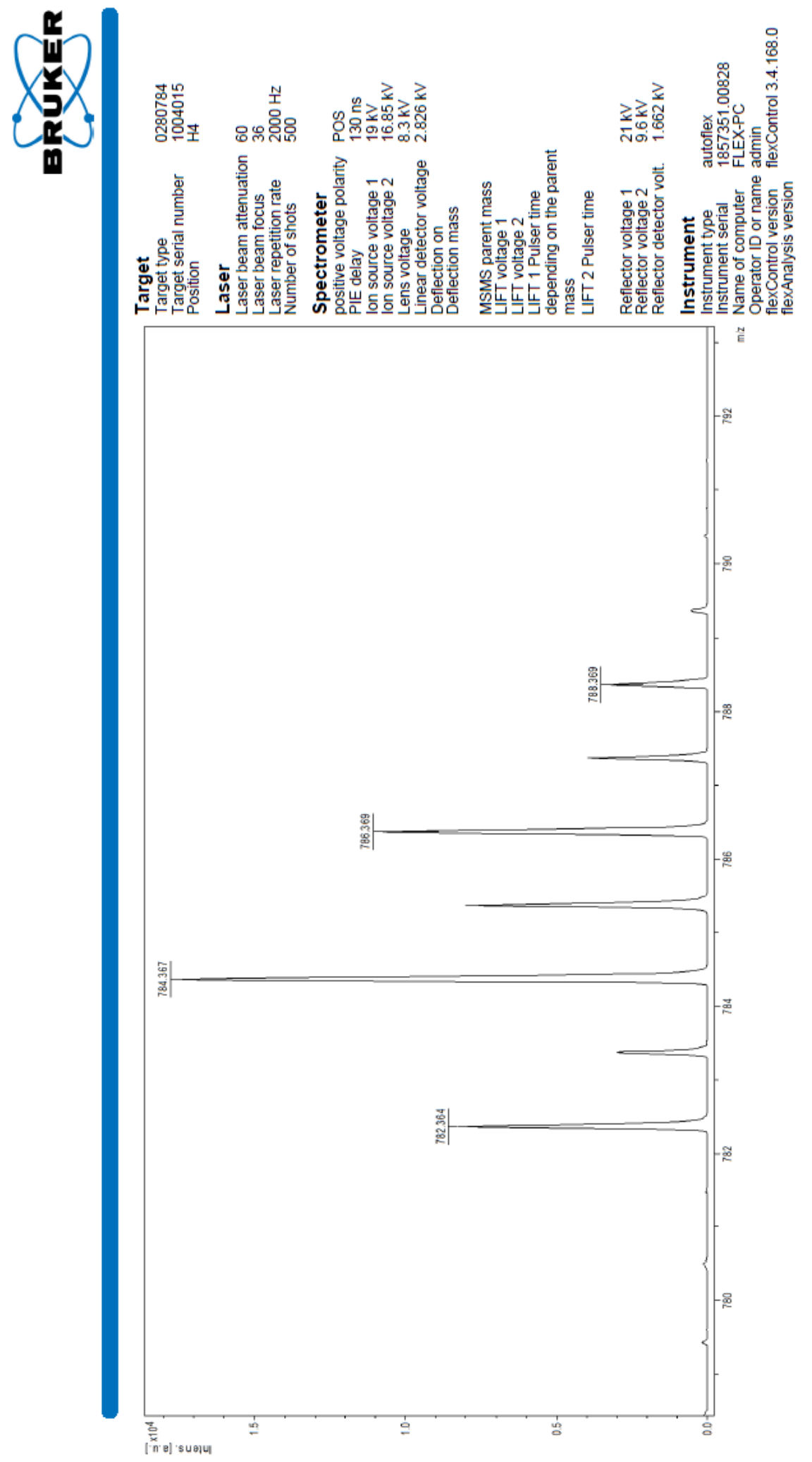

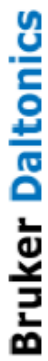
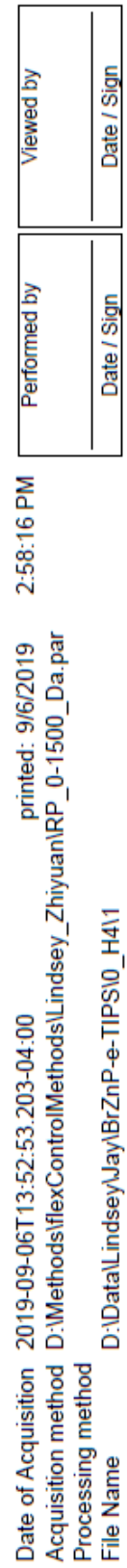

Figure S4. MALDI-MS data of compound 4. 


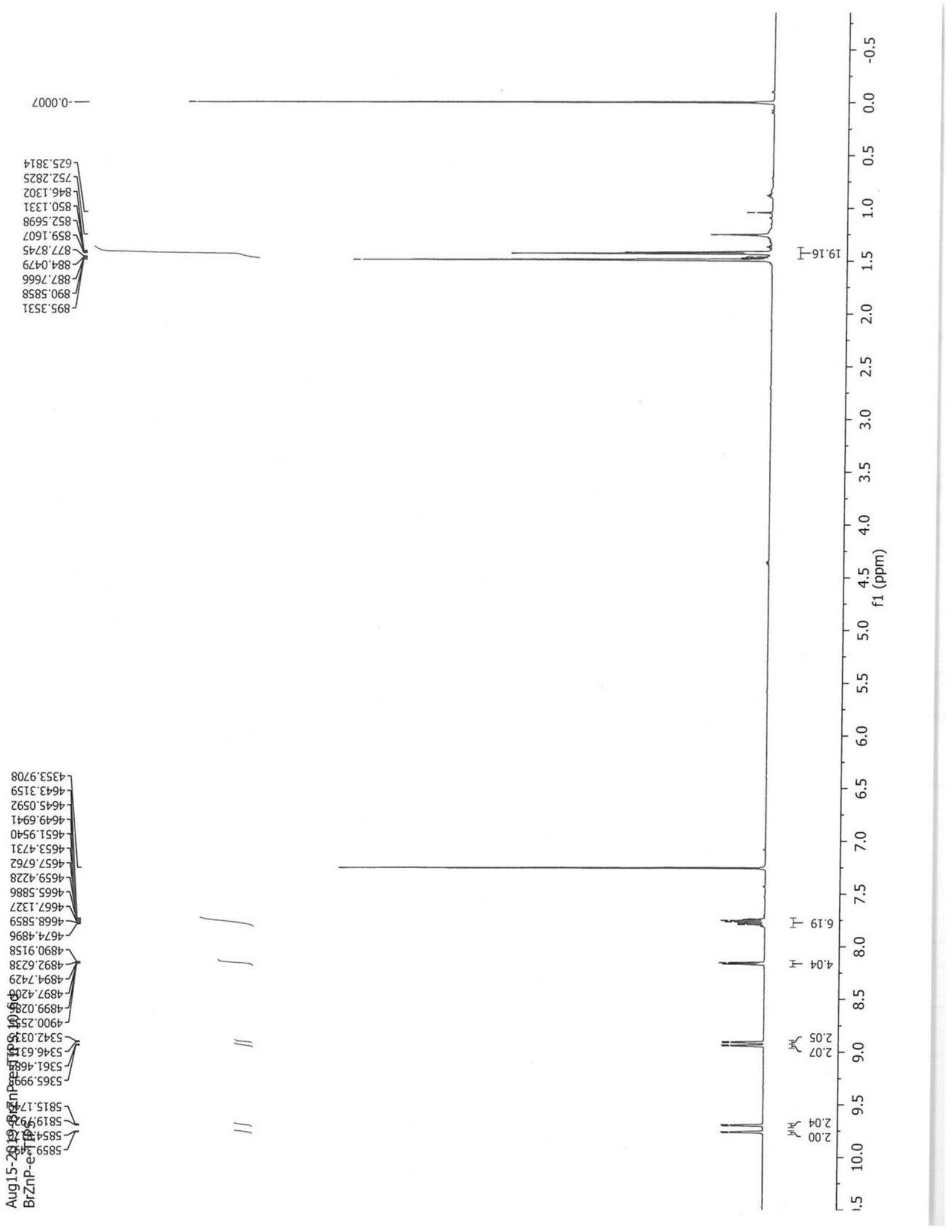

Figure S5. ${ }^{1} \mathrm{H}$ NMR spectrum of compound 4. 
NL:

$1.82 \mathrm{E} 7$

193272_Jay11_SYR\#11 -151 RT: $0.05-\overline{0} .67 \mathrm{AV}$ :

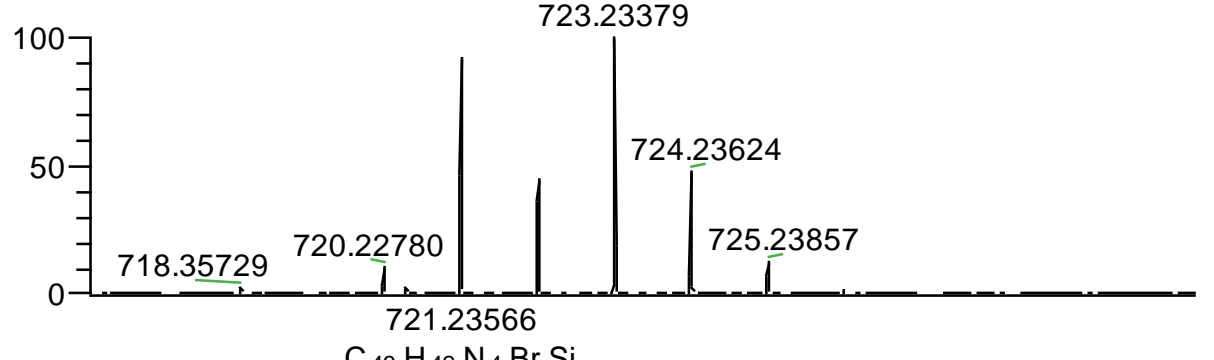
$141 \mathrm{~T}: \mathrm{FTMS}+\mathrm{p}$ ESI

Full ms

[200.0000-2500.0000]

NL:

2.89E5

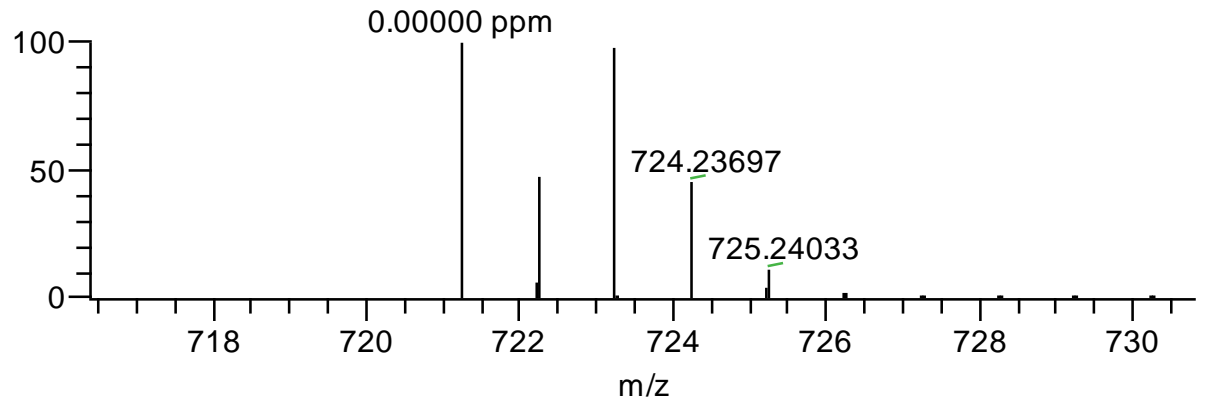

$\mathrm{C}_{43} \mathrm{H}_{41} \mathrm{BrN}_{4} \mathrm{Si}+\mathrm{H}$ :

$\mathrm{C}_{43} \mathrm{H}_{42} \mathrm{Br}_{1} \mathrm{~N}_{4} \mathrm{Si}_{1}$ pa Chrg 1

Figure S6. HRMS (ESI) data of compound 5 (upper) and calculation (lower). 


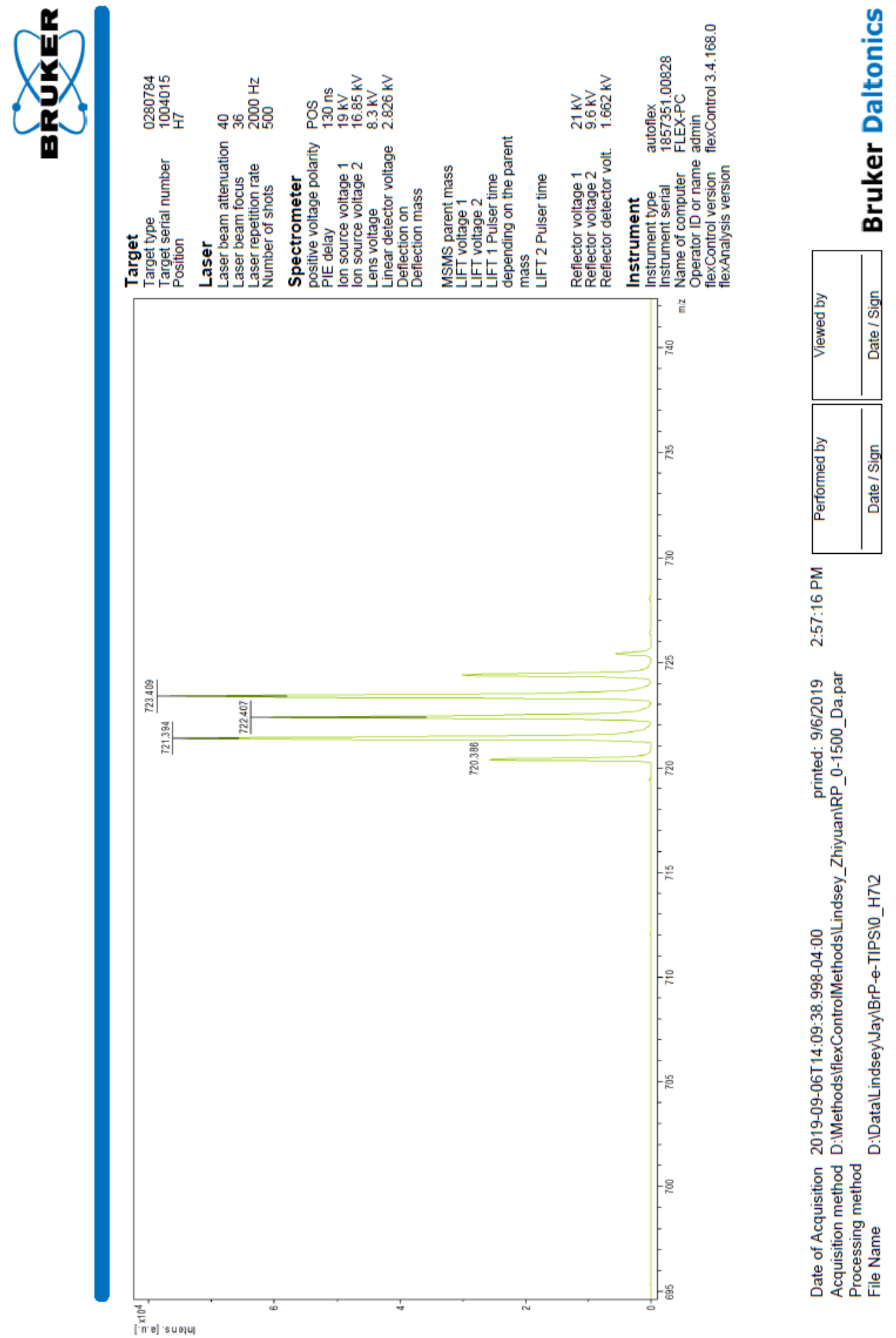

Figure S7. MALDI-MS data of compound 5. 


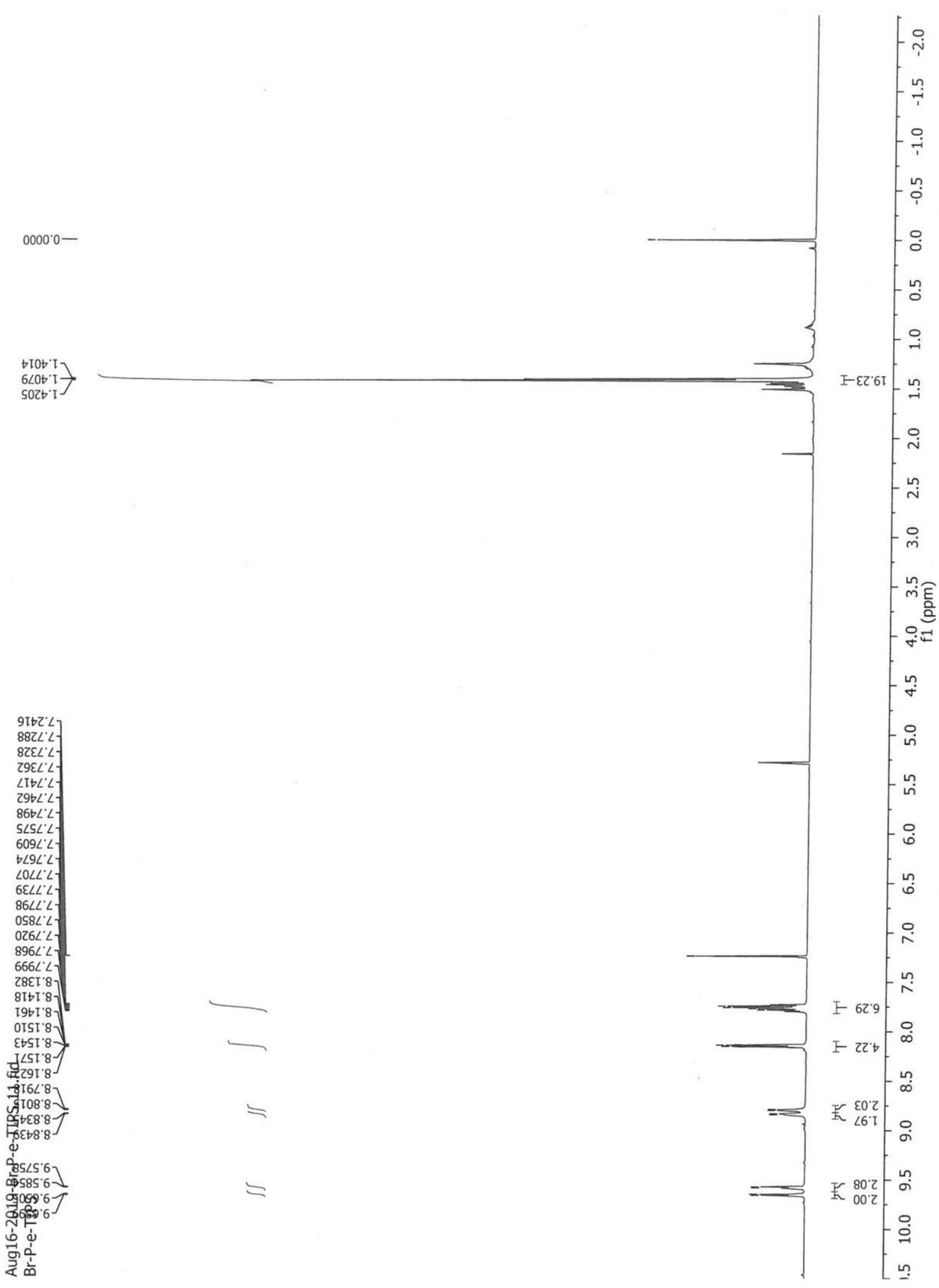

Figure S8. ${ }^{1} \mathrm{H}$ NMR spectrum of compound 5. 
$\mathrm{NL}$ :

2.07E6

193274 Jay12 SYR\#29

2-388 RT: 1.30-1.73

AV: 97 T: FTMS + p ESI

Full ms

[200.0000-2500.0000]
NL:

3.28E5

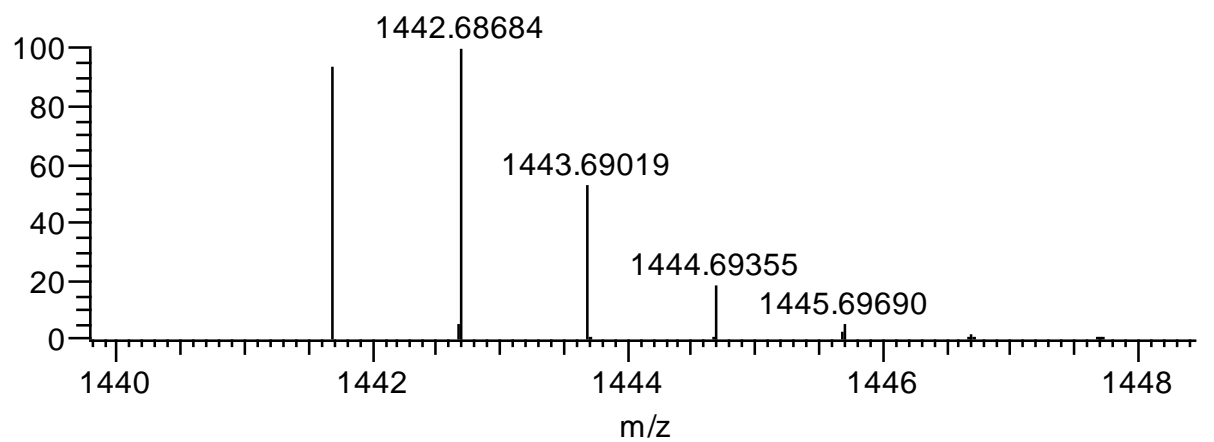

$\mathrm{C}_{99} \mathrm{H}_{91} \mathrm{~N}_{5} \mathrm{O}_{4} \mathrm{Si}:$

$\mathrm{C}_{99} \mathrm{H}_{91} \mathrm{~N}_{5} \mathrm{O}_{4} \mathrm{Si}_{1}$ pa Chrg 1

Figure S9. HRMS (ESI) data of compound 6 (upper) and calculation (lower). 

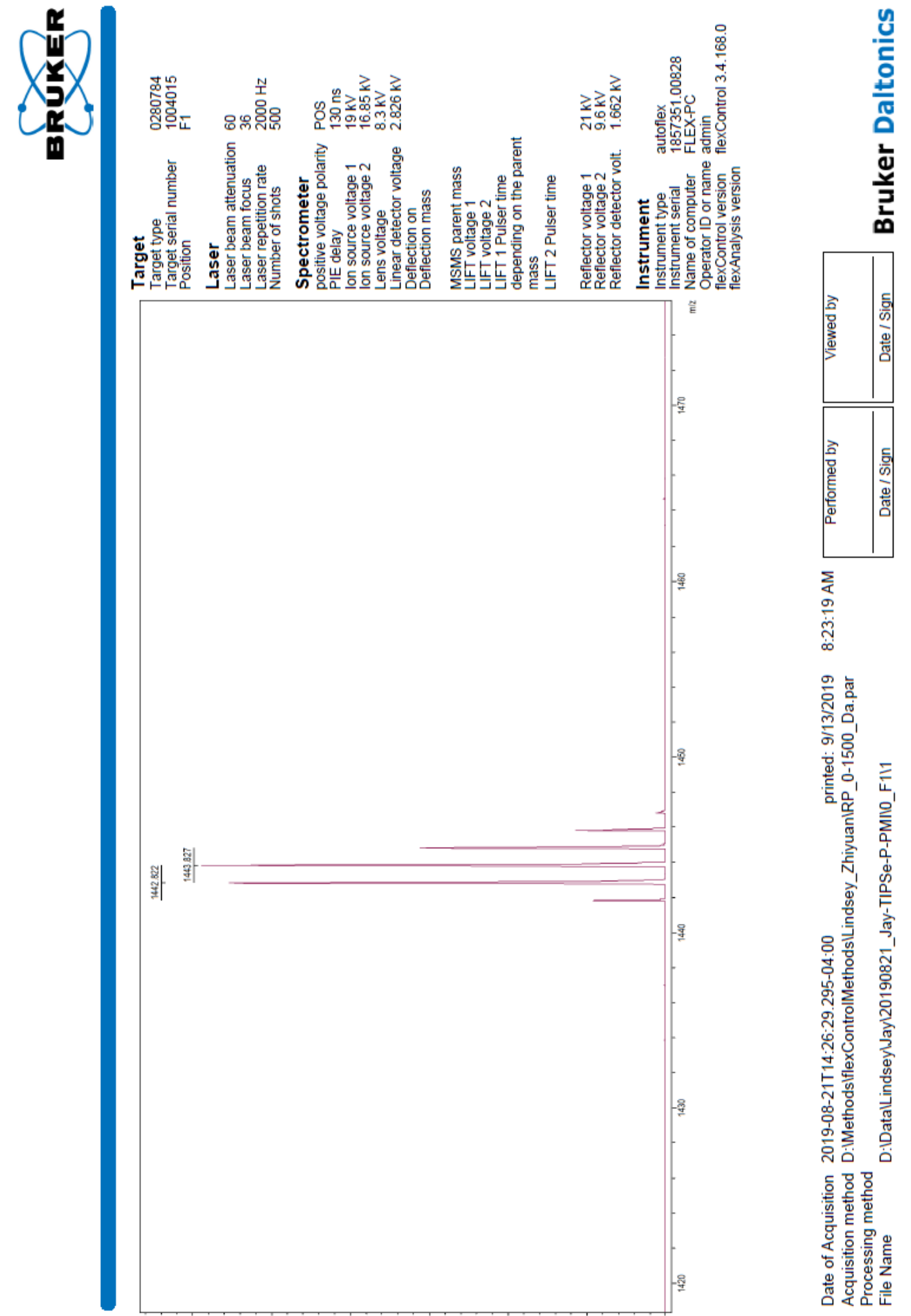

Figure S10. MALDI-MS data of compound 6. 


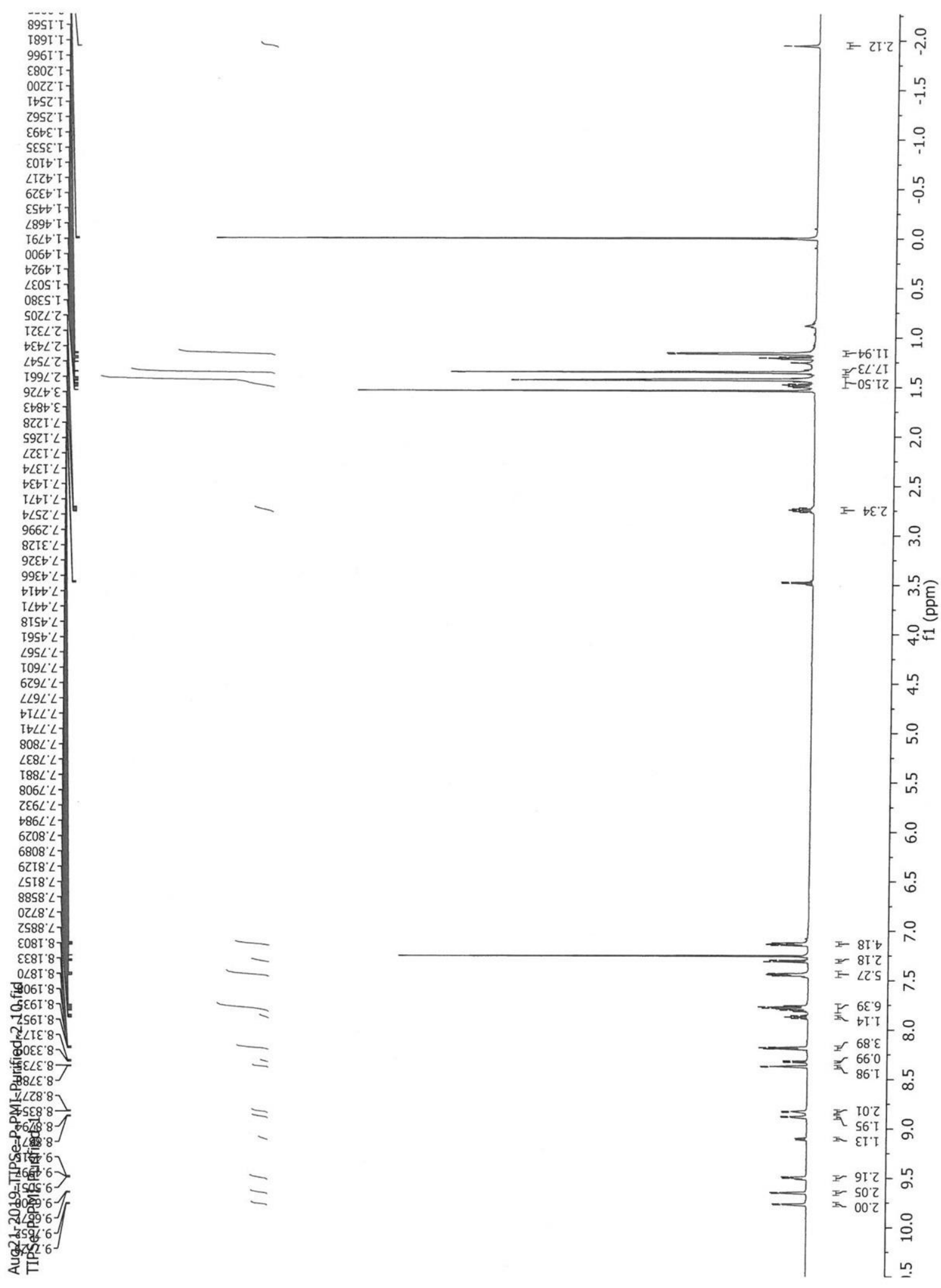

Figure S11. ${ }^{1} \mathrm{H}$ NMR spectrum of compound 6. 


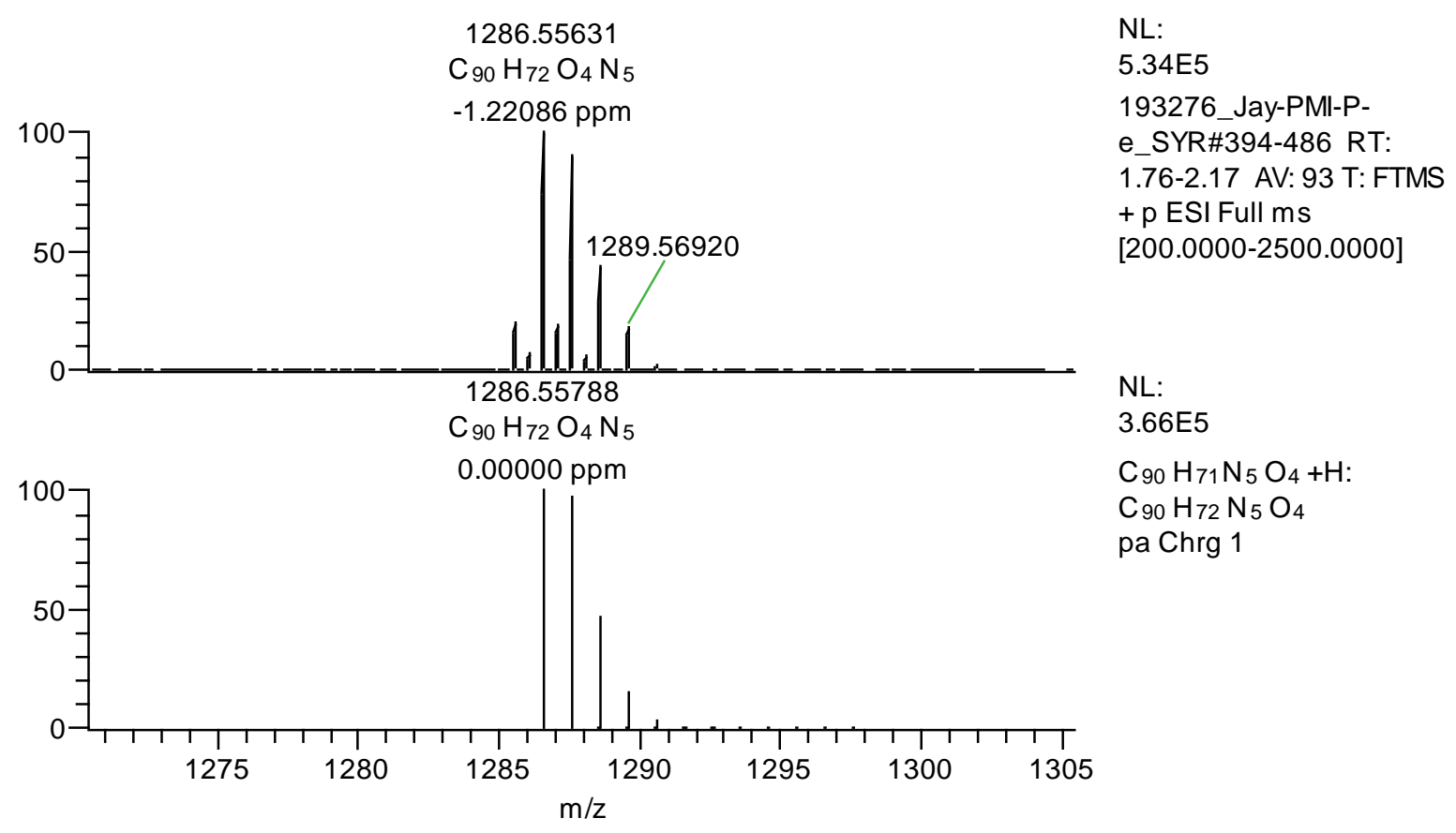

Figure S12. HRMS (ESI) data of compound 7 (upper) and calculation (lower). 


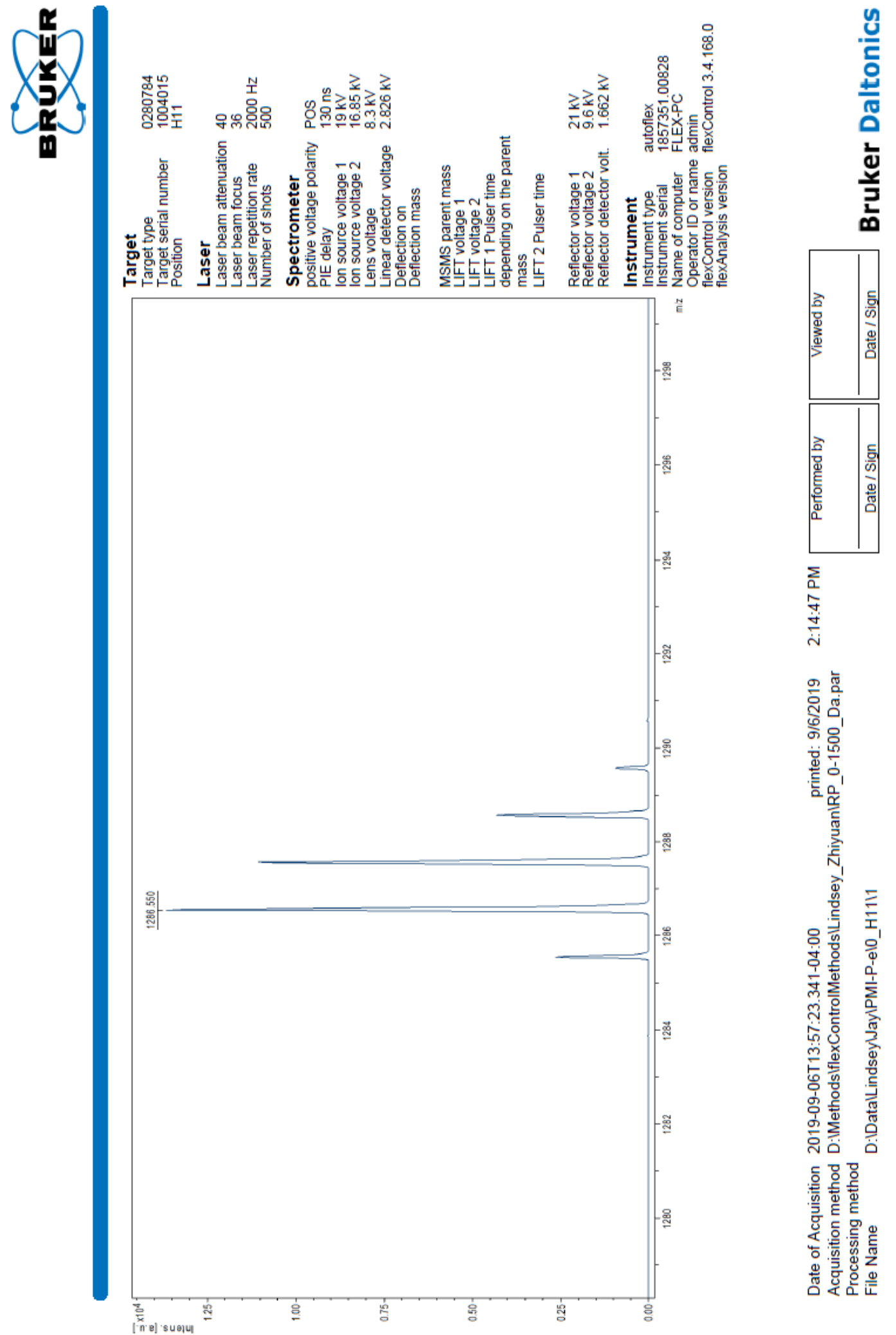

Figure S13. MALDI-MS data of compound 7. 


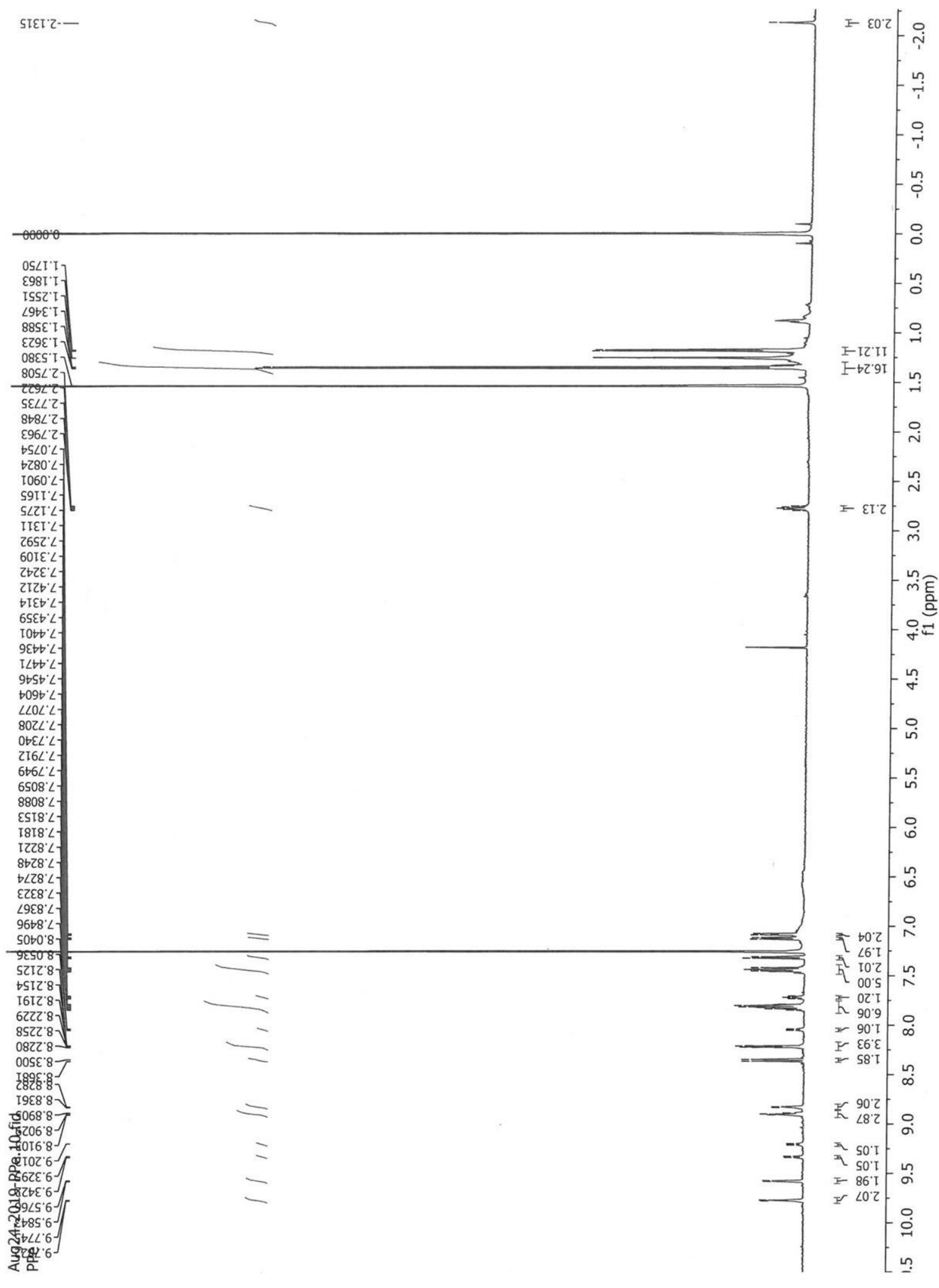

Figure S14. ${ }^{1} \mathrm{H}$ NMR spectrum of compound 7. 
$\mathrm{NL}$ :

$1.45 \mathrm{E} 4$

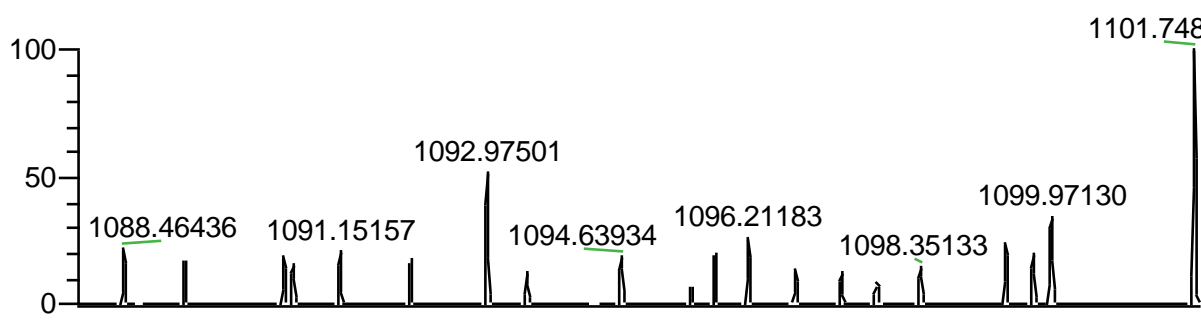

$\mathrm{NL}:$

3.00E5

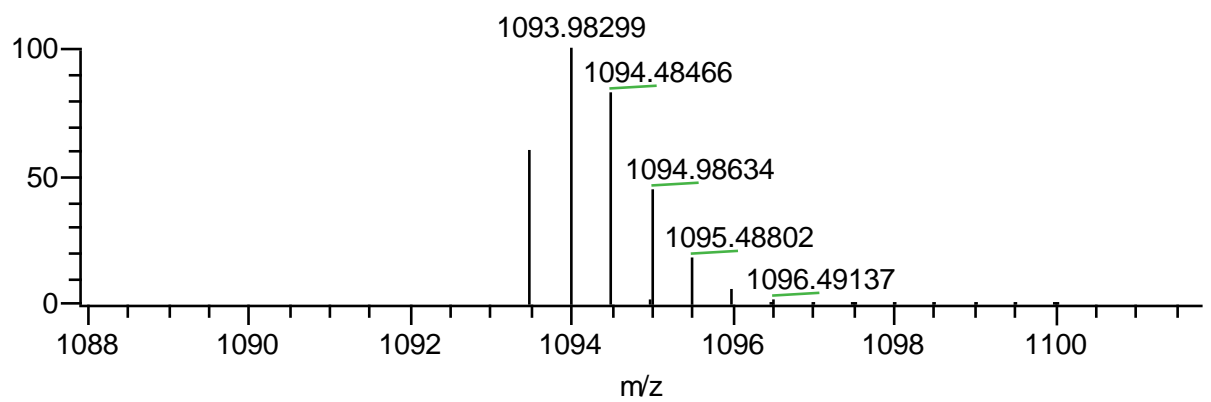

$\mathrm{C}_{154} \mathrm{H}_{124} \mathrm{~N}_{6} \mathrm{O}_{8}+\mathrm{H}$ : $\mathrm{C}_{154} \mathrm{H}_{126} \mathrm{~N}_{6} \mathrm{O}_{8}$ pa Chrg 2

Figure S15. HRMS (ESI) data of compound PMI-P-TMI (upper) and calculation (lower). 


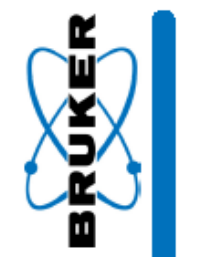

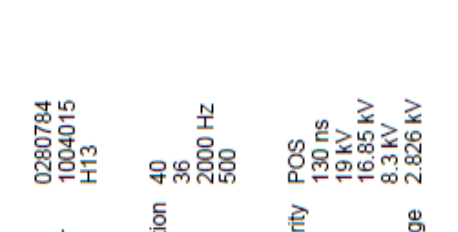

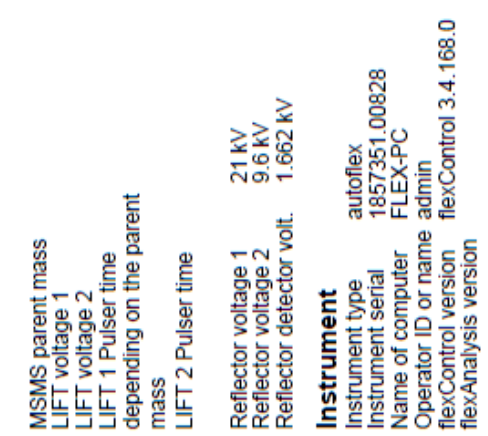

늘

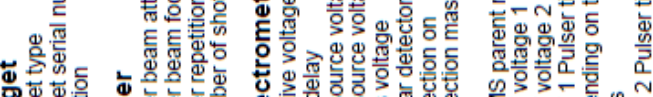

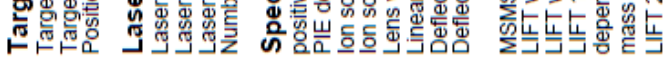
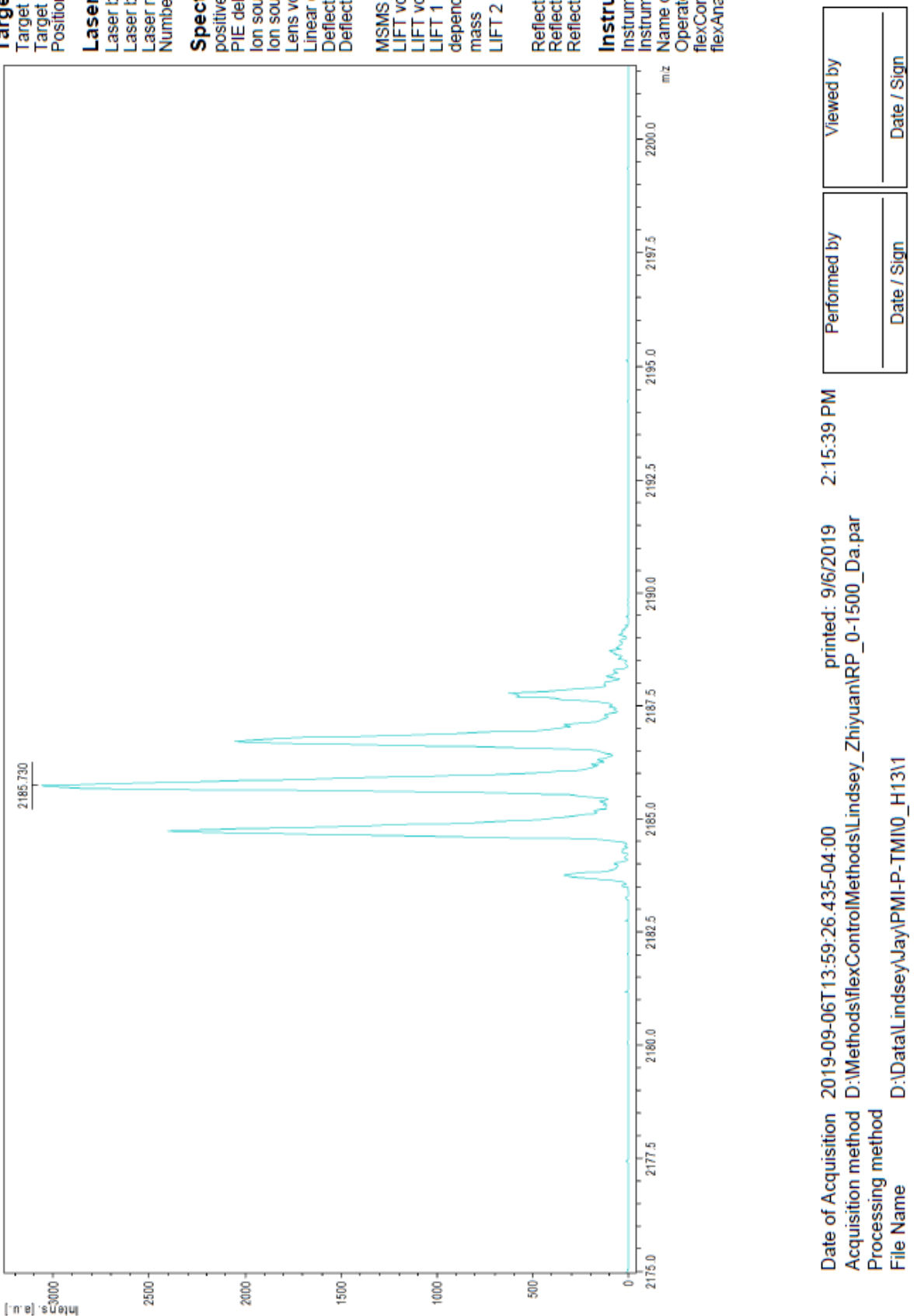

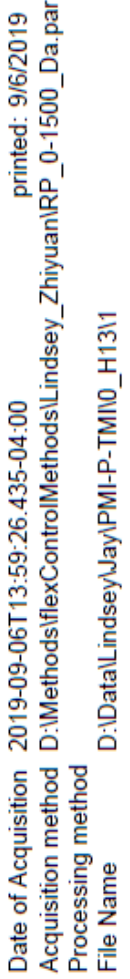

Figure S16. MALDI-MS data of compound PMI-P-TMI. 


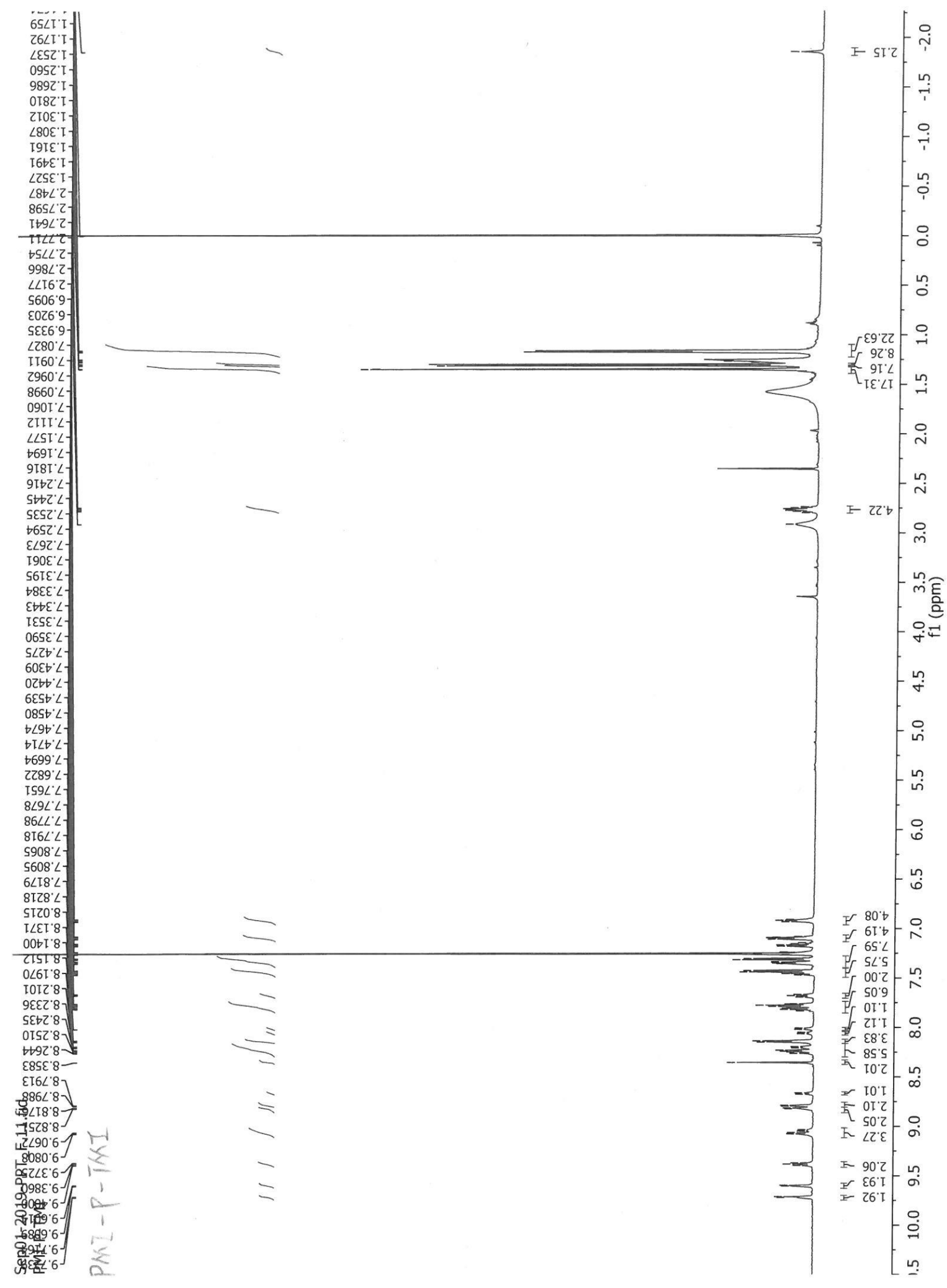

Figure S17. ${ }^{1} \mathrm{H}$ NMR spectrum of compound PMI-P-TMI. 
$\mathrm{NL}:$

1.69E6

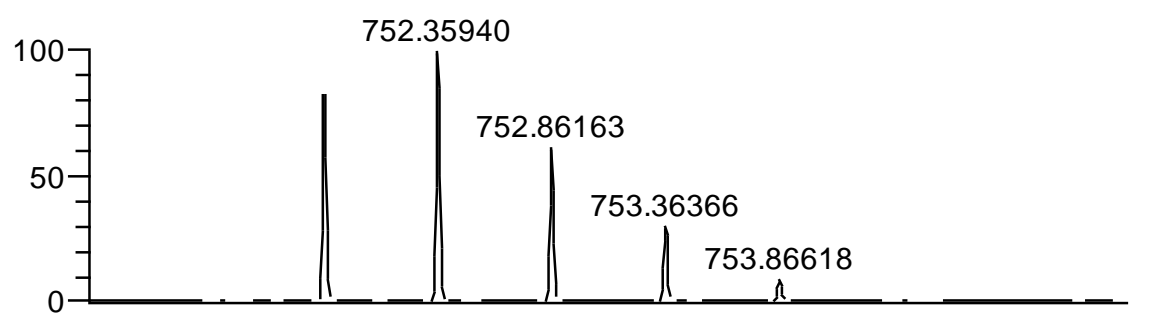

193280 Jay-BC-

TMI SYR\#364-496 RT:

1.62-2.21 AV: $133 \mathrm{~T}$ :

FTMS + p ESI Full ms

[200.0000-2500.0000]

NL:

3.52E5

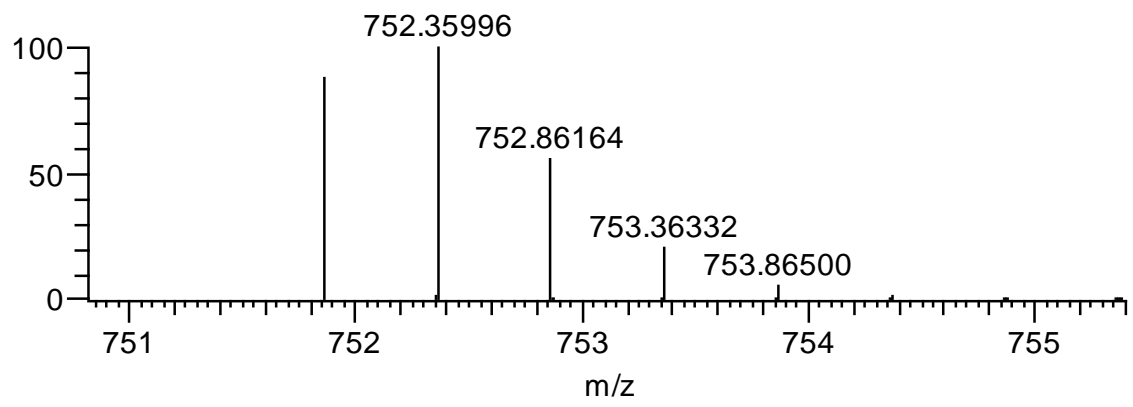

$\mathrm{C}_{105} \mathrm{H}_{93} \mathrm{~N}_{5} \mathrm{O}_{5}$ : $\mathrm{C}_{105} \mathrm{H}_{93} \mathrm{~N}_{5} \mathrm{O}_{5}$ pa Chrg 2

Figure S18. HRMS (ESI) data of compound B-TMI (upper) and calculation (lower). 

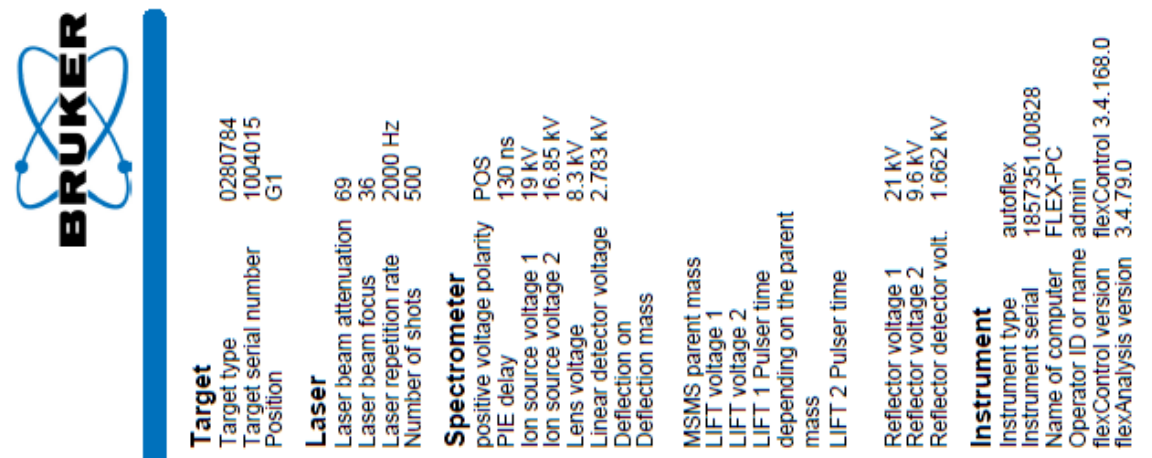

들
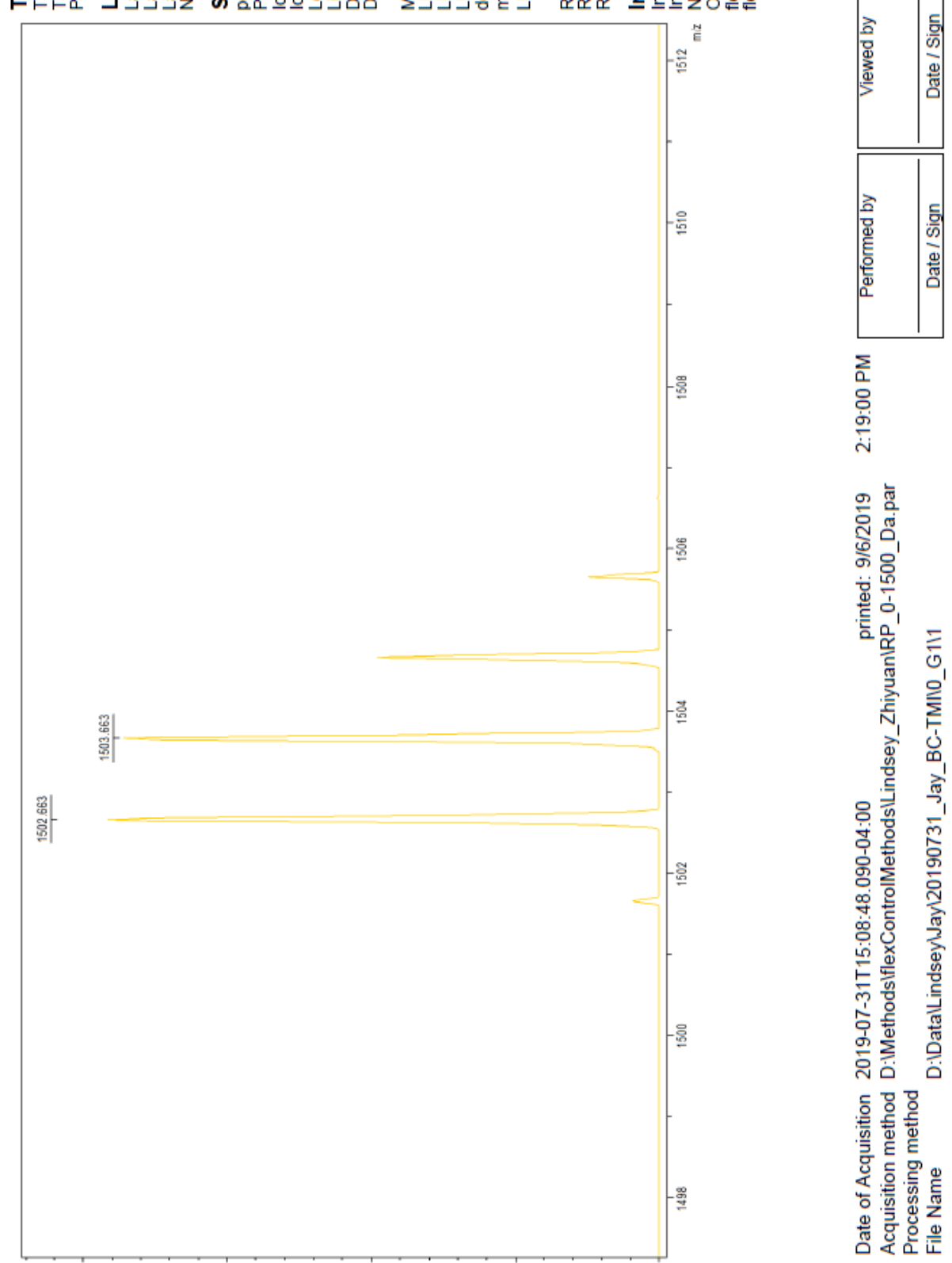

Figure S19. MALDI-MS data of compound B-TMI. 


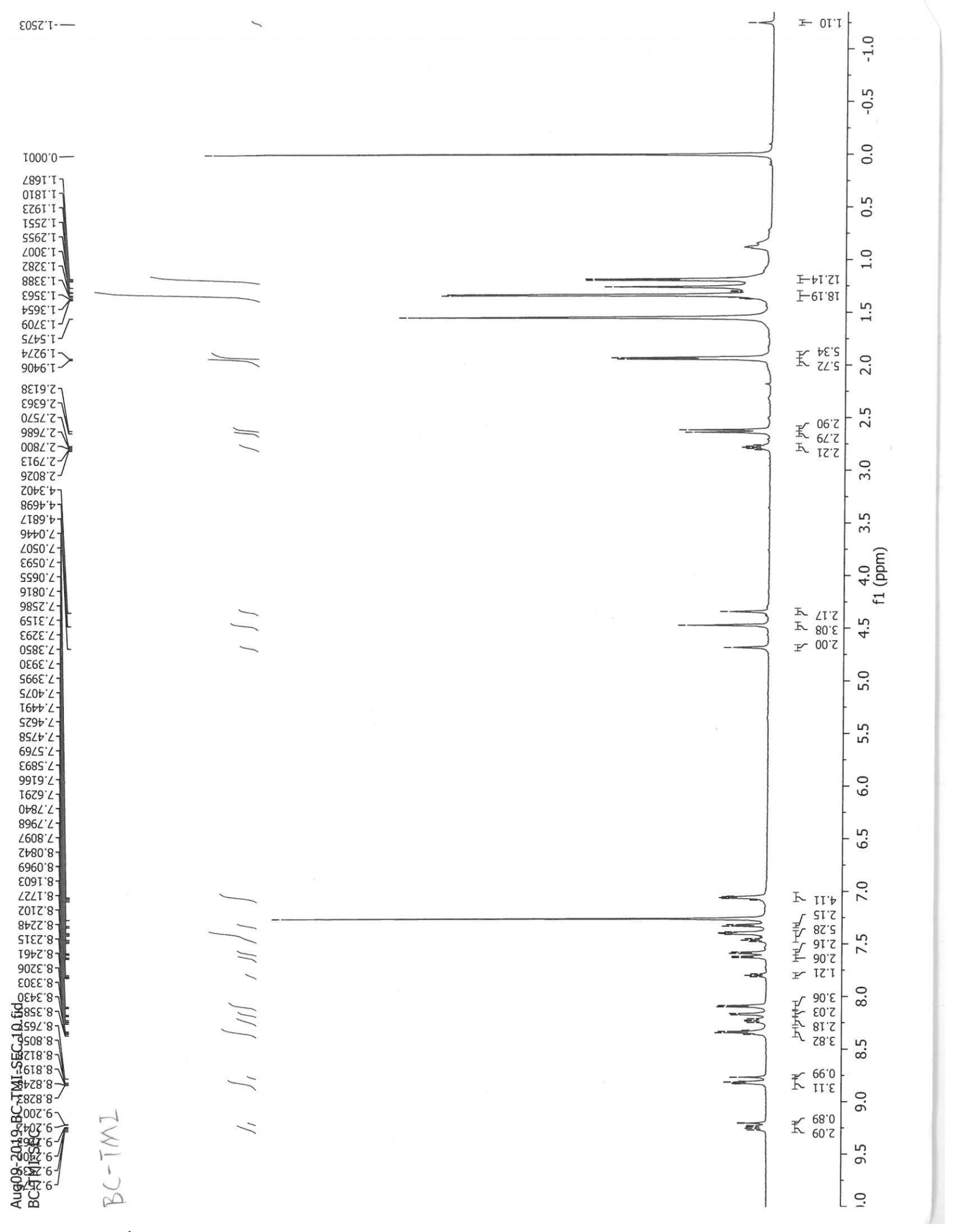

Figure S20. ${ }^{1} \mathrm{H}$ NMR spectrum of compound B-TMI. 


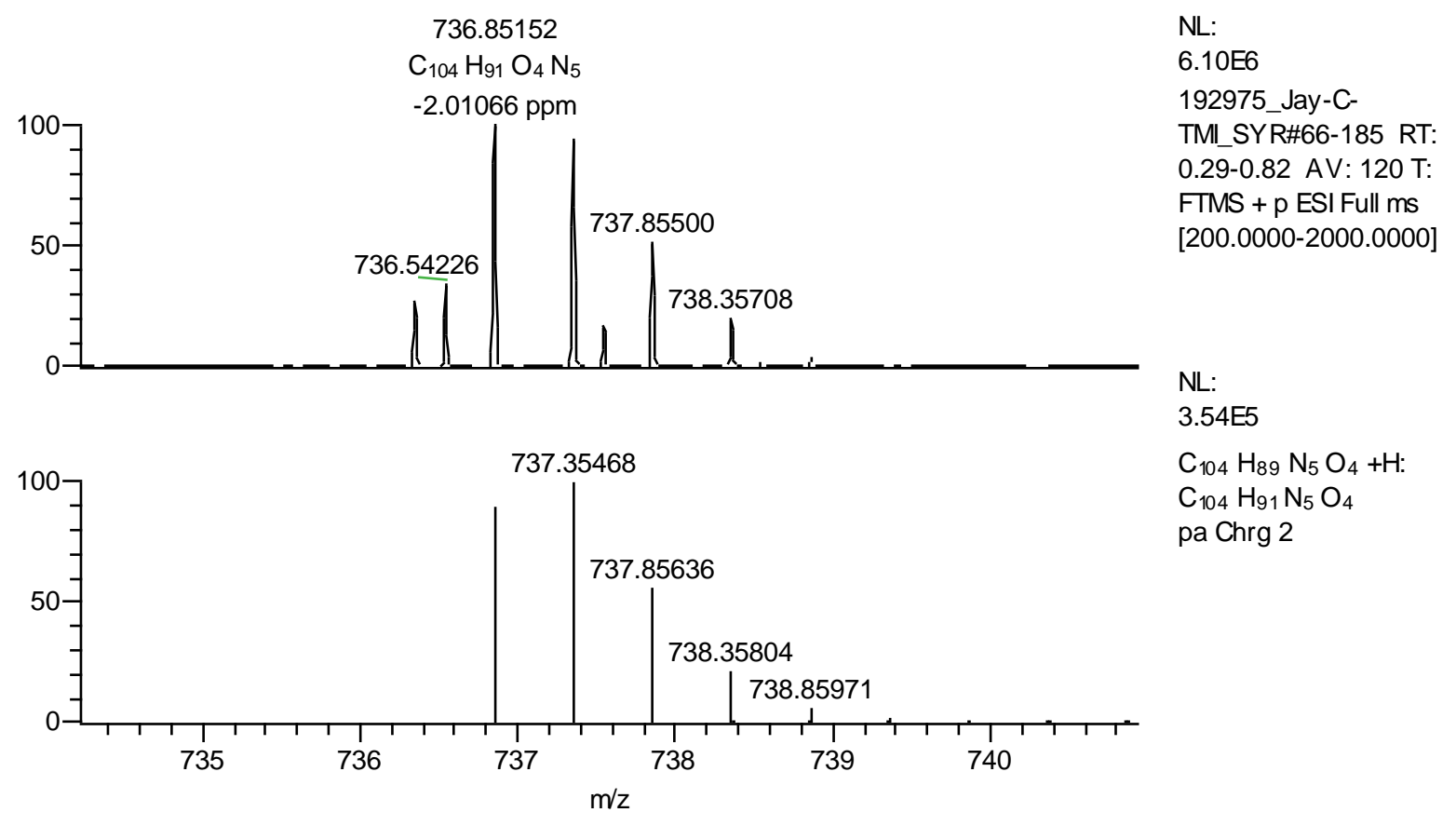

Figure S21. HRMS (ESI) data of compound C-TMI (upper) and calculation (lower). 


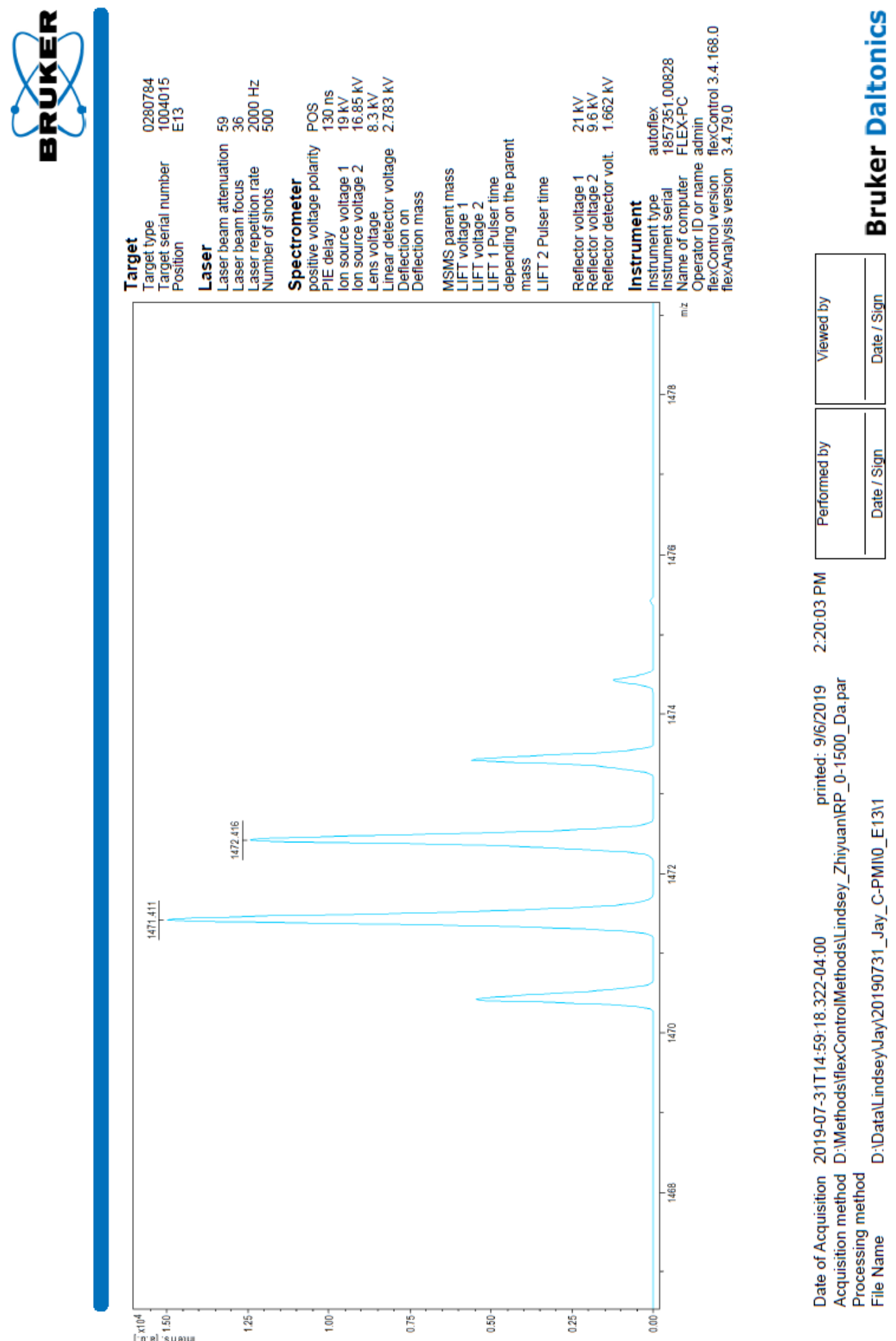

Figure S22. MALDI-MS data of compound C-TMI. 


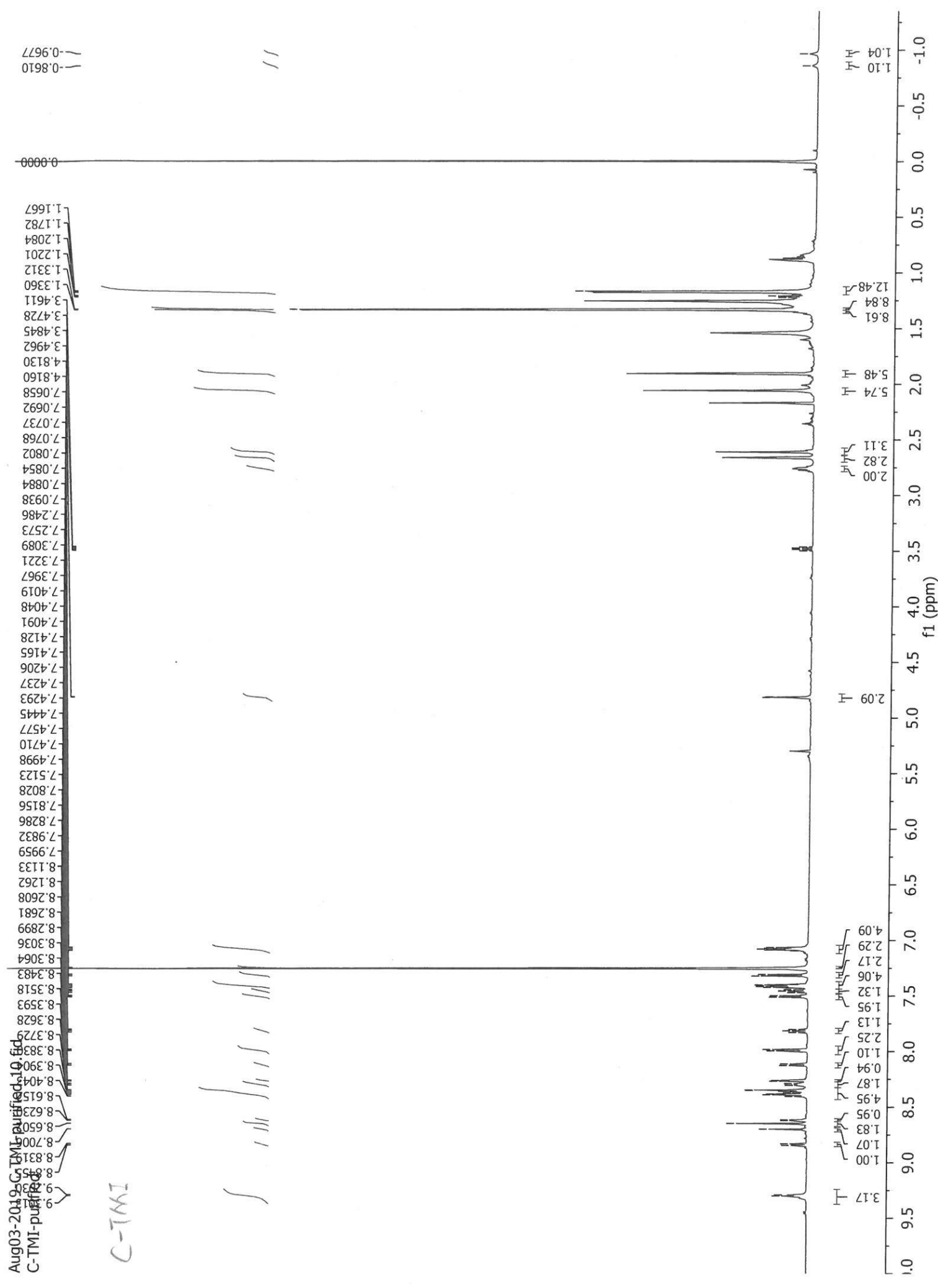

Figure S23. ${ }^{1} \mathrm{H}$ NMR spectrum of compound C-TMI. 


\section{Topic S1-2. Analytical SEC monitoring of the formation of PMI-P-TMI triad.}

The Sonogashira coupling reaction of compound 7 (= PMI-P-e) and TMI-I was monitored with analytical size exclusive chromatography (SEC), as has been done previously with other arrays $^{34}$ including panchromatic arrays. ${ }^{14,16}$ The starting materials analyzed by analytical SEC to determine the retention time (Figure S24, Panel A). After the three-hour reaction, an aliquot of the reaction mixture was analyzed to show the formation of a new leading peak (Panel B). The dominant peak was assigned to the TMI-I related species based on the retention time and absorption data. The retention time of the purified triad (Panel C), mass spectroscopy, NMR and absorption data revealed that the leading peak in panel B was the desired triad product.
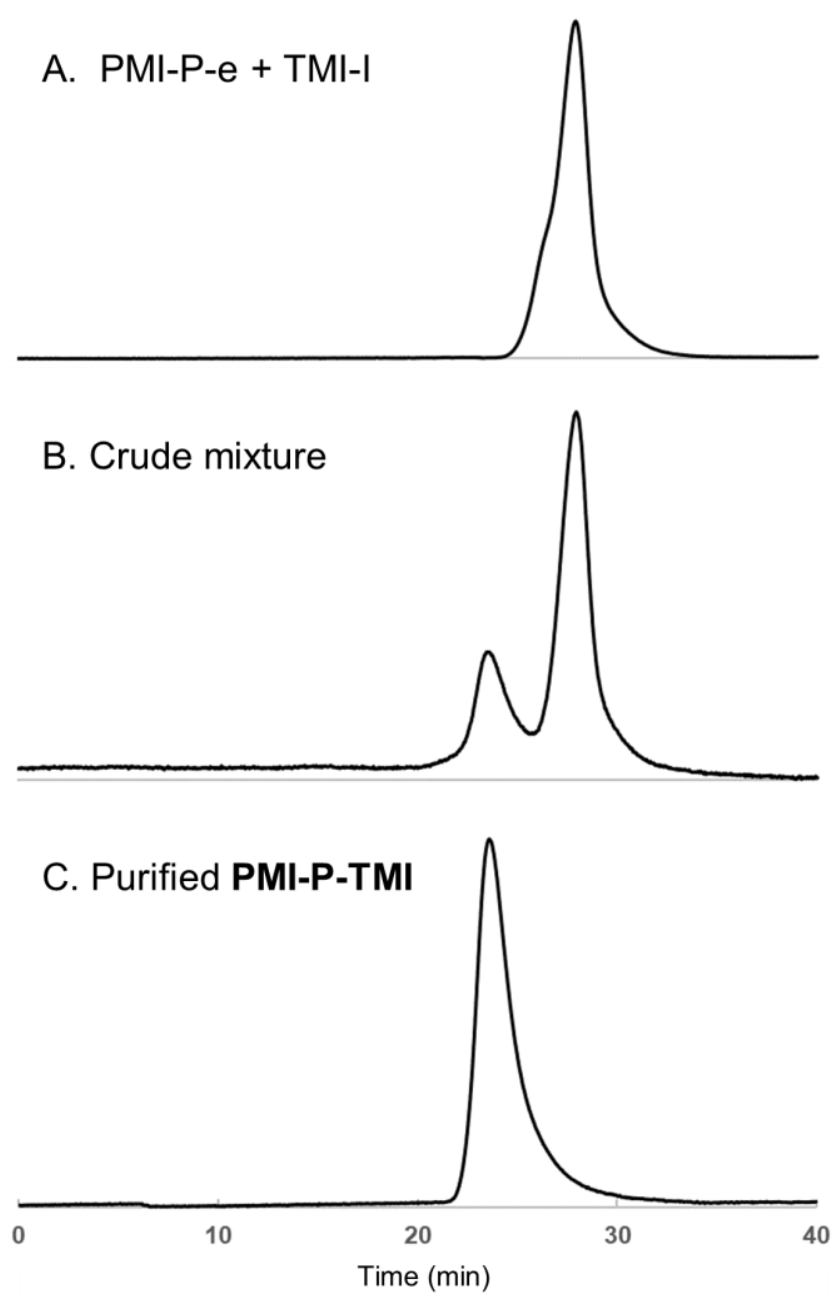

Figure S24. Analytical SEC traces $\left(\lambda_{\text {det }}=630 \mathrm{~nm}\right)$ for the synthesis of PMI-P-TMI triad. (A) Mixture of the starting materials. (B) Crude mixture after reaction for $3 \mathrm{~h}$. (C) Purified PMI-PTMI triad. 


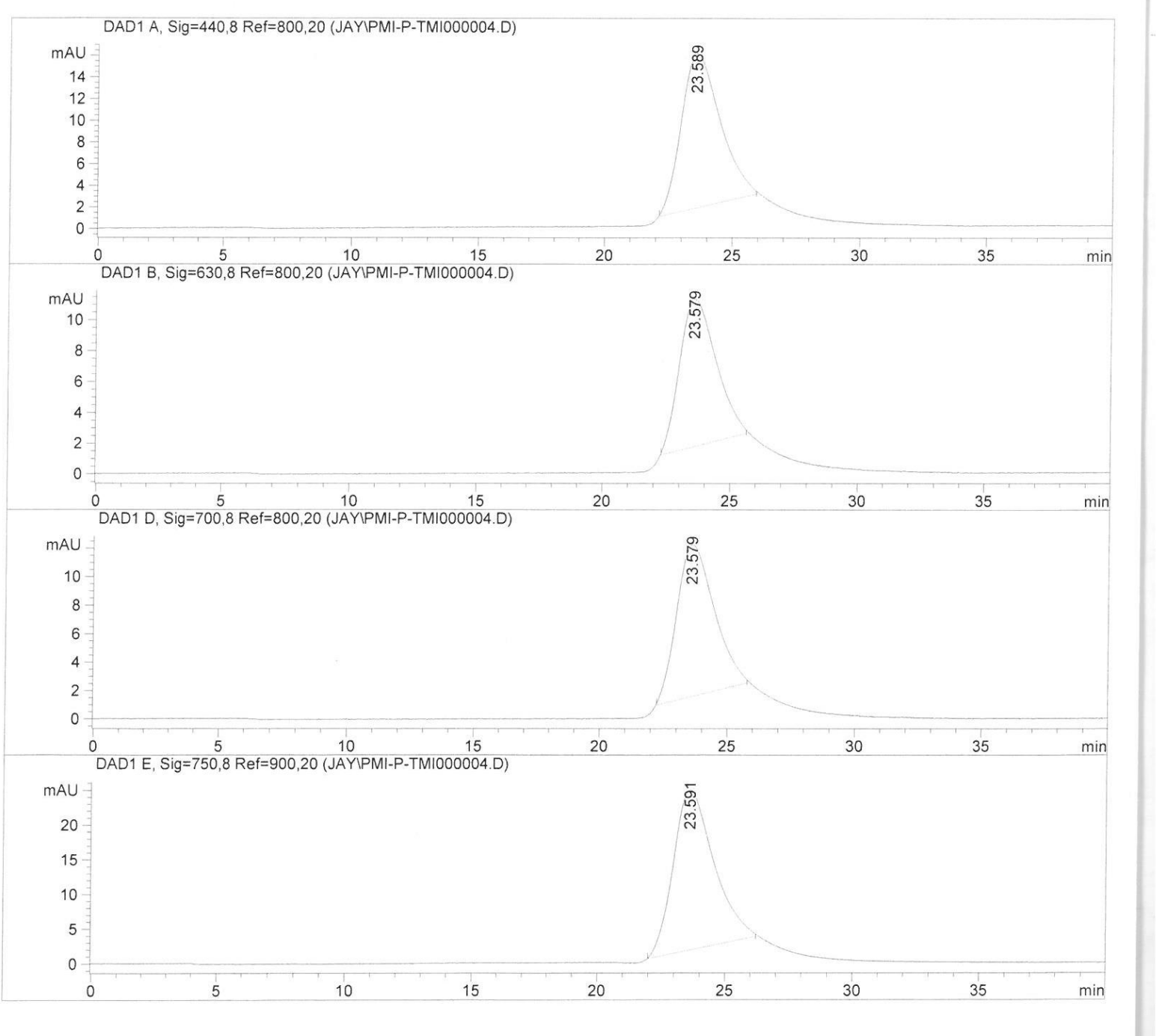

Figure S25. Analytical SEC trace of compound PMI-P-TMI. 


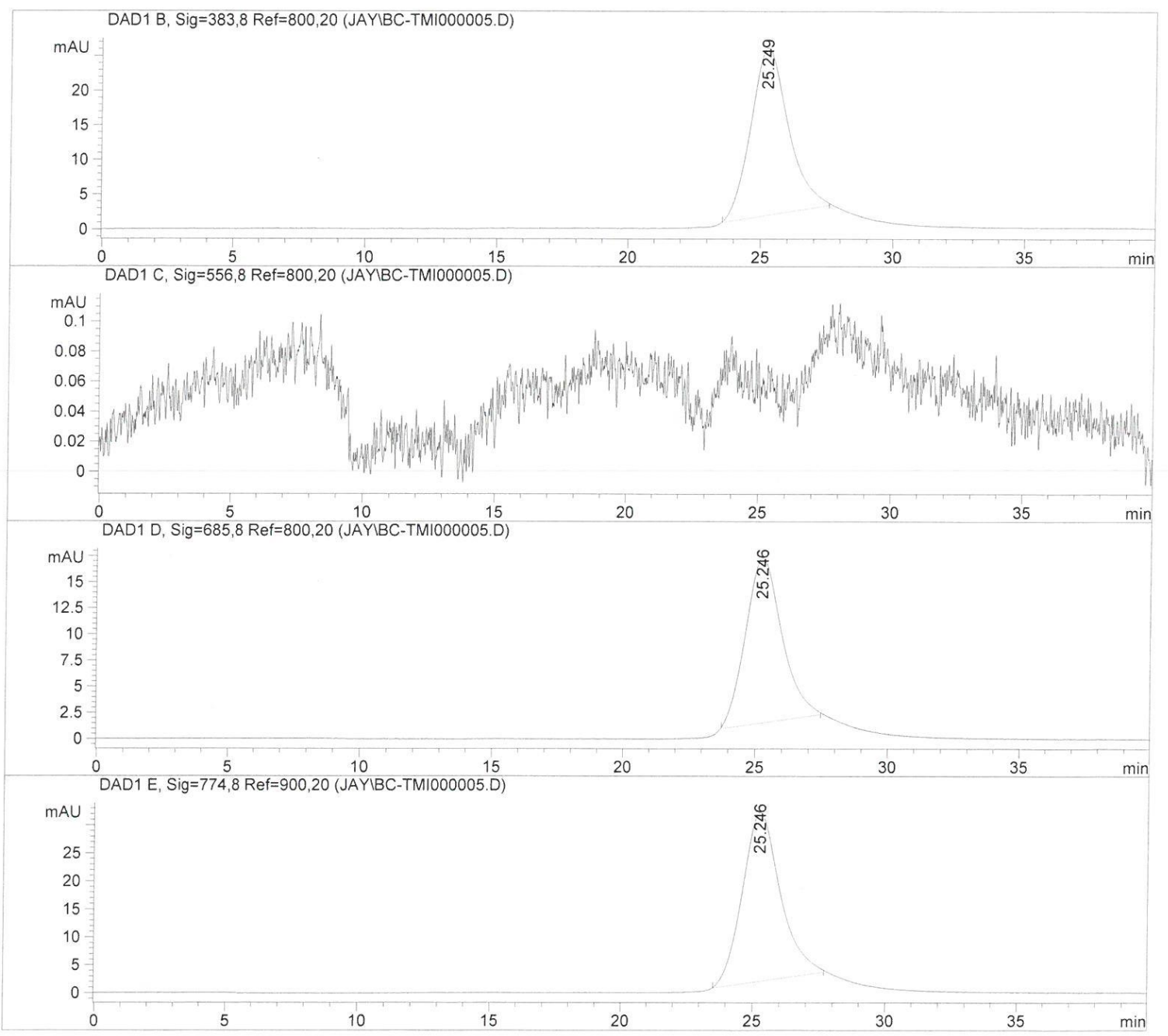

Figure S26. Analytical SEC trace of compound B-TMI. 


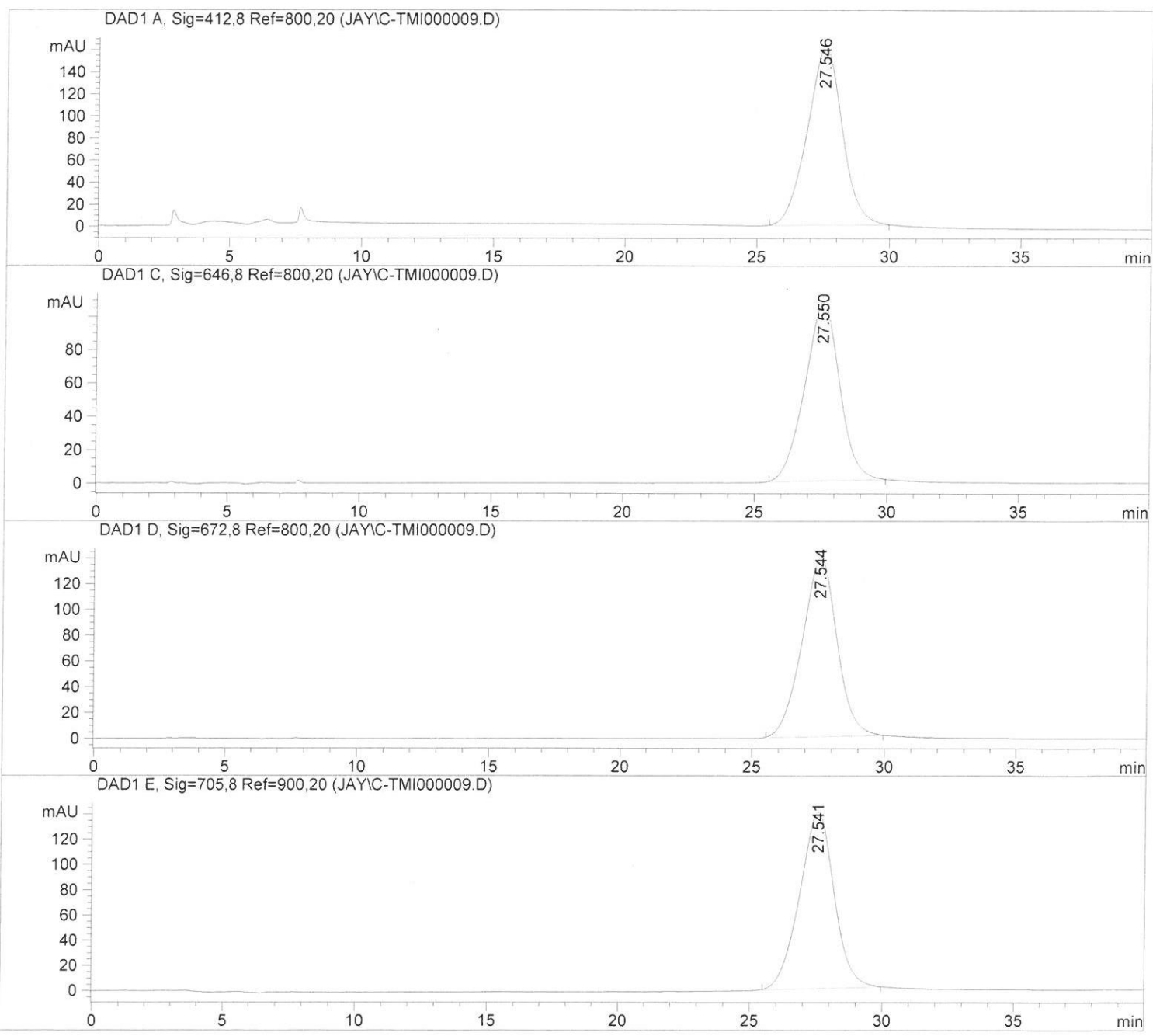

Figure S27. Analytical SEC trace of compound C-TMI. 


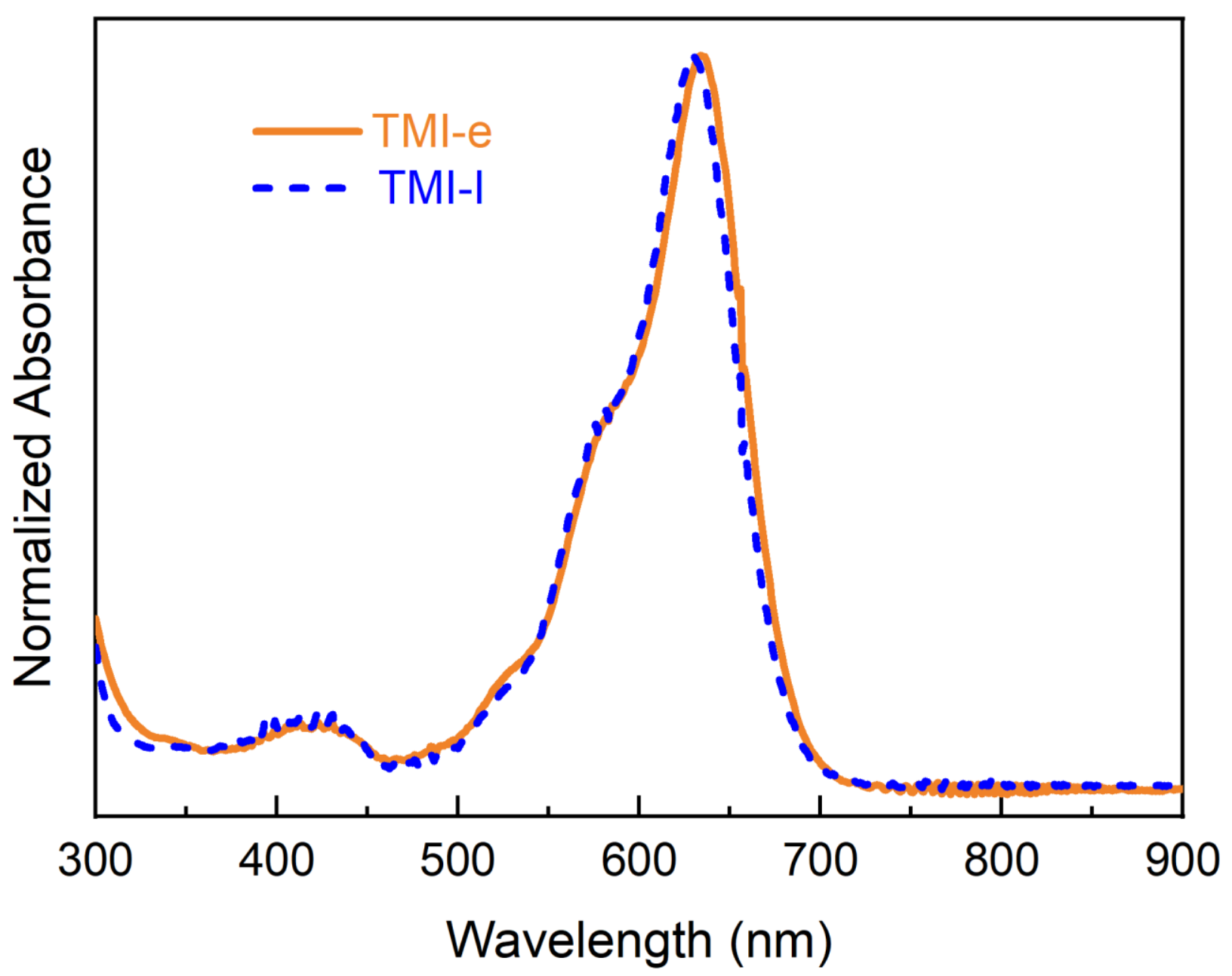

Figure S28. Normalized absorption spectra of TMI-I and TMI-e in toluene. 
Section S2

\author{
Spectral \\ Properties
}


Table S1. Spectral Properties of Arrays and Benchmark Monomers in Benzonitrile. ${ }^{a}$

\begin{tabular}{|c|c|c|c|c|c|c|c|c|c|c|c|c|c|c|}
\hline Compound & $\begin{array}{l}\lambda_{\mathrm{abs} 1} \\
(\mathrm{~nm})\end{array}$ & $\mathrm{I}_{1}$ & $\begin{array}{l}\lambda_{\text {abs2 }} \\
(\mathrm{nm})\end{array}$ & $\mathrm{I}_{2}$ & $\begin{array}{l}\lambda_{\text {abs3 }} \\
(\mathrm{nm})\end{array}$ & $\mathrm{I}_{3}$ & $\begin{array}{l}\lambda_{\mathrm{abs} 4} \\
(\mathrm{~nm})\end{array}$ & $\mathrm{I}_{4}$ & $\begin{array}{l}\lambda_{\text {abs5 }} \\
(\mathrm{nm})\end{array}$ & $\mathrm{I}_{5}$ & $\begin{array}{l}\lambda_{\text {abs6 }} \\
(\mathrm{nm})\end{array}$ & $\mathrm{I}_{6}$ & $\begin{array}{c}\lambda_{\mathrm{em}} \\
(\mathrm{nm})\end{array}$ & $\begin{array}{c}\Delta_{\mathrm{ab}-\mathrm{em}} \\
\left(\mathrm{cm}^{-1}\right)^{b}\end{array}$ \\
\hline \multicolumn{15}{|l|}{ New Arrays } \\
\hline PMI-P-TMI & $408^{c}$ & 0.41 & 442 & 0.60 & $479^{c}$ & 0.34 & 544 & 0.42 & 663 & 0.62 & 763 & 1.00 & 799 & 591 \\
\hline C-TMI & 415 & 1.00 & $435^{c}$ & 0.67 & 462 & 0.34 & 611 & 0.32 & 674 & 0.79 & 711 & 0.87 & 833 & 2060 \\
\hline B-TMI & 387 & 0.95 & $442^{c}$ & 0.14 & 560 & 0.18 & 617 & 0.36 & 690 & 0.76 & 778 & 1.00 & 778 & 10 \\
\hline \multicolumn{15}{|l|}{ Prior Arrays } \\
\hline P-TMI $^{d}$ & & & 426 & 1.0 & & & 535 & 0.11 & 650 & 0.42 & 726 & 0.57 & 768 & 753 \\
\hline P-PMI ${ }^{e}$ & & & 434 & 1.0 & 497 & 0.27 & 534 & 0.48 & 616 & 0.51 & 691 & 0.47 & 695 & 83 \\
\hline C-PMI ${ }^{f}$ & 394 & 0.60 & 433 & 1.0 & 524 & 0.41 & 603 & 0.55 & 642 & 0.56 & 673 & 0.65 & 750 & 1525 \\
\hline B-PMI ${ }^{f}$ & & & 384 & 0.98 & 515 & 0.28 & & & 622 & 0.61 & 772 & 1.00 & 784 & 198 \\
\hline PMI-P-PMI $^{e}$ & & & 438 & 0.70 & 480 & 0.59 & 540 & 0.89 & 642 & 0.70 & 738 & 1.00 & 757 & 340 \\
\hline \multicolumn{15}{|l|}{ Benchmarks } \\
\hline $\mathbf{P}-\mathbf{P h}{ }^{f}$ & & & 437 & 1.00 & 535 & 0.033 & 577 & 0.082 & 611 & 0.017 & 669 & 0.032 & 674 & 110 \\
\hline $\mathbf{C}-\mathbf{P h}{ }^{f}$ & 383 & 0.20 & 430 & 1.00 & 525 & 0.053 & 569 & 0.12 & 607 & 0.054 & 661 & 0.18 & 663 & 45 \\
\hline $\mathbf{B}-\mathbf{P h}{ }^{f}$ & & & 391 & 0.99 & 518 & 0.089 & 554 & 0.34 & 690 & 0.061 & 757 & 1.00 & 766 & 155 \\
\hline PMI-Ph ${ }^{d}$ & & & & & $511^{c}$ & 0.69 & 541 & 1.00 & & & & & 590 & 1535 \\
\hline $\mathbf{T M I}-\mathbf{P h}^{d}$ & & & 432 & 0.10 & & & 658 & 1.00 & & & & & 743 & 1739 \\
\hline
\end{tabular}

${ }^{a}$ All measurements were made at room temperature. Listed for each band are the wavelength $\left(\lambda_{\mathrm{i}}\right)$ and the intensity ( $\left.\mathrm{I}_{\mathrm{i}}\right)$ relative to the largest peak in the spectrum for that compound. The bands for various compounds are listed by columns corresponding to the same general wavelength position and are not meant to necessarily imply a common electronic transition. ${ }^{b}$ The (Stokes) shift between the longest wavelength absorption peak and the emission maximum. ${ }^{c}$ Pronounced shoulder. ${ }^{d}$ From ref. 16. ${ }^{e}$ From ref. $18 .{ }^{f}$ From ref. 13. 


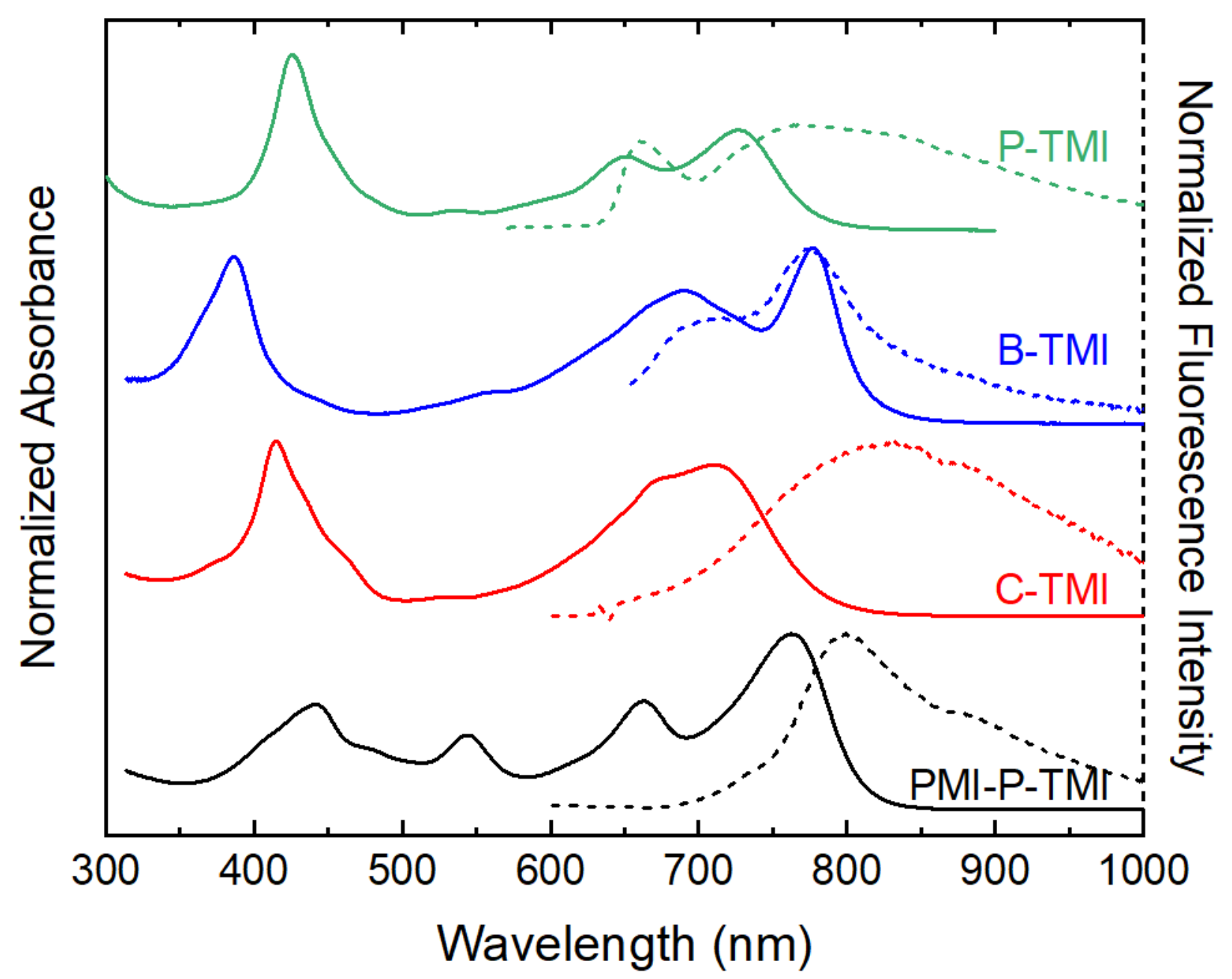

Figure S29. Absorption and fluorescence spectra of new arrays in benzonitrile. 


\title{
Section S3
}

\author{
Transient \\ Absorption \\ Data
}


Table S2. Summary of Kinetic Analysis of TA Data for Arrays from Global Analysis. ${ }^{a}$

\begin{tabular}{|c|c|c|c|c|c|c|c|c|c|}
\hline \multirow[t]{2}{*}{ Compound } & \multirow[b]{2}{*}{$\begin{array}{c}\lambda_{\mathrm{ex}} \\
(\mathrm{nm}) \\
\end{array}$} & \multicolumn{2}{|c|}{ Tol (DADS) } & \multicolumn{2}{|c|}{ Tol (EADS) } & \multicolumn{2}{|c|}{ PhCN (DADS) } & \multicolumn{2}{|c|}{$\mathrm{PhCN}$ (DADS) } \\
\hline & & $\begin{array}{c}\tau_{1} \\
\text { (ps) }\end{array}$ & $\begin{array}{c}\tau_{2} \\
\text { (ps) }\end{array}$ & $\begin{array}{c}\tau_{1} \\
(\mathrm{ps})\end{array}$ & $\begin{array}{c}\tau_{2} \\
(\mathrm{ps})\end{array}$ & $\begin{array}{c}\tau_{1} \\
\text { (ps) }\end{array}$ & $\begin{array}{c}\tau_{2} \\
\text { (ps) }\end{array}$ & $\begin{array}{c}\tau_{1} \\
(\mathrm{ps})\end{array}$ & $\begin{array}{c}\tau_{2} \\
\text { (ps) }\end{array}$ \\
\hline PMI-P-TMI & 440 & 29 & 139 & 44 & 136 & 24 & 98 & 24 & 95 \\
\hline \multirow{3}{*}{ (batch 1) } & 540 & 29 & 139 & 35 & 142 & 25 & 97 & 26 & 97 \\
\hline & 660 & 30 & 139 & 35 & 142 & 24 & 100 & 23 & 98 \\
\hline & 750 & 28 & 139 & 32 & 139 & 25 & 93 & 25 & 93 \\
\hline Average & & 29 & 139 & 37 & 140 & 25 & 97 & 25 & 96 \\
\hline Std Dev & & 1 & 0 & 5 & 3 & 1 & 3 & 1 & 2 \\
\hline PMI-P-TMI & 440 & 37 & 138 & 39 & 140 & 22 & 97 & 25 & 100 \\
\hline \multirow[t]{3}{*}{ (batch 2) } & 540 & 34 & 140 & 43 & 139 & 33 & 99 & 30 & 98 \\
\hline & 660 & 28 & 148 & 24 & 142 & 24 & 101 & 24 & 102 \\
\hline & 750 & 39 & 146 & 38 & 142 & 24 & 94 & 26 & 93 \\
\hline Average & & 35 & 143 & 36 & 141 & 26 & 98 & 26 & 98 \\
\hline Std Dev & & 5 & 5 & 8 & 2 & 5 & 3 & 3 & 4 \\
\hline \multirow[t]{3}{*}{ C-TMI } & 411 & 17 & 132 & 16 & 130 & 18 & 67 & 20 & 65 \\
\hline & 670 & 20 & 133 & 22 & 132 & 14 & 66 & 13 & 70 \\
\hline & 705 & 18 & 120 & 20 & 119 & 12 & 65 & 12 & 67 \\
\hline Average & & 18 & 128 & 19 & 127 & 15 & 66 & 15 & 67 \\
\hline Std Dev & & 2 & 7 & 3 & 7 & 3 & 1 & 4 & 3 \\
\hline \multirow[t]{3}{*}{ B-TMI } & 385 & 30 & 384 & 29 & 379 & 4 & 15 & 2 & 17 \\
\hline & 680 & 31 & 371 & 31 & 371 & 5 & 14 & 6 & 12 \\
\hline & 774 & 36 & 375 & 37 & 376 & 4 & 14 & 6 & 12 \\
\hline Average & & 32 & 377 & 32 & 375 & 4 & 14 & 5 & 14 \\
\hline Std Dev & & 3 & 7 & 4 & 4 & 1 & 1 & 2 & 3 \\
\hline \multirow[t]{3}{*}{ P-TMI } & 425 & 15 & 298 & 17 & 302 & & & & \\
\hline & 645 & 13 & 300 & 13 & 301 & 29 & 141 & 28 & 139 \\
\hline & 715 & 16 & 278 & 14 & 277 & & & & \\
\hline Average & & 15 & 292 & 15 & 293 & 29 & 141 & 28 & 139 \\
\hline Std Dev & & 2 & 12 & 2 & 14 & & & & \\
\hline
\end{tabular}

${ }^{a}$ Samples were excited with $\sim 100$ fs excitation flashes at the wavelengths indicated. Global analysis gave Decay Associated Difference Spectra (DADS) by employing two independent exponential components or Evolution Associated Difference Spectra (EADS) by employing a sequential model with two decay components. Raw TA spectra, DADS and EADS are given in Figures S30-S41. Average time constants from the global analysis (EADS and DADS) for each array are given in Table 1 of the paper. 

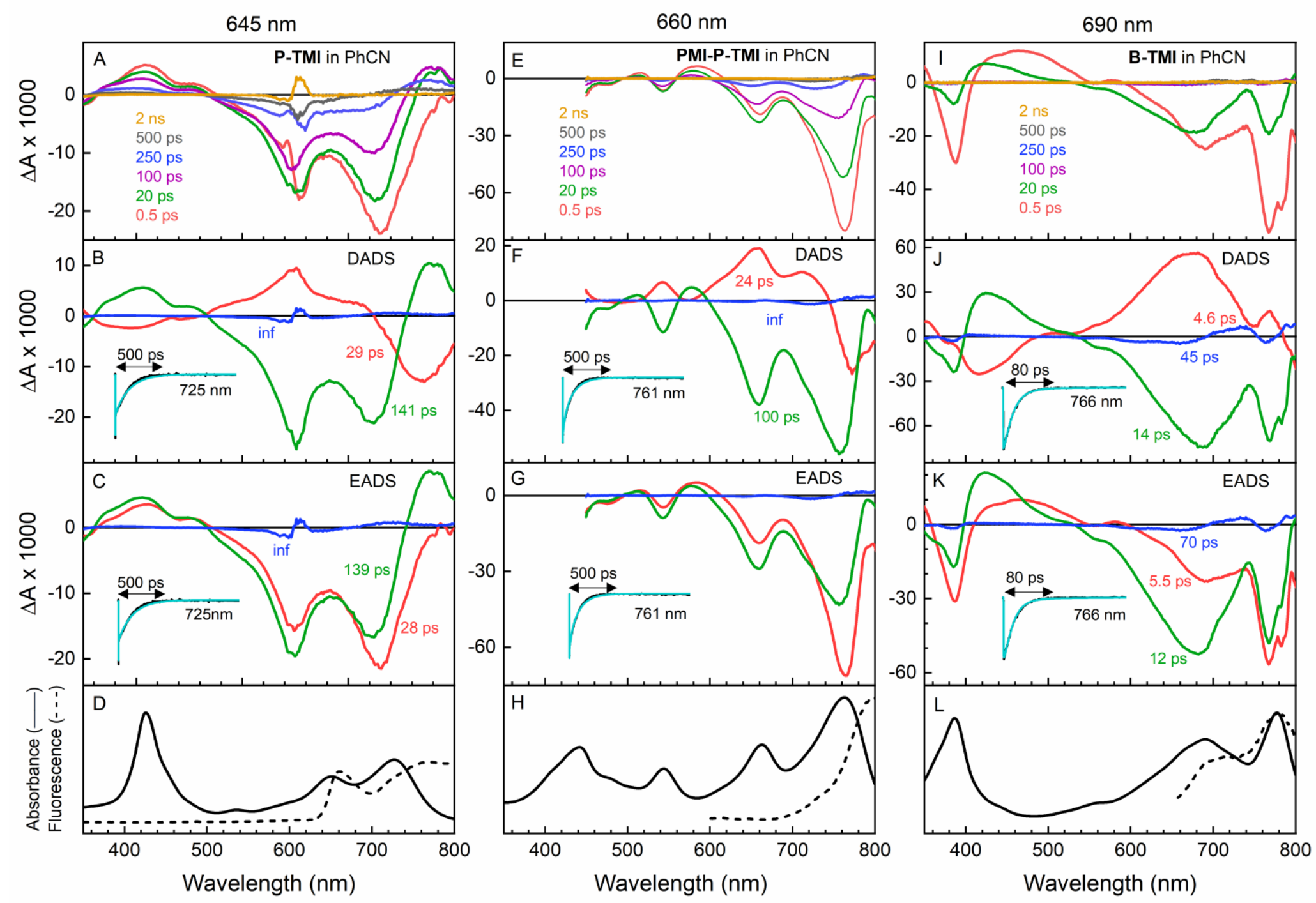

Figure S30. TA results for P-TMI (left), PMI-P-TMI (middle) and C-TMI (right) in benzonitrile. (Top row) Representative TA spectra at indicated delay times after excitation at the wavelength indicated at the top; (Second row) Decay associated difference spectra (DADS) obtained from global fitting of TA data; (Third row) Evolution associated difference spectra (EADS) obtained from global fitting of TA data. Insets are kinetic traces (black) at the indicated wavelength and corresponding fits (cyan); (Bottom row) Ground state absorption (solid) and fluorescence emission (dashed) spectra. 

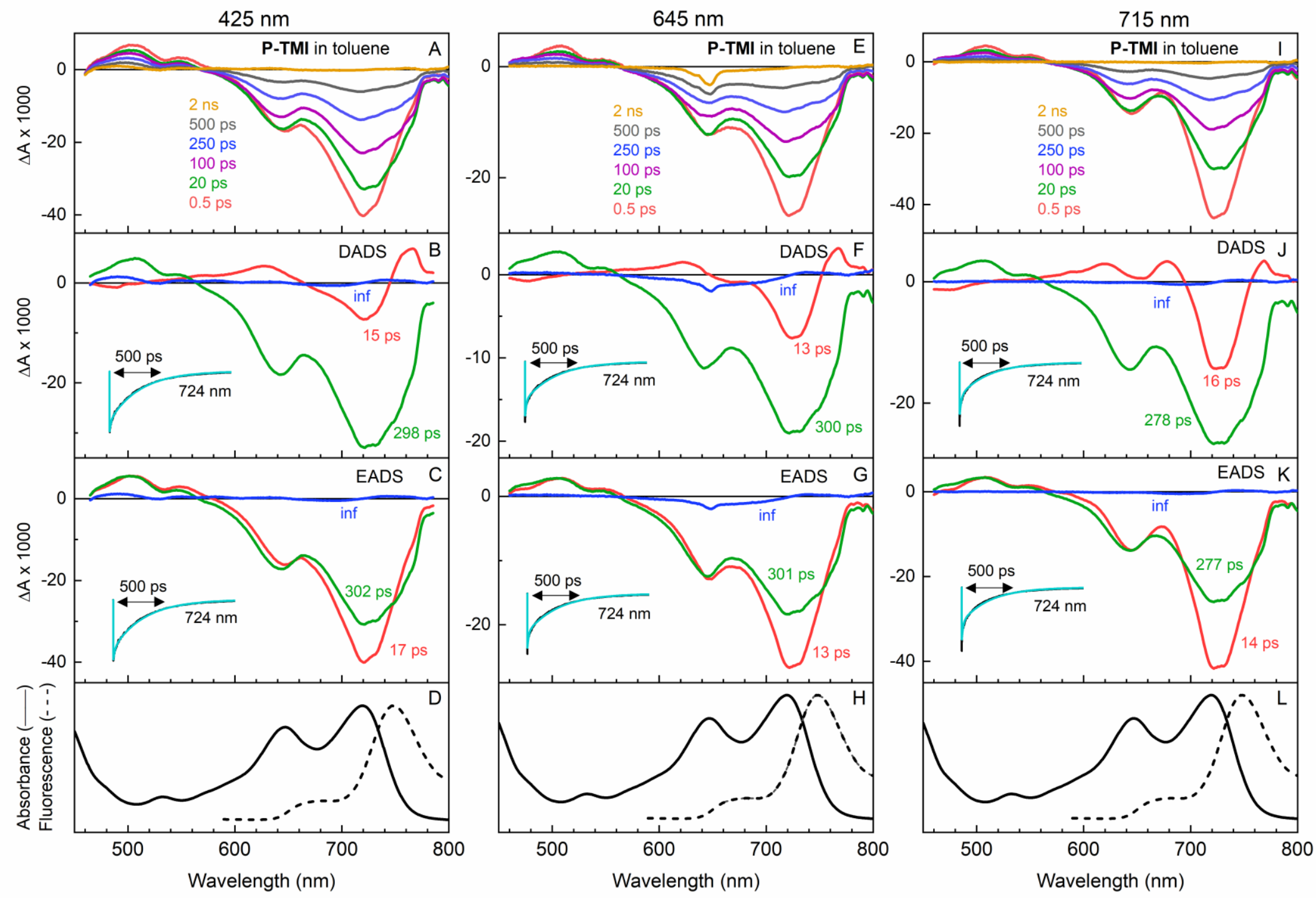

Figure S31. TA data for P-TMI in toluene using excitation at three different wavelengths. (Top row) Representative TA spectra at indicated delay times after excitation at the wavelength indicated at the top; (Second row) Decay associated difference spectra (DADS) obtained from global fitting of TA data; (Third row) Evolution associated difference spectra (EADS) obtained from global fitting of TA data. Insets are kinetic traces (black) at the indicated wavelength and corresponding fits (cyan); (Bottom row) Ground state absorption (solid) and fluorescence emission (dashed) spectra. 


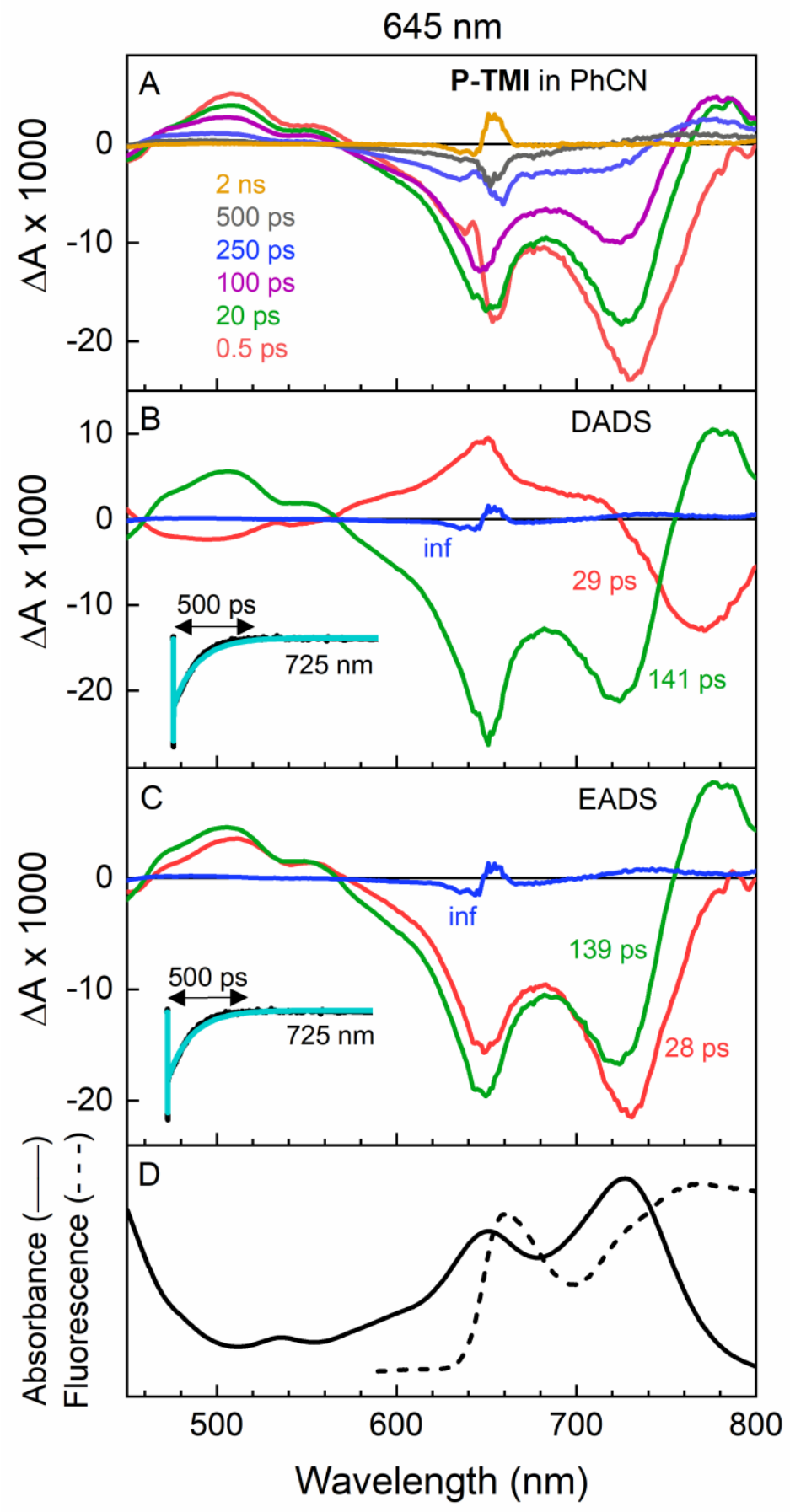

Figure S32. TA data for P-TMI in benzonitrile ( $\mathrm{PhCN}$ ) using excitation at $645 \mathrm{~nm}$. (Top row) Representative TA spectra at indicated delay times after excitation at the wavelength indicated at the top; (Second row) Decay associated difference spectra (DADS) obtained from global fitting of TA data; (Third row) Evolution associated difference spectra (EADS) obtained from global fitting of TA data. Insets are kinetic traces (black) at the indicated wavelength and corresponding fits (cyan); (Bottom row) Ground state absorption (solid) and fluorescence emission (dashed) spectra. 

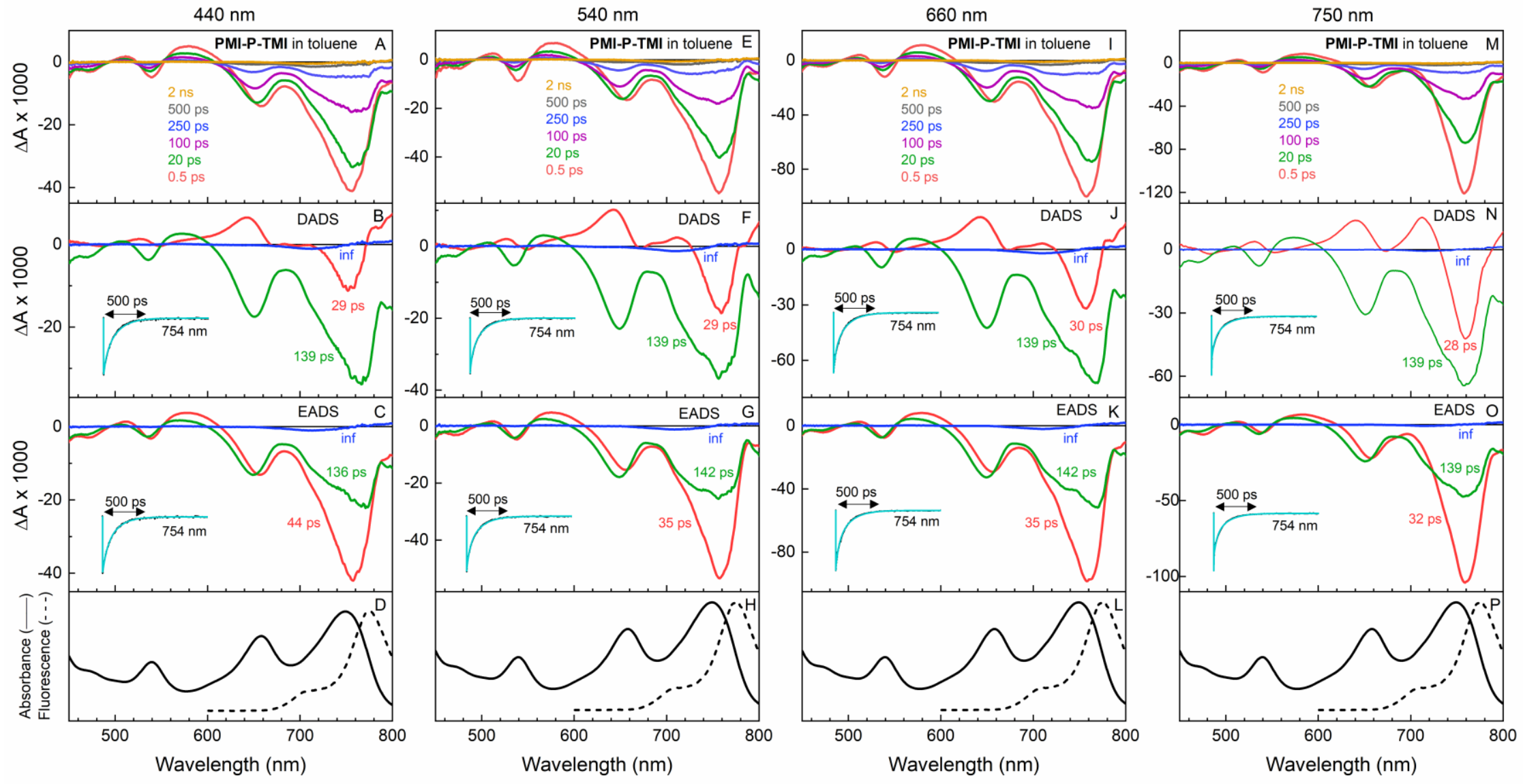

Figure S33. TA data for PMI-P-TMI (batch 1) in toluene using excitation at four different wavelengths. (Top row) Representative TA spectra at indicated delay times after excitation at the wavelength indicated at the top; (Second row) Decay associated difference spectra (DADS) obtained from global fitting of TA data; (Third row) Evolution associated difference spectra (EADS) obtained from global fitting of TA data. Insets are kinetic traces (black) at the indicated wavelength and corresponding fits (cyan); (Bottom row) Ground state absorption (solid) and fluorescence emission (dashed) spectra. 

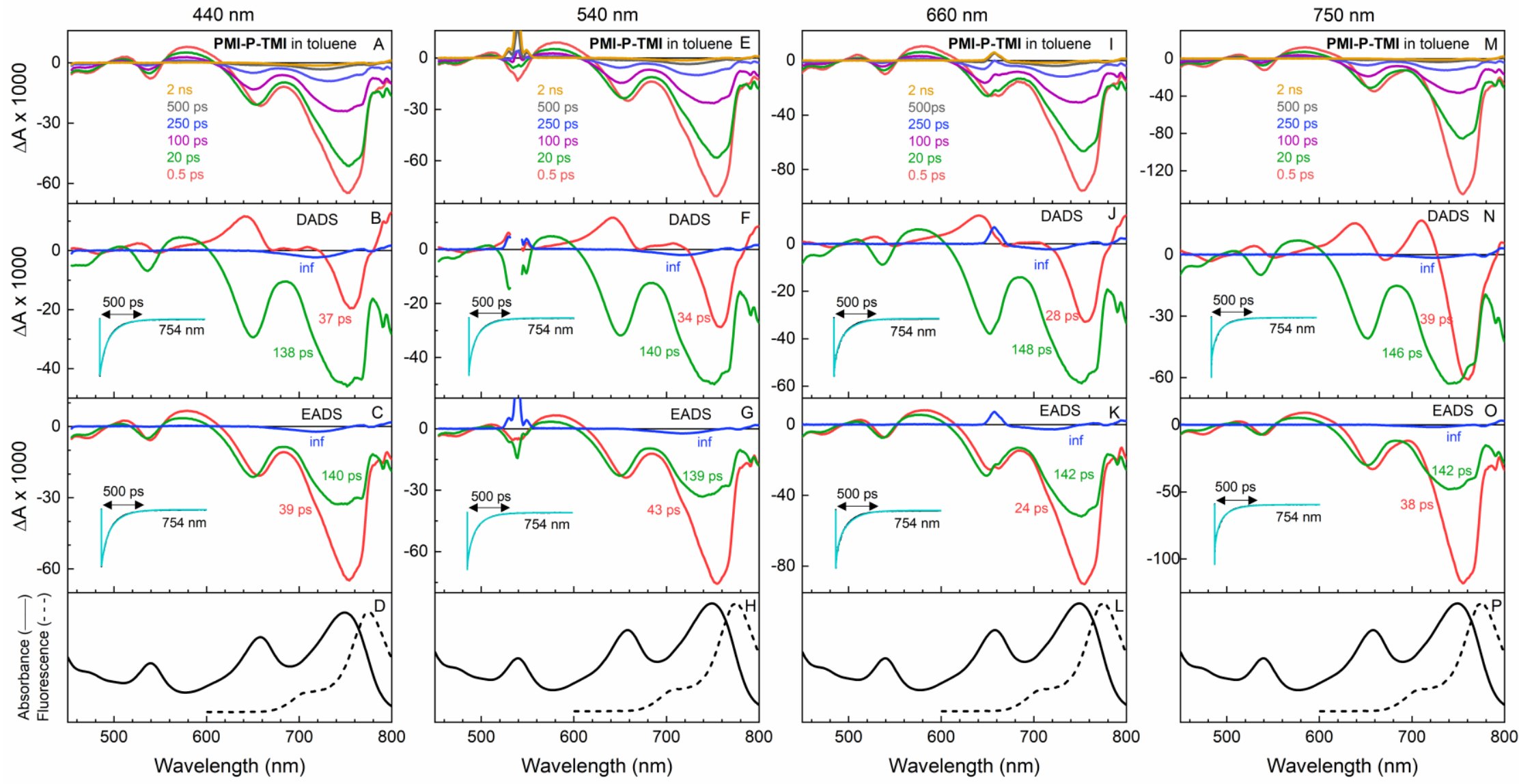

Figure S34. TA data for PMI-P-TMI (batch 2) in toluene using excitation at four different wavelengths. (Top row) Representative TA spectra at indicated delay times after excitation at the wavelength indicated at the top; (Second row) Decay associated difference spectra (DADS) obtained from global fitting of TA data; (Third row) Evolution associated difference spectra (EADS) obtained from global fitting of TA data. Insets are kinetic traces (black) at the indicated wavelength and corresponding fits (cyan); (Bottom row) Ground state absorption (solid) and fluorescence emission (dashed) spectra. 

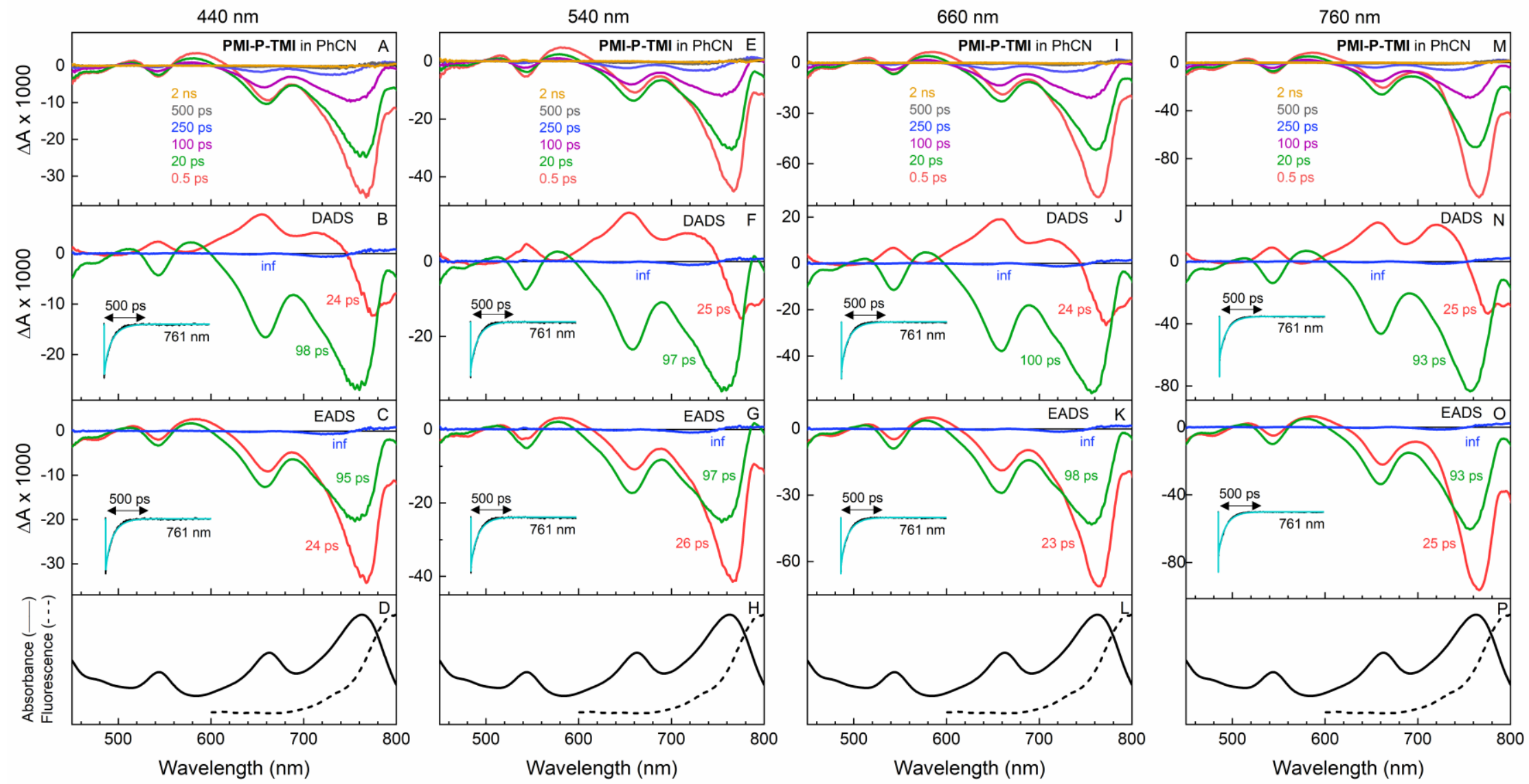

Figure S35. TA data for PMI-P-TMI (batch 1) in benzonitrile (PhCN) using excitation at four different wavelengths. (Top row) Representative TA spectra at indicated delay times after excitation at the wavelength indicated at the top; (Second row) Decay associated difference spectra (DADS) obtained from global fitting of TA data; (Third row) Evolution associated difference spectra (EADS) obtained from global fitting of TA data. Insets are kinetic traces (black) at the indicated wavelength and corresponding fits (cyan); (Bottom row) Ground state absorption (solid) and fluorescence emission (dashed) spectra. 

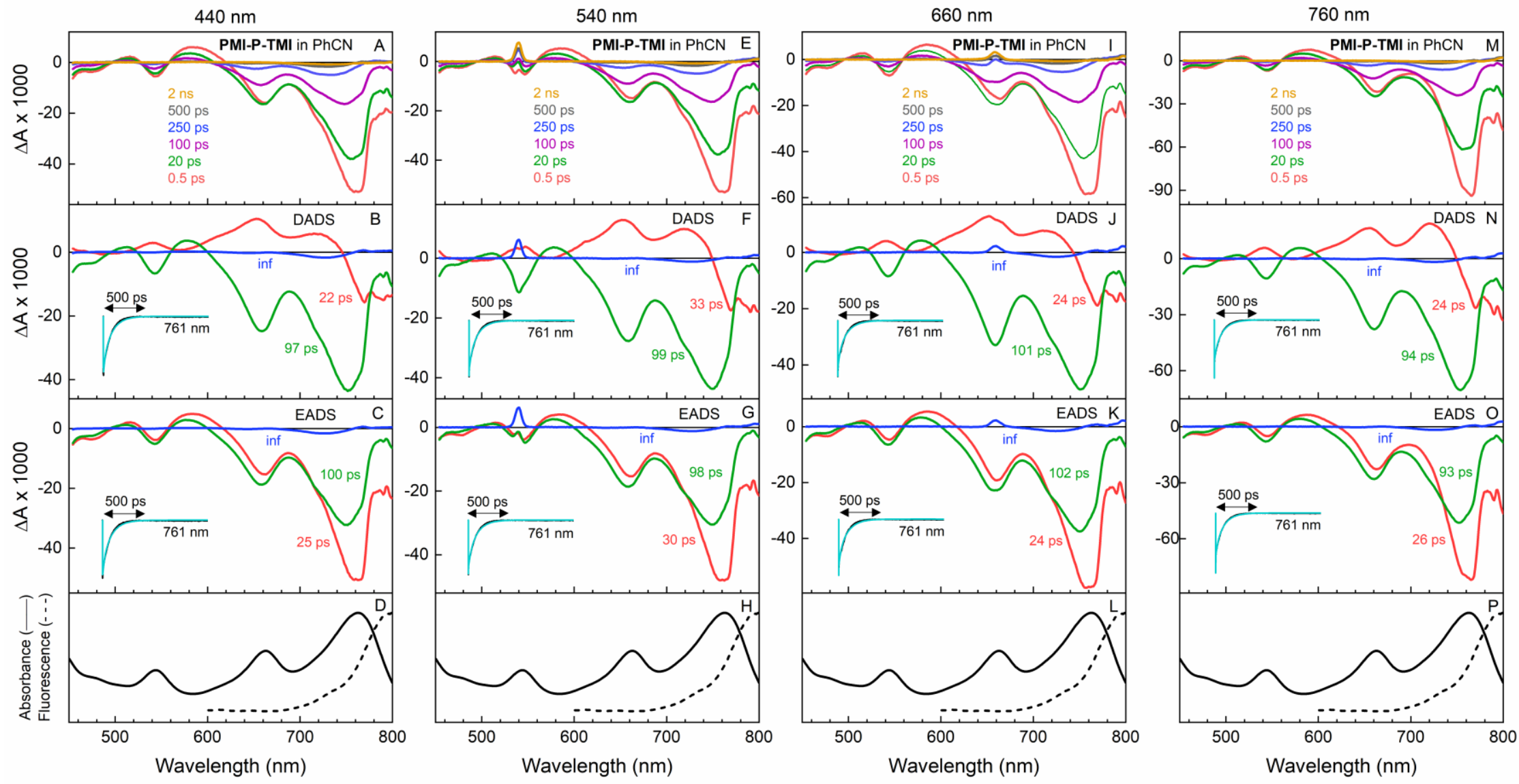

Figure S36. TA data for PMI-P-TMI (batch 2) in benzonitrile (PhCN) using excitation at four different wavelengths. (Top row) Representative TA spectra at indicated delay times after excitation at the wavelength indicated at the top; (Second row) Decay associated difference spectra (DADS) obtained from global fitting of TA data; (Third row) Evolution associated difference spectra (EADS) obtained from global fitting of TA data. Insets are kinetic traces (black) at the indicated wavelength and corresponding fits (cyan); (Bottom row) Ground state absorption (solid) and fluorescence emission (dashed) spectra. 

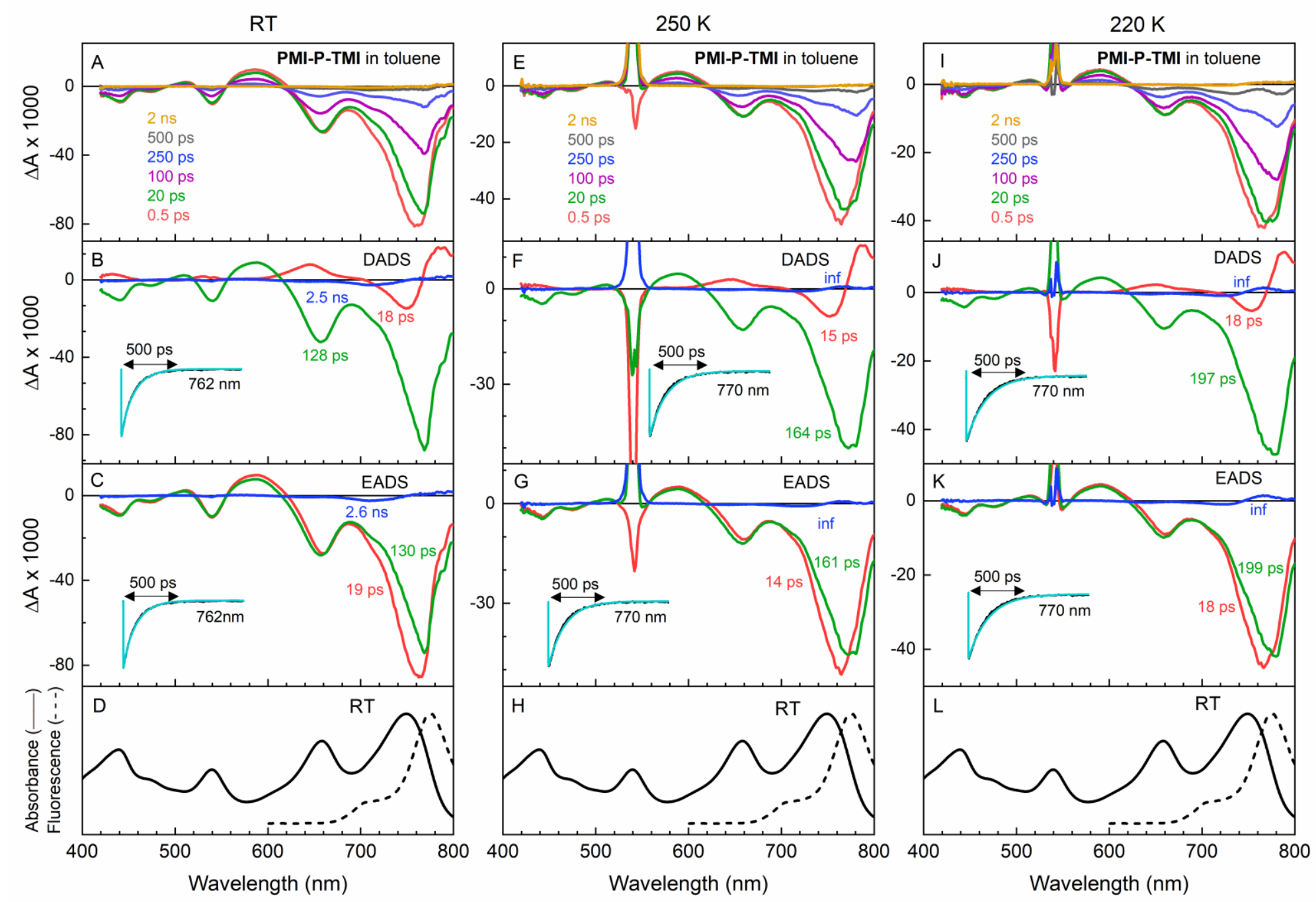

Figure S37. TA data for PMI-P-TMI (batch 1) in toluene using excitation at $540 \mathrm{~nm}$ at three temperatures. (Top row) Representative TA spectra at indicated delay times after excitation at the wavelength indicated at the top; (Second row) Decay associated difference spectra (DADS) obtained from global fitting of TA data; (Third row) Evolution associated difference spectra (EADS) obtained from global fitting of TA data. Insets are kinetic traces (black) at the indicated wavelength and corresponding fits (cyan); (Bottom row) Ground state absorption (solid) and fluorescence emission (dashed) spectra at room temperature (295 K). 

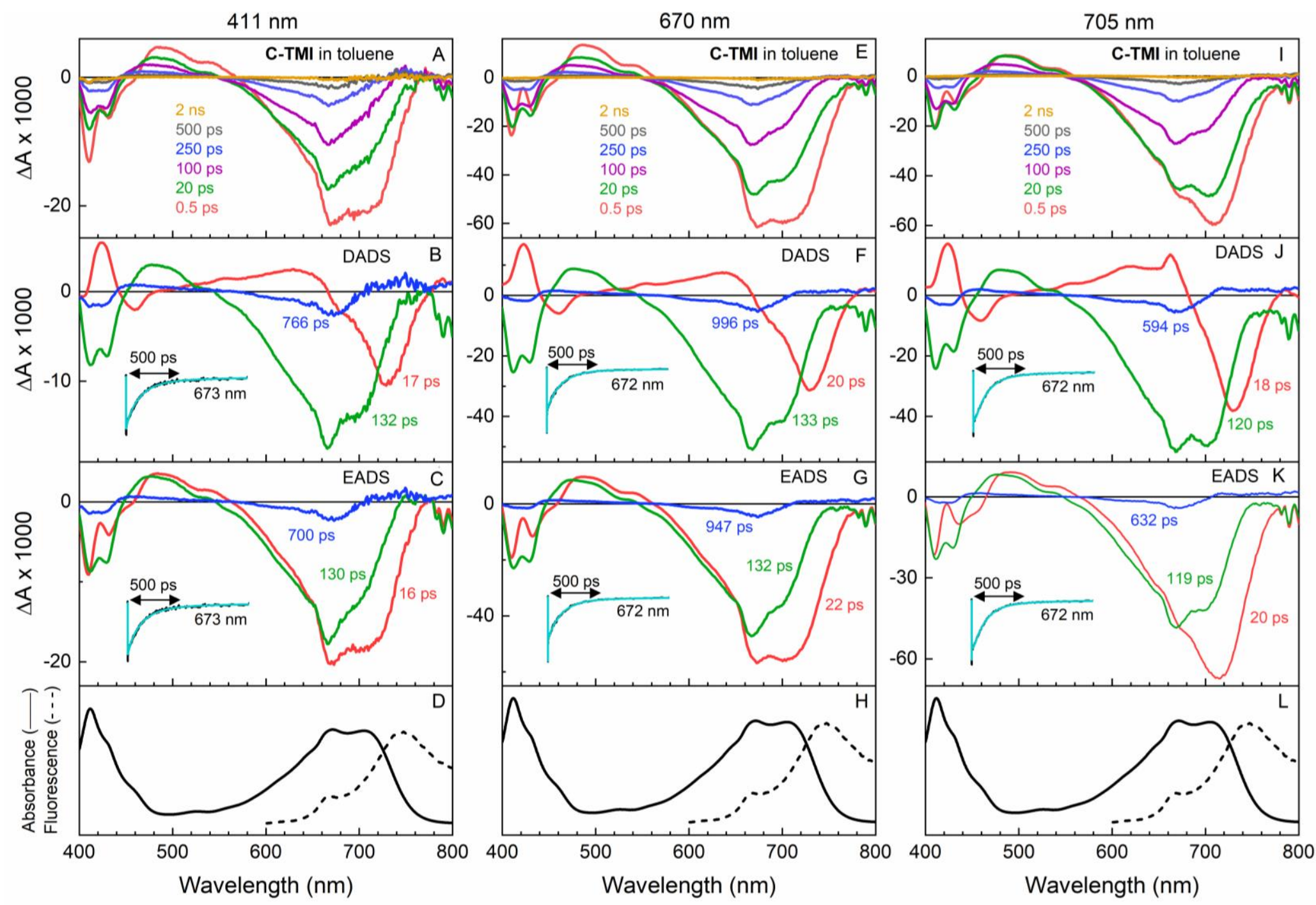

Figure S38. TA data for C-TMI in toluene using excitation at three different wavelengths. (Top row) Representative TA spectra at indicated delay times after excitation at the wavelength indicated at the top; (Second row) Decay associated difference spectra (DADS) obtained from global fitting of TA data; (Third row) Evolution associated difference spectra (EADS) obtained from global fitting of TA data. Insets are kinetic traces (black) at the indicated wavelength and corresponding fits (cyan); (Bottom row) Ground state absorption (solid) and fluorescence emission (dashed) spectra. 

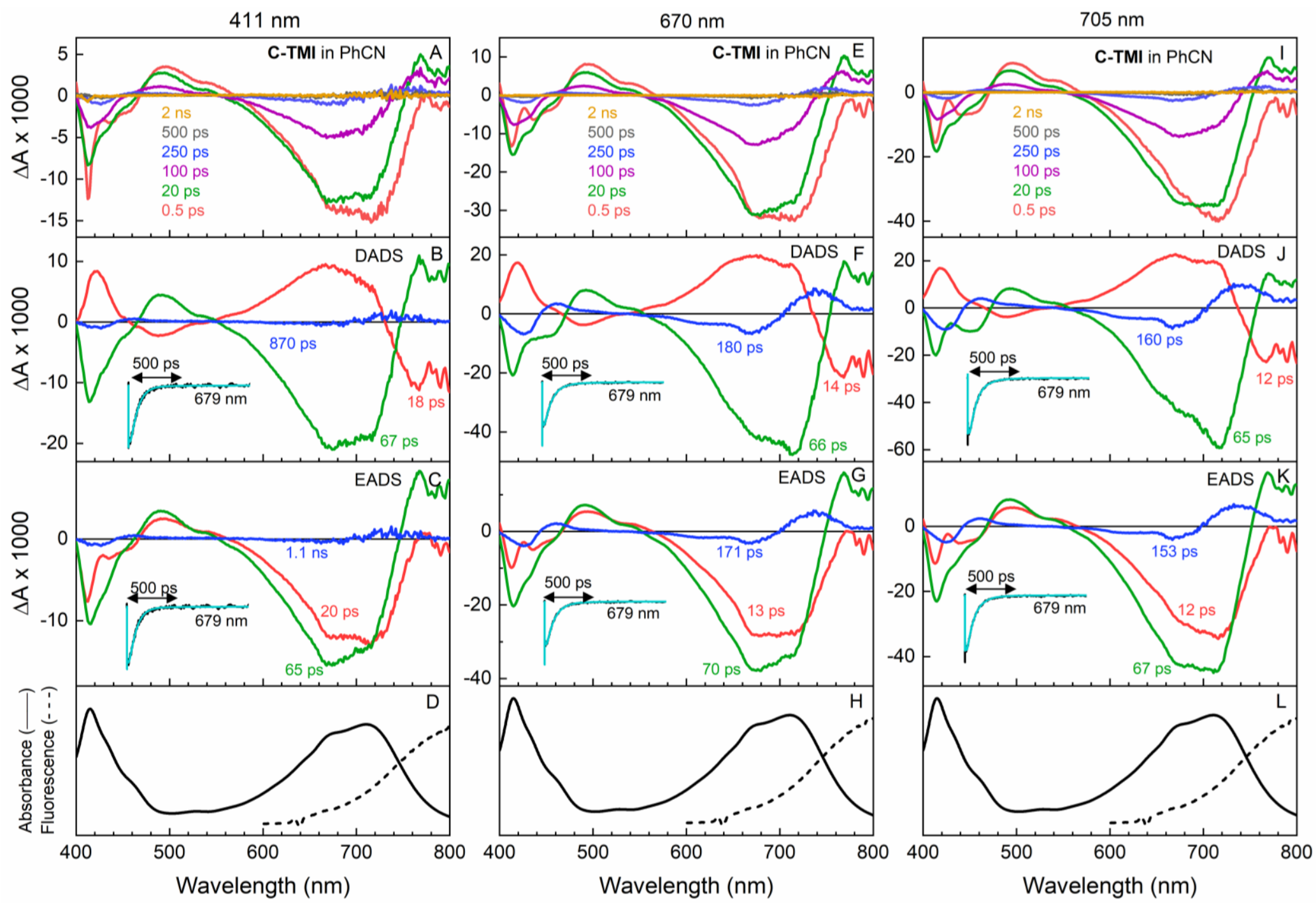

Figure S39. TA data for C-TMI in benzonitrile $(\mathrm{PhCN})$ using excitation at three different wavelengths. (Top row) Representative TA spectra at indicated delay times after excitation at the wavelength indicated at the top; (Second row) Decay associated difference spectra (DADS) obtained from global fitting of TA data; (Third row) Evolution associated difference spectra (EADS) obtained from global fitting of TA data. Insets are kinetic traces (black) at the indicated wavelength and corresponding fits (cyan); (Bottom row) Ground state absorption (solid) and fluorescence emission (dashed) spectra. 

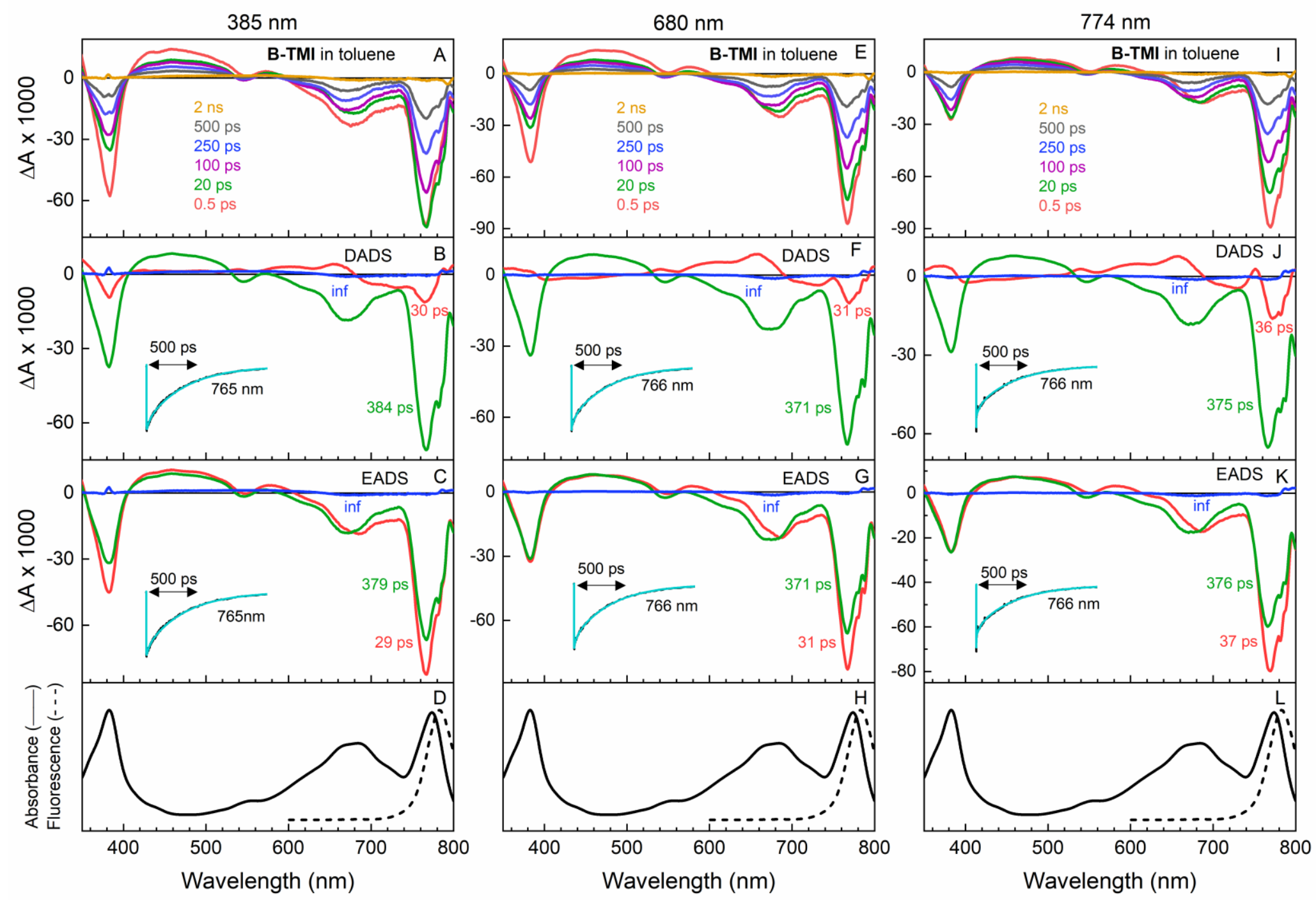

Figure S40. TA data for B-TMI in toluene using excitation at three different wavelengths. (Top row) Representative TA spectra at indicated delay times after excitation at the wavelength indicated at the top; (Second row) Decay associated difference spectra (DADS) obtained from global fitting of TA data; (Third row) Evolution associated difference spectra (EADS) obtained from global fitting of TA data. Insets are kinetic traces (black) at the indicated wavelength and corresponding fits (cyan); (Bottom row) Ground state absorption (solid) and fluorescence emission (dashed) spectra. 

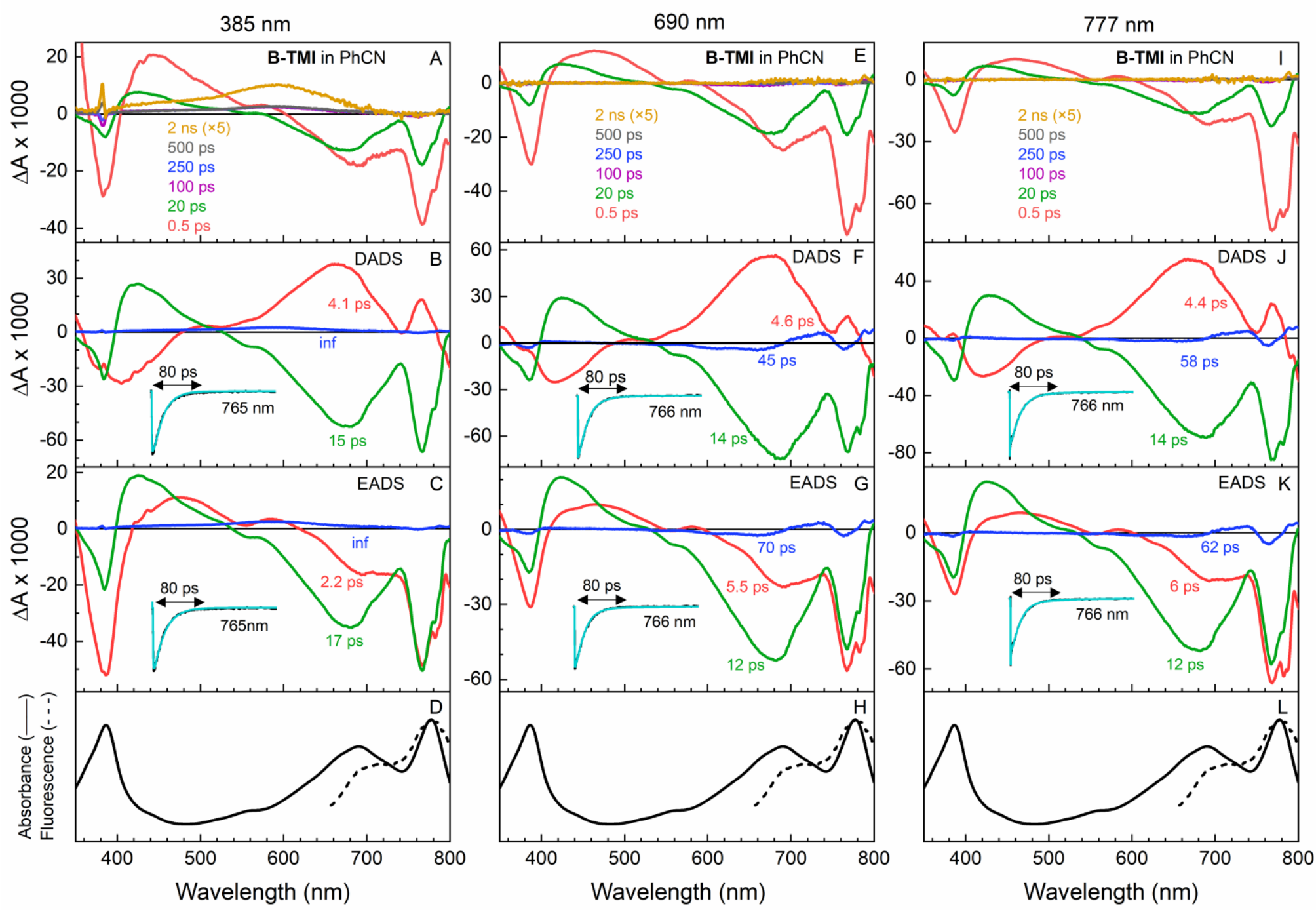

Figure S41. TA data for B-TMI in benzonitrile ( $\mathrm{PhCN}$ ) using excitation at three different wavelengths. (Top row) Representative TA spectra at indicated delay times after excitation at the wavelength indicated at the top; (Second row) Decay associated difference spectra (DADS) obtained from global fitting of TA data; (Third row) Evolution associated difference spectra (EADS) obtained from global fitting of TA data. Insets are kinetic traces (black) at the indicated wavelength and corresponding fits (cyan); (Bottom row) Ground state absorption (solid) and fluorescence emission (dashed) spectra. 
Section S4

\section{Molecular Orbitals}




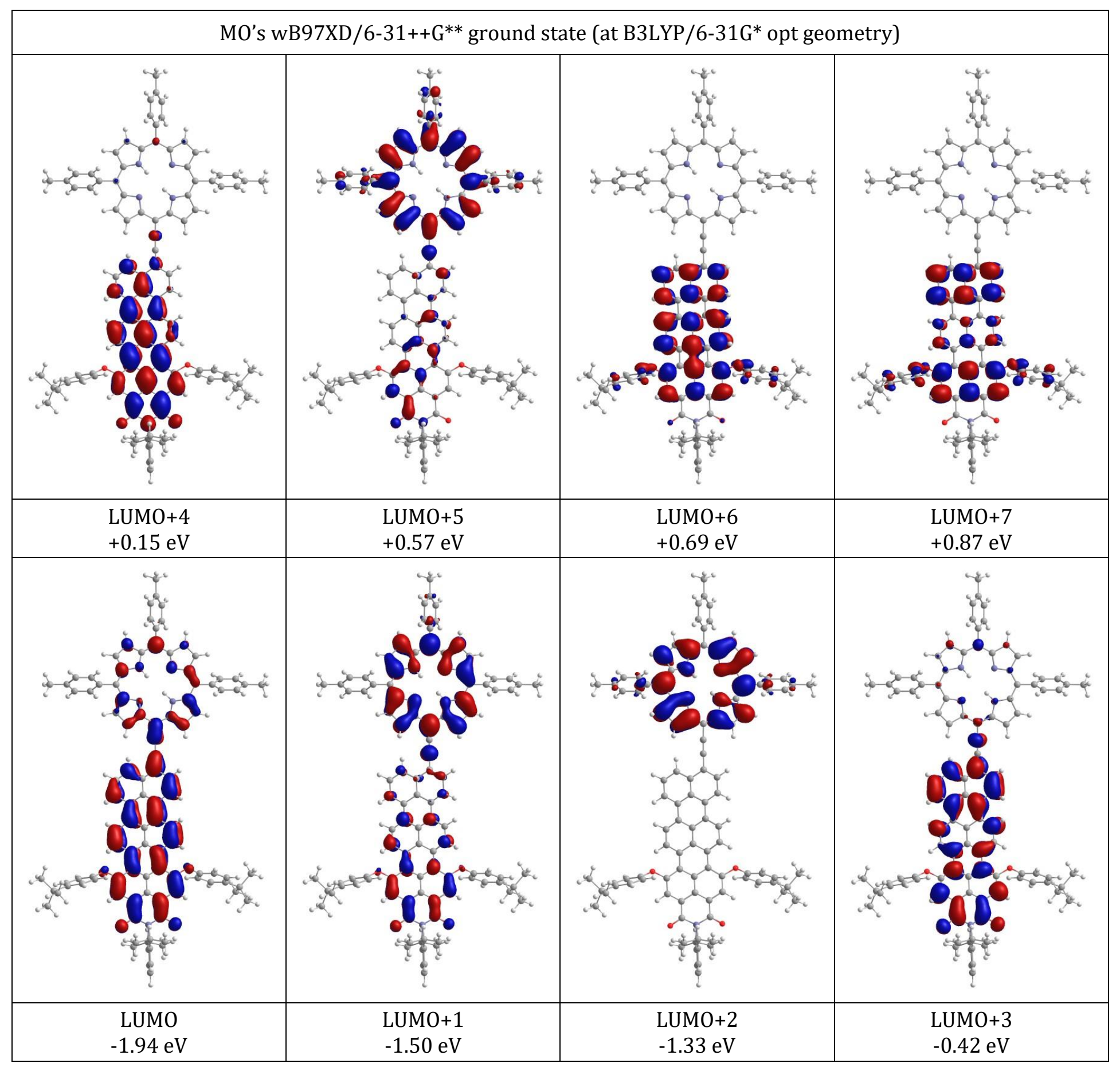

Figure S42. Empty MOs for P-TMI in toluene from DFT calculations. 


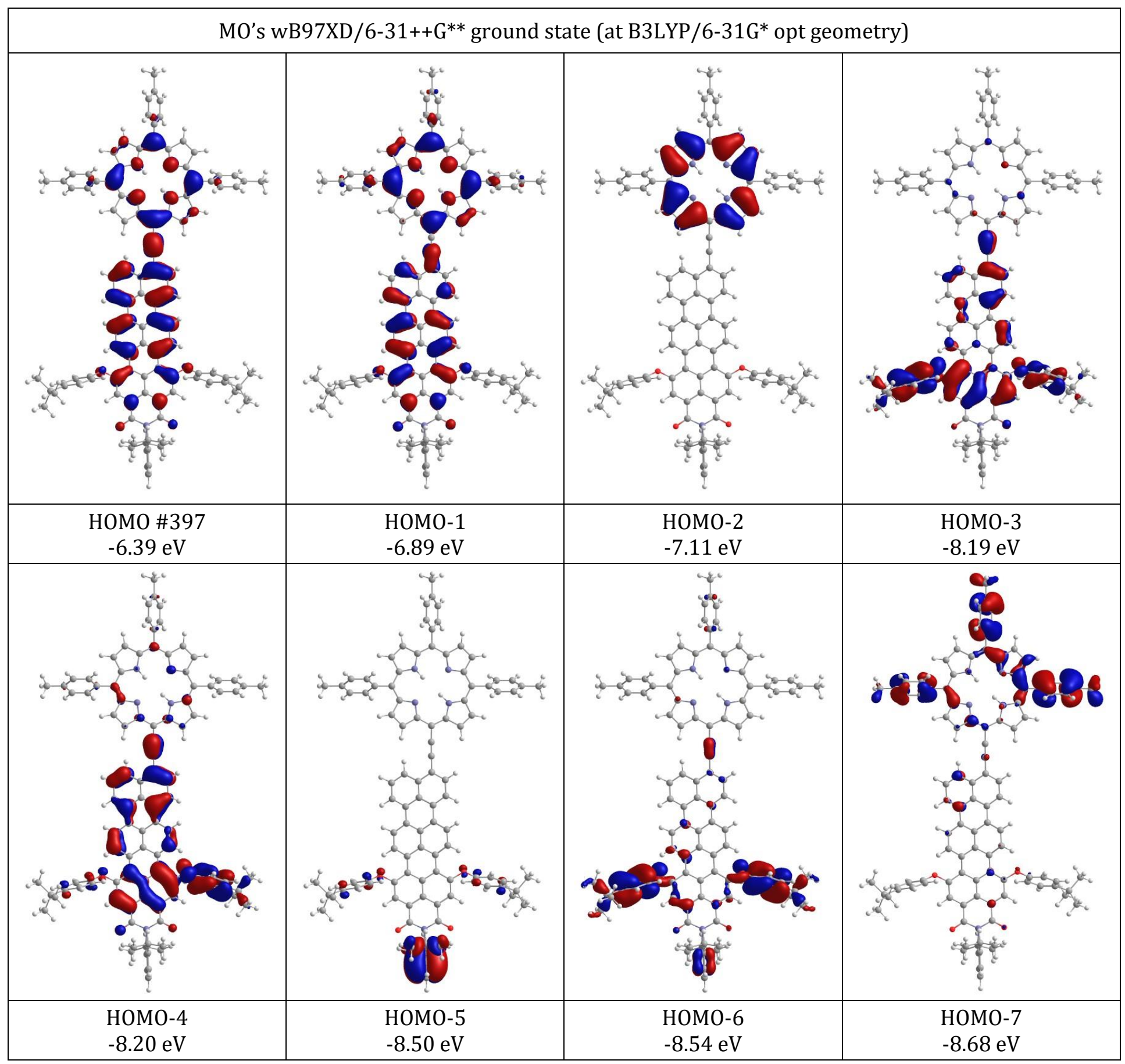

Figure S43. Filled MOs for P-TMI in toluene from DFT calculations. 


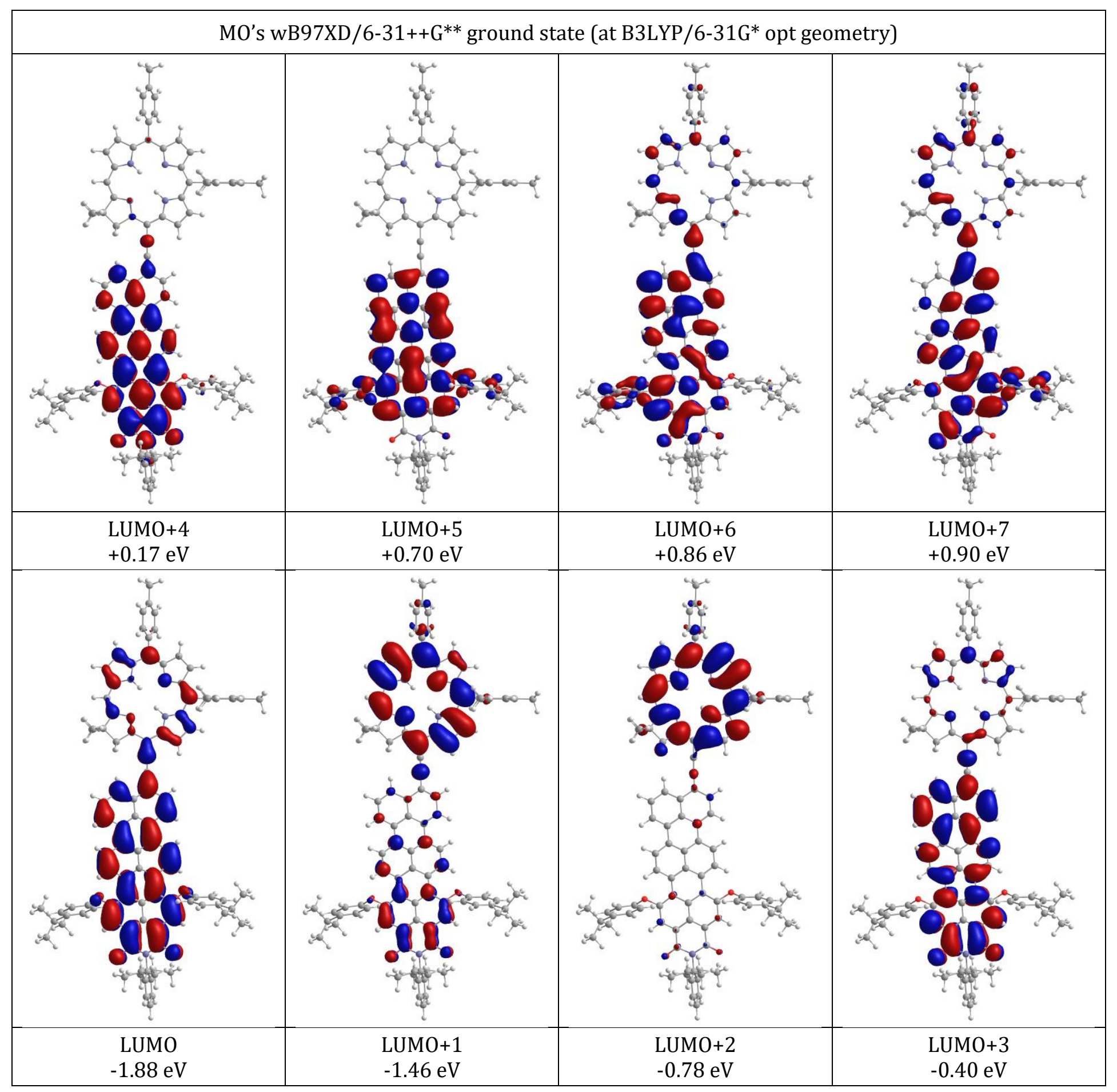

Figure S44. Empty MOs for C-TMI in toluene from DFT calculations. 


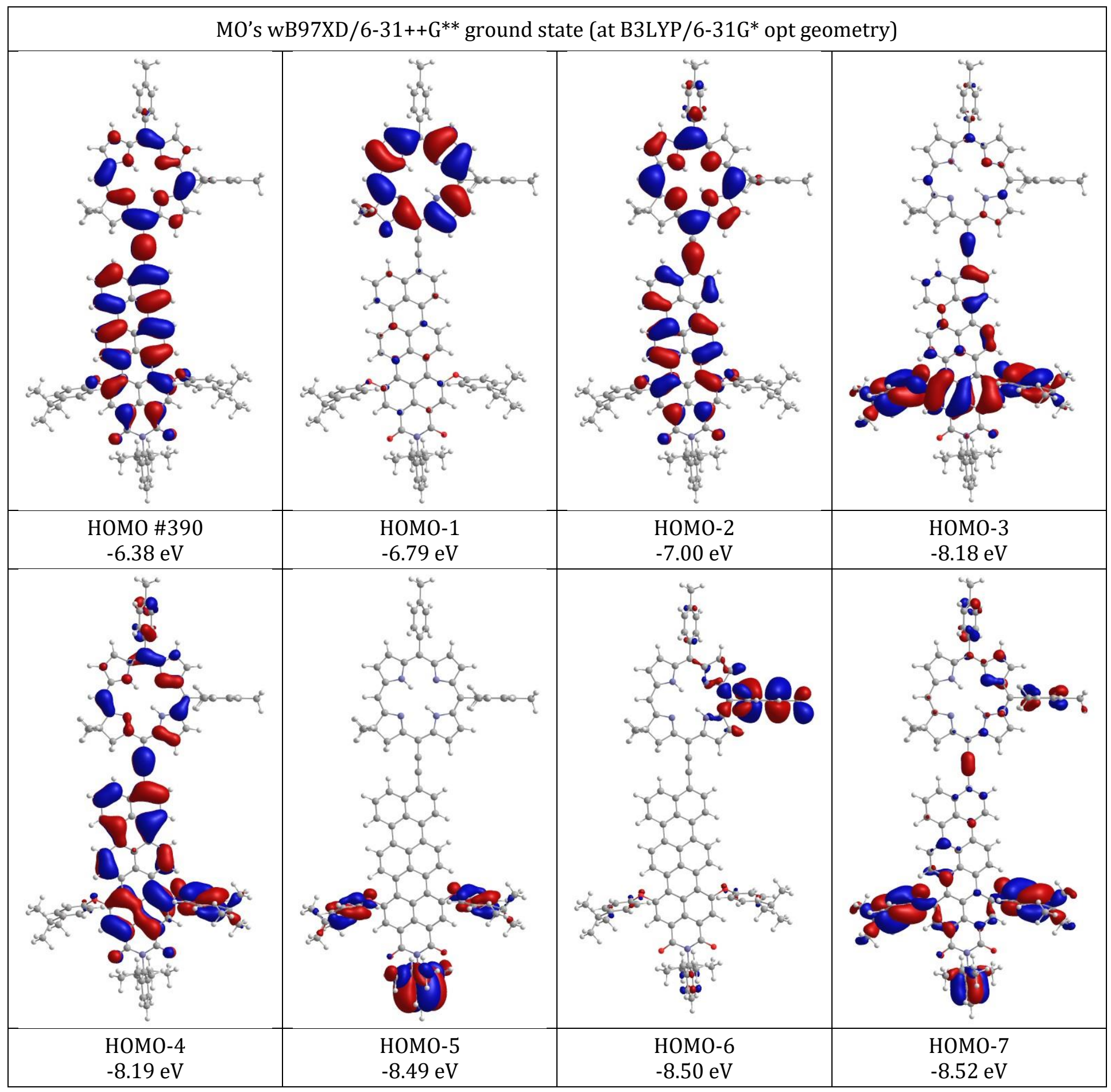

Figure S45. Filled MOs for C-TMI in toluene from DFT calculations. 


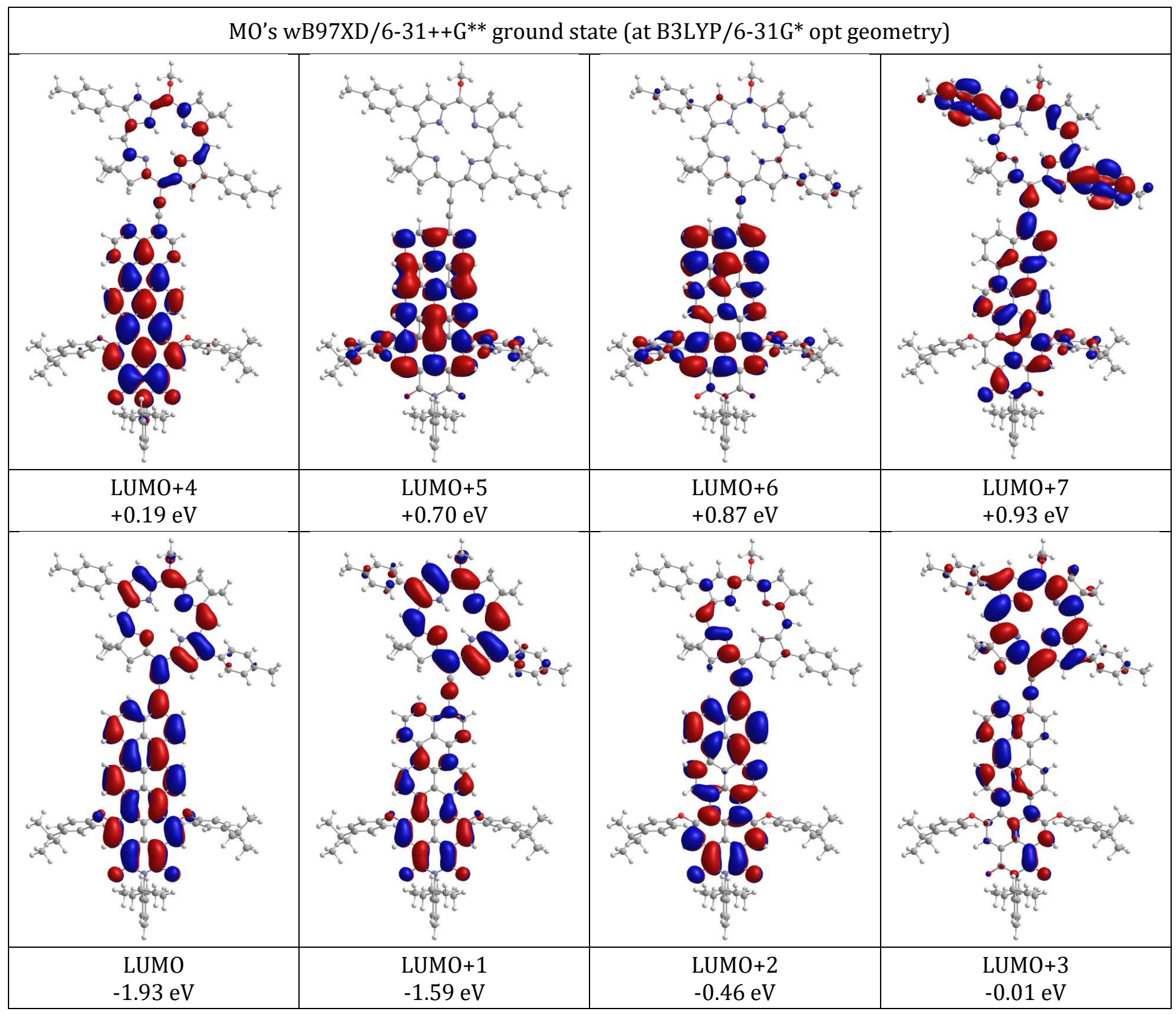

Figure S46. Empty MOs for B-TMI in toluene from DFT calculations. 


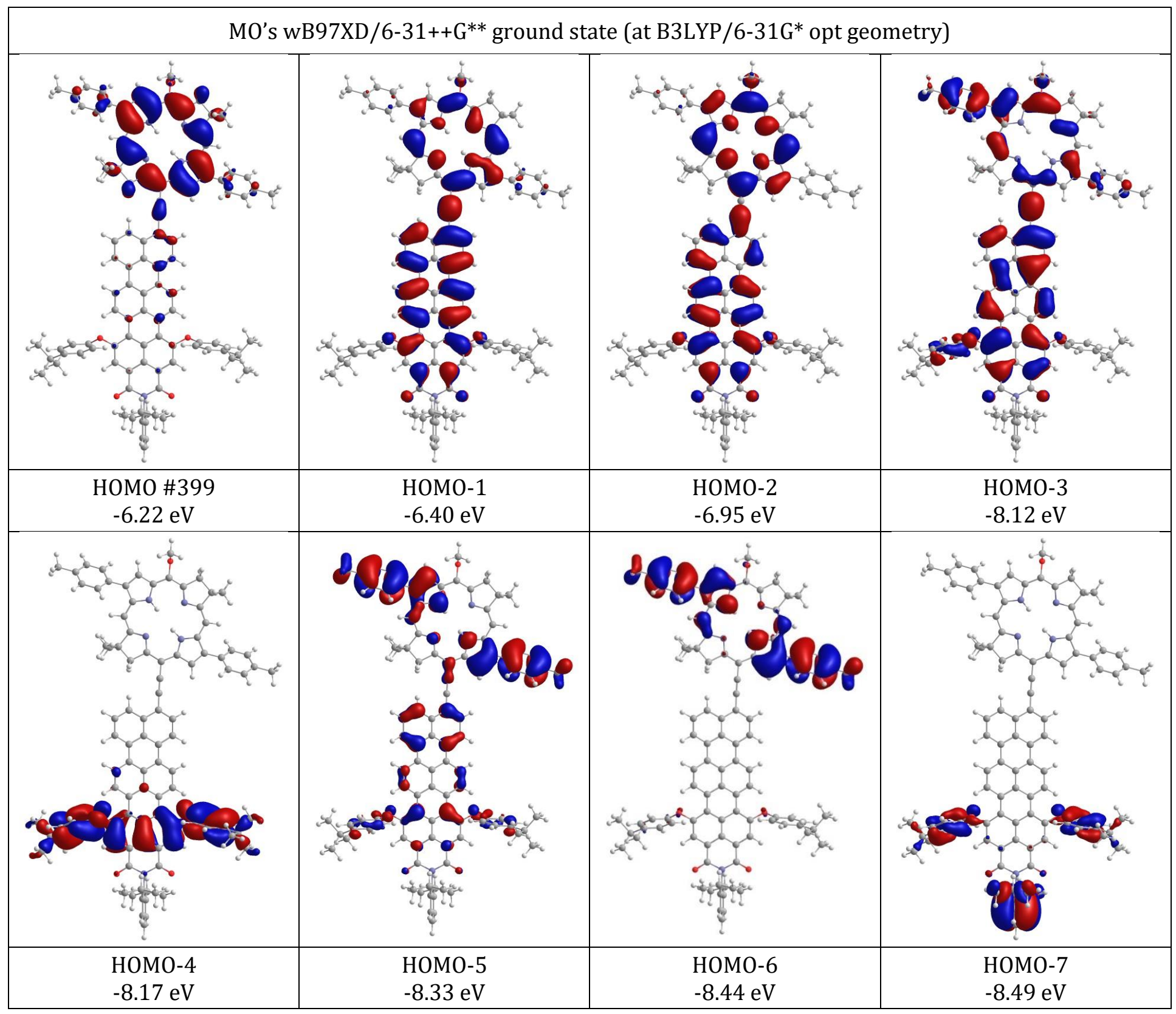

Figure S47. Filled MOs for B-TMI in toluene from DFT calculations. 


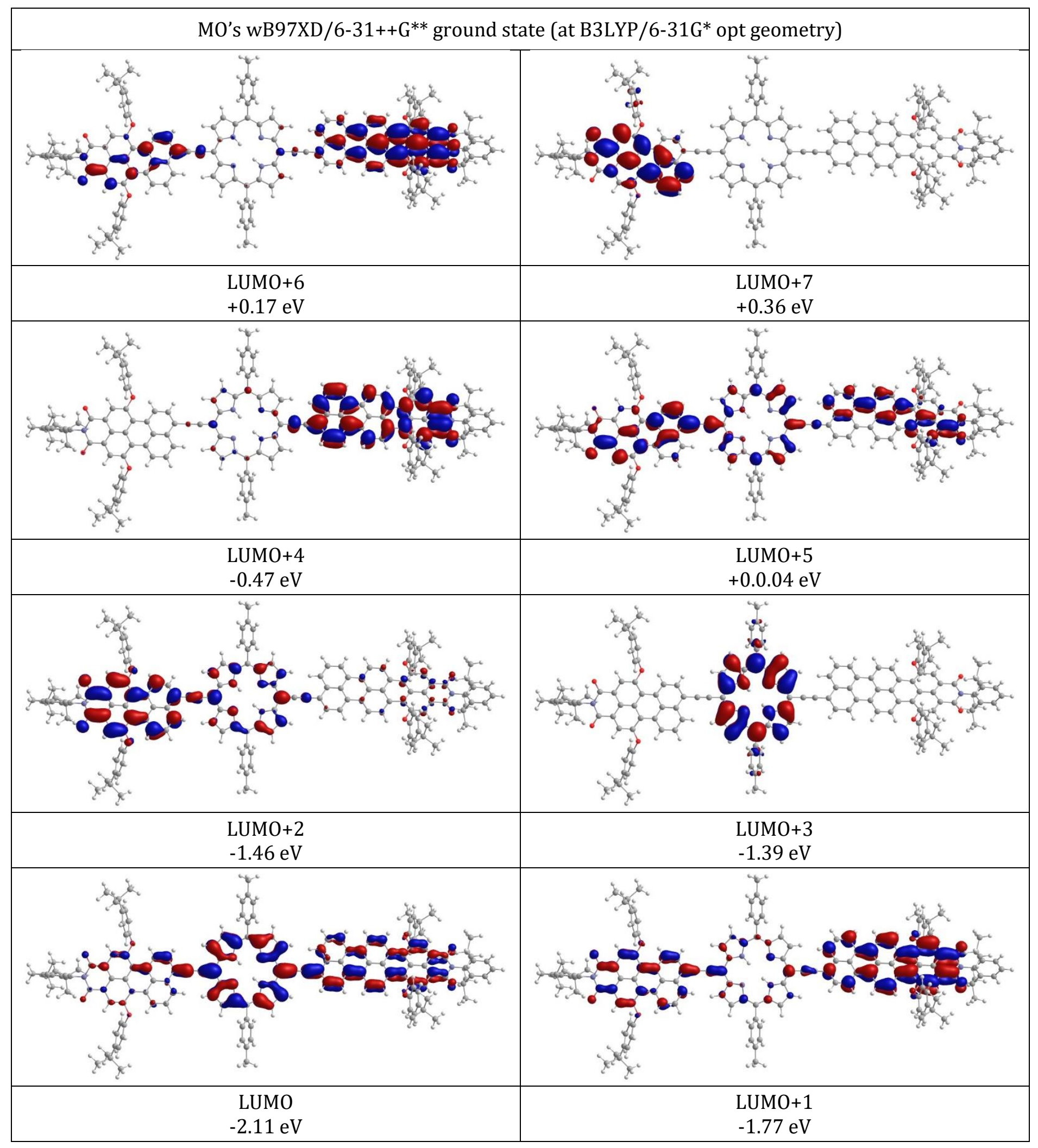

Figure S48. Empty MOs for PMI-P-TMI in toluene from DFT calculations. 


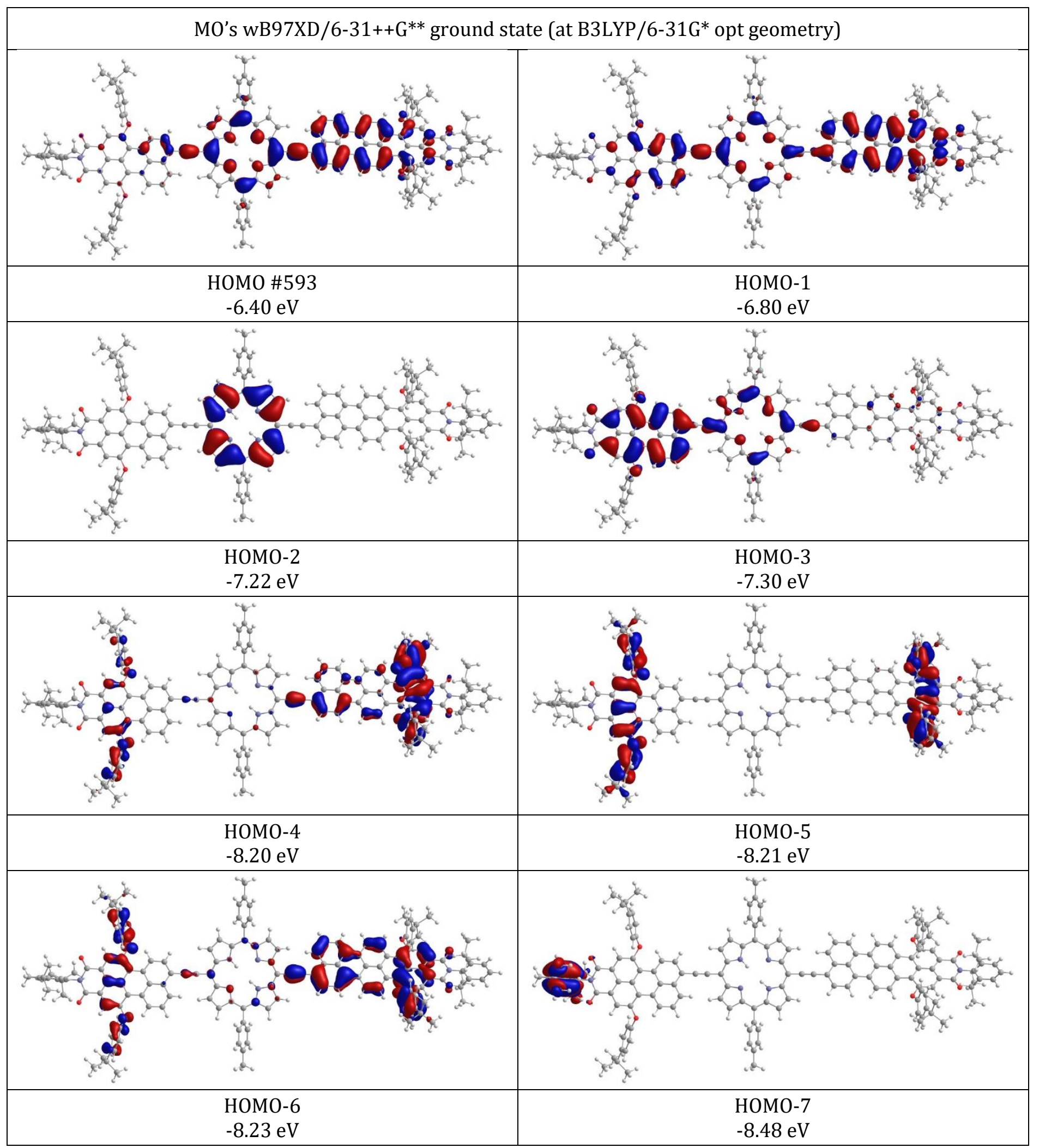

Figure S49. Filled MOs for PMI-P-TMI in toluene from DFT calculations. 


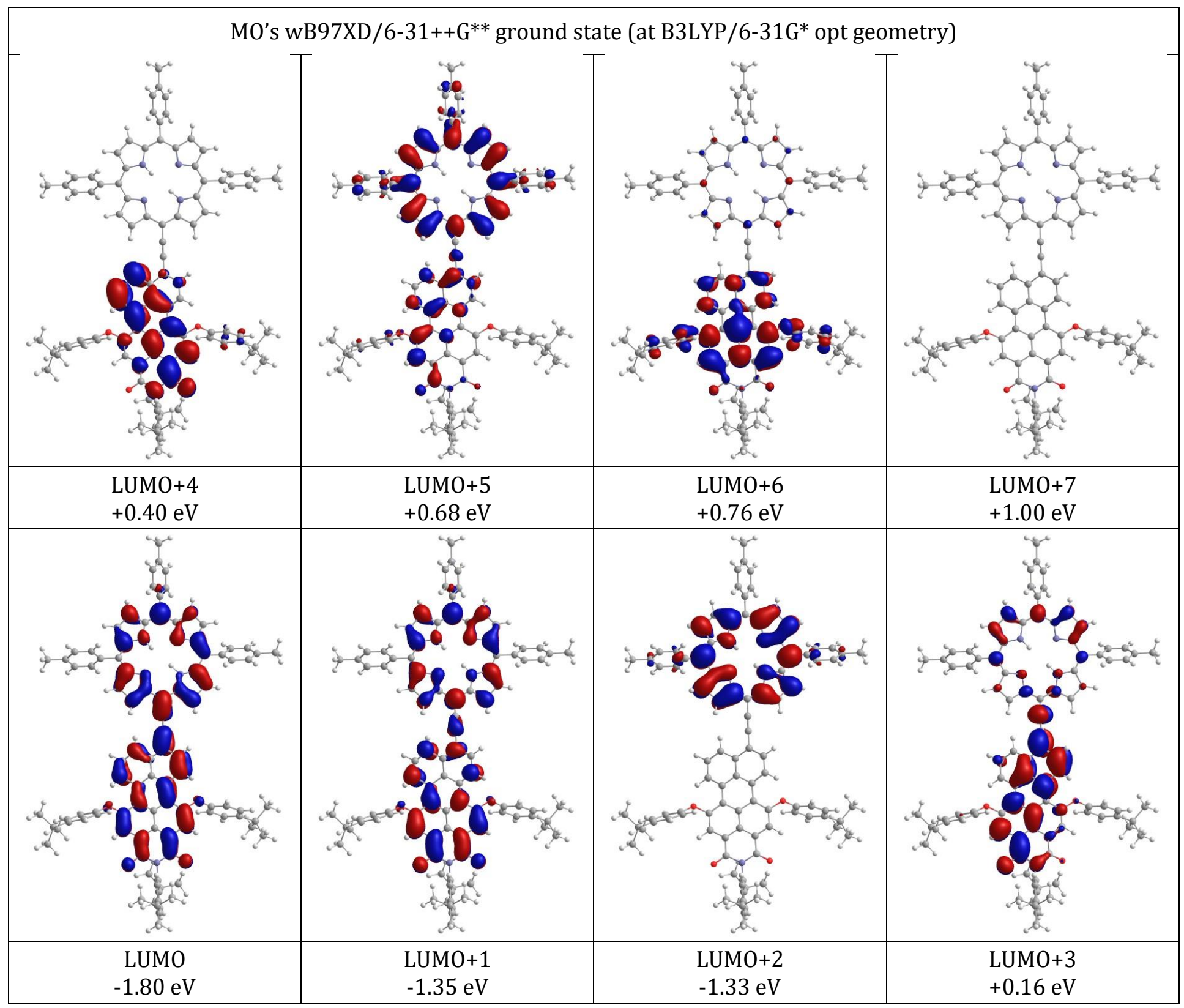

Figure S50. Empty MOs for P-PMI in toluene from DFT calculations. 


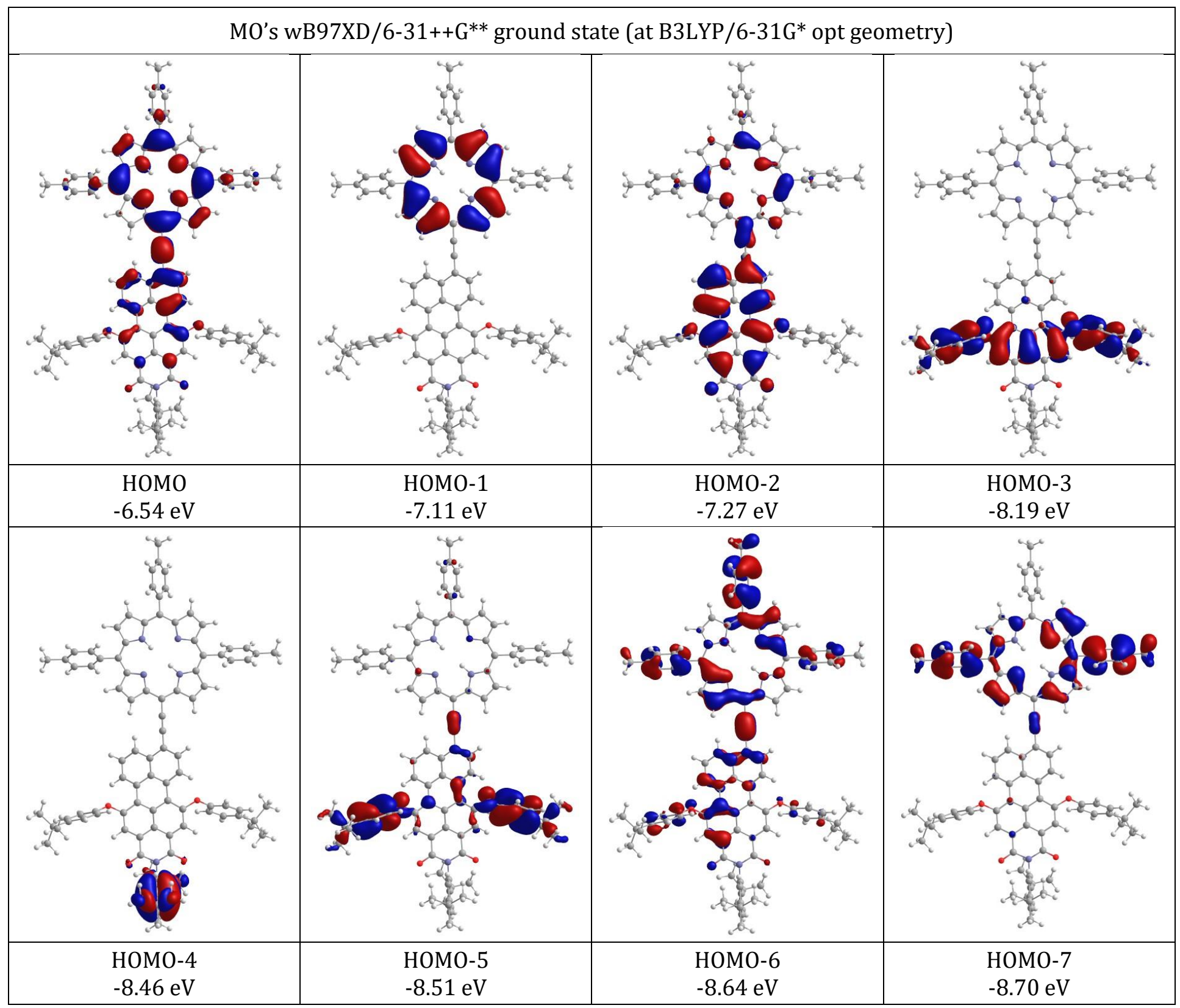

Figure S51. Filled MOs for P-PMI in toluene from DFT calculations. 


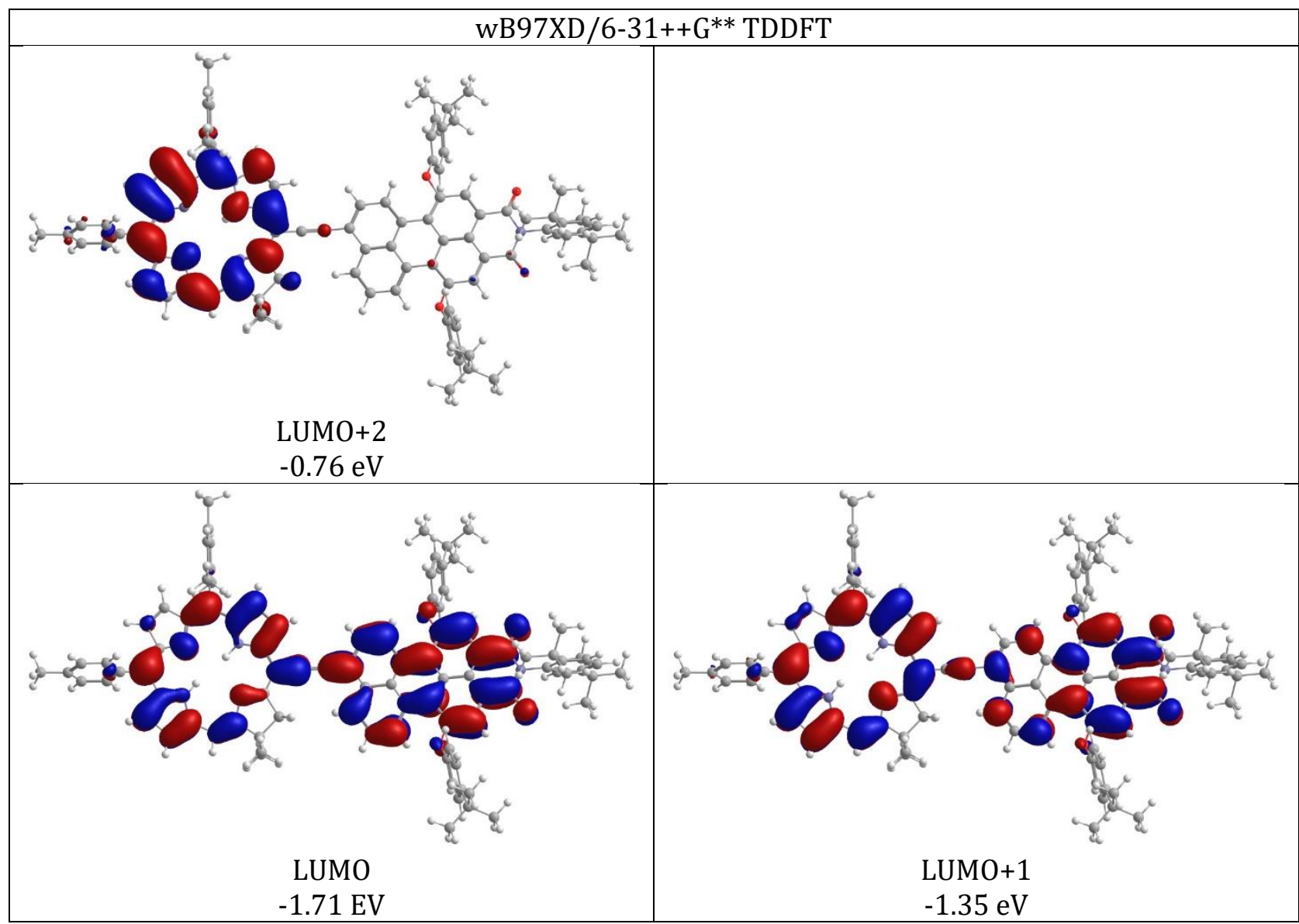

Figure S52. Empty MOs for C-PMI in toluene from DFT calculations. 


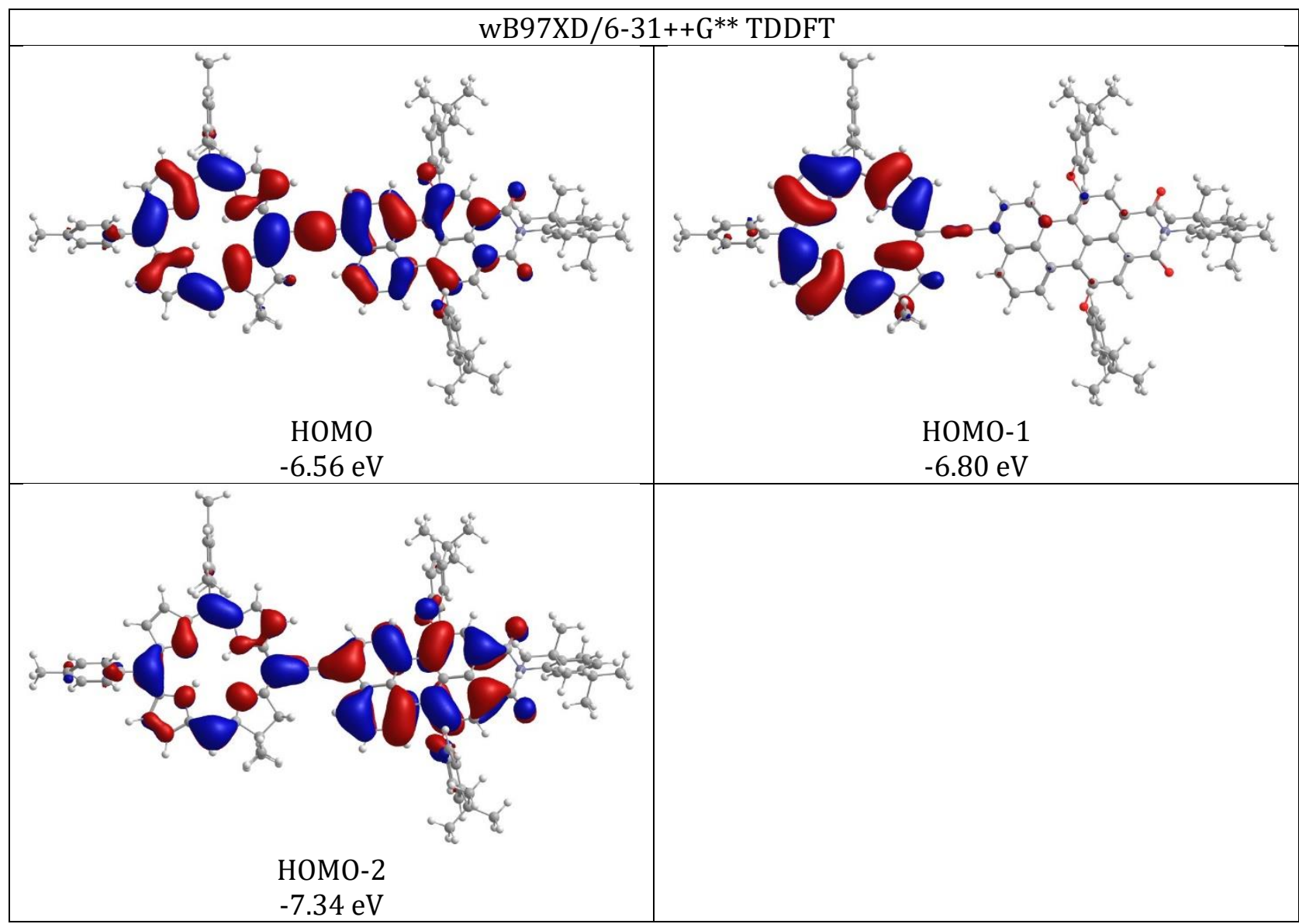

Figure S53. Filled MOs for C-PMI in toluene from DFT calculations. 


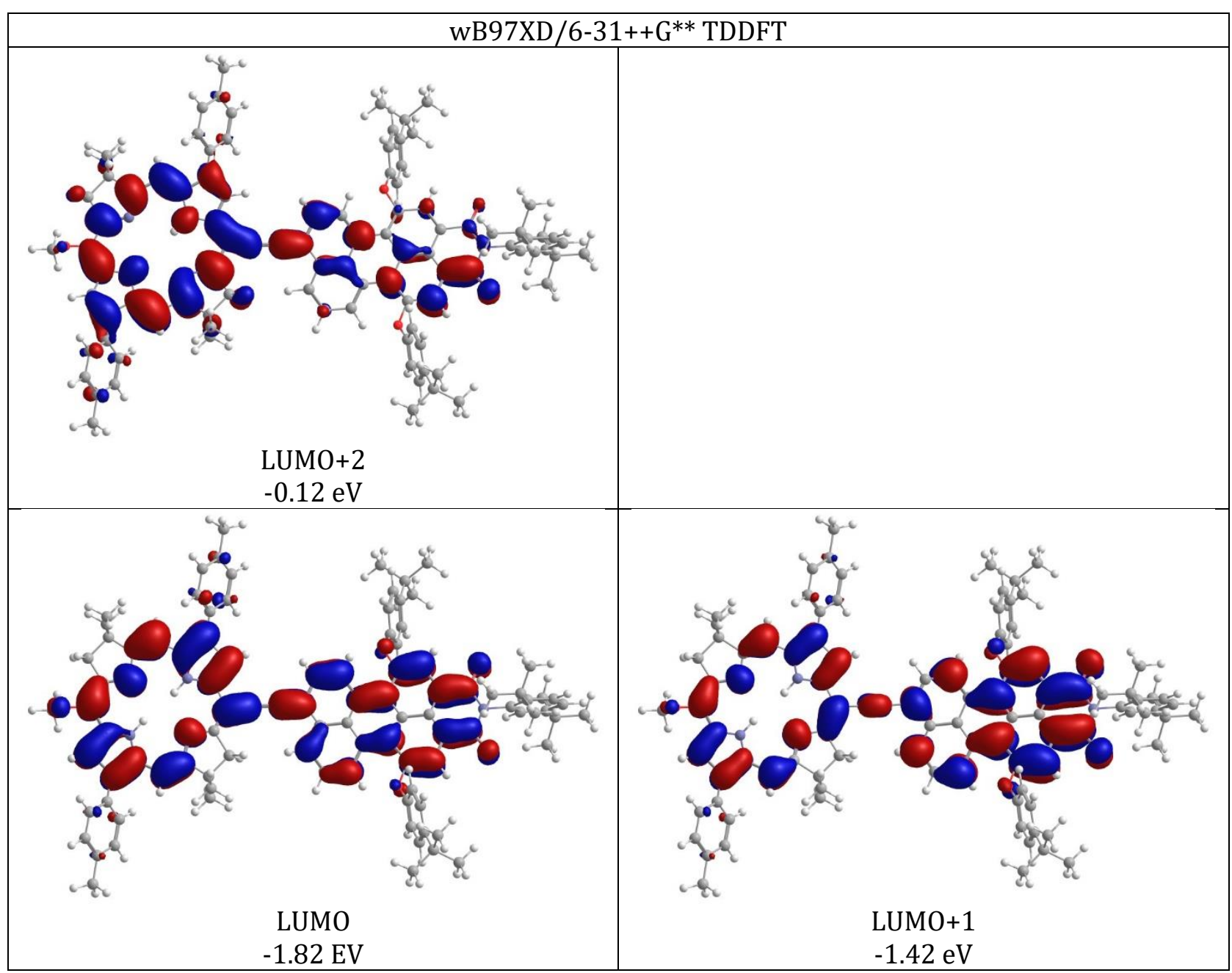

Figure S54. Empty MOs for B-PMI in toluene from DFT calculations. 


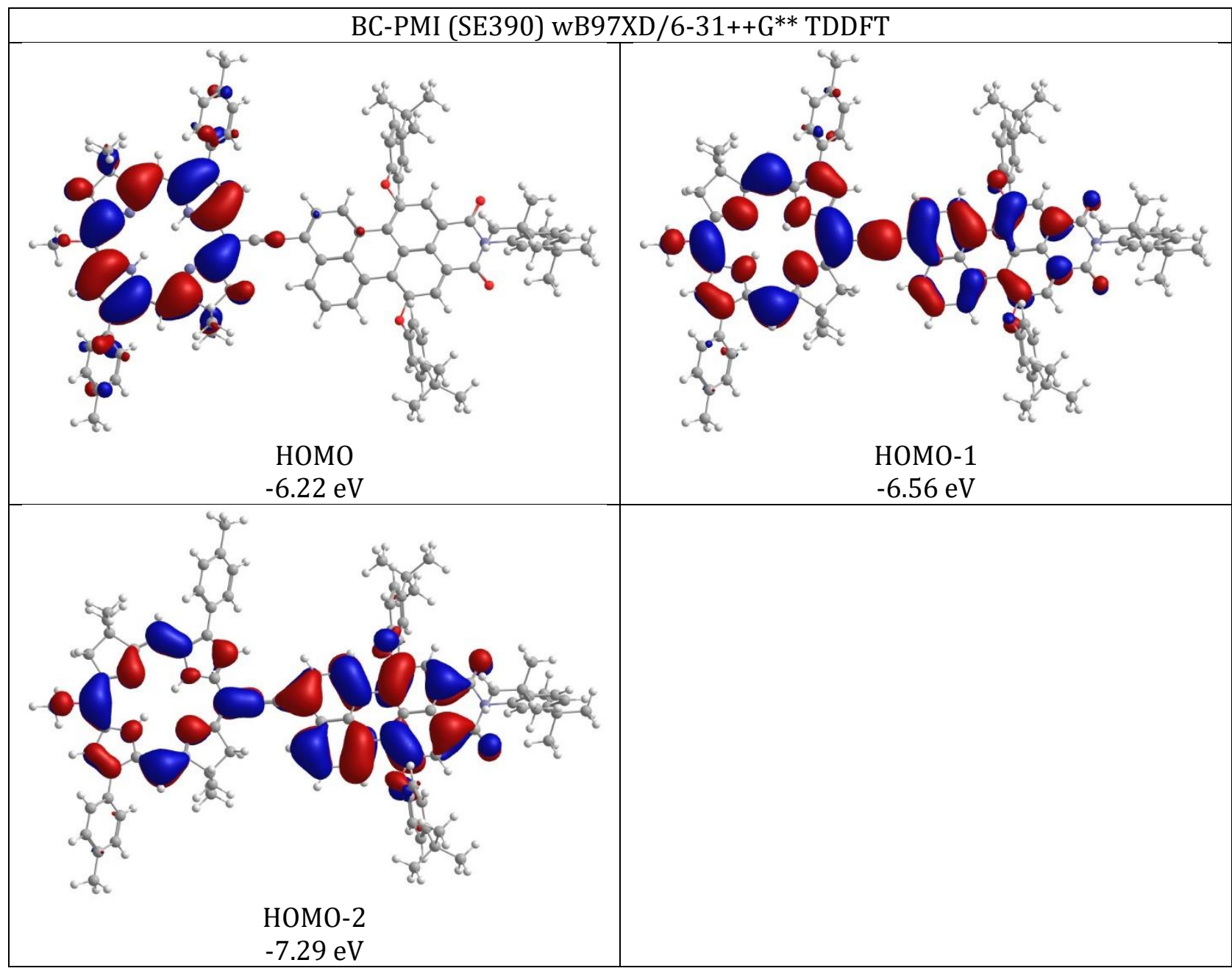

Figure S55. Filled MOs for B-PMI in toluene from DFT calculations. 


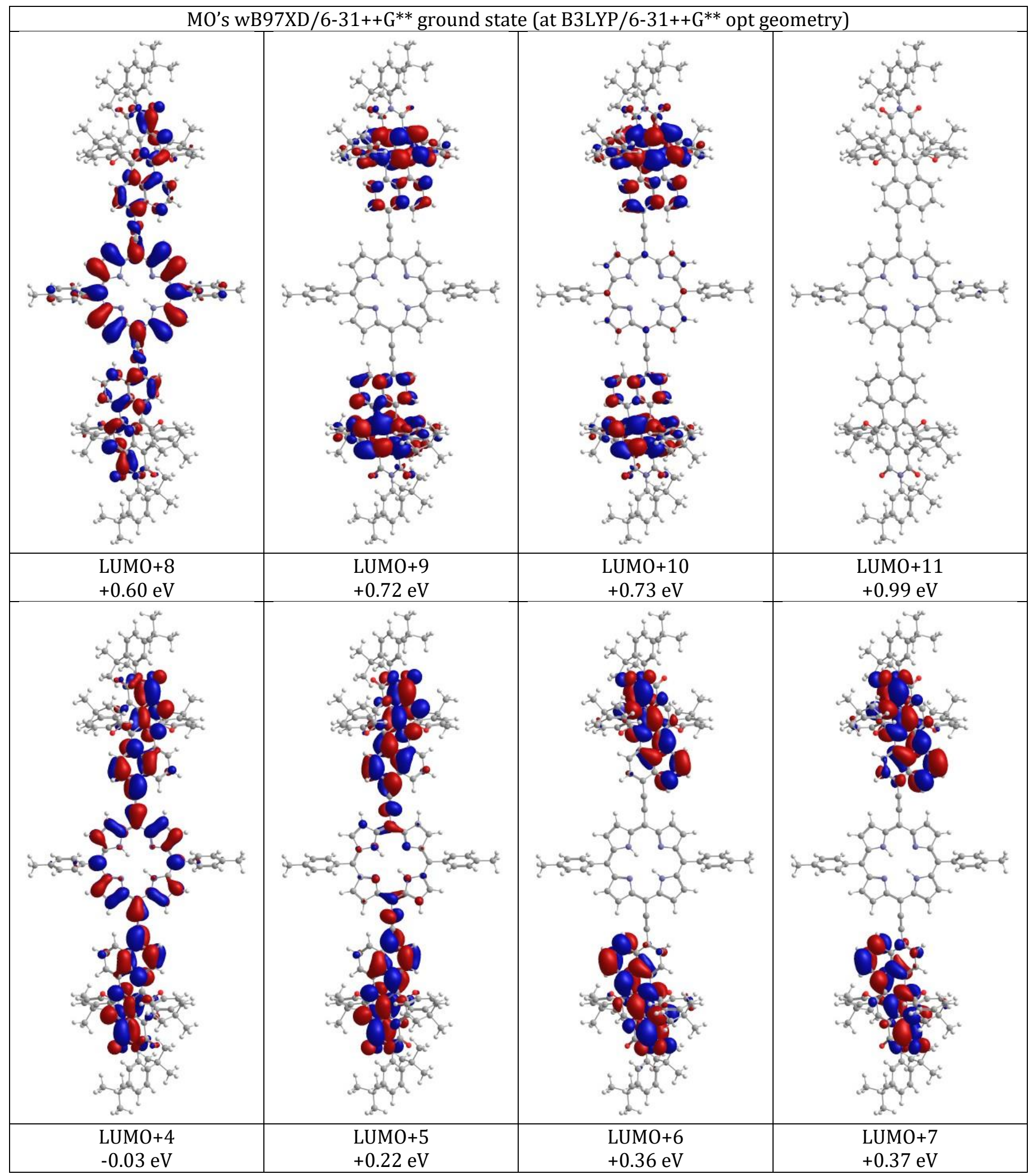




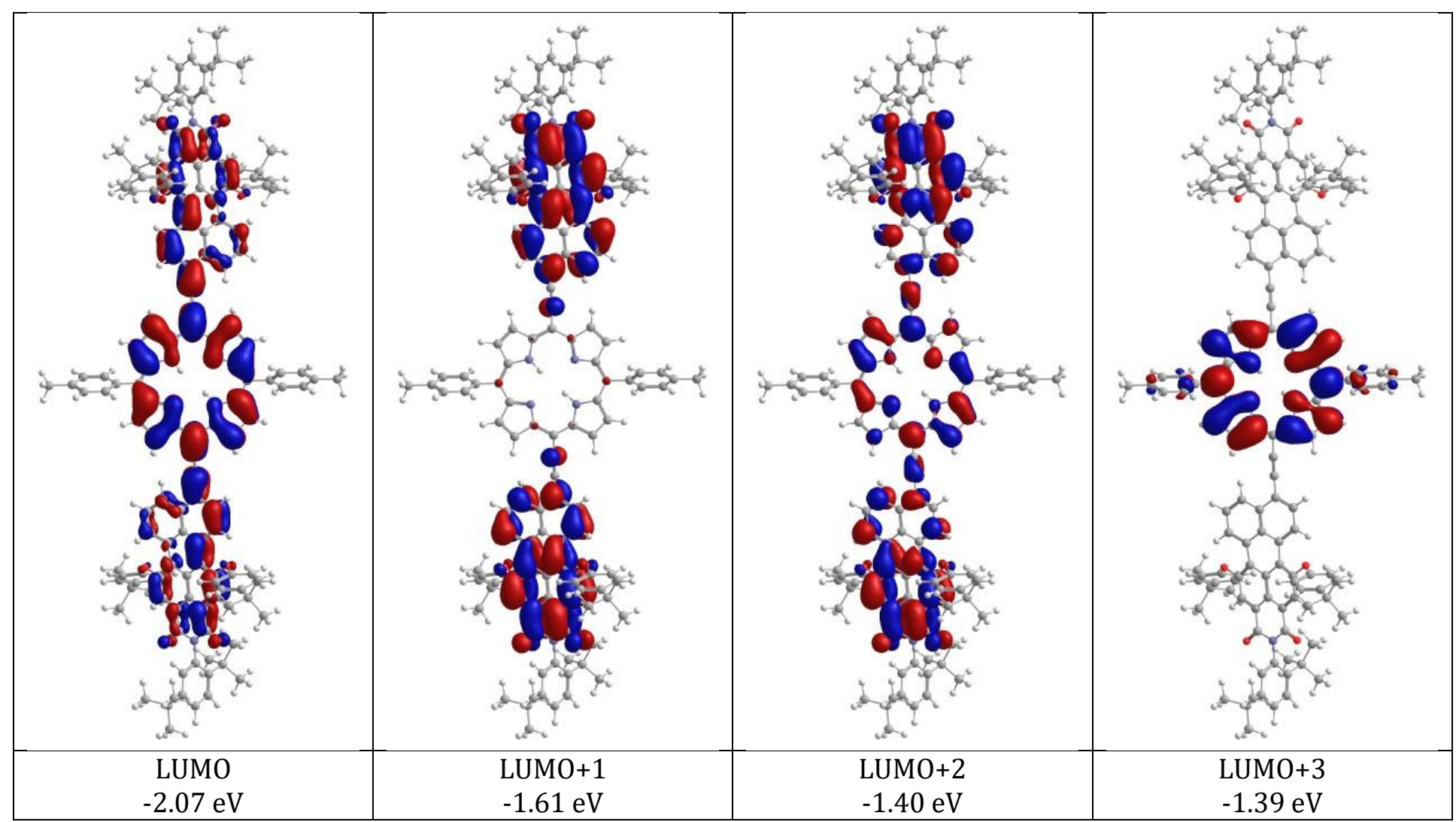

Figure S56. Empty MOs for PMI-P-PMI in toluene from DFT calculations. 


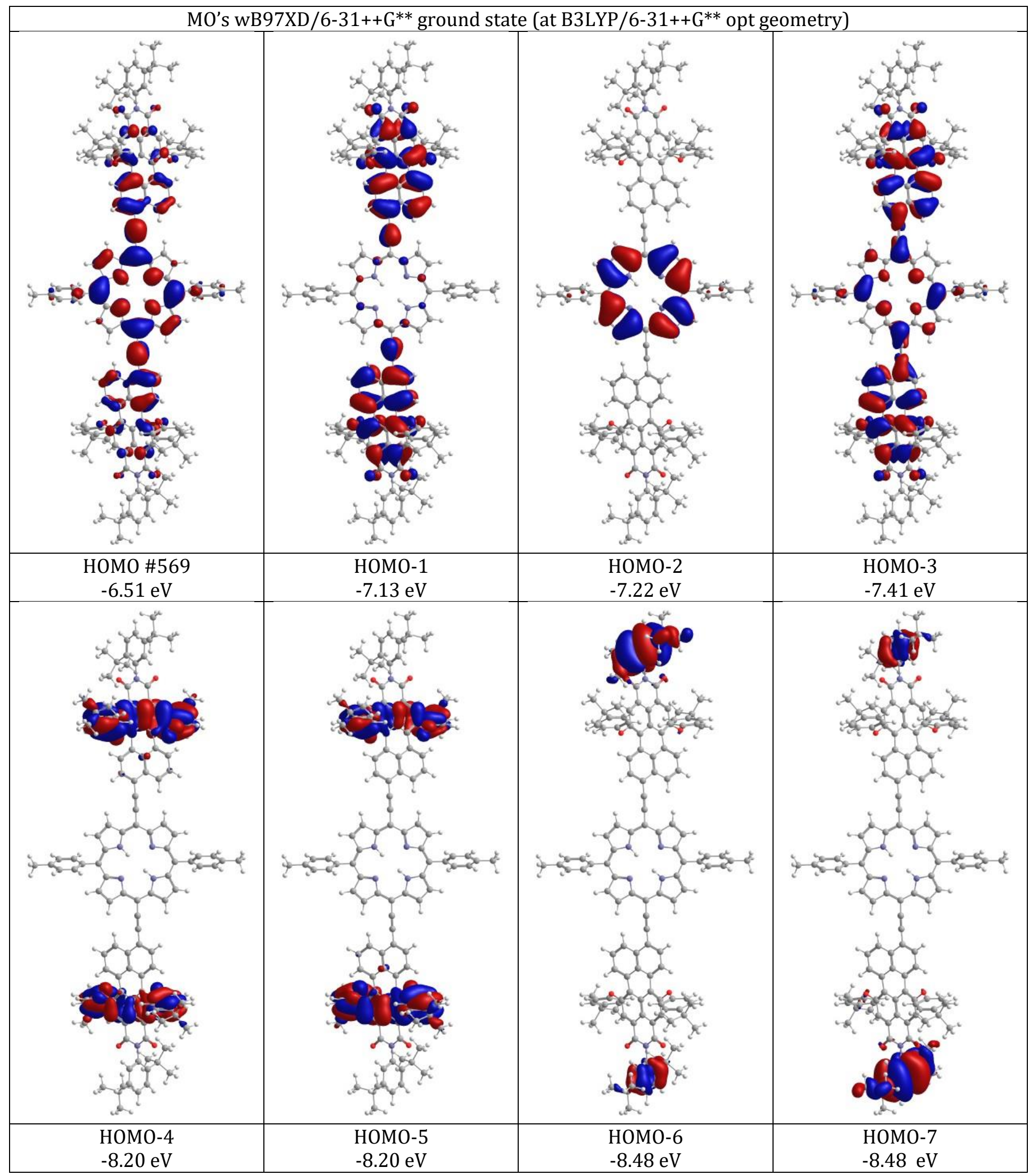




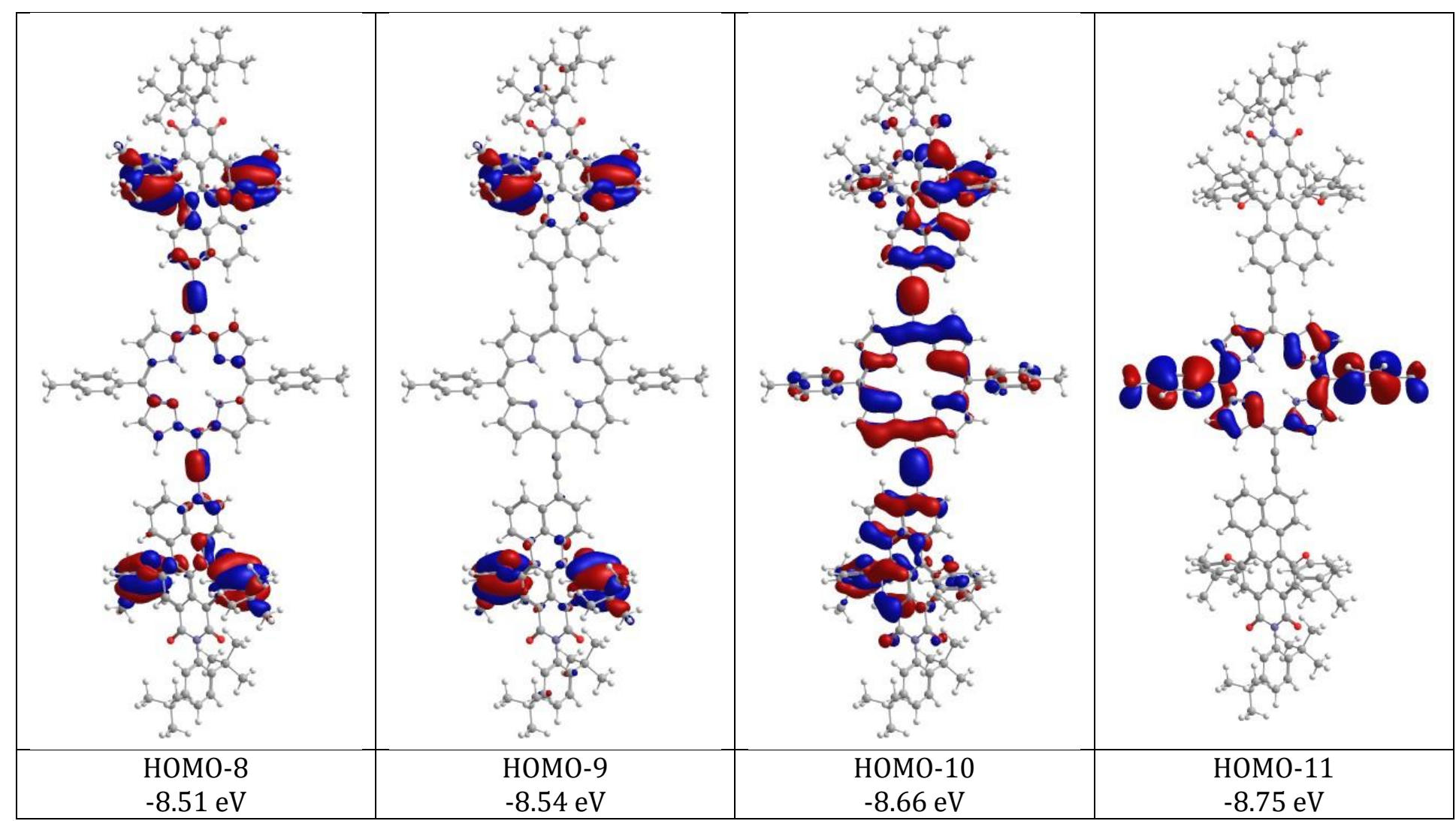

Figure S57. Filled MOs for PMI-P-PMI in toluene from DFT calculations. 
Section S5

Molecular Orbital

Correlation

Diagrams 


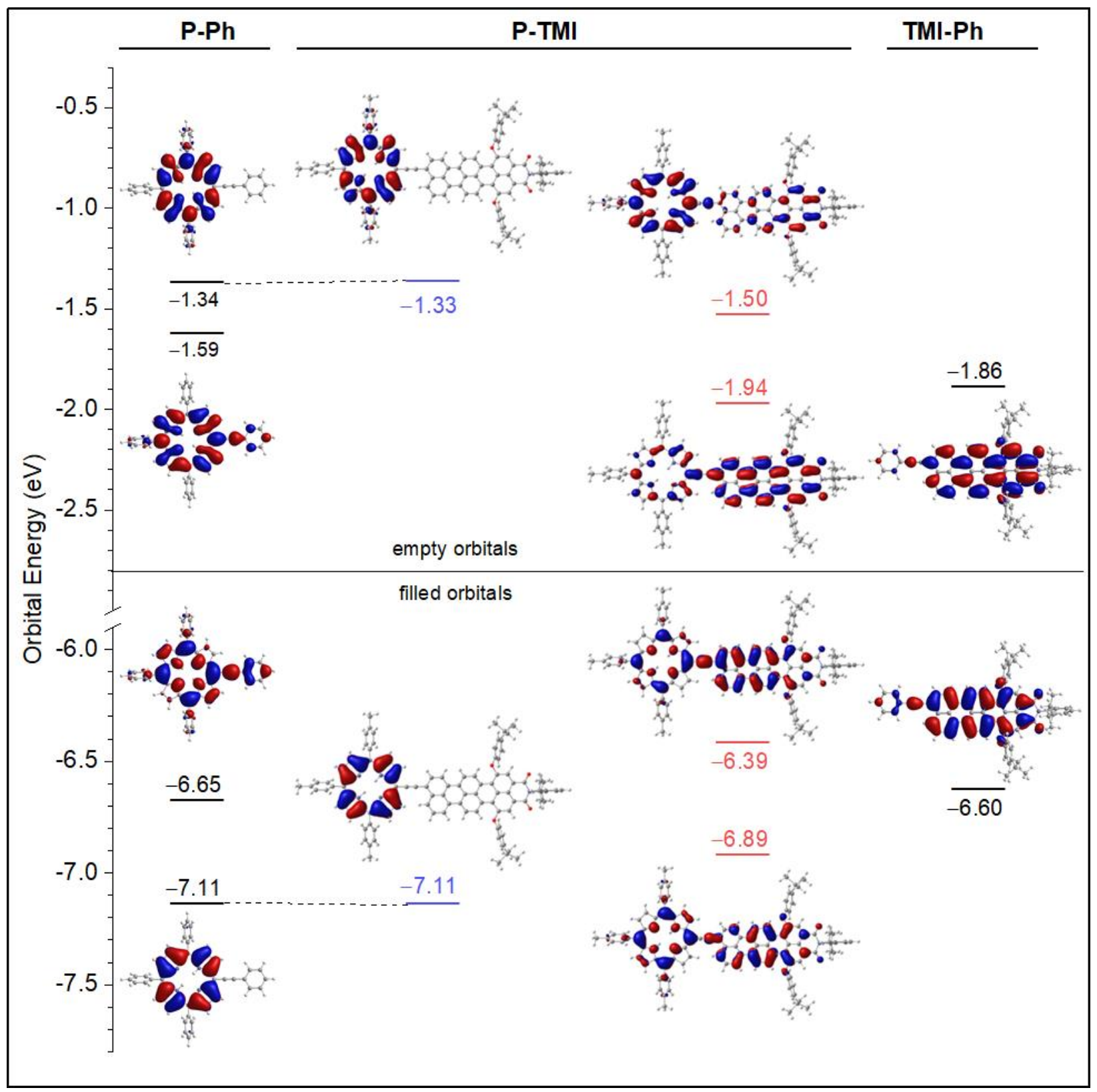

Figure S58. MO correlation diagram for dyad P-TMI, porphyrin benchmark P-Ph, and terrylene benchmark TMI-Ph. The MOs for the dyad with energies indicated with blue font are essentially porphyrin MOs (nodes at the linker attachment site); those with energies indicated in red font are mixtures of the terrylene and porphyrin orbitals. This is a duplicate of Figure 7 of the paper and is included here for comparison with the analogous spectra for the other dyads. 


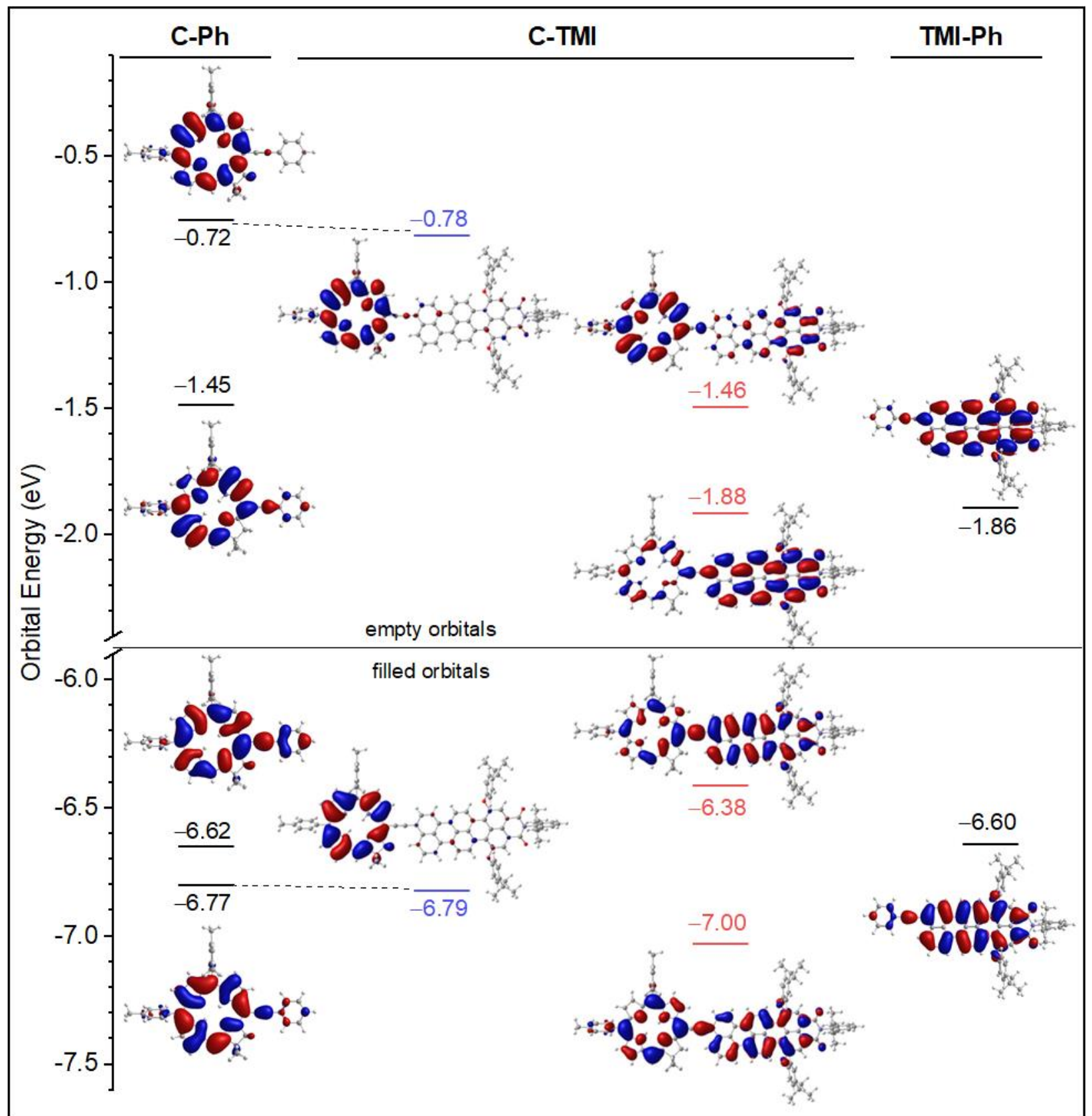

Figure S59. MO correlation diagram for dyad C-TMI, chlorin benchmark C-Ph, and terrylene benchmark TMI-Ph. The MOs for the dyad with energies indicated with blue font are essentially chlorin MOs (nodes at the linker attachment site); those with energies indicated in red font are mixtures of the terrylene and porphyrin orbitals. 


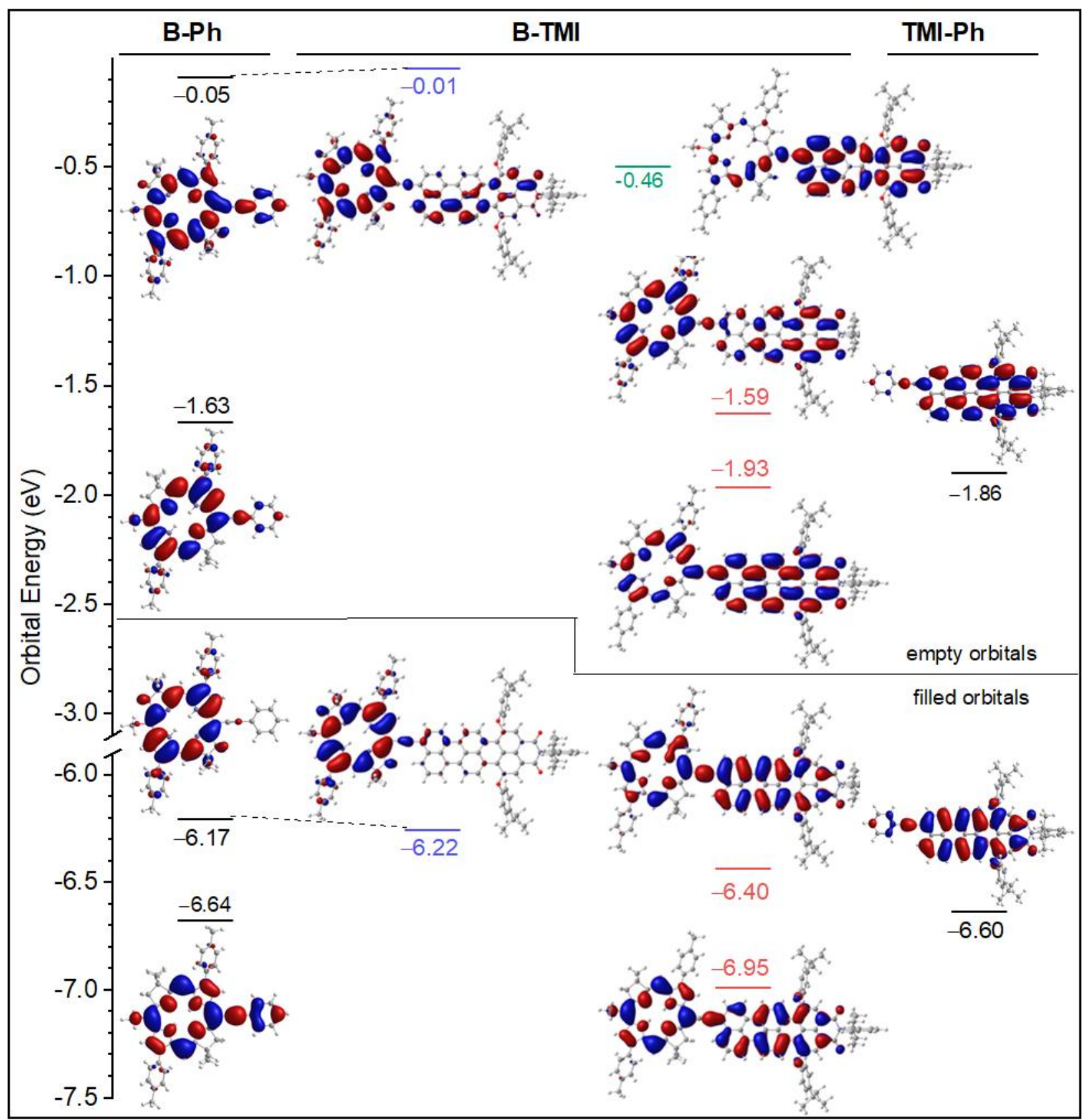

Figure S60. MO correlation diagram for dyad B-TMI, bacteriochlorin benchmark B-Ph, and terrylene benchmark TMI-Ph. The MOs for the dyad with energies indicated with blue font are essentially bacteriochlorin MOs (nodes at the linker attachment site); those with energies indicated in red font are mixtures of the terrylene and porphyrin orbitals; the one indicated in green is largely a terrylene orbital with some bacteriochlorin character. 


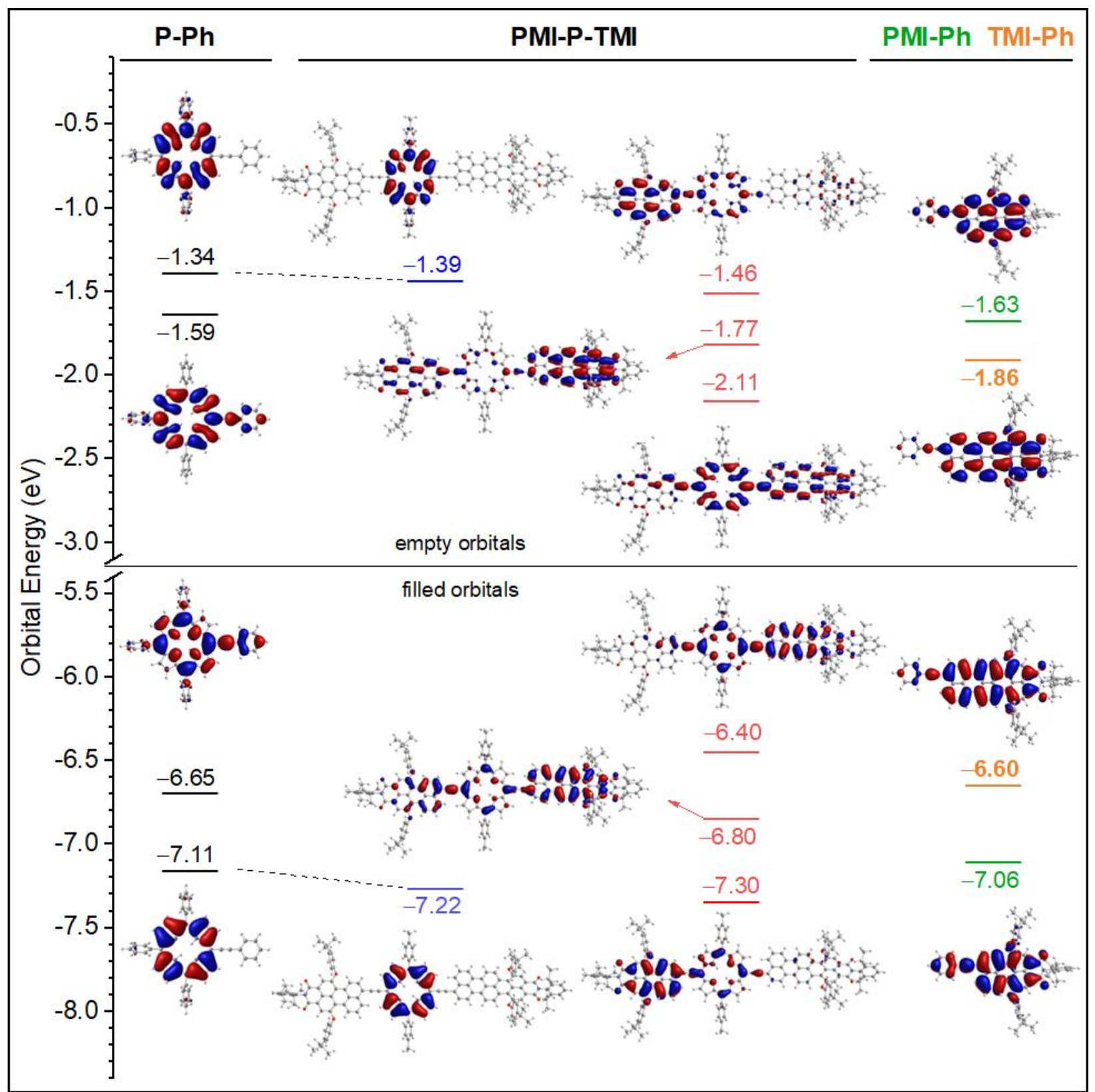

Figure S61. MO correlation diagram for triad PMI-P-TMI, porphyrin benchmark P-Ph, terrylene benchmark TMI-Ph and perylene benchmark PMI-Ph. The MOs for the triad with energies indicated with blue font are essentially porphyrin MOs (nodes at the linker attachment site); those with energies indicated in red font are mixtures (effectively bonding, nonbonding and antibonding trios) of the terrylene, perylene and porphyrin orbitals. This is a duplicate of Figure 8 of the paper and is included here for comparison with the analogous spectra for triad PMI-P-PMI. 


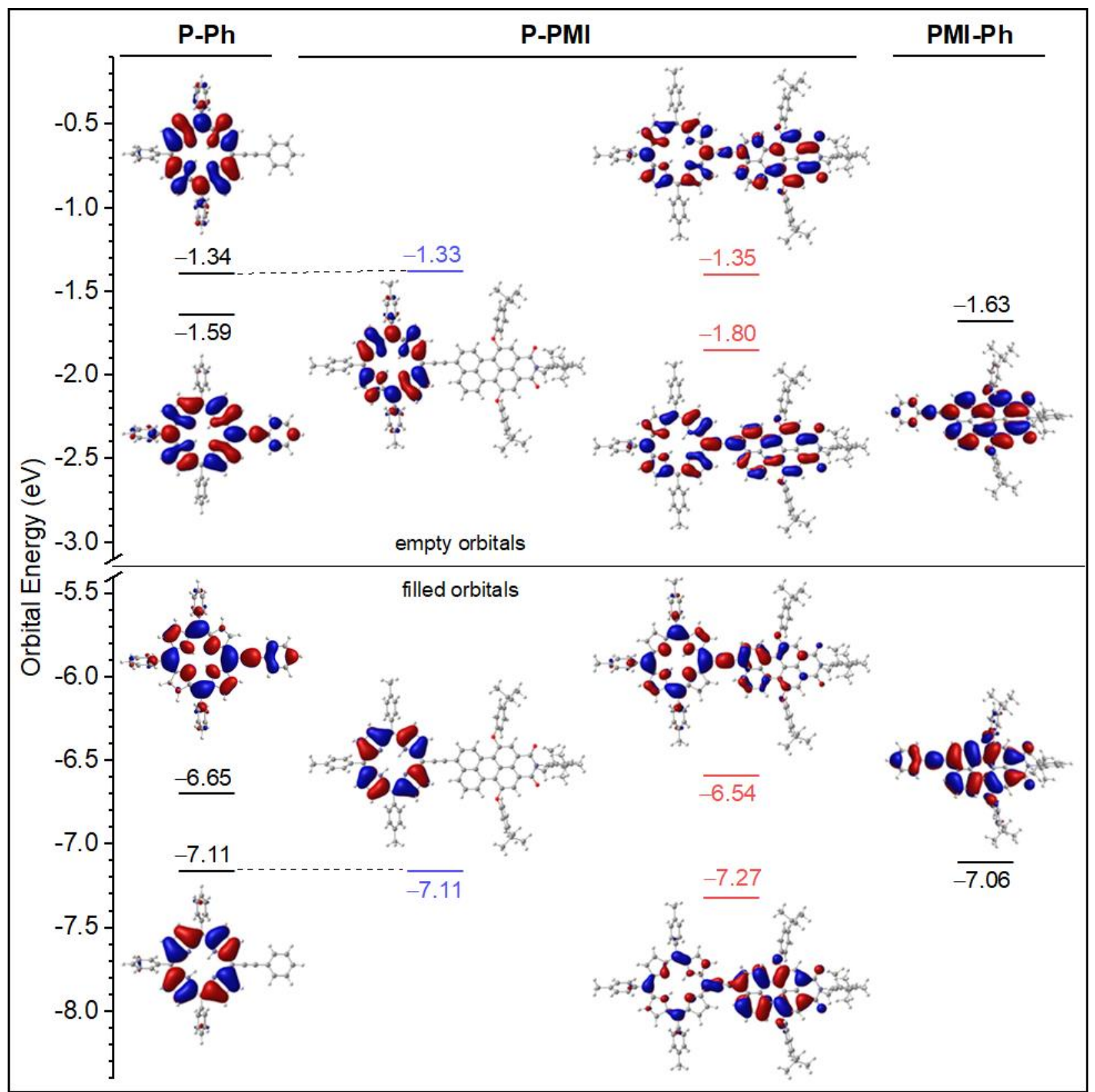

Figure S62. MO correlation diagram for dyad P-PMI, porphyrin benchmark P-Ph, and perylene benchmark PMI-Ph. The MOs for the dyad with energies indicated with blue font are essentially porphyrin MOs (nodes at the linker attachment site); those with energies indicated in red font are mixtures of the perylene and porphyrin orbitals. 


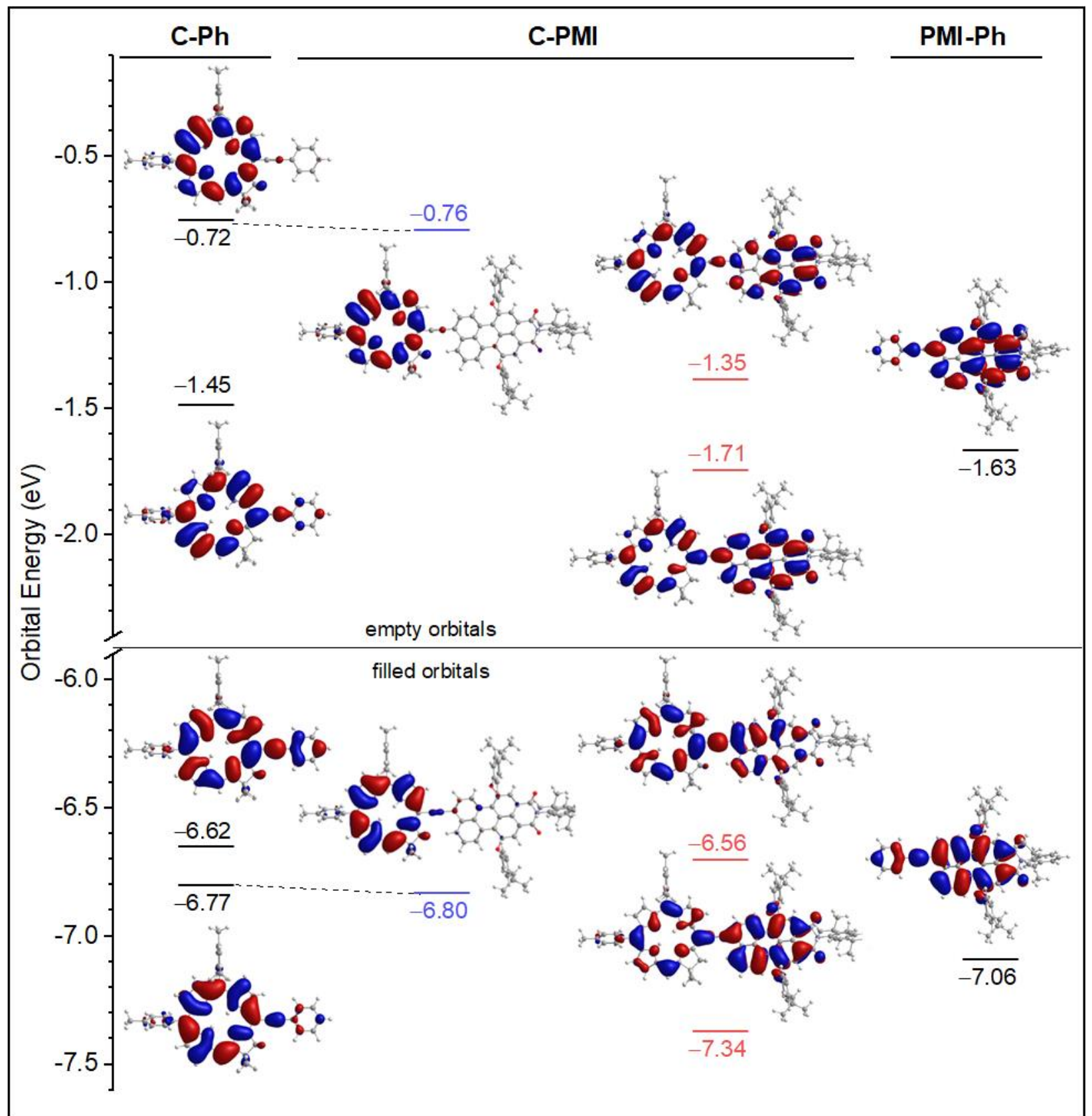

Figure S63. MO correlation diagram for dyad C-PMI, chlorin benchmark C-Ph and perylene benchmark PMI-Ph. The MOs for the dyad with energies indicated with blue font are essentially chlorin MOs; the MOs of the dyad with energies indicated with red font are mixtures of the corresponding orbitals of the perylene and chlorin. 


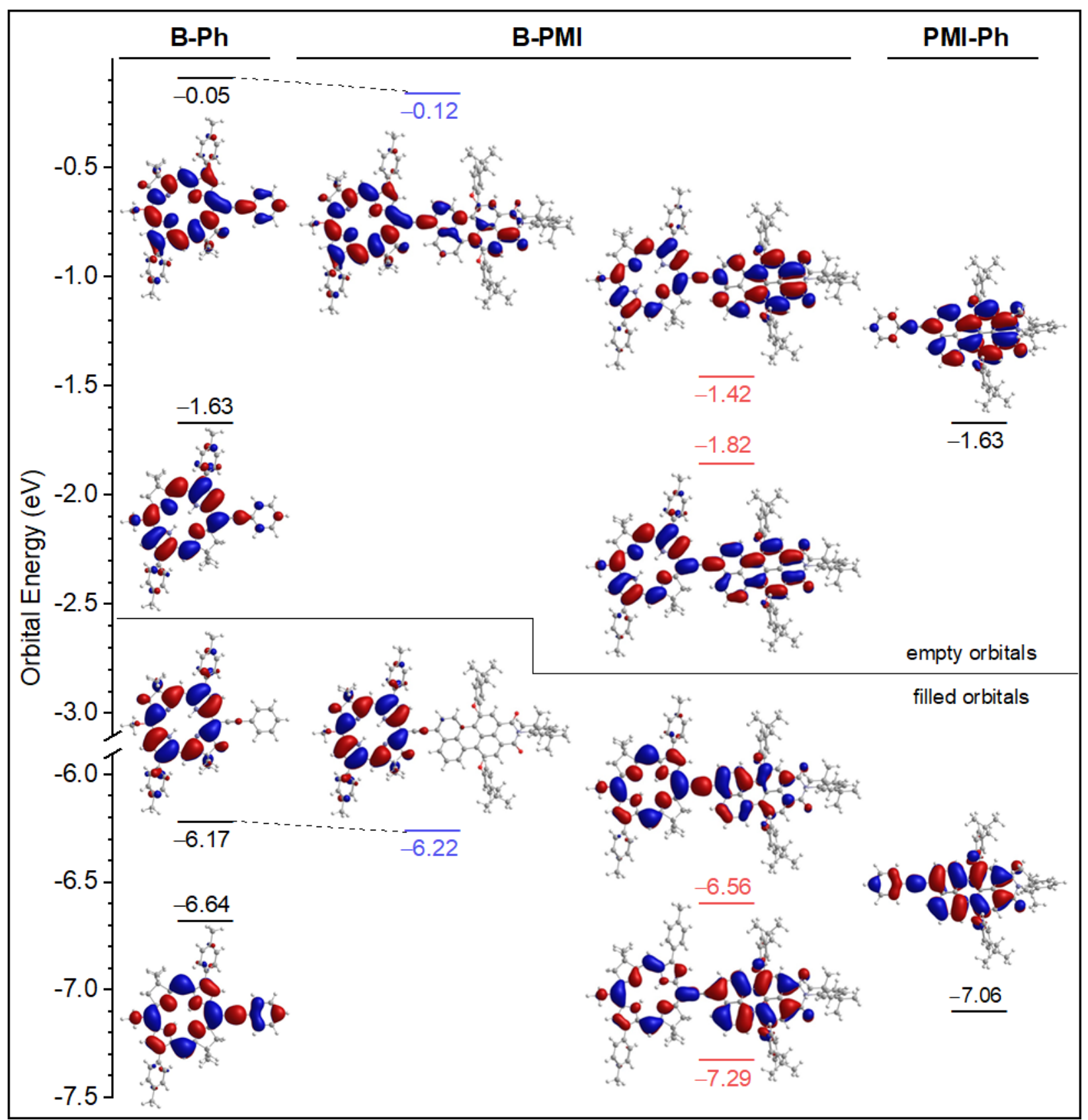

Figure S64. MO correlation diagram for dyad B-PMI, bacteriochlorin benchmark B-Ph and perylene benchmark PMI-Ph. The MOs for the dyad with energies indicated with blue font are essentially bacteriochlorin MOs. The MOs of the dyad with energies indicated with red font are mixtures of the corresponding orbitals of the perylene and bacteriochlorin. 


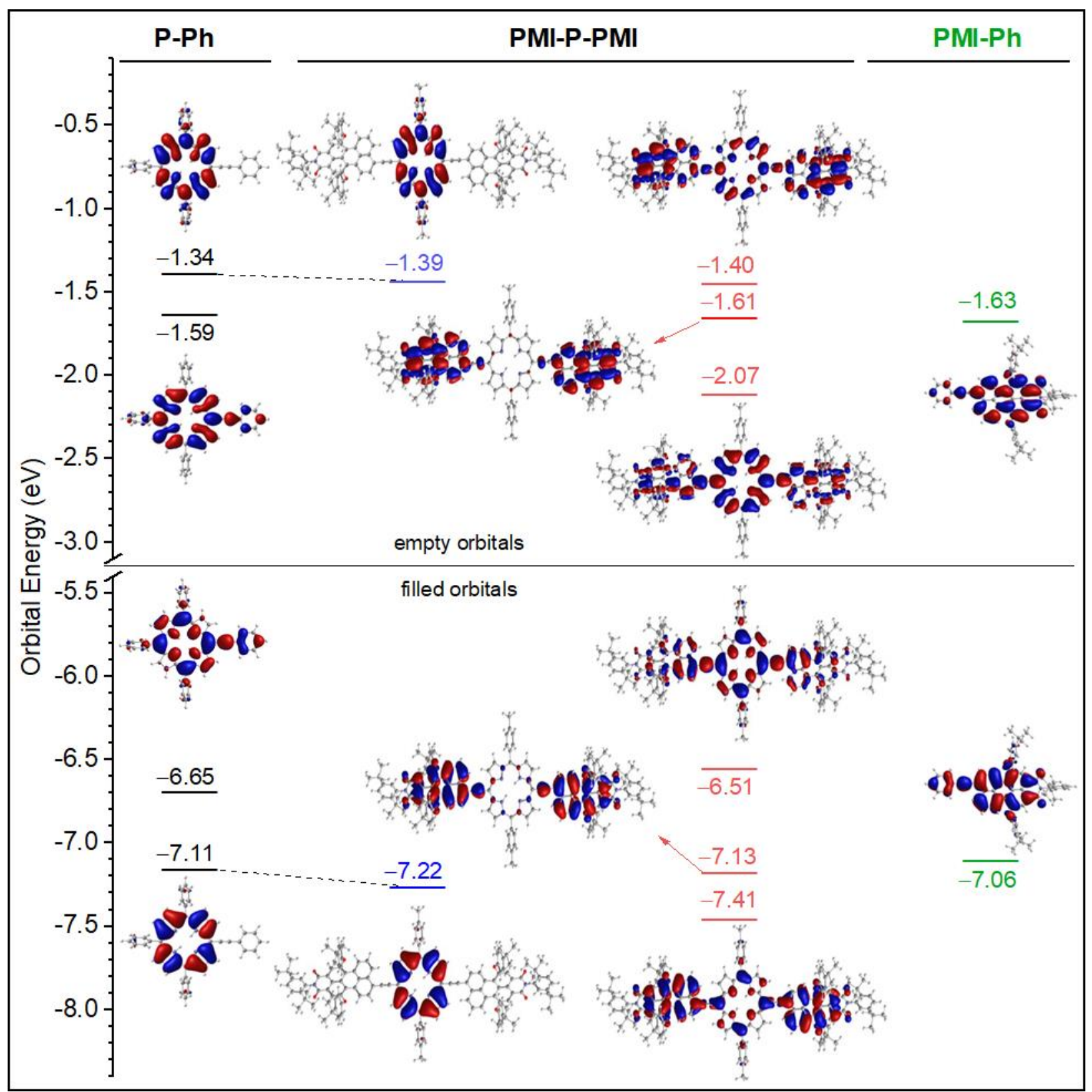

Figure S65. MO correlation diagram for triad PMI-P-PMI, porphyrin benchmark P-Ph, terrylene benchmark TMI-Ph and perylene benchmark PMI-Ph. The MOs for the triad with energies indicated with blue font are essentially porphyrin MOs (nodes at the linker attachment site); those with energies indicated in red font are mixtures (effectively bonding, nonbonding and antibonding trios) of the terrylene, perylene and porphyrin orbitals. 
Section S6

Excited-State

Compositions

From

TDDFT 
Table S3. TDDFT results for P-TMI in toluene. ${ }^{a}$

\begin{tabular}{|c|c|c|c|c|c|c|}
\hline State & $\underset{(\mathrm{nm})}{\lambda}$ & $\begin{array}{l}\text { Shifted } \lambda^{b} \\
(\mathrm{~nm})\end{array}$ & $\mathrm{f}$ & $\begin{array}{l}\text { filled } \\
\text { MO }\end{array}$ & $\begin{array}{l}\text { empty } \\
\text { MO }\end{array}$ & $\%$ \\
\hline \multirow[t]{7}{*}{$\mathrm{S}_{1}$} & 676 & 718 & 1.7 & $\mathrm{H}-2$ & $\mathrm{~L}+1$ & 2 \\
\hline & & & & H-2 & $\mathrm{L}+2$ & 9 \\
\hline & & & & H-1 & $\mathrm{L}+1$ & 19 \\
\hline & & & & H-1 & $\mathrm{L}+2$ & 2 \\
\hline & & & & $\mathrm{H}$ & $\mathrm{L}$ & 55 \\
\hline & & & & $\mathrm{H}$ & $\mathrm{L}+1$ & 6 \\
\hline & & & & $\mathrm{H}$ & $\mathrm{L}+2$ & 3 \\
\hline \multirow[t]{7}{*}{$\mathrm{S}_{2}$} & 619 & 653 & 0.66 & $\mathrm{H}-2$ & $\mathrm{~L}$ & 9 \\
\hline & & & & $\mathrm{H}-2$ & $\mathrm{~L}+1$ & 20 \\
\hline & & & & H-1 & $\mathrm{L}$ & 9 \\
\hline & & & & $\mathrm{H}-1$ & $\mathrm{~L}+2$ & 17 \\
\hline & & & & $\mathrm{H}$ & $\mathrm{L}$ & 15 \\
\hline & & & & $\mathrm{H}$ & $\mathrm{L}+1$ & 3 \\
\hline & & & & $\mathrm{H}$ & $\mathrm{L}+2$ & 23 \\
\hline \multirow[t]{8}{*}{$\mathrm{S}_{3}$} & 576 & 605 & 0.26 & H-2 & $\mathrm{L}$ & 4 \\
\hline & & & & $\mathrm{H}-2$ & $\mathrm{~L}+1$ & 6 \\
\hline & & & & H-2 & $\mathrm{L}+2$ & 14 \\
\hline & & & & H-1 & $\mathrm{L}$ & 26 \\
\hline & & & & $\mathrm{H}-1$ & $\mathrm{~L}+2$ & 5 \\
\hline & & & & $\mathrm{H}$ & $\mathrm{L}$ & 3 \\
\hline & & & & $\mathrm{H}$ & $\mathrm{L}+1$ & 30 \\
\hline & & & & $\mathrm{H}$ & $\mathrm{L}+2$ & 6 \\
\hline \multirow[t]{8}{*}{$\mathrm{S}_{4}$} & 424 & 439 & 0.94 & $\mathrm{H}-4$ & $\mathrm{~L}$ & 5 \\
\hline & & & & H-3 & $\mathrm{L}$ & 3 \\
\hline & & & & $\mathrm{H}-2$ & $\mathrm{~L}+2$ & 46 \\
\hline & & & & H-1 & $\mathrm{L}$ & 9 \\
\hline & & & & H-1 & $\mathrm{L}+1$ & 3 \\
\hline & & & & $\mathrm{H}$ & $\mathrm{L}$ & 5 \\
\hline & & & & $\mathrm{H}$ & $\mathrm{L}+1$ & 12 \\
\hline & & & & $\mathrm{H}-1$ & $\mathrm{~L}+3$ & 8 \\
\hline \multirow[t]{4}{*}{$\mathrm{S}_{5}$} & 410 & 424 & 1.6 & $\mathrm{H}-2$ & $\mathrm{~L}$ & 22 \\
\hline & & & & $\mathrm{H}-2$ & $\mathrm{~L}+1$ & 33 \\
\hline & & & & $\mathrm{H}-1$ & $\mathrm{~L}+2$ & 12 \\
\hline & & & & $\mathrm{H}$ & $\mathrm{L}+2$ & 29 \\
\hline
\end{tabular}

${ }^{a} \mathrm{H}=\mathrm{HOMO}$ and $\mathrm{L}=\mathrm{LUMO} .{ }^{b}$ Shifted to improve overlap with the measured spectrum for plotting. 
Table S4. TDDFT results for C-TMI in toluene. ${ }^{a}$

\begin{tabular}{|c|c|c|c|c|c|c|}
\hline State & $\underset{(\mathrm{nm})}{\lambda}$ & $\begin{array}{l}\text { Shifted } \lambda^{b} \\
(\mathrm{~nm})\end{array}$ & $\mathrm{f}$ & $\begin{array}{l}\text { filled } \\
\text { MO }\end{array}$ & $\begin{array}{c}\text { empty } \\
\text { MO }\end{array}$ & $\%$ \\
\hline \multirow[t]{4}{*}{$\mathrm{S}_{1}$} & 644 & 710 & 2.6 & $\mathrm{H}-2$ & $\mathrm{~L}$ & 6 \\
\hline & & & & $\mathrm{H}-2$ & $\mathrm{~L}+1$ & 7 \\
\hline & & & & $\mathrm{H}-1$ & $\mathrm{~L}+1$ & 7 \\
\hline & & & & $\mathrm{H}$ & $\mathrm{L}$ & 74 \\
\hline \multirow[t]{6}{*}{$\mathrm{S}_{2}$} & 616 & 676 & 0.13 & $\mathrm{H}-2$ & $\mathrm{~L}+2$ & 12 \\
\hline & & & & H-1 & $\mathrm{L}$ & 17 \\
\hline & & & & $\mathrm{H}-1$ & $\mathrm{~L}+1$ & 48 \\
\hline & & & & $\mathrm{H}$ & $\mathrm{L}$ & 4 \\
\hline & & & & $\mathrm{H}$ & $\mathrm{L}+1$ & 5 \\
\hline & & & & $\mathrm{H}$ & $\mathrm{L}+2$ & 10 \\
\hline \multirow[t]{5}{*}{$\mathrm{S}_{3}$} & 527 & 571 & 0.05 & $\mathrm{H}-2$ & $\mathrm{~L}$ & 21 \\
\hline & & & & $\mathrm{H}-2$ & $\mathrm{~L}+1$ & 17 \\
\hline & & & & H-1 & $\mathrm{L}+1$ & 2 \\
\hline & & & & H-1 & $\mathrm{L}+2$ & 15 \\
\hline & & & & $\mathrm{H}$ & $\mathrm{L}+1$ & 39 \\
\hline \multirow[t]{9}{*}{$\mathrm{S}_{4}$} & 412 & 438 & 0.56 & $\mathrm{H}-4$ & $\mathrm{~L}$ & 9 \\
\hline & & & & H-3 & $\mathrm{L}$ & 3 \\
\hline & & & & $\mathrm{H}-2$ & $\mathrm{~L}$ & 18 \\
\hline & & & & $\mathrm{H}-2$ & $\mathrm{~L}+1$ & 6 \\
\hline & & & & $\mathrm{H}-2$ & $\mathrm{~L}+2$ & 3 \\
\hline & & & & $\mathrm{H}-1$ & $\mathrm{~L}+2$ & 28 \\
\hline & & & & $\mathrm{H}$ & $\mathrm{L}$ & 3 \\
\hline & & & & $\mathrm{H}$ & $\mathrm{L}+2$ & 2 \\
\hline & & & & $\mathrm{H}$ & $\mathrm{L}+3$ & 18 \\
\hline \multirow[t]{7}{*}{$\mathrm{S}_{5}$} & 387 & 410 & 1.1 & $\mathrm{H}-2$ & $\mathrm{~L}+1$ & 5 \\
\hline & & & & $\mathrm{H}-2$ & $\mathrm{~L}+2$ & 9 \\
\hline & & & & H-1 & $\mathrm{L}$ & 11 \\
\hline & & & & $\mathrm{H}-1$ & $\mathrm{~L}+1$ & 7 \\
\hline & & & & $\mathrm{H}-1$ & $\mathrm{~L}+2$ & 26 \\
\hline & & & & $\mathrm{H}$ & $\mathrm{L}+1$ & 9 \\
\hline & & & & $\mathrm{H}$ & $\mathrm{L}+2$ & 29 \\
\hline
\end{tabular}

${ }^{a} \mathrm{H}=\mathrm{HOMO}$ and $\mathrm{L}=\mathrm{LUMO} .{ }^{b}$ Shifted to improve overlap with the measured spectrum for plotting. 
Table S5. TDDFT results for B-TMI in toluene. ${ }^{a}$

\begin{tabular}{|c|c|c|c|c|c|c|}
\hline State & $\underset{(\mathrm{nm})}{\lambda}$ & $\begin{array}{l}\text { Shifted } \lambda^{b} \\
(\mathrm{~nm})\end{array}$ & $\mathrm{f}$ & $\begin{array}{c}\text { filled } \\
\text { MO }\end{array}$ & $\begin{array}{c}\text { empty } \\
\text { MO }\end{array}$ & $\%$ \\
\hline \multirow[t]{5}{*}{$\mathrm{S}_{1}$} & 764 & 814 & 0.76 & $\mathrm{H}-2$ & $\mathrm{~L}+3$ & 3 \\
\hline & & & & $\mathrm{H}-1$ & $\mathrm{~L}+1$ & 3 \\
\hline & & & & $\mathrm{H}-1$ & $\mathrm{~L}+3$ & 3 \\
\hline & & & & $\mathrm{H}$ & $\mathrm{L}$ & 38 \\
\hline & & & & $\mathrm{H}$ & $\mathrm{L}+1$ & 50 \\
\hline \multirow[t]{3}{*}{$\mathrm{S}_{2}$} & 651 & 687 & 2.2 & $\mathrm{H}-2$ & $\mathrm{~L}+1$ & 15 \\
\hline & & & & $\mathrm{H}-1$ & $\mathrm{~L}$ & 70 \\
\hline & & & & $\mathrm{H}$ & $\mathrm{L}$ & 5 \\
\hline \multirow[t]{5}{*}{$\mathrm{S}_{3}$} & 560 & 587 & 0.18 & $\mathrm{H}-2$ & $\mathrm{~L}$ & 32 \\
\hline & & & & $\mathrm{H}-2$ & $\mathrm{~L}+1$ & 6 \\
\hline & & & & H-1 & $\mathrm{L}+1$ & 44 \\
\hline & & & & $\mathrm{H}$ & $\mathrm{L}+1$ & 2 \\
\hline & & & & $\mathrm{H}$ & $\mathrm{L}+3$ & 9 \\
\hline \multirow[t]{6}{*}{$\mathrm{S}_{4}$} & 408 & 422 & 0.25 & H-3 & $\mathrm{L}$ & 16 \\
\hline & & & & $\mathrm{H}-2$ & $\mathrm{~L}$ & 9 \\
\hline & & & & $\mathrm{H}-2$ & $\mathrm{~L}+1$ & 18 \\
\hline & & & & H-1 & $\mathrm{L}$ & 3 \\
\hline & & & & $\mathrm{H}$ & $\mathrm{L}+1$ & 16 \\
\hline & & & & $\mathrm{H}$ & $\mathrm{L}+3$ & 7 \\
\hline \multirow[t]{7}{*}{$\mathrm{S}_{5}$} & 395 & 408 & 0.17 & $\mathrm{H}-2$ & $\mathrm{~L}$ & 3 \\
\hline & & & & $\mathrm{H}-1$ & $\mathrm{~L}$ & 7 \\
\hline & & & & $\mathrm{H}-1$ & $\mathrm{~L}+1$ & 5 \\
\hline & & & & $\mathrm{H}$ & $\mathrm{L}$ & 35 \\
\hline & & & & $\mathrm{H}$ & $\mathrm{L}+1$ & 27 \\
\hline & & & & $\mathrm{H}$ & $\mathrm{L}+2$ & 11 \\
\hline & & & & $\mathrm{H}$ & $\mathrm{L}+3$ & 5 \\
\hline \multirow[t]{10}{*}{$\mathrm{S}_{10}$} & 364 & 350 & 1.5 & H-6 & $\mathrm{L}$ & 4 \\
\hline & & & & H-6 & $\mathrm{L}+1$ & 5 \\
\hline & & & & H-3 & $\mathrm{L}$ & 2 \\
\hline & & & & $\mathrm{H}-2$ & $\mathrm{~L}+2$ & 6 \\
\hline & & & & $\mathrm{H}-2$ & $\mathrm{~L}+3$ & 23 \\
\hline & & & & H-1 & $\mathrm{L}+2$ & 3 \\
\hline & & & & $\mathrm{H}-1$ & $\mathrm{~L}+3$ & 19 \\
\hline & & & & $\mathrm{H}-1$ & L+4 & 8 \\
\hline & & & & $\mathrm{H}$ & $\mathrm{L}+1$ & 6 \\
\hline & & & & $\mathrm{H}$ & $\mathrm{L}+3$ & 5 \\
\hline
\end{tabular}

${ }^{a} \mathrm{H}=\mathrm{HOMO}$ and $\mathrm{L}=\mathrm{LUMO} .{ }^{b}$ Shifted to improve overlap with the measured spectrum for plotting. 
Table S6. TDDFT results for PMI-P-TMI in toluene. ${ }^{a}$

\begin{tabular}{|c|c|c|c|c|c|c|}
\hline State & $\underset{(\mathrm{nm})}{\lambda}$ & $\begin{array}{l}\text { Shifted } \lambda^{b} \\
(\mathrm{~nm})\end{array}$ & $\mathrm{f}$ & $\begin{array}{c}\text { filled } \\
\text { MO }\end{array}$ & $\begin{array}{l}\text { empty } \\
\text { MO }\end{array}$ & $\%$ \\
\hline \multirow[t]{8}{*}{$\mathrm{S}_{1}$} & 728 & 755 & 2.4 & $\mathrm{H}-3$ & $\mathrm{~L}+2$ & 3 \\
\hline & & & & $\mathrm{H}-2$ & $\mathrm{~L}$ & 2 \\
\hline & & & & $\mathrm{H}-2$ & $\mathrm{~L}+3$ & 8 \\
\hline & & & & $\mathrm{H}-1$ & $\mathrm{~L}$ & 4 \\
\hline & & & & H-1 & $\mathrm{L}+1$ & 11 \\
\hline & & & & $\mathrm{H}$ & $\mathrm{L}$ & 60 \\
\hline & & & & $\mathrm{H}$ & $\mathrm{L}+2$ & 3 \\
\hline & & & & $\mathrm{H}$ & $\mathrm{L}+3$ & 2 \\
\hline \multirow[t]{10}{*}{$\mathrm{S}_{2}$} & 629 & 650 & 0.61 & H-3 & $\mathrm{L}+3$ & 5 \\
\hline & & & & $\mathrm{H}-2$ & $\mathrm{~L}$ & 28 \\
\hline & & & & $\mathrm{H}-2$ & $\mathrm{~L}+1$ & 4 \\
\hline & & & & $\mathrm{H}-2$ & $\mathrm{~L}+2$ & 7 \\
\hline & & & & $\mathrm{H}-1$ & $\mathrm{~L}$ & 2 \\
\hline & & & & H-1 & $\mathrm{L}+1$ & 4 \\
\hline & & & & $\mathrm{H}-1$ & $\mathrm{~L}+3$ & 10 \\
\hline & & & & $\mathrm{H}$ & $\mathrm{L}$ & 7 \\
\hline & & & & $\mathrm{H}$ & $\mathrm{L}+1$ & 4 \\
\hline & & & & $\mathrm{H}$ & $\mathrm{L}+3$ & 27 \\
\hline \multirow[t]{7}{*}{$\mathrm{S}_{3}$} & 595 & 613 & 0.37 & $\mathrm{H}-2$ & $\mathrm{~L}$ & 4 \\
\hline & & & & $\mathrm{H}-2$ & $\mathrm{~L}+3$ & 6 \\
\hline & & & & H-1 & $\mathrm{L}$ & 29 \\
\hline & & & & H-1 & $\mathrm{L}+1$ & 7 \\
\hline & & & & $\mathrm{H}$ & $\mathrm{L}+1$ & 34 \\
\hline & & & & $\mathrm{H}$ & $\mathrm{L}+2$ & 3 \\
\hline & & & & $\mathrm{H}$ & $\mathrm{L}+3$ & 4 \\
\hline \multirow[t]{8}{*}{$\mathrm{S}_{4}$} & 502 & 515 & 1.5 & H-3 & $\mathrm{L}$ & 17 \\
\hline & & & & H-3 & $\mathrm{L}+1$ & 13 \\
\hline & & & & H-3 & $\mathrm{L}+2$ & 15 \\
\hline & & & & $\mathrm{H}-2$ & $\mathrm{~L}+3$ & 10 \\
\hline & & & & $\mathrm{H}-1$ & $\mathrm{~L}$ & 3 \\
\hline & & & & $\mathrm{H}-1$ & $\mathrm{~L}+1$ & 8 \\
\hline & & & & $\mathrm{H}-1$ & $\mathrm{~L}+2$ & 12 \\
\hline & & & & $\mathrm{H}$ & $\mathrm{L}+2$ & 13 \\
\hline See & Next & Page & & & & \\
\hline
\end{tabular}




\begin{tabular}{|c|c|c|c|c|c|c|}
\hline \multirow[t]{12}{*}{$\mathrm{S}_{5}$} & \multirow[t]{12}{*}{425} & \multirow[t]{12}{*}{434} & \multirow[t]{12}{*}{0.67} & H-6 & $\mathrm{L}$ & 3 \\
\hline & & & & H-4 & $\mathrm{L}$ & 2 \\
\hline & & & & H-3 & $\mathrm{L}$ & 2 \\
\hline & & & & $\mathrm{H}-2$ & L & 5 \\
\hline & & & & $\mathrm{H}-2$ & $\mathrm{~L}+3$ & 35 \\
\hline & & & & H-1 & $\mathrm{L}+1$ & 9 \\
\hline & & & & H-1 & $\mathrm{L}+4$ & 2 \\
\hline & & & & $\mathrm{H}$ & $\mathrm{L}$ & 8 \\
\hline & & & & $\mathrm{H}$ & $\mathrm{L}+1$ & 3 \\
\hline & & & & $\mathrm{H}$ & $\mathrm{L}+2$ & 4 \\
\hline & & & & $\mathrm{H}$ & $\mathrm{L}+3$ & 7 \\
\hline & & & & $\mathrm{H}$ & $\mathrm{L}+4$ & 6 \\
\hline \multirow[t]{9}{*}{$\mathrm{S}_{6}$} & \multirow[t]{9}{*}{421} & \multirow[t]{9}{*}{430} & \multirow[t]{9}{*}{1.4} & H-3 & $\mathrm{L}+3$ & 4 \\
\hline & & & & $\mathrm{H}-2$ & $\mathrm{~L}$ & 33 \\
\hline & & & & $\mathrm{H}-2$ & $\mathrm{~L}+1$ & 4 \\
\hline & & & & $\mathrm{H}-2$ & $\mathrm{~L}+2$ & 6 \\
\hline & & & & $\mathrm{H}-2$ & $\mathrm{~L}+3$ & 4 \\
\hline & & & & $\mathrm{H}-1$ & $\mathrm{~L}+1$ & 2 \\
\hline & & & & H-1 & $\mathrm{L}+1$ & 7 \\
\hline & & & & $\mathrm{H}$ & $\mathrm{L}$ & 2 \\
\hline & & & & $\mathrm{H}$ & $\mathrm{L}+3$ & 28 \\
\hline \multirow[t]{8}{*}{$S_{7}$} & \multirow[t]{8}{*}{397} & \multirow[t]{8}{*}{405} & \multirow[t]{8}{*}{0.35} & H-6 & $\mathrm{L}$ & 3 \\
\hline & & & & H-4 & $\mathrm{L}$ & 2 \\
\hline & & & & H-3 & $\mathrm{L}+2$ & 7 \\
\hline & & & & $\mathrm{H}-2$ & $L+3$ & 24 \\
\hline & & & & H-1 & $\mathrm{L}$ & 25 \\
\hline & & & & H-1 & $\mathrm{L}+2$ & 15 \\
\hline & & & & $\mathrm{H}$ & $\mathrm{L}+1$ & 3 \\
\hline & & & & $\mathrm{H}$ & $\mathrm{L}+4$ & 4 \\
\hline
\end{tabular}

${ }^{a} \mathrm{H}=\mathrm{HOMO}$ and $\mathrm{L}=\mathrm{LUMO} .{ }^{b}$ Shifted to improve overlap with the measured spectrum for plotting. 
Table S7. TDDFT results for P-PMI in toluene. ${ }^{a}$

\begin{tabular}{|c|c|c|c|c|c|c|}
\hline State & $\underset{(\mathrm{nm})}{\lambda}$ & $\begin{array}{c}\text { Shifted } \lambda^{b} \\
(\mathrm{~nm})\end{array}$ & $\mathrm{F}$ & $\begin{array}{l}\text { filled } \\
\text { MO }\end{array}$ & $\begin{array}{l}\text { empty } \\
\text { MO }\end{array}$ & $\%$ \\
\hline \multirow[t]{8}{*}{$\mathrm{S}_{1}$} & 653 & 692 & 0.52 & $\mathrm{H}-2$ & $\mathrm{~L}+1$ & 4 \\
\hline & & & & H-2 & $\mathrm{L}+2$ & 3 \\
\hline & & & & H-1 & $\mathrm{L}$ & 8 \\
\hline & & & & $\mathrm{H}-1$ & $\mathrm{~L}+1$ & 5 \\
\hline & & & & $\mathrm{H}-1$ & $\mathrm{~L}+2$ & 13 \\
\hline & & & & $\mathrm{H}$ & $\mathrm{L}$ & 41 \\
\hline & & & & $\mathrm{H}$ & $\mathrm{L}+1$ & 11 \\
\hline & & & & $\mathrm{H}$ & $\mathrm{L}+2$ & 14 \\
\hline \multirow[t]{8}{*}{$\mathrm{S}_{2}$} & 599 & 631 & 0.40 & H-2 & $\mathrm{L}+1$ & 3 \\
\hline & & & & $\mathrm{H}-2$ & $\mathrm{~L}+2$ & 4 \\
\hline & & & & $\mathrm{H}-1$ & $\mathrm{~L}$ & 19 \\
\hline & & & & H-1 & $\mathrm{L}+1$ & 11 \\
\hline & & & & H-1 & $\mathrm{L}+2$ & 4 \\
\hline & & & & $\mathrm{H}$ & $\mathrm{L}$ & 20 \\
\hline & & & & $\mathrm{H}$ & $\mathrm{L}+1$ & 2 \\
\hline & & & & $\mathrm{H}$ & $\mathrm{L}+2$ & 34 \\
\hline \multirow[t]{5}{*}{$\mathrm{S}_{3}$} & 501 & 523 & 1.5 & H-2 & $\mathrm{L}$ & 34 \\
\hline & & & & H-2 & $\mathrm{L}+1$ & 10 \\
\hline & & & & H-1 & $\mathrm{L}+2$ & 16 \\
\hline & & & & $\mathrm{H}$ & $\mathrm{L}$ & 12 \\
\hline & & & & $\mathrm{H}$ & $\mathrm{L}+1$ & 23 \\
\hline \multirow[t]{5}{*}{$\mathrm{S}_{4}$} & 410 & 425 & 1.5 & $\mathrm{H}-2$ & $\mathrm{~L}$ & 2 \\
\hline & & & & H-1 & $\mathrm{L}$ & 32 \\
\hline & & & & $\mathrm{H}-1$ & $\mathrm{~L}+1$ & 15 \\
\hline & & & & $\mathrm{H}-1$ & $\mathrm{~L}+2$ & 9 \\
\hline & & & & $\mathrm{H}$ & $\mathrm{L}+2$ & 34 \\
\hline \multirow[t]{8}{*}{$\mathrm{S}_{5}$} & 403 & 418 & 1.2 & $\mathrm{H}-2$ & $\mathrm{~L}$ & 7 \\
\hline & & & & H-2 & $\mathrm{L}+1$ & 5 \\
\hline & & & & $\mathrm{H}-1$ & $\mathrm{~L}$ & 7 \\
\hline & & & & H-1 & $\mathrm{L}+1$ & 2 \\
\hline & & & & H-1 & $\mathrm{L}+2$ & 50 \\
\hline & & & & $\mathrm{H}$ & $\mathrm{L}$ & 8 \\
\hline & & & & $\mathrm{H}$ & $\mathrm{L}+1$ & 10 \\
\hline & & & & $\mathrm{H}$ & $\mathrm{L}+2$ & 6 \\
\hline
\end{tabular}

${ }^{a} \mathrm{H}=\mathrm{HOMO}$ and $\mathrm{L}=\mathrm{LUMO} .{ }^{b}$ Shifted to improve overlap with the measured spectrum for plotting. 
Table S8. TDDFT results for C-PMI in toluene. ${ }^{a}$

\begin{tabular}{|c|c|c|c|c|c|c|}
\hline State & $\begin{array}{c}\lambda \\
(\mathrm{nm})\end{array}$ & $\begin{array}{l}\text { Shifted } \lambda^{b} \\
\quad(\mathrm{~nm})\end{array}$ & $\mathrm{f}$ & $\begin{array}{l}\text { filled } \\
\text { MO }\end{array}$ & $\begin{array}{l}\text { empty } \\
\text { MO }\end{array}$ & $\%$ \\
\hline \multirow[t]{7}{*}{$\mathrm{S}_{1}$} & 619 & 677 & 0.24 & $\mathrm{H}-2$ & $\mathrm{~L}+2$ & 5 \\
\hline & & & & H-1 & $\mathrm{L}$ & 30 \\
\hline & & & & H-1 & $\mathrm{L}+1$ & 27 \\
\hline & & & & $\mathrm{H}-1$ & +2 & 3 \\
\hline & & & & $\mathrm{H}$ & $\mathrm{L}$ & 11 \\
\hline & & & & $\mathrm{H}$ & $\mathrm{L}+1$ & 9 \\
\hline & & & & $\mathrm{H}$ & $\mathrm{L}+2$ & 13 \\
\hline \multirow[t]{6}{*}{$\mathrm{S}_{2}$} & 563 & 612 & 1.6 & $\mathrm{H}-2$ & $\mathrm{~L}+1$ & 12 \\
\hline & & & & $\mathrm{H}-1$ & $\mathrm{~L}$ & 9 \\
\hline & & & & $\mathrm{H}-1$ & $\mathrm{~L}+1$ & 4 \\
\hline & & & & $\mathrm{H}-1$ & $\mathrm{~L}+2$ & 3 \\
\hline & & & & $\mathrm{H}$ & $\mathrm{L}$ & 63 \\
\hline & & & & $\mathrm{H}$ & $\mathrm{L}+2$ & 3 \\
\hline \multirow[t]{5}{*}{$\mathrm{S}_{3}$} & 488 & 524 & 0.55 & $\mathrm{H}-2$ & $\mathrm{~L}$ & 29 \\
\hline & & & & H-1 & $\mathrm{L}+1$ & 7 \\
\hline & & & & H-1 & $\mathrm{L}+2$ & 14 \\
\hline & & & & $\mathrm{H}$ & $\mathrm{L}$ & 3 \\
\hline & & & & $\mathrm{H}$ & $\mathrm{L}+1$ & 40 \\
\hline \multirow[t]{7}{*}{$\mathrm{S}_{4}$} & 389 & 412 & 1.3 & $\mathrm{H}-2$ & $\mathrm{~L}$ & 10 \\
\hline & & & & $\mathrm{H}-1$ & $\mathrm{~L}$ & 3 \\
\hline & & & & H-1 & $\mathrm{L}+2$ & 60 \\
\hline & & & & $\mathrm{H}$ & $\mathrm{L}$ & 4 \\
\hline & & & & $\mathrm{H}$ & $\mathrm{L}+1$ & 9 \\
\hline & & & & $\mathrm{H}$ & $\mathrm{L}+2$ & 7 \\
\hline & & & & $\mathrm{H}$ & $\mathrm{L}+3$ & 3 \\
\hline \multirow[t]{8}{*}{$\mathrm{S}_{5}$} & 383 & 404 & 1.0 & $\mathrm{H}-2$ & $\mathrm{~L}$ & 5 \\
\hline & & & & $\mathrm{H}-2$ & $\mathrm{~L}+1$ & 4 \\
\hline & & & & H-2 & $\mathrm{L}+2$ & 8 \\
\hline & & & & $\mathrm{H}-1$ & $\mathrm{~L}$ & 15 \\
\hline & & & & H-1 & $\mathrm{L}+1$ & 8 \\
\hline & & & & H-1 & $\mathrm{L}+2$ & 3 \\
\hline & & & & $\mathrm{H}$ & $\mathrm{L}$ & 2 \\
\hline & & & & $\mathrm{H}$ & $\mathrm{L}+2$ & 49 \\
\hline
\end{tabular}

${ }^{a} \mathrm{H}=\mathrm{HOMO}$ and $\mathrm{L}=\mathrm{LUMO} .{ }^{b}$ Shifted to improve overlap with the measured spectrum for plotting. 
Table S9. TDDFT results for B-PMI in toluene. ${ }^{a}$

\begin{tabular}{|ccccccc|}
\hline State & $\begin{array}{c}\lambda \\
(\mathrm{nm})\end{array}$ & $\begin{array}{c}\text { Shifted } \lambda^{b} \\
(\mathrm{~nm})\end{array}$ & $\mathrm{f}$ & $\begin{array}{c}\text { filled } \\
\text { MO }\end{array}$ & $\begin{array}{c}\text { empty } \\
\text { MO }\end{array}$ & $\%$ \\
\hline $\mathrm{S}_{1}$ & 763 & 794 & 0.64 & $\mathrm{H}-1$ & $\mathrm{~L}+2$ & 4 \\
& & & & $\mathrm{H}$ & $\mathrm{L}$ & 70 \\
& & & & $\mathrm{H}$ & $\mathrm{L}+1$ & 23 \\
\hline $\mathrm{S}_{2}$ & 599 & 618 & 1.1 & $\mathrm{H}-2$ & $\mathrm{~L}+1$ & 10 \\
& & & & $\mathrm{H}-1$ & $\mathrm{~L}$ & 74 \\
& & & & $\mathrm{H}-1$ & $\mathrm{~L}+1$ & 3 \\
& & & & $\mathrm{H}$ & $\mathrm{L}+2$ & 8 \\
\hline $\mathrm{S}_{3}$ & 496 & 509 & 0.94 & $\mathrm{H}-2$ & $\mathrm{~L}$ & 29 \\
& & & & $\mathrm{H}-2$ & $\mathrm{~L}+1$ & 10 \\
& & & & $\mathrm{H}-1$ & $\mathrm{~L}+1$ & 47 \\
& & & & $\mathrm{H}$ & $\mathrm{L}+2$ & 5 \\
\hline $\mathrm{S}_{4}$ & 385 & 392 & 0.44 & $\mathrm{H}-1$ & $\mathrm{~L}$ & 8 \\
& & & & $\mathrm{H}-1$ & $\mathrm{~L} 1$ & 3 \\
& & & & $\mathrm{H}$ & $\mathrm{L}$ & 14 \\
& & & & $\mathrm{H}$ & $\mathrm{L}+1$ & 43 \\
& & & & $\mathrm{H}$ & $\mathrm{L}+2$ & 27 \\
\hline $\mathrm{S}_{5}$ & 372 & 379 & 0.41 & $\mathrm{H}-8$ & $\mathrm{~L}$ & 6 \\
& & & & $\mathrm{H}-8$ & $\mathrm{~L}+1$ & 3 \\
& & & & $\mathrm{H}-4$ & $\mathrm{~L}$ & 2 \\
& & & & $\mathrm{H}-2$ & $\mathrm{~L}$ & 6 \\
& & & & $\mathrm{H}-2$ & $\mathrm{~L}+1$ & 24 \\
& & & & $\mathrm{H}-1$ & $\mathrm{~L}$ & 3 \\
& & & & $\mathrm{H}-1$ & $\mathrm{~L}+2$ & 23 \\
& & & & $\mathrm{H}$ & $\mathrm{L}$ & 7 \\
& & & & $\mathrm{H}$ & $\mathrm{L}+1$ & 4 \\
& & & & $\mathrm{H}$ & $\mathrm{L}+2$ & 5 \\
& & & $\mathrm{H}$ & $\mathrm{L}+3$ & 5 \\
\hline
\end{tabular}

${ }^{a} \mathrm{H}=\mathrm{HOMO}$ and $\mathrm{L}=\mathrm{LUMO} .{ }^{b}$ Shifted to improve overlap with the measured spectrum for plotting. 
Table S10. TDDFT Results for PMI-P-PMI in Toluene ${ }^{a}$

\begin{tabular}{|c|c|c|c|c|c|c|}
\hline State & $\stackrel{\lambda}{\lambda}$ & $\begin{array}{c}\text { Shifted } \\
\lambda^{b} \\
(\mathrm{~nm})\end{array}$ & $\mathrm{f}$ & $\begin{array}{c}\text { filled } \\
\text { MO }\end{array}$ & $\begin{array}{c}\text { empty } \\
\text { MO }\end{array}$ & $\%$ \\
\hline \multirow[t]{7}{*}{$\mathrm{S}_{1}$} & 710 & 752 & 1.5 & H-3 & $\mathrm{L}+2$ & 3 \\
\hline & & & & H-2 & $\mathrm{L}$ & 4 \\
\hline & & & & H-2 & $\mathrm{L}+3$ & 10 \\
\hline & & & & H-1 & $\mathrm{L}+1$ & 3 \\
\hline & & & & $\mathrm{H}$ & $\mathrm{L}$ & 65 \\
\hline & & & & $\mathrm{H}$ & $\mathrm{L}+2$ & 4 \\
\hline & & & & $\mathrm{H}$ & $\mathrm{L}+3$ & 5 \\
\hline \multirow[t]{5}{*}{$\mathrm{S}_{2}$} & 622 & 654 & 0.34 & H-3 & $\mathrm{L}+3$ & 6 \\
\hline & & & & $\mathrm{H}-2$ & $\mathrm{~L}$ & 35 \\
\hline & & & & $\mathrm{H}-2$ & $\mathrm{~L}+2$ & 7 \\
\hline & & & & $\mathrm{H}$ & $\mathrm{L}$ & 8 \\
\hline & & & & $\mathrm{H}$ & $\mathrm{L}+3$ & 38 \\
\hline \multirow[t]{4}{*}{$\mathrm{S}_{3}$} & 505 & 526 & 0.02 & H-3 & $\mathrm{L}+1$ & 17 \\
\hline & & & & H-1 & $\mathrm{L}$ & 32 \\
\hline & & & & H-1 & $\mathrm{L}+2$ & 15 \\
\hline & & & & $\mathrm{H}$ & $\mathrm{L}+1$ & 30 \\
\hline \multirow[t]{6}{*}{$\mathrm{S}_{4}$} & 497 & 518 & 2.6 & H-3 & $\mathrm{L}$ & 17 \\
\hline & & & & H-3 & $\mathrm{L}+2$ & 5 \\
\hline & & & & H-2 & $\mathrm{L}+3$ & 20 \\
\hline & & & & H-1 & $\mathrm{L}+1$ & 31 \\
\hline & & & & $\mathrm{H}$ & $\mathrm{L}$ & 3 \\
\hline & & & & $\mathrm{H}$ & $\mathrm{L}+2$ & 19 \\
\hline \multirow[t]{4}{*}{$\mathrm{S}_{5}$} & 420 & 435 & 1.4 & $\mathrm{H}-3$ & $\mathrm{~L}+3$ & 3 \\
\hline & & & & $\mathrm{H}-2$ & $\mathrm{~L}$ & 42 \\
\hline & & & & $\mathrm{H}-2$ & $\mathrm{~L}+2$ & 8 \\
\hline & & & & $\mathrm{H}$ & $\mathrm{L}+3$ & 43 \\
\hline \multirow[t]{6}{*}{$\mathrm{S}_{6}$} & 406 & 420 & 0.77 & H-3 & $\mathrm{L}$ & 2 \\
\hline & & & & H-3 & $\mathrm{L}+2$ & 5 \\
\hline & & & & $\mathrm{H}-2$ & $\mathrm{~L}+3$ & 59 \\
\hline & & & & H-1 & $\mathrm{L}+1$ & 9 \\
\hline & & & & $\mathrm{H}$ & $\mathrm{L}$ & 9 \\
\hline & & & & $\mathrm{H}$ & $\mathrm{L}+2$ & 8 \\
\hline
\end{tabular}

${ }^{a} \mathrm{H}=\mathrm{HOMO}$ and $\mathrm{L}=$ LUMO. ${ }^{b}$ Shifted by $800 \mathrm{~cm}^{-1}$ to improve overlap with the measured spectrum for plotting. 
Table S11. TDDFT results for $\mathbf{P}-\mathbf{P h}$ in toluene. ${ }^{a}$

\begin{tabular}{|ccccccc|}
\hline State & $\begin{array}{c}\lambda \\
(\mathrm{nm})\end{array}$ & $\begin{array}{c}\text { Shifted } \lambda^{b} \\
(\mathrm{~nm})\end{array}$ & $\mathrm{f}$ & $\begin{array}{c}\text { filled } \\
\text { MO }\end{array}$ & $\begin{array}{c}\text { empty } \\
\text { MO }\end{array}$ & $\%$ \\
\hline $\mathrm{S}_{1}$ & 641 & 671 & 0.11 & $\mathrm{H}-1$ & $\mathrm{~L}$ & 21 \\
& & & & $\mathrm{H}-1$ & $\mathrm{~L}+1$ & 13 \\
& & & & $\mathrm{H}$ & $\mathrm{L}$ & 36 \\
& & & & $\mathrm{H}$ & $\mathrm{L}+1$ & 29 \\
\hline $\mathrm{S}_{2}$ & 587 & 612 & 0.16 & $\mathrm{H}-1$ & $\mathrm{~L}$ & 22 \\
& & & & $\mathrm{H}-1$ & $\mathrm{~L}+1$ & 13 \\
& & & & $\mathrm{H}$ & $\mathrm{L}$ & 37 \\
& & & & $\mathrm{H}$ & $\mathrm{L}+1$ & 28 \\
\hline $\mathrm{S}_{3}$ & 408 & 420 & 2.0 & $\mathrm{H}-1$ & $\mathrm{~L}$ & 19 \\
& & & & $\mathrm{H}-1$ & $\mathrm{~L}+1$ & 48 \\
& & & & $\mathrm{H}$ & $\mathrm{L}$ & 16 \\
& & & & $\mathrm{H}$ & $\mathrm{L}+1$ & 14 \\
\hline $\mathrm{S}_{4}$ & 403 & 415 & 1.9 & $\mathrm{H}-1$ & $\mathrm{~L}$ & 38 \\
& & & & $\mathrm{H}-1$ & $\mathrm{~L}+1$ & 24 \\
& & & & $\mathrm{H}$ & $\mathrm{L}$ & 10 \\
& & & & $\mathrm{H}$ & $\mathrm{L}+1$ & 28 \\
\hline $\mathrm{S}_{6}$ & 319 & 326 & 0.27 & $\mathrm{H}-4$ & $\mathrm{~L}$ & 31 \\
& & & & $\mathrm{H}-4$ & $\mathrm{~L}+1$ & 22 \\
& & & & $\mathrm{H}-3$ & $\mathrm{~L}+2$ & 4 \\
& & & & H-2 & L & 24 \\
& & & & H & L+2 & 6 \\
\hline
\end{tabular}

${ }^{a} \mathrm{H}=\mathrm{HOMO}$ and $\mathrm{L}=\mathrm{LUMO} .{ }^{b}$ Shifted to improve overlap with the measured spectrum for plotting. 
Table S12. TDDFT results for $\mathbf{C}$-Ph in toluene. ${ }^{a}$

\begin{tabular}{|ccccccc|}
\hline State & $\begin{array}{c}\lambda \\
(\mathrm{nm})\end{array}$ & $\begin{array}{c}\text { Shifted } \lambda \\
(\mathrm{nm})\end{array}$ & $\mathrm{f}$ & $\begin{array}{c}\text { filled } \\
\text { MO }\end{array}$ & $\begin{array}{c}\text { empty } \\
\text { MO }\end{array}$ & $\%$ \\
\hline $\mathrm{S}_{1}$ & 616 & 665 & 0.14 & $\mathrm{H}-1$ & $\mathrm{~L}$ & 37 \\
& & & & $\mathrm{H}-1$ & +1 & 10 \\
& & & & $\mathrm{H}$ & $\mathrm{L}$ & 37 \\
& & & & $\mathrm{H}$ & $\mathrm{L}+1$ & 14 \\
\hline $\mathrm{S}_{2}$ & 529 & 565 & 0.37 & $\mathrm{H}-1$ & $\mathrm{~L}$ & 35 \\
& & & & $\mathrm{H}-1$ & $\mathrm{~L}+1$ & 9 \\
& & & & $\mathrm{H}$ & $\mathrm{L}$ & 42 \\
& & & & $\mathrm{H}$ & $\mathrm{L}+1$ & 11 \\
\hline $\mathrm{S}_{3}$ & 389 & 408 & 2.0 & $\mathrm{H}-1$ & $\mathrm{~L}$ & 8 \\
& & & & $\mathrm{H}-1$ & $\mathrm{~L}+1$ & 49 \\
& & & & $\mathrm{H}$ & $\mathrm{L}$ & 11 \\
& & & & $\mathrm{H}$ & $\mathrm{L}+1$ & 28 \\
\hline $\mathrm{S}_{4}$ & 379 & 397 & 1.5 & $\mathrm{H}-1$ & $\mathrm{~L}$ & 18 \\
& & & & $\mathrm{H}-1$ & $\mathrm{~L}+1$ & 28 \\
& & & & $\mathrm{H}$ & $\mathrm{L}$ & 8 \\
& & & & $\mathrm{H}$ & $\mathrm{L}+1$ & 43 \\
\hline $\mathrm{S}_{5}$ & 313 & 326 & 0.04 & $\mathrm{H}-6$ & $\mathrm{~L}$ & 3 \\
& & & & $\mathrm{H}-5$ & $\mathrm{~L}$ & 33 \\
& & & & $\mathrm{H}-2$ & $\mathrm{~L}$ & 49 \\
& & & & $\mathrm{H}-1$ & $\mathrm{~L}+2$ & 2 \\
& & & & $\mathrm{H}$ & $\mathrm{L}+2$ & 3 \\
\hline
\end{tabular}

${ }^{a} \mathrm{H}=\mathrm{HOMO}$ and $\mathrm{L}=\mathrm{LUMO} .{ }^{b}$ Shifted to improve overlap with the measured spectrum for plotting. 
Table S13. TDDFT results for B-Ph in toluene. ${ }^{a}$

\begin{tabular}{|ccccccc|}
\hline State & $\begin{array}{c}\lambda \\
(\mathrm{nm})\end{array}$ & $\begin{array}{c}\text { Shifted } \lambda^{b} \\
(\mathrm{~nm})\end{array}$ & $\mathrm{f}$ & $\begin{array}{c}\text { filled } \\
\text { MO }\end{array}$ & $\begin{array}{c}\text { empty } \\
\text { MO }\end{array}$ & $\%$ \\
\hline $\mathrm{S}_{1}$ & 756 & 756 & 0.58 & $\mathrm{H}-1$ & $\mathrm{~L}+1$ & 5 \\
& & & & $\mathrm{H}$ & $\mathrm{L}$ & 92 \\
\hline $\mathrm{S}_{2}$ & 572 & 572 & 0.39 & $\mathrm{H}-1$ & $\mathrm{~L}$ & 84 \\
& & & & $\mathrm{H}$ & $\mathrm{L}+1$ & 14 \\
\hline $\mathrm{S}_{3}$ & 367 & 367 & 1.6 & $\mathrm{H}-2$ & $\mathrm{~L}$ & 5 \\
& & & & $\mathrm{H}-1$ & $\mathrm{~L}$ & 13 \\
& & & & $\mathrm{H}-1$ & $\mathrm{~L}+1$ & 8 \\
& & & & $\mathrm{H}$ & $\mathrm{L}+1$ & 68 \\
& & & & $\mathrm{H}$ & $\mathrm{L}+1$ & 3 \\
\hline $\mathrm{S}_{4}$ & 345 & 345 & 1.8 & $\mathrm{H}-3$ & $\mathrm{~L}$ & 3 \\
& & & & $\mathrm{H}-1$ & $\mathrm{~L}$ & 2 \\
& & & & $\mathrm{H}-1$ & $\mathrm{~L}+1$ & 73 \\
& & & & $\mathrm{H}$ & $\mathrm{L}$ & 5 \\
$\mathrm{~S}_{6}$ & 320 & 327 & 0.09 & $\mathrm{H}-8$ & $\mathrm{~L}+1$ & 10 \\
& & & & $\mathrm{H}-4$ & $\mathrm{~L}$ & 6 \\
& & & & $\mathrm{H}-3$ & $\mathrm{~L}$ & 29 \\
& & & & $\mathrm{H}-2$ & $\mathrm{~L}$ & 38 \\
& & & & $\mathrm{H}-1$ & $\mathrm{~L}+1$ & 4 \\
& & & & $\mathrm{H}$ & $\mathrm{L}+1$ & 4 \\
\hline
\end{tabular}

${ }^{a} \mathrm{H}=\mathrm{HOMO}$ and $\mathrm{L}=\mathrm{LUMO} .{ }^{b}$ Shifted to improve overlap with the measured spectrum for plotting. 
Table S14. TDDFT results for PMI-Ph in toluene. ${ }^{a}$

\begin{tabular}{|c|c|c|c|c|c|c|}
\hline State & $\begin{array}{c}\lambda \\
(\mathrm{nm})\end{array}$ & $\begin{array}{c}\text { Shifted } \lambda^{b} \\
(\mathrm{~nm})\end{array}$ & $\mathrm{f}$ & $\begin{array}{c}\text { filled } \\
\text { MO }\end{array}$ & $\begin{array}{c}\text { empty } \\
\text { MO }\end{array}$ & $\%$ \\
\hline $\mathrm{S}_{1}$ & 493 & 535 & 1.3 & $\mathrm{H}$ & $\mathrm{L}$ & 95 \\
\hline \multirow[t]{6}{*}{$\mathrm{S}_{2}$} & 357 & 379 & 0.24 & H-9 & $\mathrm{L}$ & 5 \\
\hline & & & & $\mathrm{H}-7$ & $\mathrm{~L}$ & 9 \\
\hline & & & & H-1 & $\mathrm{L}$ & 64 \\
\hline & & & & $\mathrm{H}$ & $\mathrm{L}+1$ & 4 \\
\hline & & & & $\mathrm{H}$ & $\mathrm{L}+2$ & 3 \\
\hline & & & & $\mathrm{H}$ & $\mathrm{L}+3$ & 3 \\
\hline \multirow[t]{5}{*}{$\mathrm{S}_{3}$} & 335 & 353 & 0.19 & H-12 & $\mathrm{L}$ & 5 \\
\hline & & & & $\mathrm{H}-4$ & $\mathrm{~L}$ & 3 \\
\hline & & & & $\mathrm{H}-2$ & $\mathrm{~L}$ & 29 \\
\hline & & & & $\mathrm{H}$ & $\mathrm{L}+1$ & 48 \\
\hline & & & & $\mathrm{H}$ & $\mathrm{L}+2$ & 3 \\
\hline \multirow[t]{9}{*}{$\mathrm{S}_{4}$} & 315 & 332 & 0.03 & H-12 & $\mathrm{L}$ & 3 \\
\hline & & & & H-6 & $\mathrm{L}$ & 38 \\
\hline & & & & $\mathrm{H}-2$ & $\mathrm{~L}$ & 7 \\
\hline & & & & H-1 & $\mathrm{L}$ & 4 \\
\hline & & & & $\mathrm{H}$ & $\mathrm{L}+1$ & 3 \\
\hline & & & & $\mathrm{H}$ & $\mathrm{L}+2$ & 9 \\
\hline & & & & $\mathrm{H}$ & $\mathrm{L}+3$ & 9 \\
\hline & & & & $\mathrm{H}$ & $\mathrm{L}+6$ & 3 \\
\hline & & & & $\mathrm{H}$ & $\mathrm{L} 7$ & 7 \\
\hline \multirow[t]{6}{*}{$\mathrm{S}_{5}$} & 305 & 321 & 0.06 & H-11 & $\mathrm{L}$ & 9 \\
\hline & & & & H-6 & $\mathrm{L}$ & 16 \\
\hline & & & & H-2 & $\mathrm{L}$ & 11 \\
\hline & & & & $\mathrm{H}-2$ & $\mathrm{~L}+1$ & 3 \\
\hline & & & & $\mathrm{H}-1$ & $\mathrm{~L}$ & 3 \\
\hline & & & & $\mathrm{H}$ & $\mathrm{L}+2$ & 47 \\
\hline
\end{tabular}

${ }^{a} \mathrm{H}=\mathrm{HOMO}$ and $\mathrm{L}=\mathrm{LUMO}$. ${ }^{b}$ Shifted to improve overlap with the measured spectrum for plotting. 
Table S15. TDDFT results for TMI-Ph in toluene. ${ }^{a}$

\begin{tabular}{|c|c|c|c|c|c|c|}
\hline State & $\begin{array}{c}\lambda \\
(\mathrm{nm})\end{array}$ & $\begin{array}{l}\text { Shifted } \lambda^{b} \\
\quad(\mathrm{~nm})\end{array}$ & $\mathrm{f}$ & $\begin{array}{c}\text { filled } \\
\text { MO }\end{array}$ & $\begin{array}{c}\text { empty } \\
\text { MO }\end{array}$ & $\%$ \\
\hline \multirow[t]{2}{*}{$\mathrm{S}_{1}$} & 607 & 655 & 1.8 & H-1 & $\mathrm{L}+1$ & 2 \\
\hline & & & & $\mathrm{H}$ & $\mathrm{L}$ & 94 \\
\hline \multirow[t]{2}{*}{$\mathrm{S}_{2}$} & 389 & 408 & 0.06 & H-1 & $\mathrm{L}$ & 28 \\
\hline & & & & $\mathrm{H}$ & $\mathrm{L}+1$ & 61 \\
\hline \multirow[t]{7}{*}{$\mathrm{S}_{3}$} & 363 & 379 & 0.19 & H-12 & $\mathrm{L}$ & 2 \\
\hline & & & & H-8 & $\mathrm{L}$ & 6 \\
\hline & & & & $\mathrm{H}-5$ & $\mathrm{~L}$ & 3 \\
\hline & & & & $\mathrm{H}-2$ & $\mathrm{~L}$ & 42 \\
\hline & & & & $\mathrm{H}-2$ & $\mathrm{~L}+1$ & 5 \\
\hline & & & & $\mathrm{H}$ & $\mathrm{L}+2$ & 24 \\
\hline & & & & $\mathrm{H}$ & $\mathrm{L}+3$ & 2 \\
\hline \multirow[t]{6}{*}{$\mathrm{S}_{4}$} & 349 & 365 & 0.10 & H-12 & $\mathrm{L}$ & 4 \\
\hline & & & & H-9 & $\mathrm{L}$ & 4 \\
\hline & & & & $\mathrm{H}-5$ & $\mathrm{~L}$ & 10 \\
\hline & & & & $\mathrm{H}-2$ & $\mathrm{~L}$ & 21 \\
\hline & & & & $\mathrm{H}-2$ & $\mathrm{~L}+1$ & 3 \\
\hline & & & & $\mathrm{H}$ & $\mathrm{L}+2$ & 39 \\
\hline \multirow[t]{10}{*}{$\mathrm{S}_{8}$} & 308 & 320 & 0.18 & H-10 & $\mathrm{L}$ & 12 \\
\hline & & & & H-10 & $\mathrm{L}+1$ & 4 \\
\hline & & & & H-9 & $\mathrm{L}$ & 20 \\
\hline & & & & $\mathrm{H}-5$ & $\mathrm{~L}$ & 8 \\
\hline & & & & $\mathrm{H}-4$ & $\mathrm{~L}$ & 5 \\
\hline & & & & $\mathrm{H}-1$ & $\mathrm{~L}+1$ & 7 \\
\hline & & & & $\mathrm{H}$ & $\mathrm{L}+3$ & 10 \\
\hline & & & & $\mathrm{H}$ & $\mathrm{L}+4$ & 5 \\
\hline & & & & $\mathrm{H}$ & $\mathrm{L}+5$ & 5 \\
\hline & & & & $\mathrm{H}$ & $\mathrm{L}+18$ & 3 \\
\hline
\end{tabular}

${ }^{a} \mathrm{H}=\mathrm{HOMO}$ and L = LUMO. ${ }^{b}$ Shifted to improve overlap with the measured spectrum for plotting. 
Table S16. TDDFT Results for PMI-P-PMI in Toluene ${ }^{a}$

\begin{tabular}{|c|c|c|c|c|c|c|}
\hline State & $\begin{array}{c}\lambda \\
(\mathrm{nm})\end{array}$ & $\begin{array}{c}\text { Shifted } \\
\lambda^{b} \\
(\mathrm{~nm})\end{array}$ & $\mathrm{f}$ & $\begin{array}{c}\text { filled } \\
\mathrm{MO}\end{array}$ & $\begin{array}{c}\text { empty } \\
\text { MO }\end{array}$ & $\%$ \\
\hline \multirow[t]{7}{*}{$\mathrm{S}_{1}$} & 710 & 752 & 1.50 & $\mathrm{H}-3$ & $\mathrm{~L}+2$ & 3 \\
\hline & & & & H-2 & $\mathrm{L}$ & 4 \\
\hline & & & & H-2 & $\mathrm{L}+3$ & 10 \\
\hline & & & & H-1 & $\mathrm{L}+1$ & 3 \\
\hline & & & & $\mathrm{H}$ & $\mathrm{L}$ & 65 \\
\hline & & & & $\mathrm{H}$ & $\mathrm{L}+2$ & 4 \\
\hline & & & & $\mathrm{H}$ & $\mathrm{L}+3$ & 5 \\
\hline \multirow[t]{5}{*}{$\mathrm{S}_{2}$} & 622 & 654 & 0.34 & H-3 & $\mathrm{L}+3$ & 6 \\
\hline & & & & H-2 & $\mathrm{L}$ & 3 \\
\hline & & & & H-2 & $\mathrm{L}+2$ & 7 \\
\hline & & & & $\mathrm{H}$ & $\mathrm{L}$ & 8 \\
\hline & & & & $\mathrm{H}$ & $\mathrm{L}+3$ & 38 \\
\hline \multirow[t]{4}{*}{$\mathrm{S}_{3}$} & 505 & 526 & 0.02 & $\mathrm{H}-3$ & $\mathrm{~L}+1$ & 17 \\
\hline & & & & H-1 & $\mathrm{L}$ & 32 \\
\hline & & & & H-1 & $\mathrm{L}+2$ & 15 \\
\hline & & & & $\mathrm{H}$ & $\mathrm{L}+1$ & 30 \\
\hline \multirow[t]{6}{*}{$\mathrm{S}_{4}$} & 497 & 518 & 2.60 & H-3 & $\mathrm{L}$ & 17 \\
\hline & & & & H-3 & $\mathrm{L}+2$ & 5 \\
\hline & & & & H-2 & $\mathrm{L}+3$ & 20 \\
\hline & & & & H-1 & $\mathrm{L}+1$ & 31 \\
\hline & & & & $\mathrm{H}$ & $\mathrm{L}$ & 3 \\
\hline & & & & $\mathrm{H}$ & $\mathrm{L}+2$ & 19 \\
\hline \multirow[t]{4}{*}{$\mathrm{S}_{5}$} & 420 & 435 & 1.43 & H-3 & $\mathrm{L}+3$ & 3 \\
\hline & & & & H-2 & $\mathrm{L}$ & 42 \\
\hline & & & & H-2 & $\mathrm{L}+2$ & 8 \\
\hline & & & & $\mathrm{H}$ & $\mathrm{L}+3$ & 43 \\
\hline \multirow[t]{6}{*}{$\mathrm{S}_{6}$} & 406 & 420 & 0.77 & H-3 & $\mathrm{L}$ & 2 \\
\hline & & & & H-3 & $\mathrm{L}+2$ & 5 \\
\hline & & & & H-2 & $\mathrm{L}+3$ & 59 \\
\hline & & & & H-1 & $\mathrm{L}+1$ & 9 \\
\hline & & & & $\mathrm{H}$ & $\mathrm{L}$ & 9 \\
\hline & & & & $\mathrm{H}$ & $\mathrm{L}+2$ & 8 \\
\hline
\end{tabular}

${ }^{a} \mathrm{H}=\mathrm{HOMO}$ and $\mathrm{L}=\mathrm{LUMO} .{ }^{b}$ Shifted to improve overlap with the measured spectrum for plotting. 


\section{Section S7}

\section{Experimental}

vs

Calculated

Absorption Spectra and

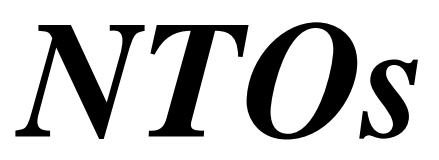




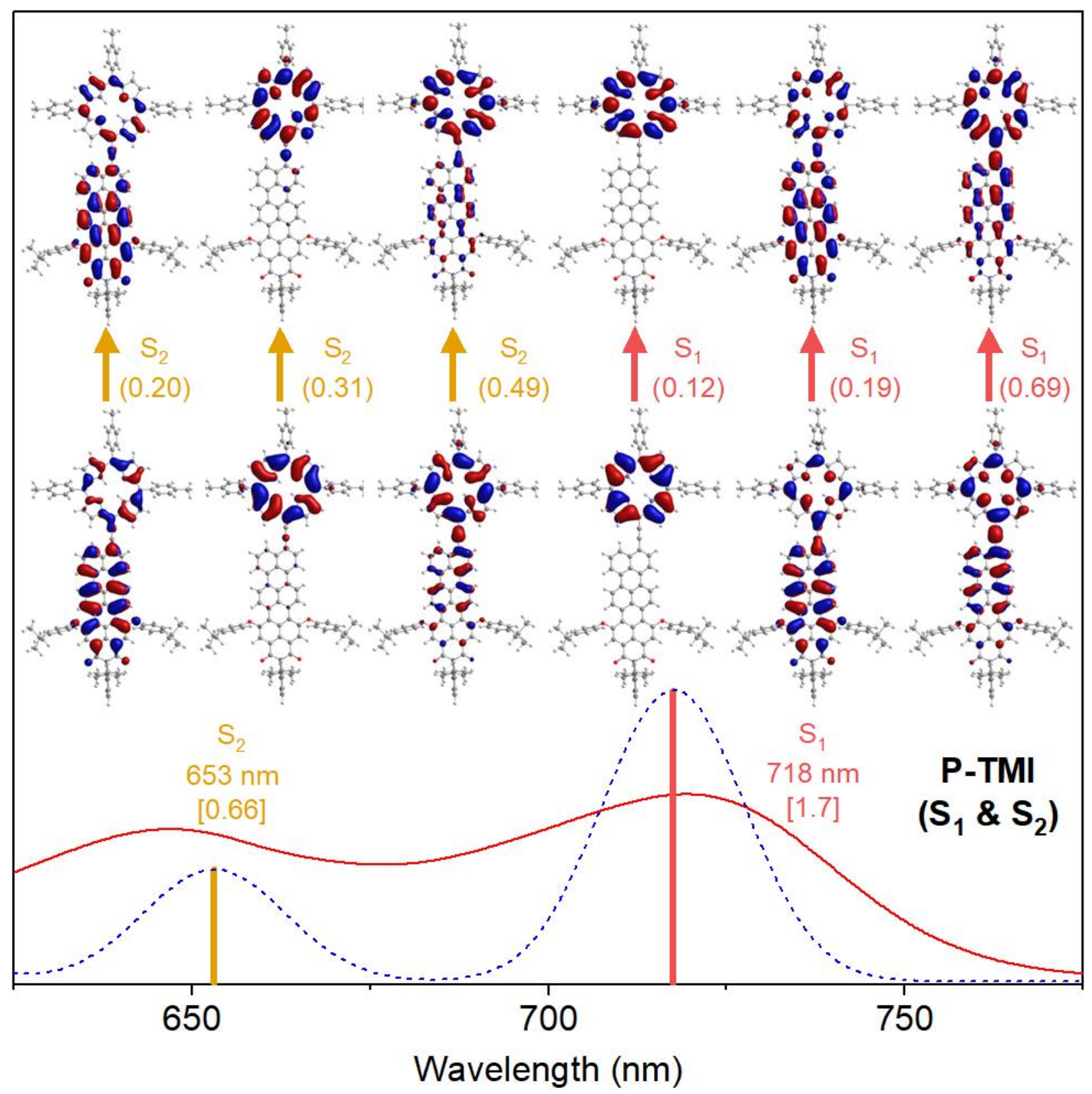

Figure S66. Comparison for dyad P-TMI of the absorption spectra in toluene that are measured (red line) or calculated via TDDFT (dashed blue lines and colored sticks) normalized at the highest peak. The calculated spectrum was shifted arbitrarily by $850 \mathrm{~cm}^{-1}$ to lower energy to better align with the measured spectrum. Features in the calculated spectra were given 10-nm Gaussian skirts. The wavelength and oscillator strength (in square brackets) of the transitions from the ground state to $S_{1}$ (red) and $S_{2}$ (gold) calculated from TDDFT are indicated. Also shown are the sets of occupied and virtual NTOs along with the eigenvalue (weight) for each transition. 


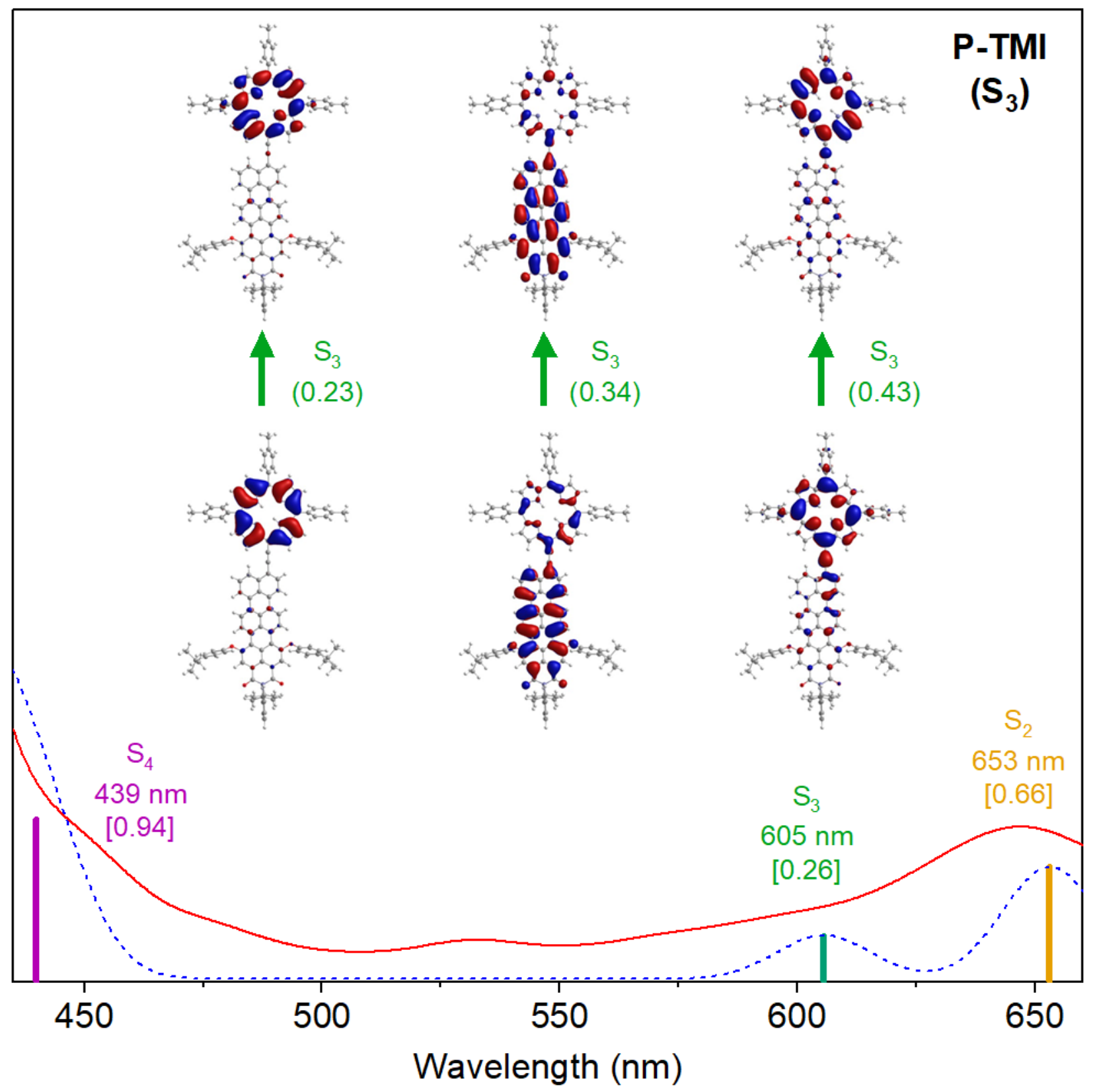

Figure S67. Comparison for dyad P-TMI of the absorption spectra in toluene that are measured (red line) or calculated via TDDFT (dashed blue lines and colored sticks) normalized at the highest peak. The calculated spectrum was shifted arbitrarily by $850 \mathrm{~cm}^{-1}$ to lower energy to better align with the measured spectrum. Features in the calculated spectra were given 10-nm Gaussian skirts. The wavelength and oscillator strength (in square brackets) of the transition from the ground state to $S_{3}$ (green) calculated from TDDFT is indicated. Also shown is the set of occupied and virtual NTOs along with the eigenvalue (weight) for the transition/ 


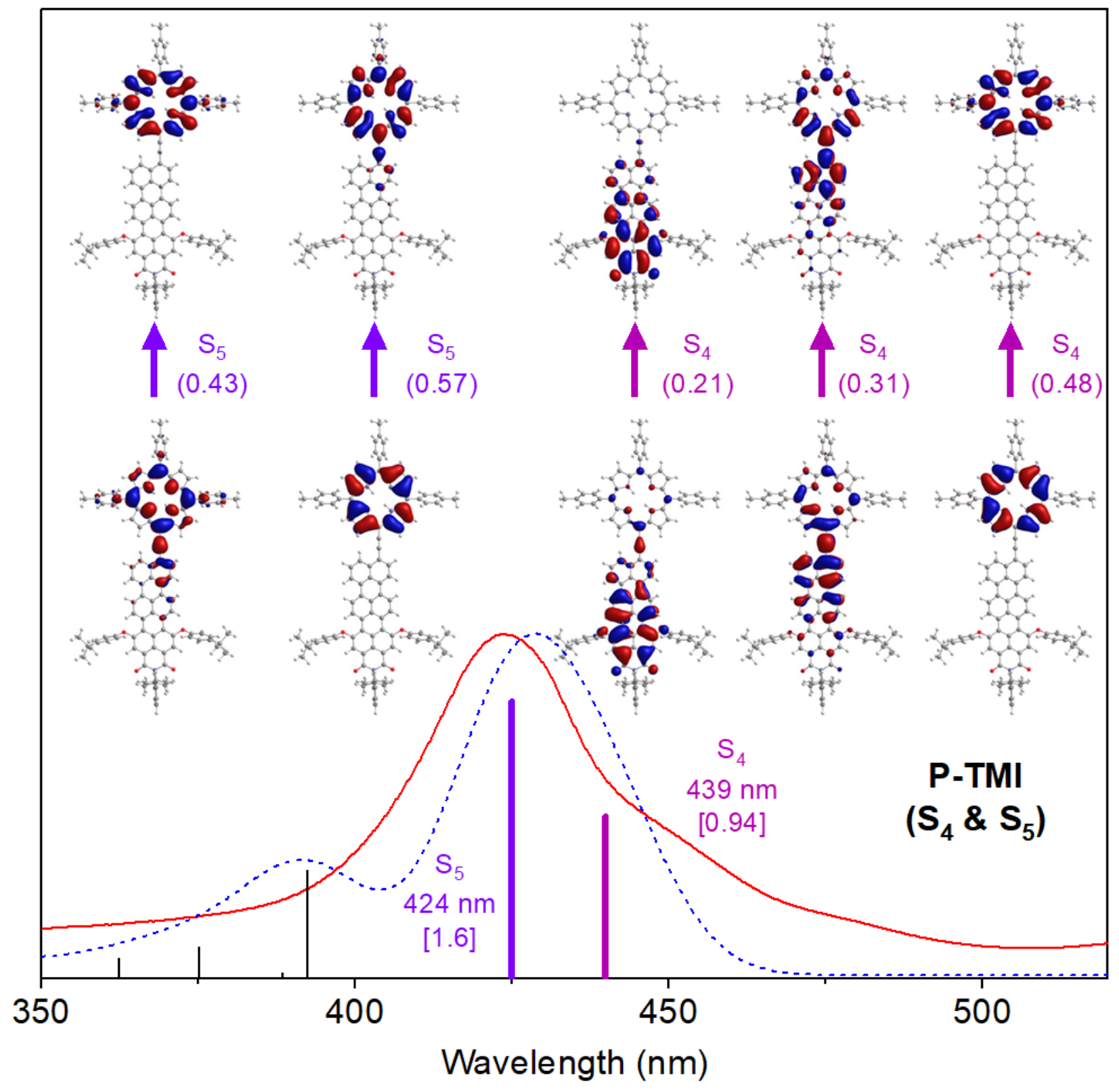

Figure S68. Comparison for dyad P-TMI of the absorption spectra in toluene that are measured (red line) or calculated via TDDFT (dashed blue lines and colored sticks) normalized at the highest peak. The calculated spectrum was shifted arbitrarily by $850 \mathrm{~cm}^{-1}$ to lower energy to better align with the measured spectrum. Features in the calculated spectra were given 10-nm Gaussian skirts. The wavelength and oscillator strength (in square brackets) of the transitions from the ground state to $S_{4}$ (mauve) and $S_{5}$ (violet) calculated from TDDFT are indicated. Also shown are the sets of occupied and virtual NTOs along with the eigenvalue (weight) for each transition. 


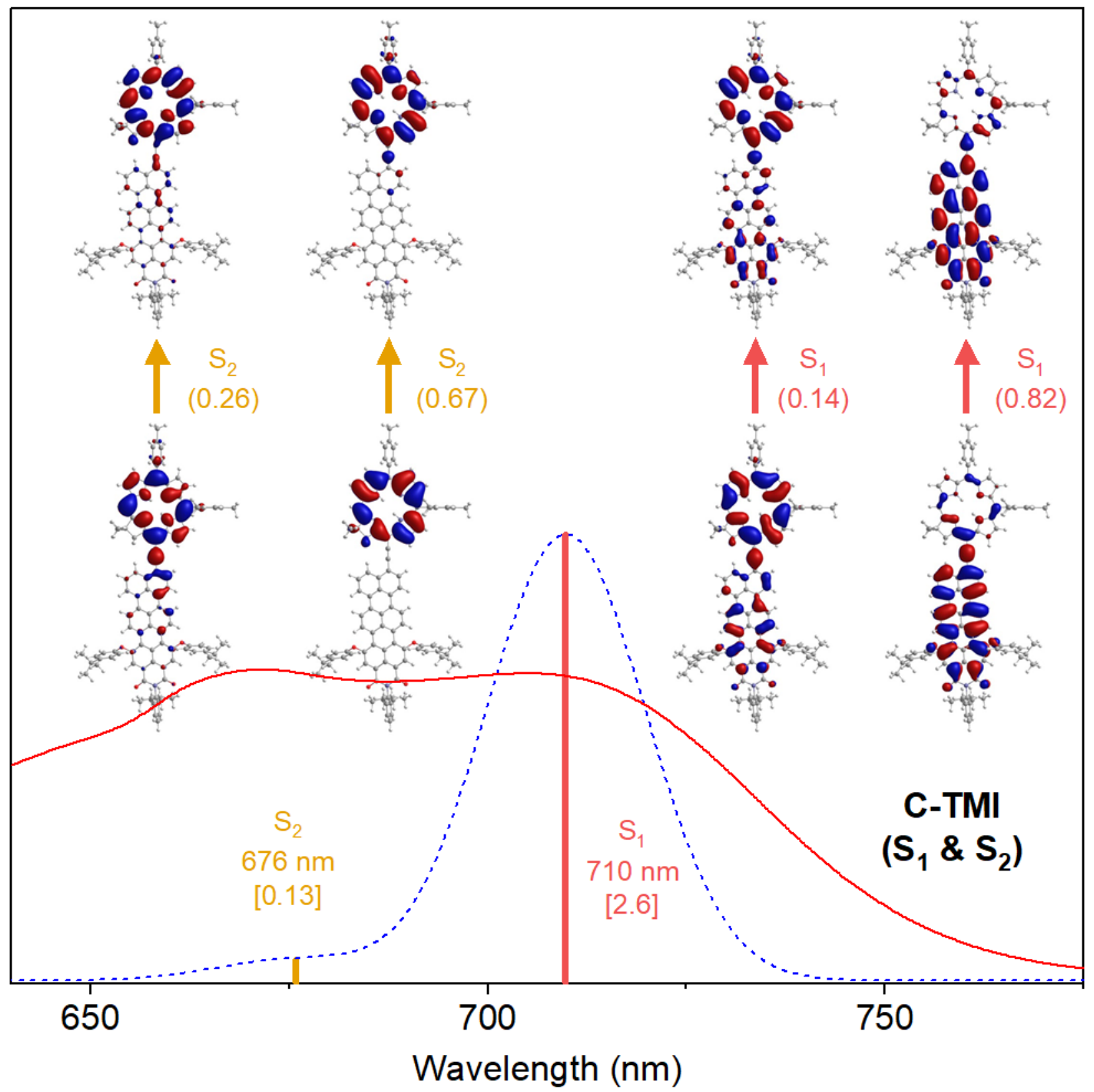

Figure S69. Comparison for dyad C-TMI of the absorption spectra in toluene that are measured (red line) or calculated via TDDFT (dashed blue lines and colored sticks) normalized at the highest peak. The calculated spectrum was shifted arbitrarily by $1450 \mathrm{~cm}^{-1}$ to lower energy to better align with the measured spectrum. Features in the calculated spectra were given 10-nm Gaussian skirts. The wavelength and oscillator strength (in square brackets) of the transitions from the ground state to $S_{1}$ (red) and $S_{2}$ (gold) calculated from TDDFT are indicated. Also shown are the sets of occupied and virtual NTOs along with the eigenvalue (weight) for each transition. 


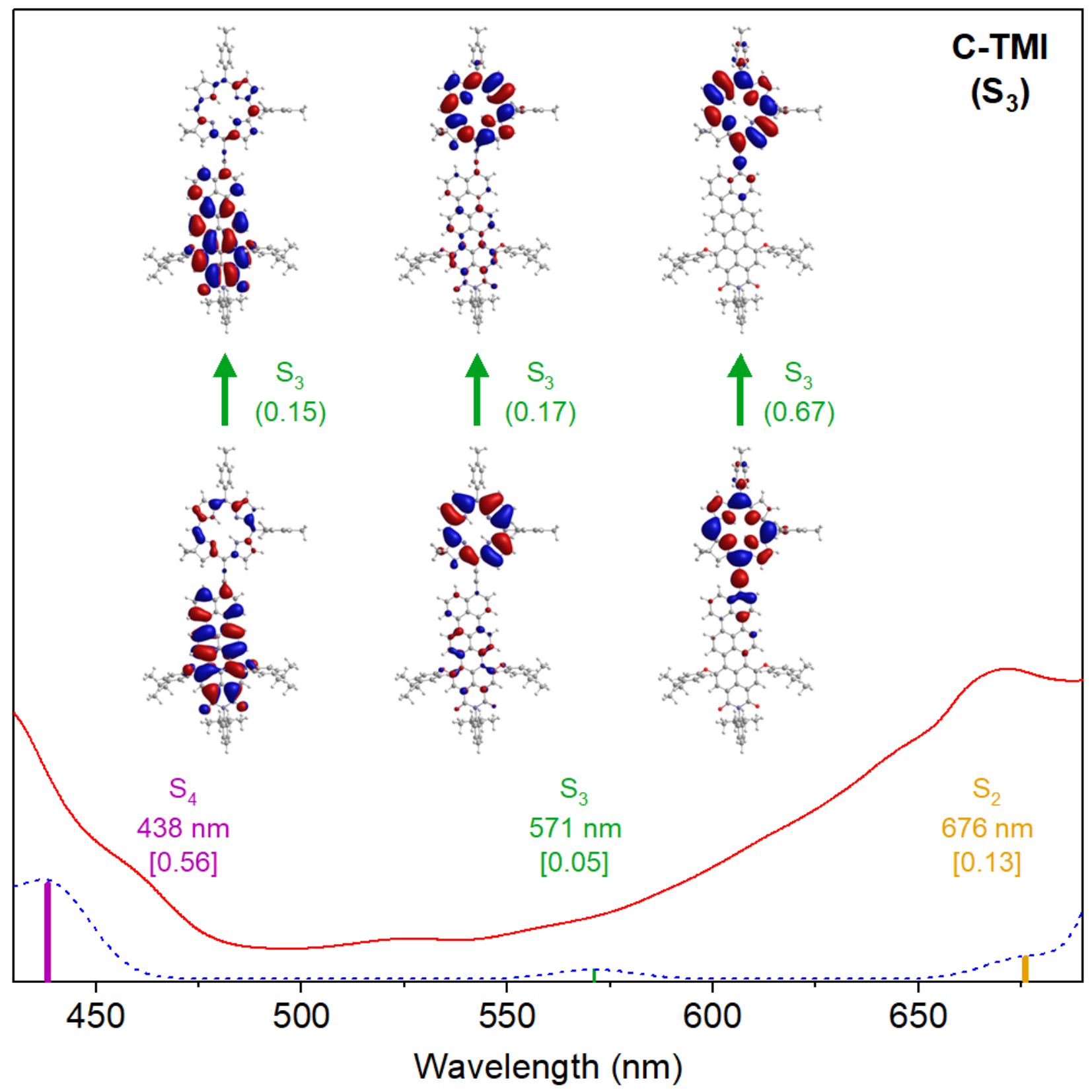

Figure S70. Comparison for dyad C-TMI of the absorption spectra in toluene that are measured (red line) or calculated via TDDFT (dashed blue lines and colored sticks) normalized at the highest peak. The calculated spectrum was shifted arbitrarily by $1450 \mathrm{~cm}^{-1}$ to lower energy to better align with the measured spectrum. Features in the calculated spectra were given 10-nm Gaussian skirts. The wavelength and oscillator strength (in square brackets) of the transition from the ground state to $S_{3}$ (green) calculated from TDDFT is indicated. Also shown is the set of occupied and virtual NTOs along with the eigenvalue (weight) for the transition. 


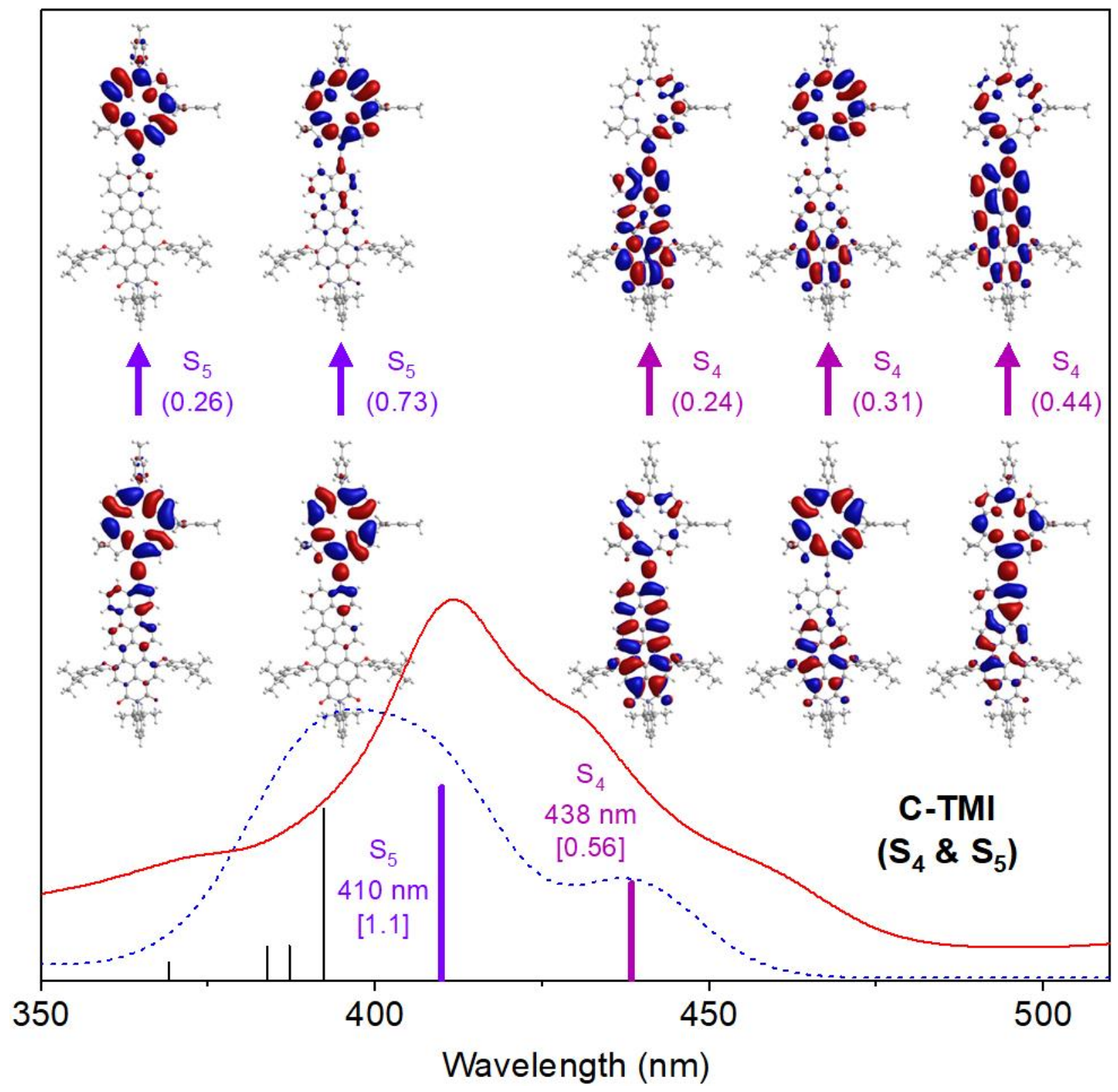

Figure S71. Comparison for dyad C-TMI of the absorption spectra in toluene that are measured (red line) or calculated via TDDFT (dashed blue lines and colored sticks) normalized at the highest peak. The calculated spectrum was shifted arbitrarily by $1450 \mathrm{~cm}^{-1}$ to lower energy to better align with the measured spectrum. Features in the calculated spectra were given 10-nm Gaussian skirts. The wavelength and oscillator strength (in square brackets) of the transitions from the ground state to $S_{4}$ (mauve) and $S_{5}$ (violet) calculated from TDDFT are indicated. Also shown are the sets of occupied and virtual NTOs along with the eigenvalue (weight) for each transition. 


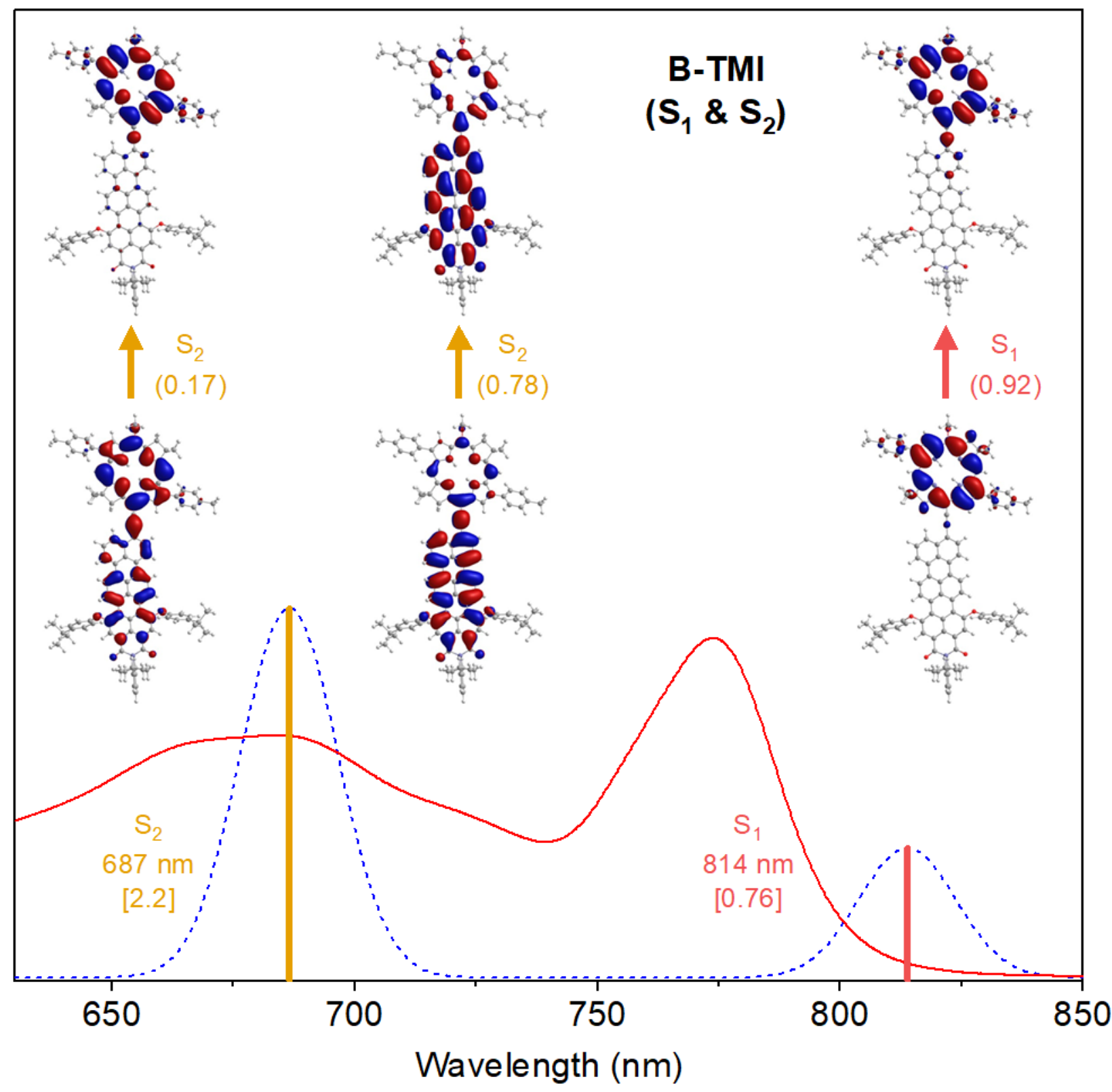

Figure S72. Comparison for dyad B-TMI of the absorption spectra in toluene that are measured (red line) or calculated via TDDFT (dashed blue lines and colored sticks) normalized at the highest peak. The calculated spectrum was shifted arbitrarily by $800 \mathrm{~cm}^{-1}$ to lower energy to better align with the measured spectrum. Features in the calculated spectra were given 10-nm Gaussian skirts. The wavelength and oscillator strength (in square brackets) of the transitions from the ground state to $S_{1}$ (red) and $S_{2}$ (gold) calculated from TDDFT are indicated. Also shown are the sets of occupied and virtual NTOs along with the eigenvalue (weight) for each transition. 


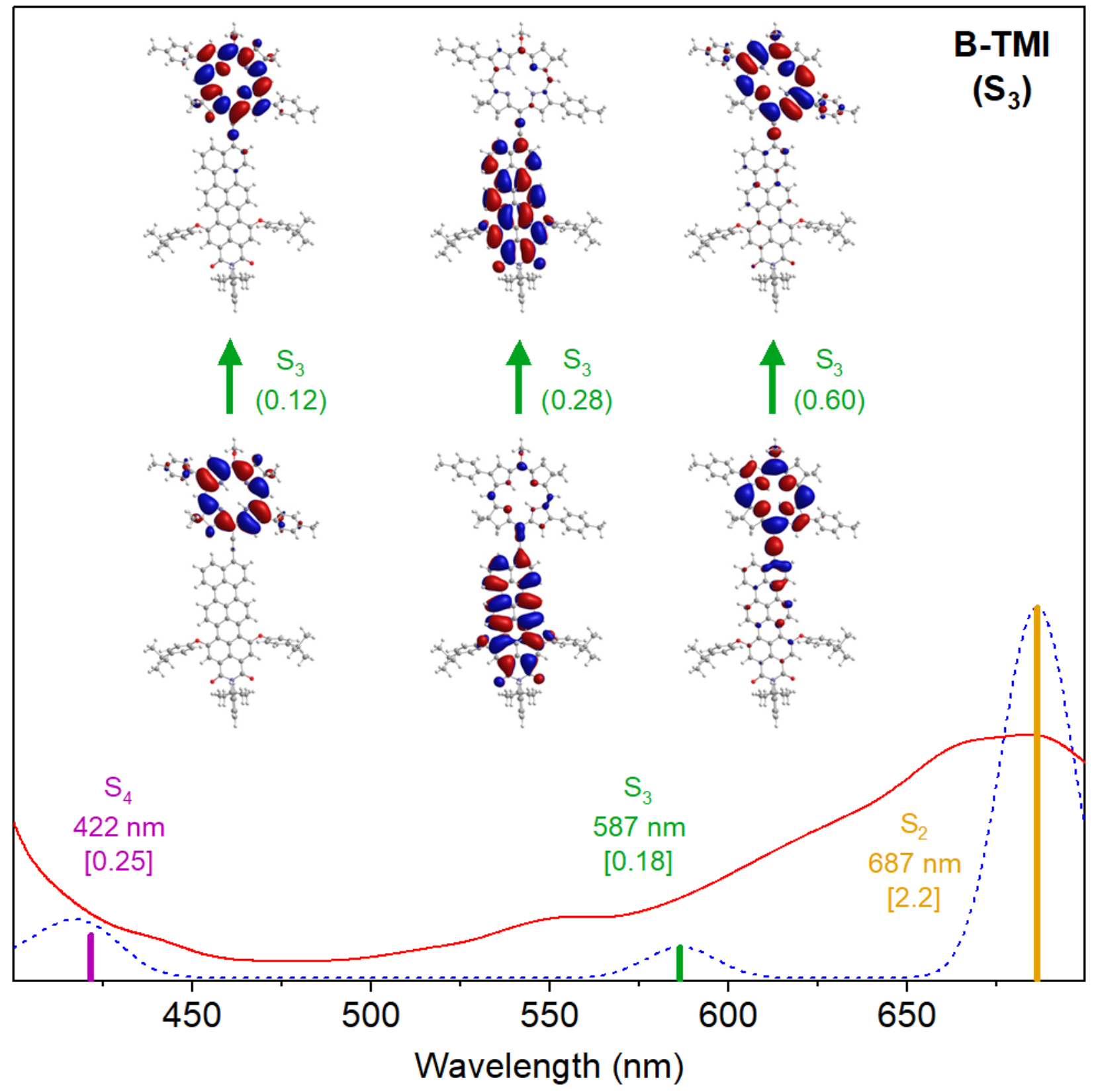

Figure S73. Comparison for dyad B-TMI of the absorption spectra in toluene that are measured (red line) or calculated via TDDFT (dashed blue lines and colored sticks) normalized at the highest peak. The calculated spectrum was shifted arbitrarily by $800 \mathrm{~cm}^{-1}$ to lower energy to better align with the measured spectrum. Features in the calculated spectra were given 10-nm Gaussian skirts. The wavelength and oscillator strength (in square brackets) of the transition from the ground state to $S_{3}$ (green) calculated from TDDFT is indicated. Also shown is the set of occupied and virtual NTOs along with the eigenvalue (weight) for the transition. 


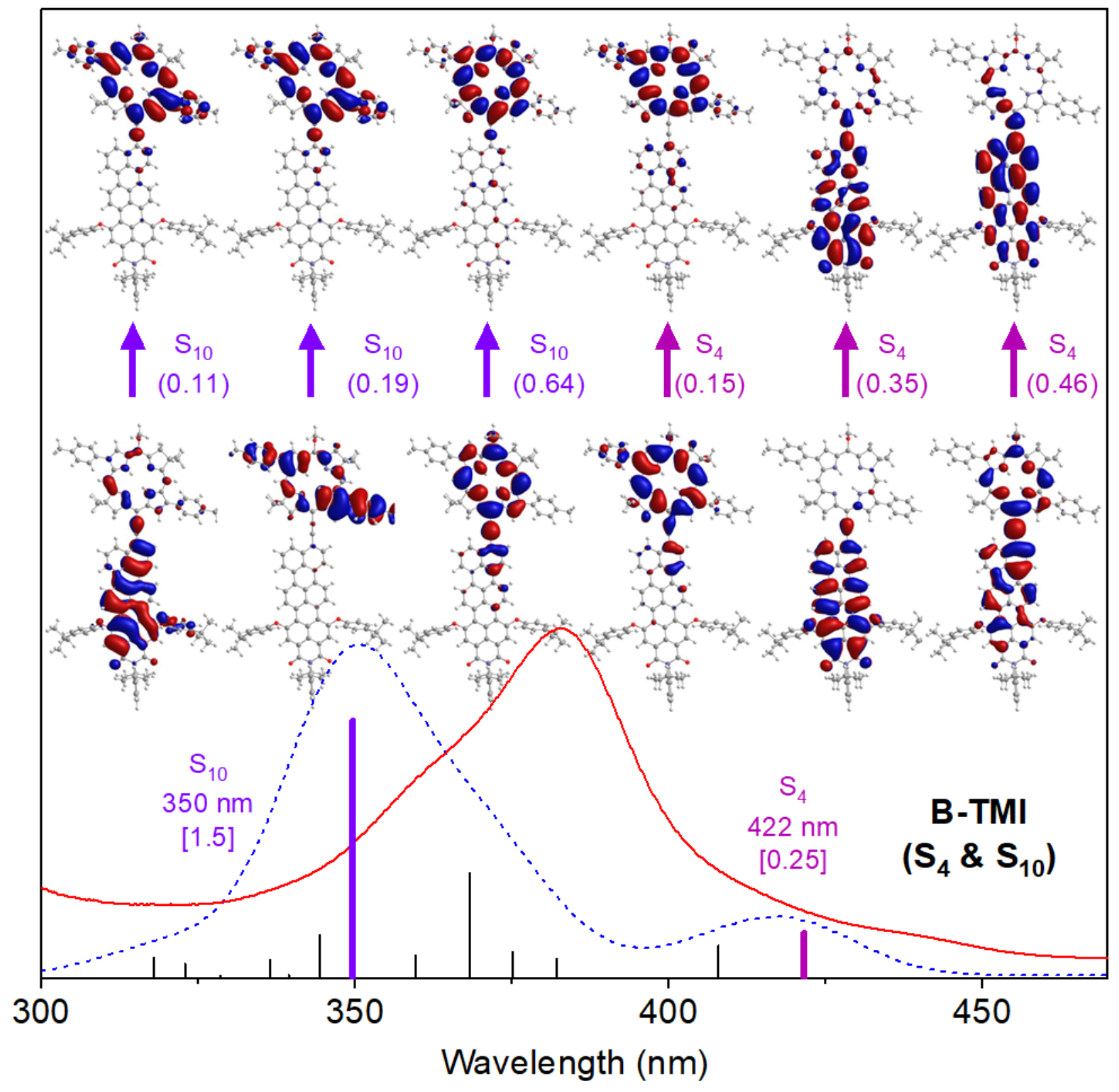

Figure S74. Comparison for dyad B-TMI of the absorption spectra in toluene that are measured (red line) or calculated via TDDFT (dashed blue lines and colored sticks) normalized at the highest peak. The calculated spectrum was shifted arbitrarily by $800 \mathrm{~cm}^{-1}$ to lower energy to better align with the measured spectrum. Features in the calculated spectra were given 10-nm Gaussian skirts. The wavelength and oscillator strength (in square brackets) of the transitions from the ground state to $S_{4}$ (mauve) and $S_{10}$ (violet) calculated from TDDFT are indicated. Also shown are the sets of occupied and virtual NTOs along with the eigenvalue (weight) for each transition. 


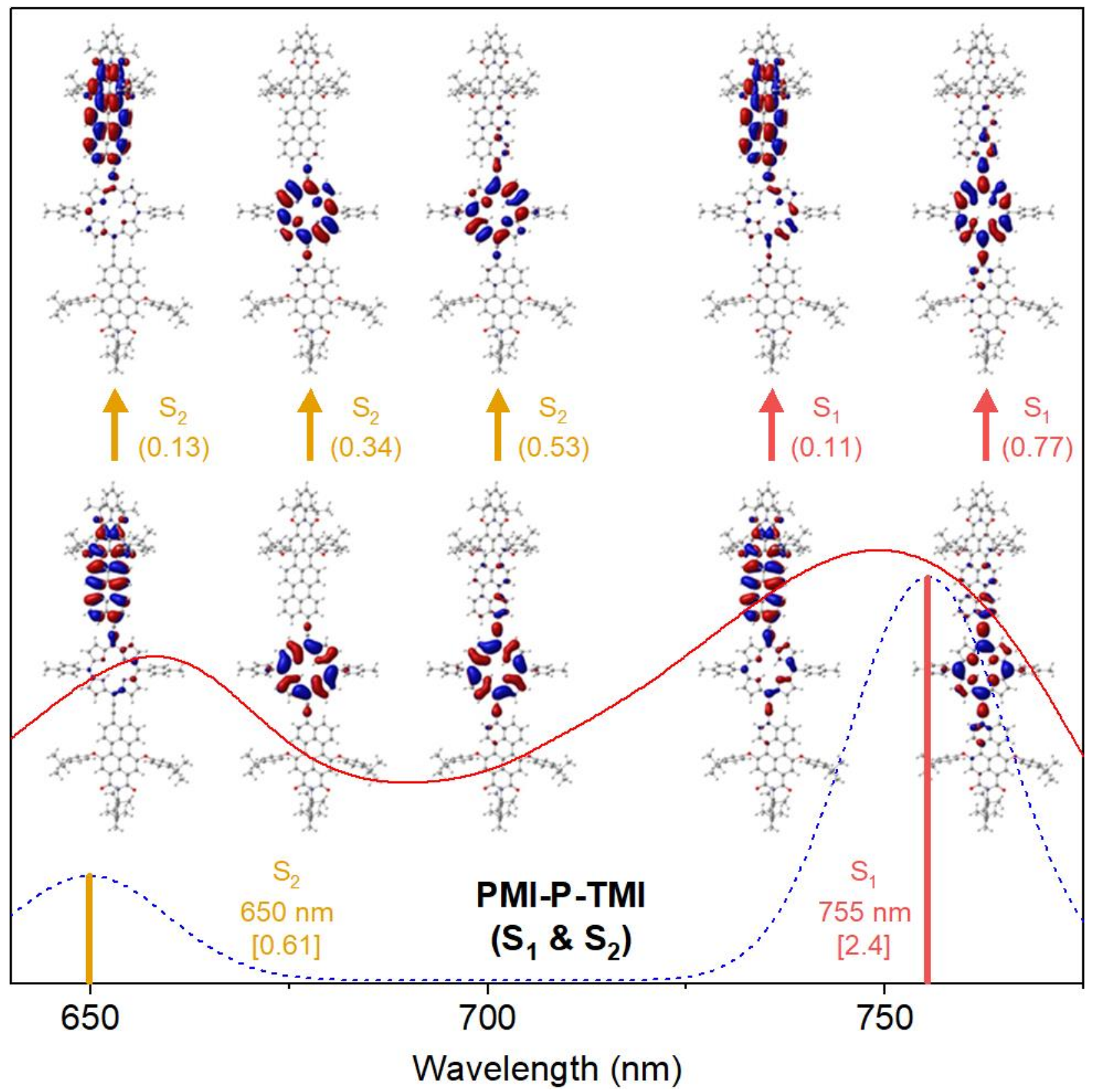

Figure S75. Comparison for triad PMI-P-TMI of the absorption spectra in toluene that are measured (red line) or calculated via TDDFT (dashed blue lines and colored sticks) normalized at the highest peak. The calculated spectrum was shifted arbitrarily by $500 \mathrm{~cm}^{-1}$ to lower energy to better align with the measured spectrum. Features in the calculated spectra were given 10-nm Gaussian skirts. The wavelength and oscillator strength (in square brackets) of the transitions from the ground state to $S_{1}$ (red) and $S_{2}$ (gold) calculated from TDDFT are indicated. Also shown are the sets of occupied and virtual NTOs along with the eigenvalue (weight) for each transition. 


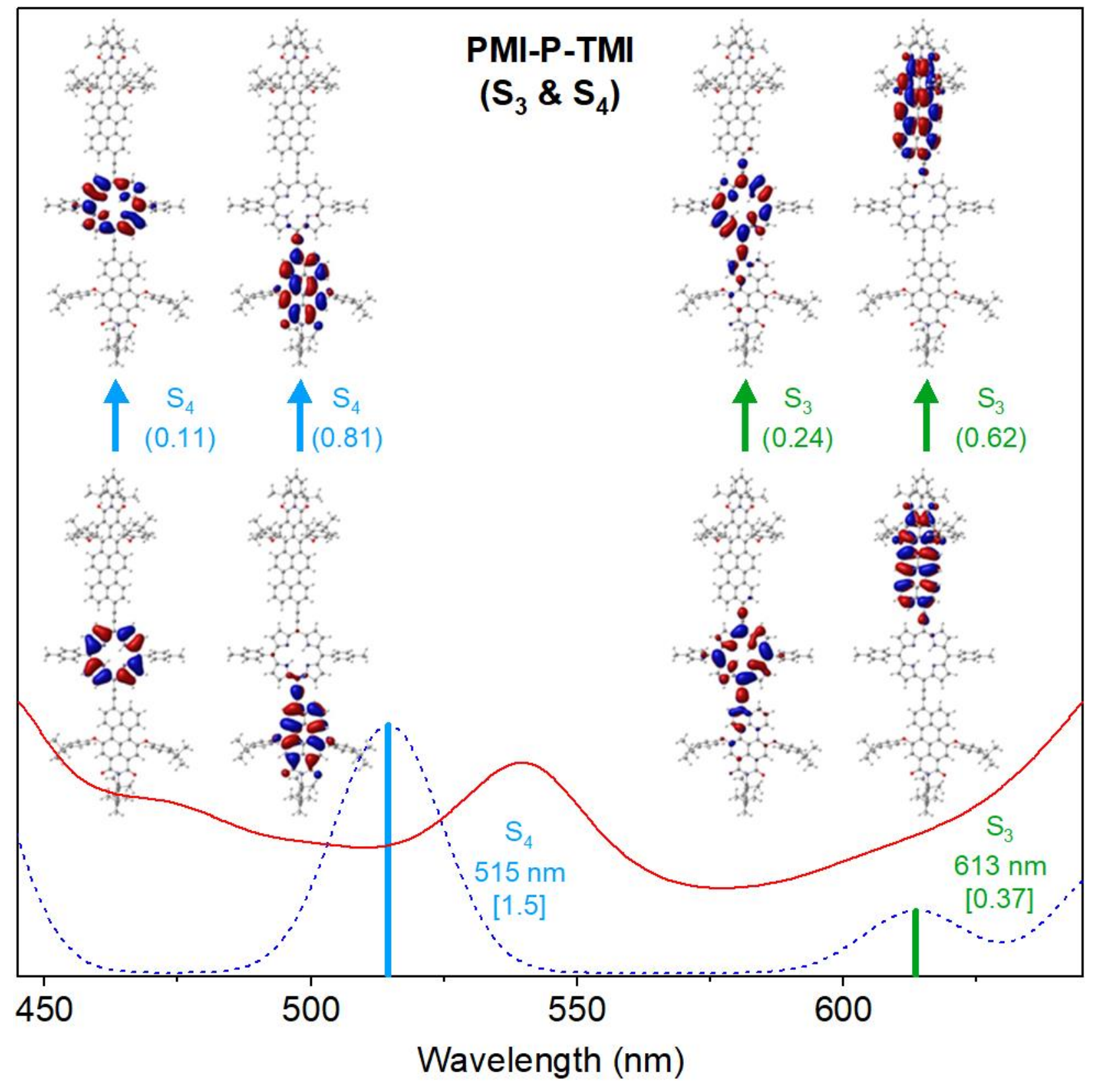

Figure S76. Comparison for triad PMI-P-TMI of the absorption spectra in toluene that are measured (red line) or calculated via TDDFT (dashed blue lines and colored sticks) normalized at the highest peak. The calculated spectrum was shifted arbitrarily by $500 \mathrm{~cm}^{-1}$ to lower energy to better align with the measured spectrum. Features in the calculated spectra were given 10-nm Gaussian skirts. The wavelength and oscillator strength (in square brackets) of the transition from the ground state to $S_{3}$ (green) and $S_{4}$ (blue) calculated from TDDFT is indicated. Also shown is the set of occupied and virtual NTOs along with the eigenvalue (weight) for the transitions. 


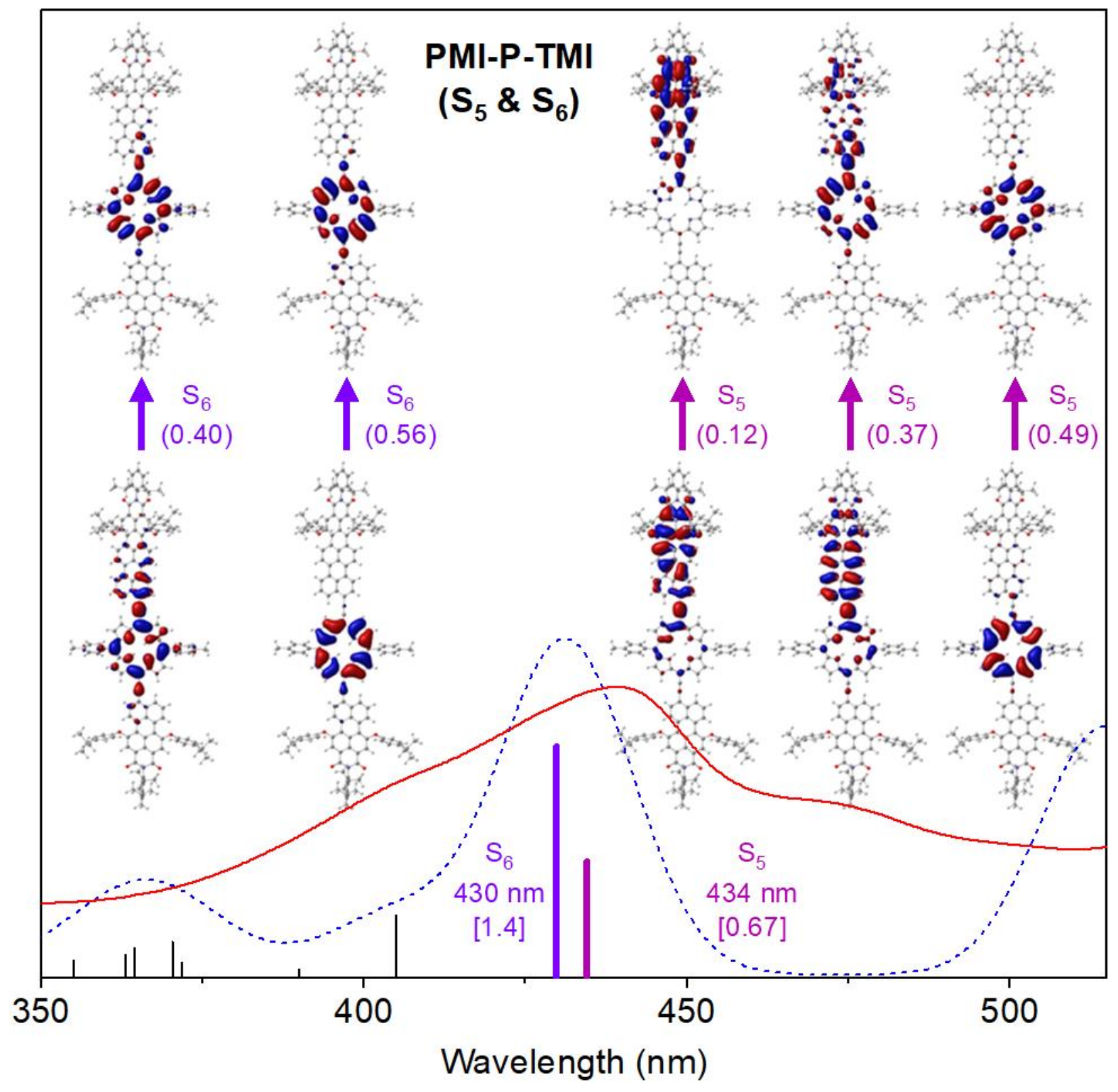

Figure S77. Comparison for triad PMI-P-TMI of the absorption spectra in toluene that are measured (red line) or calculated via TDDFT (dashed blue lines and colored sticks) normalized at the highest peak. The calculated spectrum was shifted arbitrarily by $500 \mathrm{~cm}^{-1}$ to lower energy to better align with the measured spectrum. Features in the calculated spectra were given 10-nm Gaussian skirts. The wavelength and oscillator strength (in square brackets) of the transitions from the ground state to $\mathrm{S}_{5}$ (mauve) and $\mathrm{S}_{6}$ (violet) calculated from TDDFT are indicated. Also shown are the sets of occupied and virtual NTOs along with the eigenvalue (weight) for each transition. 


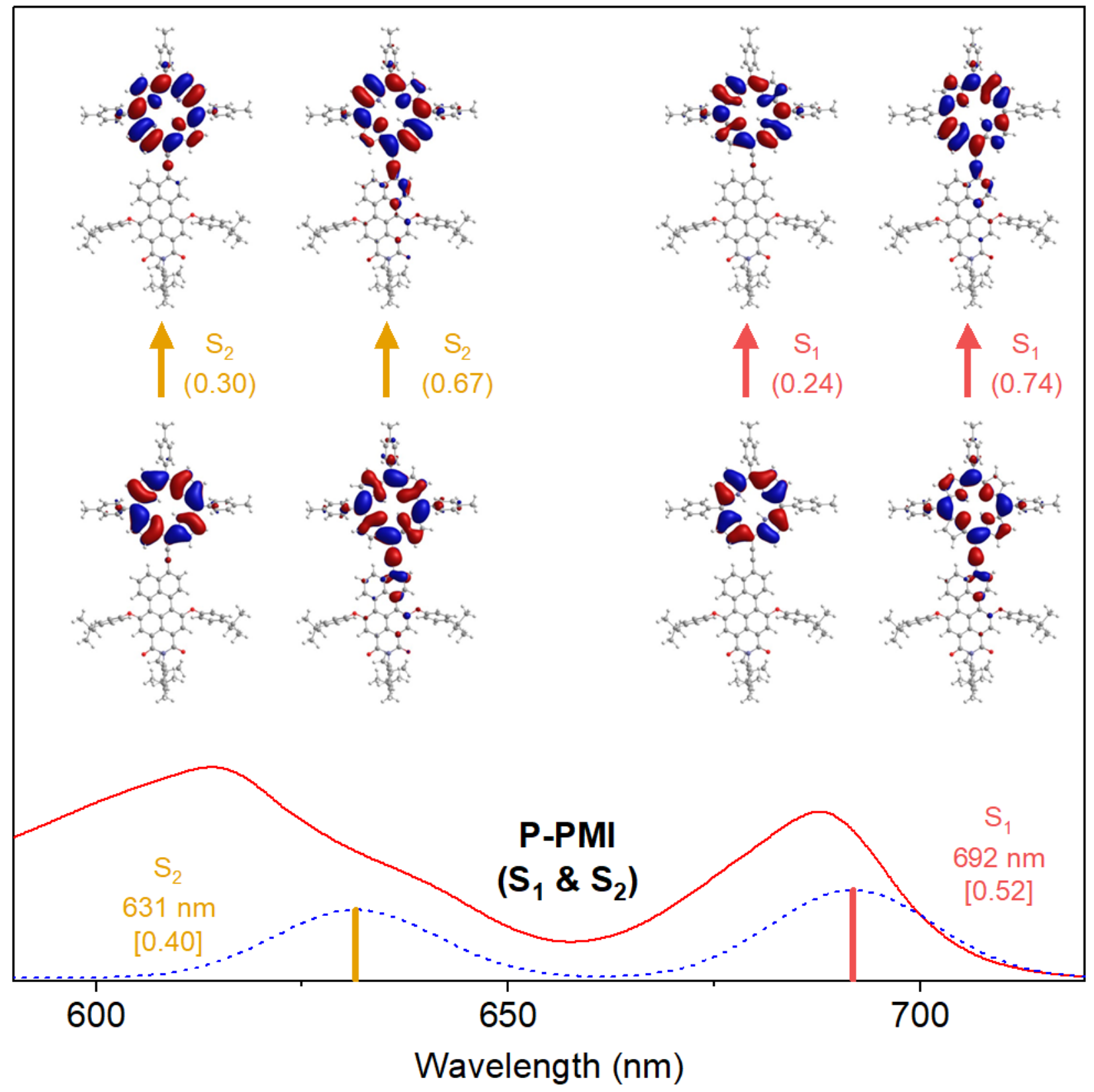

Figure S78. Comparison for dyad P-PMI of the absorption spectra in toluene that are measured (red line) or calculated via TDDFT (dashed blue lines and colored sticks) normalized at the highest peak. The calculated spectrum was shifted arbitrarily by $850 \mathrm{~cm}^{-1}$ to lower energy to better align with the measured spectrum. Features in the calculated spectra were given 10-nm Gaussian skirts. The wavelength and oscillator strength (in square brackets) of the transitions from the ground state to $S_{1}$ (red) and $S_{2}$ (gold) calculated from TDDFT are indicated. Also shown are the sets of occupied and virtual NTOs along with the eigenvalue (weight) for each transition. 


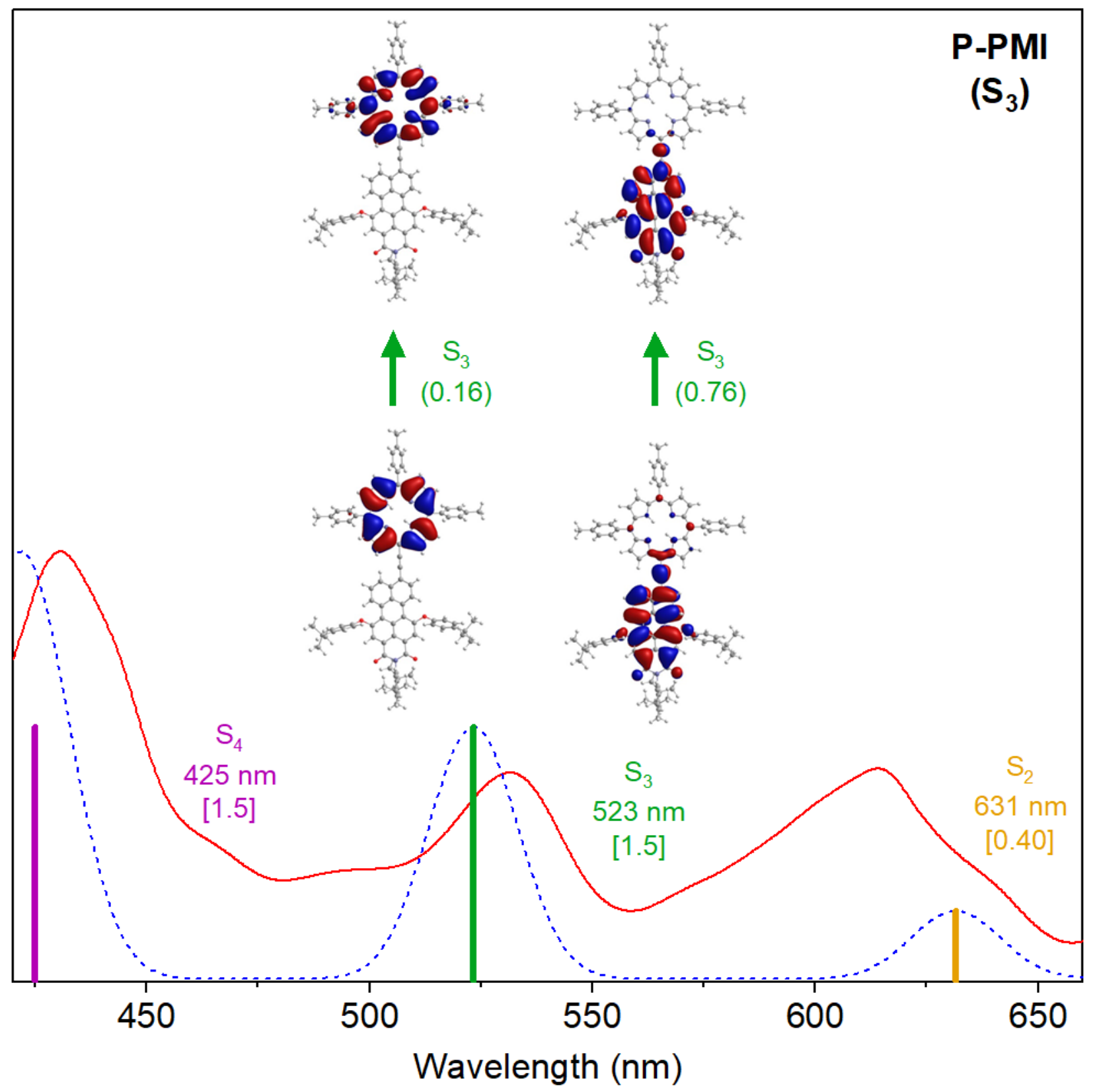

Figure S79. Comparison for dyad P-PMI of the absorption spectra in toluene that are measured (red line) or calculated via TDDFT (dashed blue lines and colored sticks) normalized at the highest peak. The calculated spectrum was shifted arbitrarily by $850 \mathrm{~cm}^{-1}$ to lower energy to better align with the measured spectrum. Features in the calculated spectra were given 10-nm Gaussian skirts. The wavelength and oscillator strength (in square brackets) of the transitions from the ground state to $\mathrm{S}_{3}$ (green) calculated from TDDFT are indicated. Also shown are the sets of occupied and virtual NTOs along with the eigenvalue (weight) for each transition. 


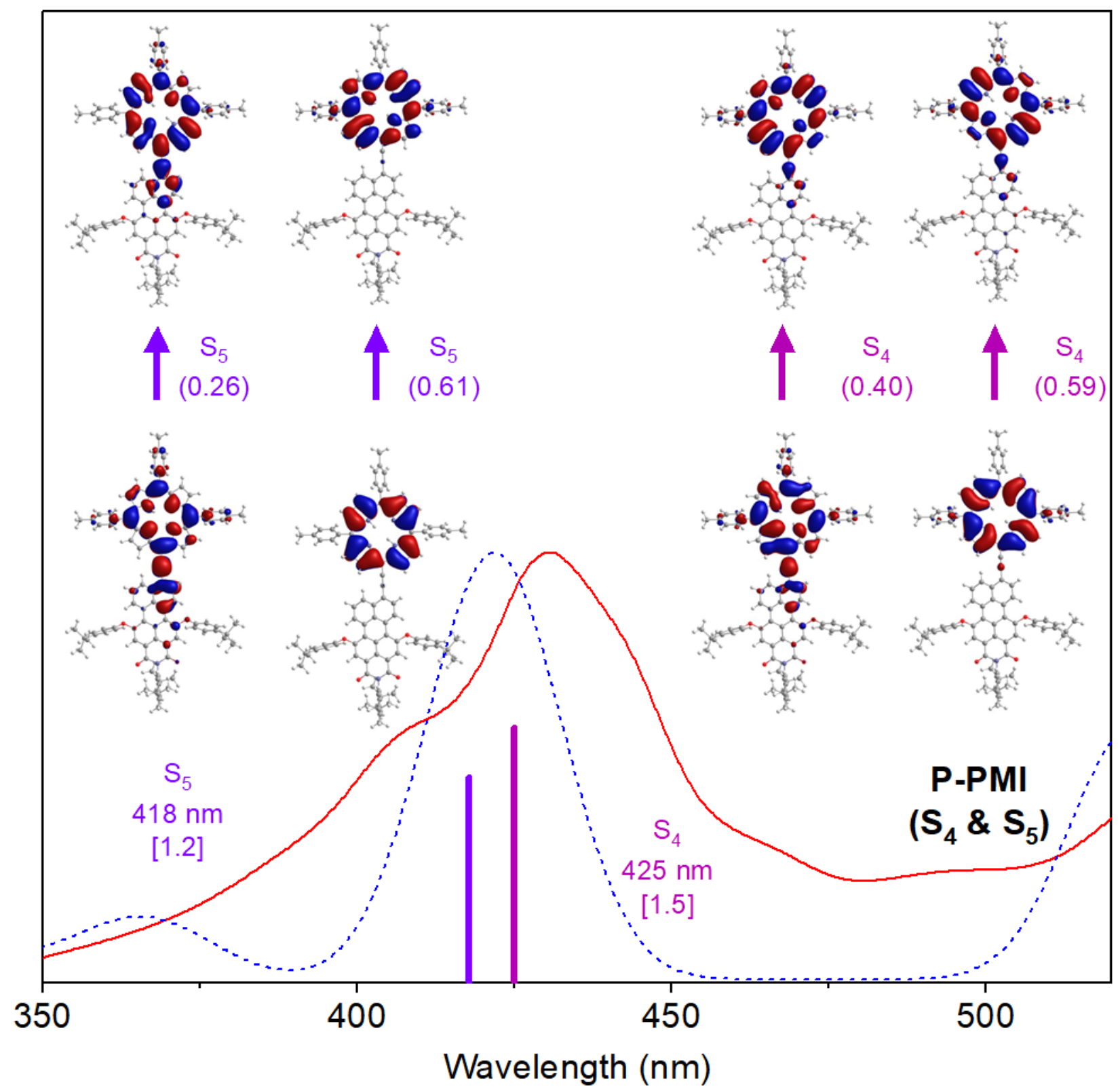

Figure S80. Comparison for dyad P-PMI of the absorption spectra in toluene that are measured (red line) or calculated via TDDFT (dashed blue lines and colored sticks) normalized at the highest peak. The calculated spectrum was shifted arbitrarily by $850 \mathrm{~cm}^{-1}$ to lower energy to better align with the measured spectrum. Features in the calculated spectra were given 10-nm Gaussian skirts. The wavelength and oscillator strength (in square brackets) of the transitions from the ground state to $\mathrm{S}_{4}$ (mauve) and $\mathrm{S}_{5}$ (violet) calculated from TDDFT are indicated. Also shown are the sets of occupied and virtual NTOs along with the eigenvalue (weight) for each transition. 


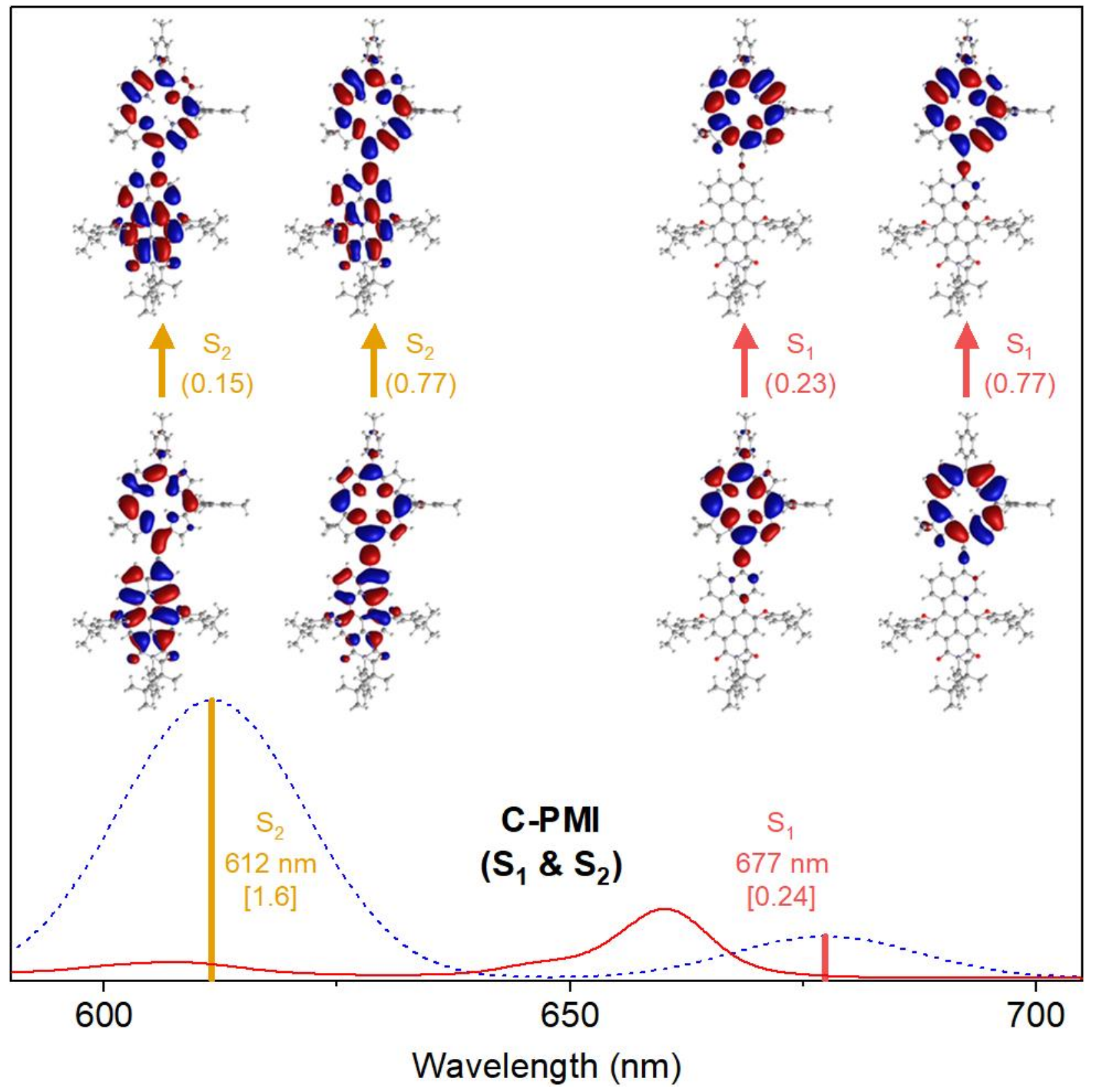

Figure S81. Comparison for dyad C-PMI of the absorption spectra in toluene that are measured (red line) or calculated via TDDFT (dashed blue lines and colored sticks) normalized at the highest peak. The calculated spectrum was shifted arbitrarily by $1400 \mathrm{~cm}^{-1}$ to lower energy to better align with the measured spectrum. Features in the calculated spectra were given 10-nm Gaussian skirts. The wavelength and oscillator strength (in square brackets) of the transitions from the ground state to $S_{1}$ (red) and $S_{2}$ (gold) calculated from TDDFT are indicated. Also shown are the sets of occupied and virtual NTOs along with the eigenvalue (weight) for each transition. 


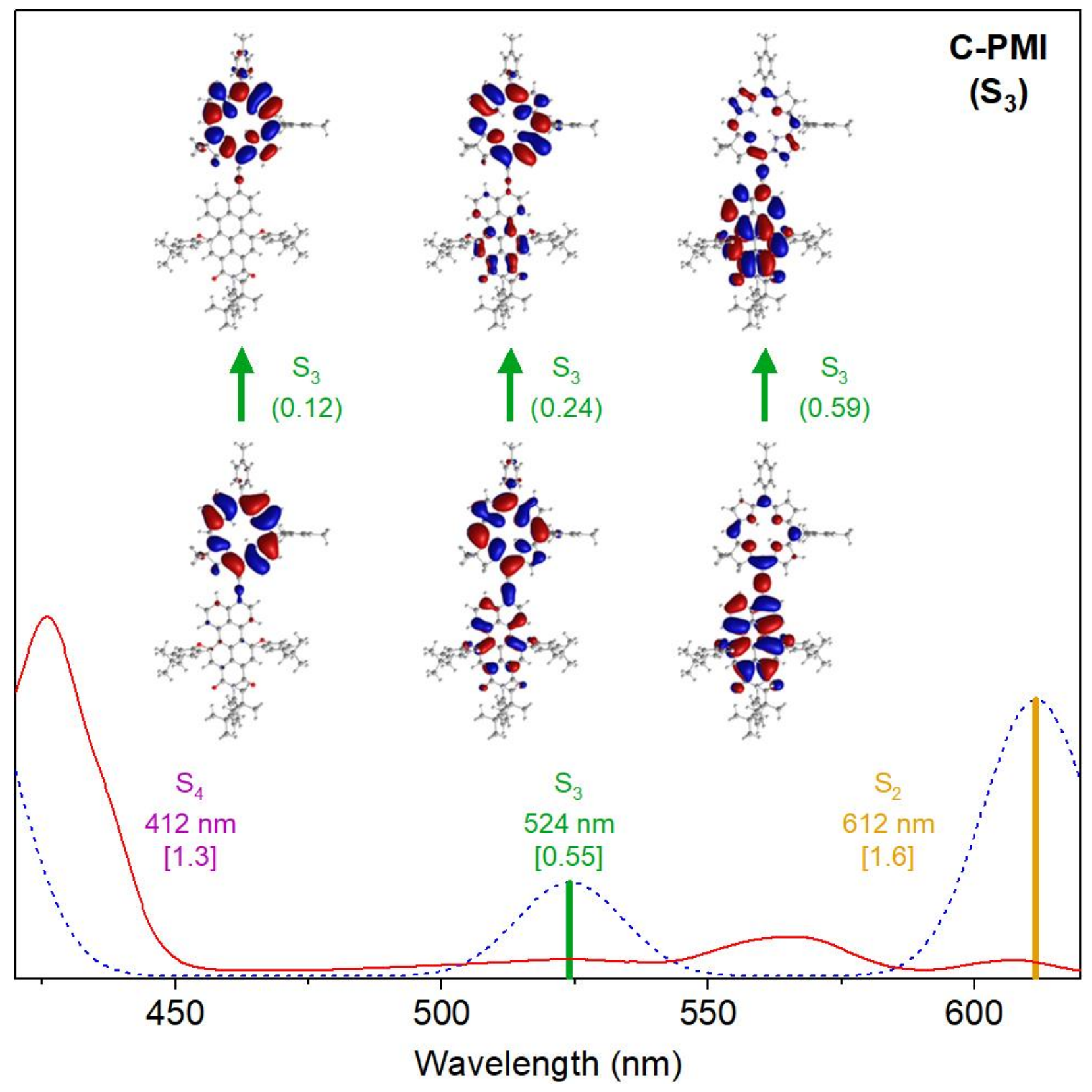

Figure S82. Comparison for dyad C-PMI of the absorption spectra in toluene that are measured (red line) or calculated via TDDFT (dashed blue lines and colored sticks) normalized at the highest peak. The calculated spectrum was shifted arbitrarily by $1400 \mathrm{~cm}^{-1}$ to lower energy to better align with the measured spectrum. Features in the calculated spectra were given 10-nm Gaussian skirts. The wavelength and oscillator strength (in square brackets) of the transitions from the ground state to $S_{3}$ (green) and calculated from TDDFT are indicated. Also shown are the sets of occupied and virtual NTOs along with the eigenvalue (weight) for each transition. 


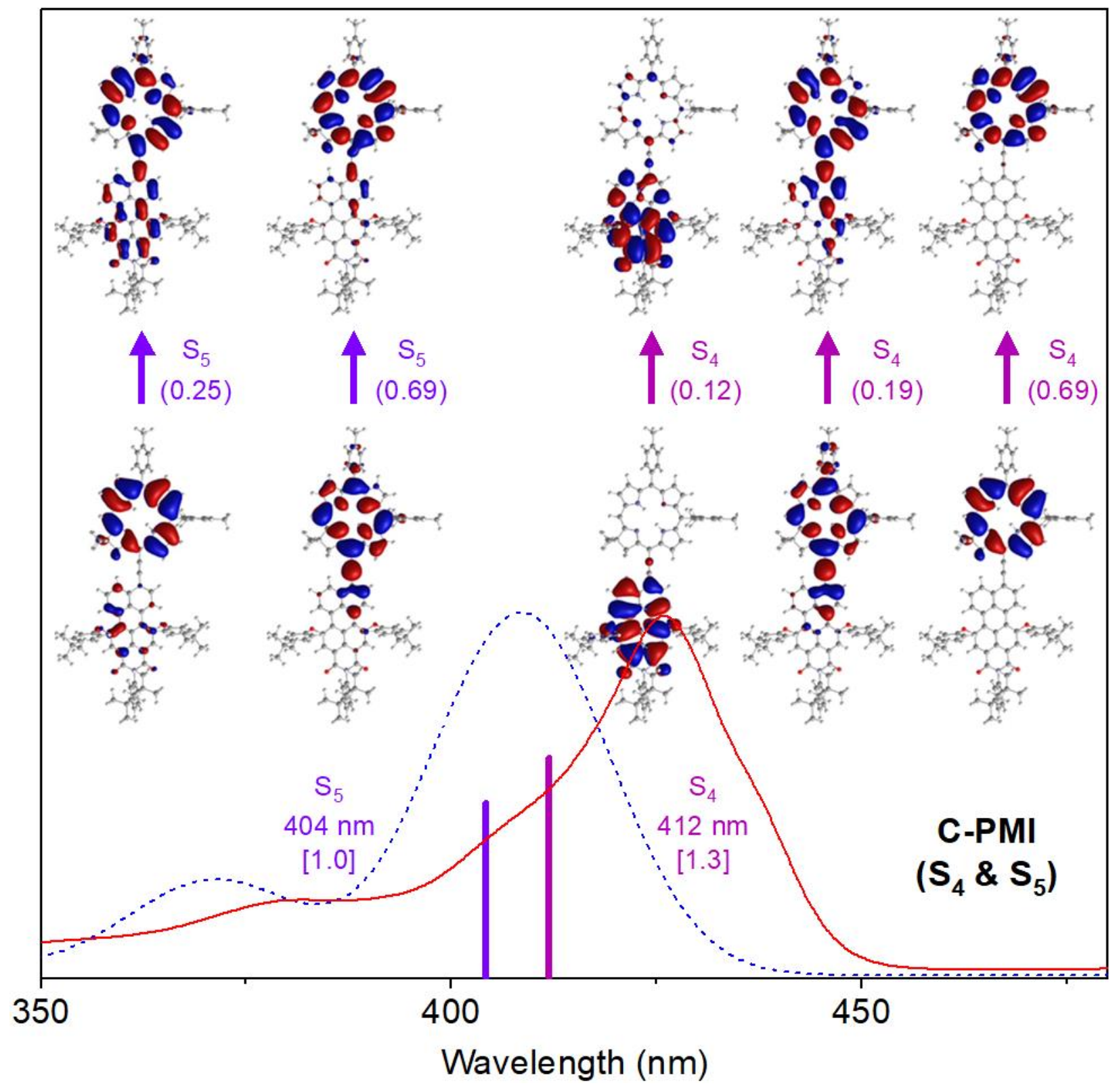

Figure S83. Comparison for dyad C-PMI of the absorption spectra in toluene that are measured (red line) or calculated via TDDFT (dashed blue lines and colored sticks) normalized at the highest peak. The calculated spectrum was shifted arbitrarily by $1400 \mathrm{~cm}^{-1}$ to lower energy to better align with the measured spectrum. Features in the calculated spectra were given 10-nm Gaussian skirts. The wavelength and oscillator strength (in square brackets) of the transitions from the ground state to $S_{4}$ (mauve) and $S_{5}$ (violet) calculated from TDDFT are indicated. Also shown are the sets of occupied and virtual NTOs along with the eigenvalue (weight) for each transition. 


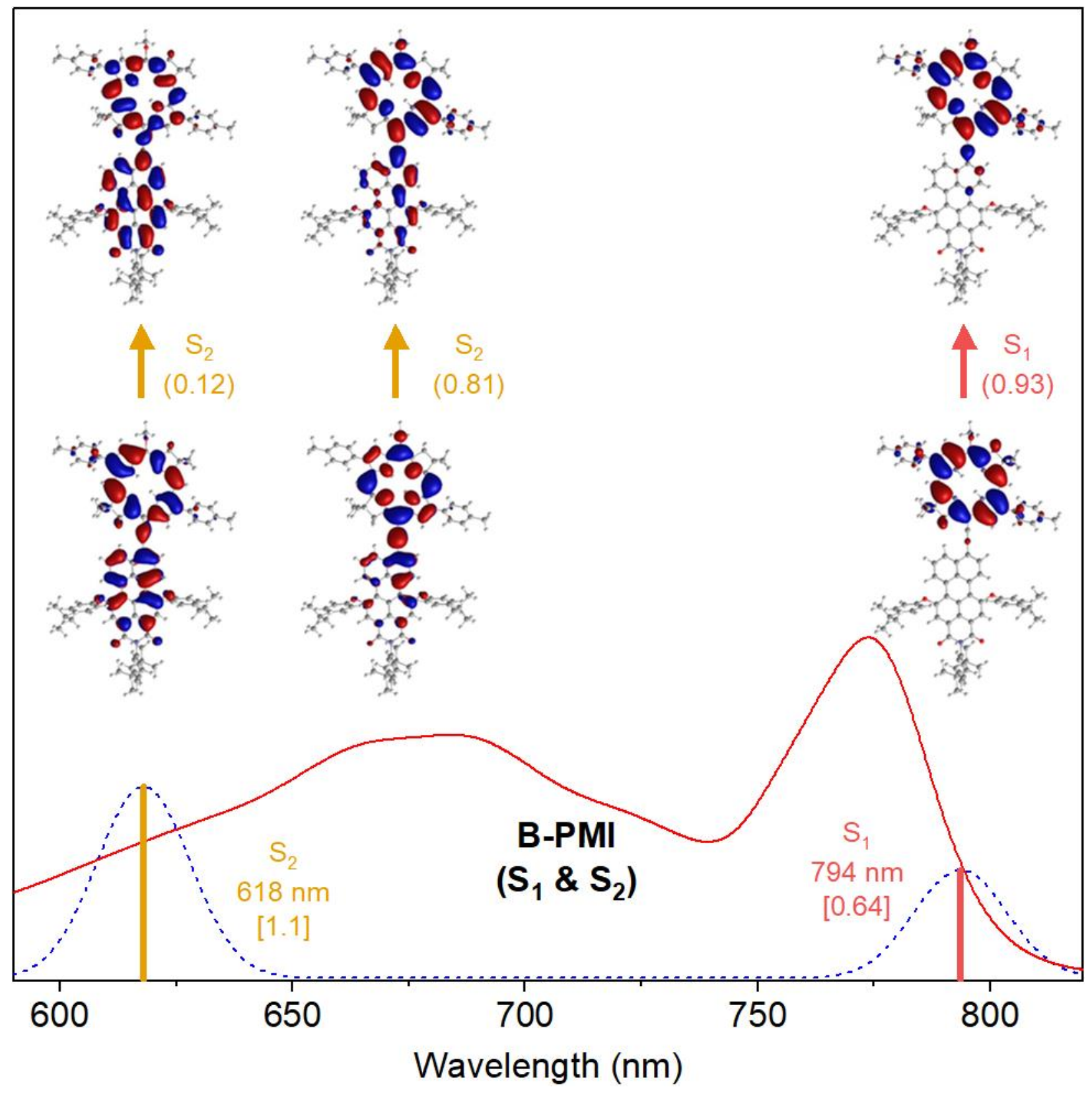

Figure S84. Comparison for dyad B-PMI of the absorption spectra in toluene that are measured (red line) or calculated via TDDFT (dashed blue lines and colored sticks) normalized at the highest peak. The calculated spectrum was shifted arbitrarily by $500 \mathrm{~cm}^{-1}$ to lower energy to better align with the measured spectrum. Features in the calculated spectra were given 10-nm Gaussian skirts. The wavelength and oscillator strength (in square brackets) of the transitions from the ground state to $S_{1}$ (red) and $S_{2}$ (gold) calculated from TDDFT are indicated. Also shown are the sets of occupied and virtual NTOs along with the eigenvalue (weight) for each transition. 


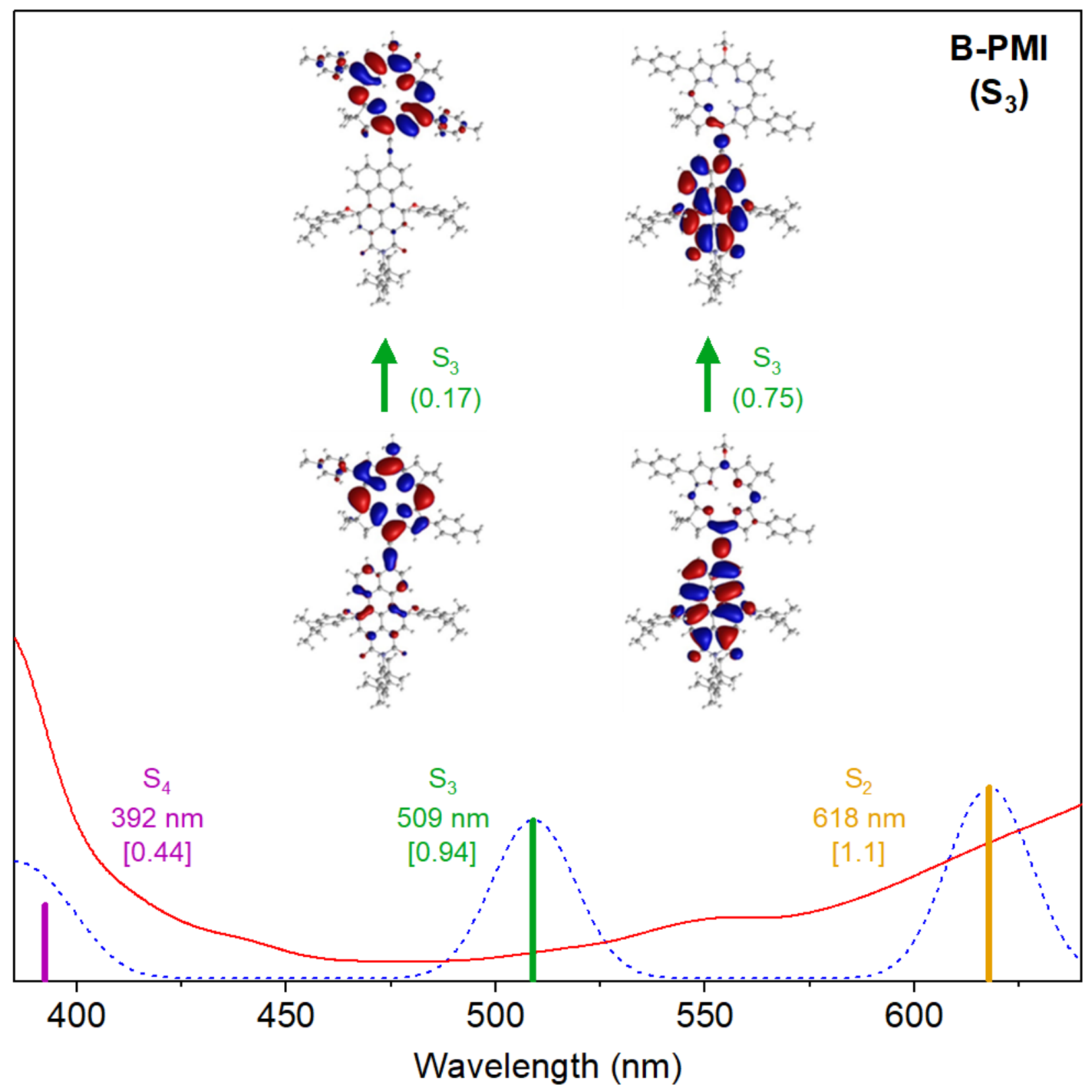

Figure S85. Comparison for dyad B-PMI of the absorption spectra in toluene that are measured (red line) or calculated via TDDFT (dashed blue lines and colored sticks) normalized at the highest peak. The calculated spectrum was shifted arbitrarily by $500 \mathrm{~cm}^{-1}$ to lower energy to better align with the measured spectrum. Features in the calculated spectra were given 10-nm Gaussian skirts. The wavelength and oscillator strength (in square brackets) of the transitions from the ground state to $\mathrm{S}_{3}$ (green) calculated from TDDFT are indicated. Also shown are the sets of occupied and virtual NTOs along with the eigenvalue (weight) for each transition. 


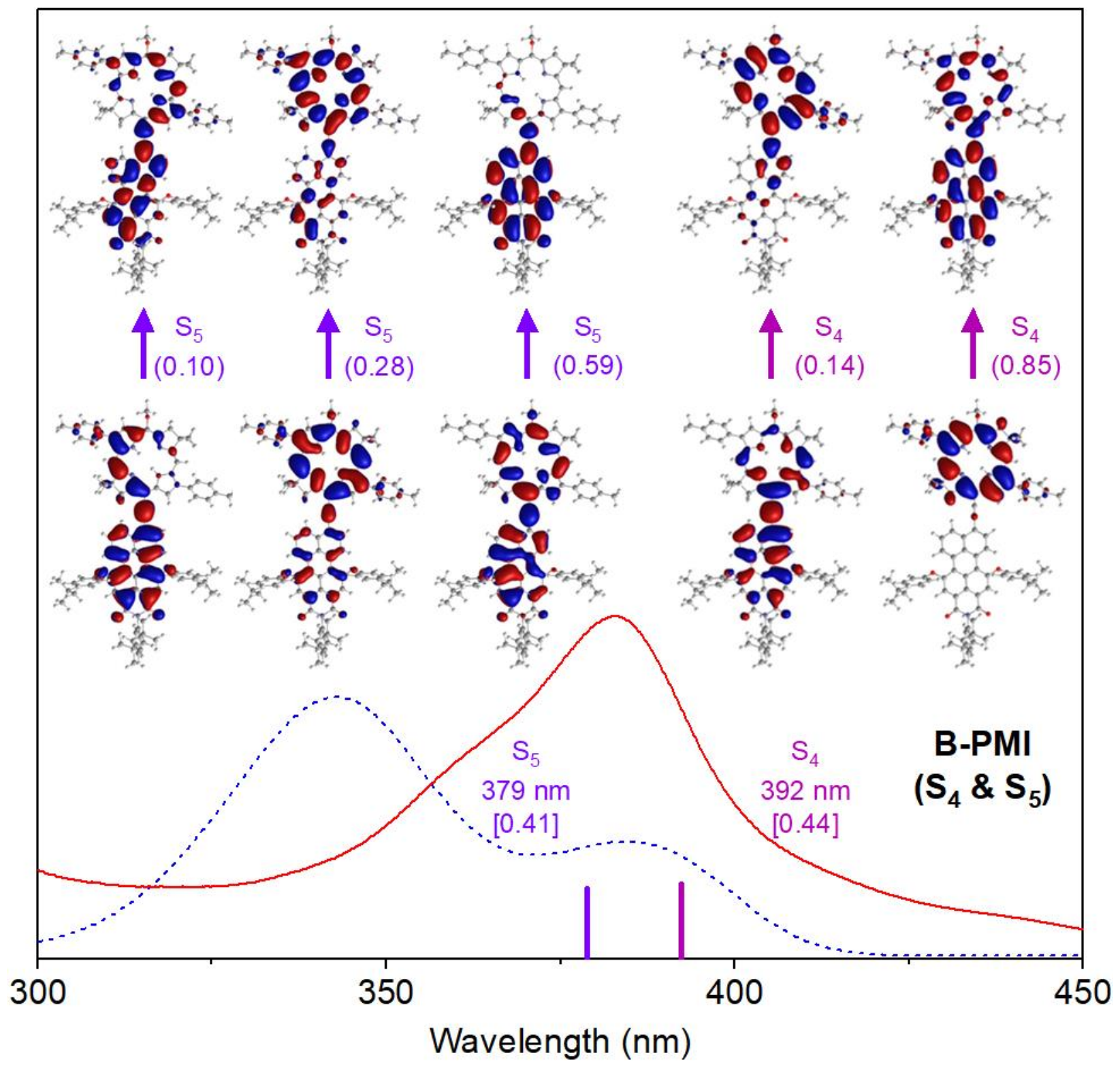

Figure S86. Comparison for dyad B-PMI of the absorption spectra in toluene that are measured (red line) or calculated via TDDFT (dashed blue lines and colored sticks) normalized at the highest peak. The calculated spectrum was shifted arbitrarily by $500 \mathrm{~cm}^{-1}$ to lower energy to better align with the measured spectrum. Features in the calculated spectra were given 10-nm Gaussian skirts. The wavelength and oscillator strength (in square brackets) of the transitions from the ground state to $\mathrm{S}_{4}$ (mauve) and $\mathrm{S}_{5}$ (violet) calculated from TDDFT are indicated. Also shown are the sets of occupied and virtual NTOs along with the eigenvalue (weight) for each transition. 


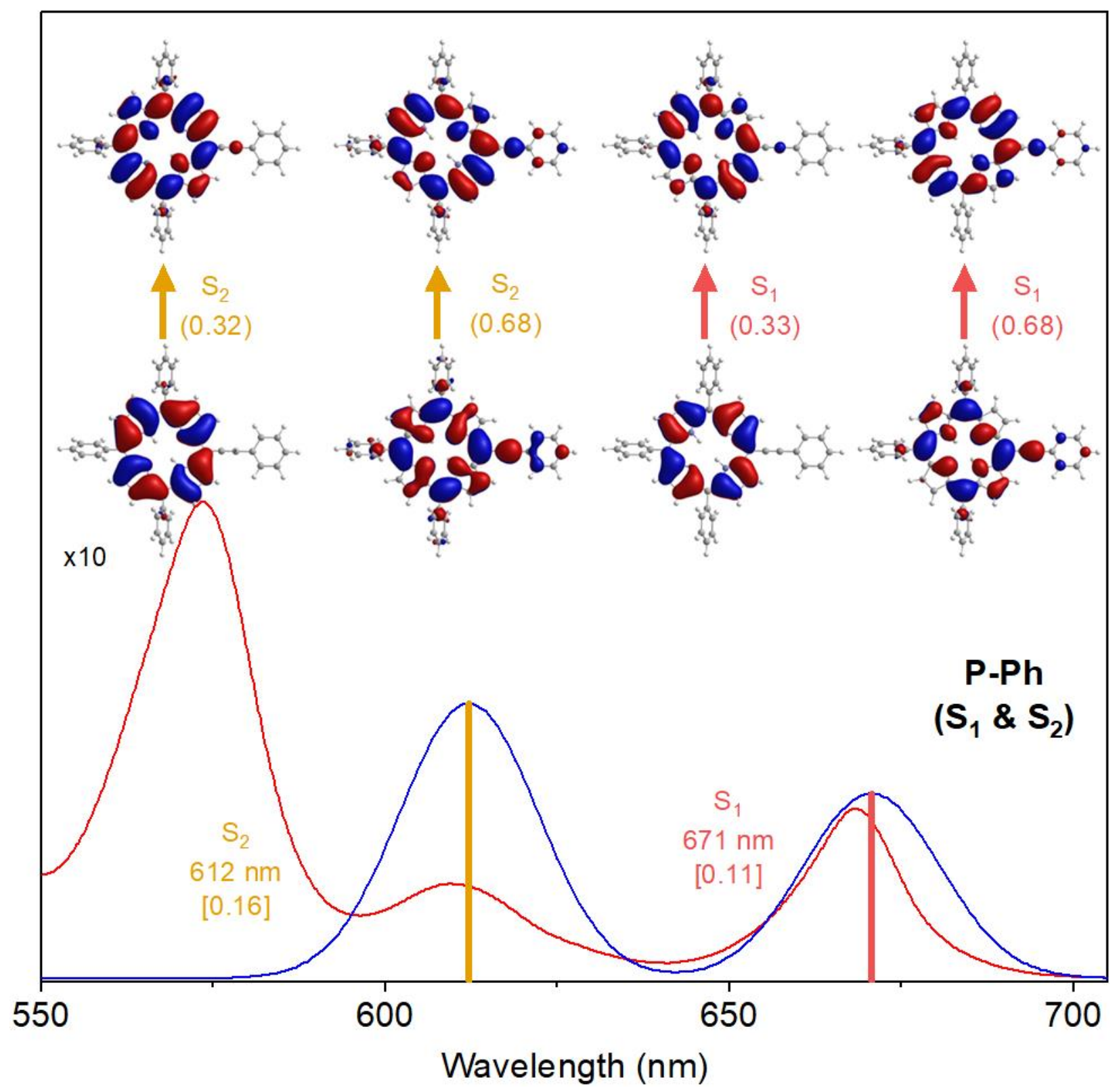

Figure S87 (was 79). Comparison for benchmark P-Ph of the absorption spectra in toluene that are measured (red line) or calculated via TDDFT (dashed blue lines and colored sticks) normalized at the highest peak. The calculated spectrum was shifted arbitrarily by $700 \mathrm{~cm}^{-1}$ to lower energy to better align with the measured spectrum. Features in the calculated spectra were given 10-nm Gaussian skirts. The wavelength and oscillator strength (in square brackets) of the transitions from the ground state to $S_{1}$ (red) and $S_{2}$ (gold) calculated from TDDFT are indicated. Also shown are the sets of occupied and virtual NTOs along with the eigenvalue (weight) for each transition. 


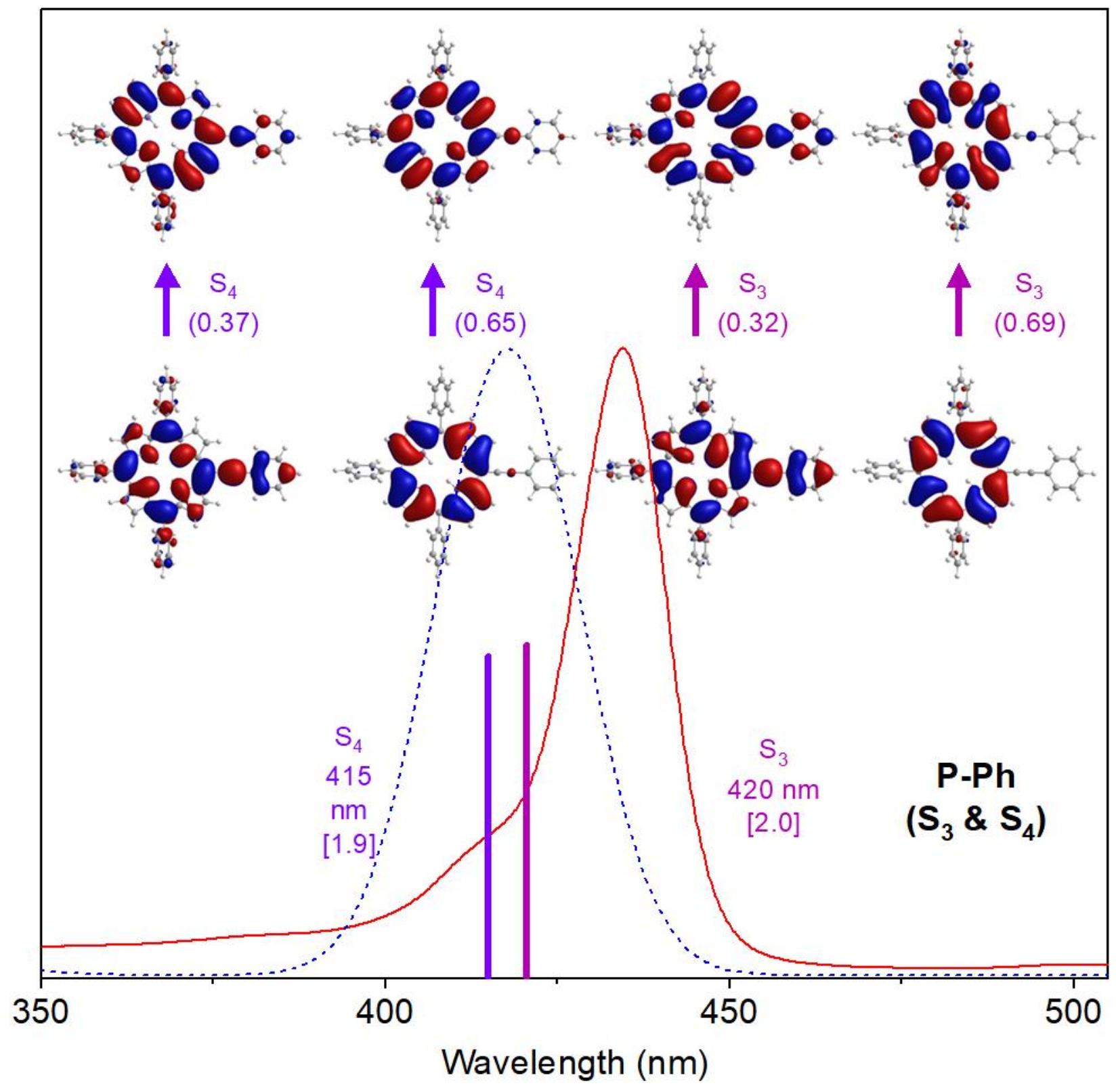

Figure S88. Comparison for benchmark P-Ph of the absorption spectra in toluene that are measured (red line) or calculated via TDDFT (dashed blue lines and colored sticks) normalized at the highest peak. The calculated spectrum was shifted arbitrarily by $700 \mathrm{~cm}^{-1}$ to lower energy to better align with the measured spectrum. Features in the calculated spectra were given 10-nm Gaussian skirts. The wavelength and oscillator strength (in square brackets) of the transitions from the ground state to $S_{3}$ (mauve) and $S_{4}$ (violet) calculated from TDDFT are indicated. Also shown are the sets of occupied and virtual NTOs along with the eigenvalue (weight) for each transition. 


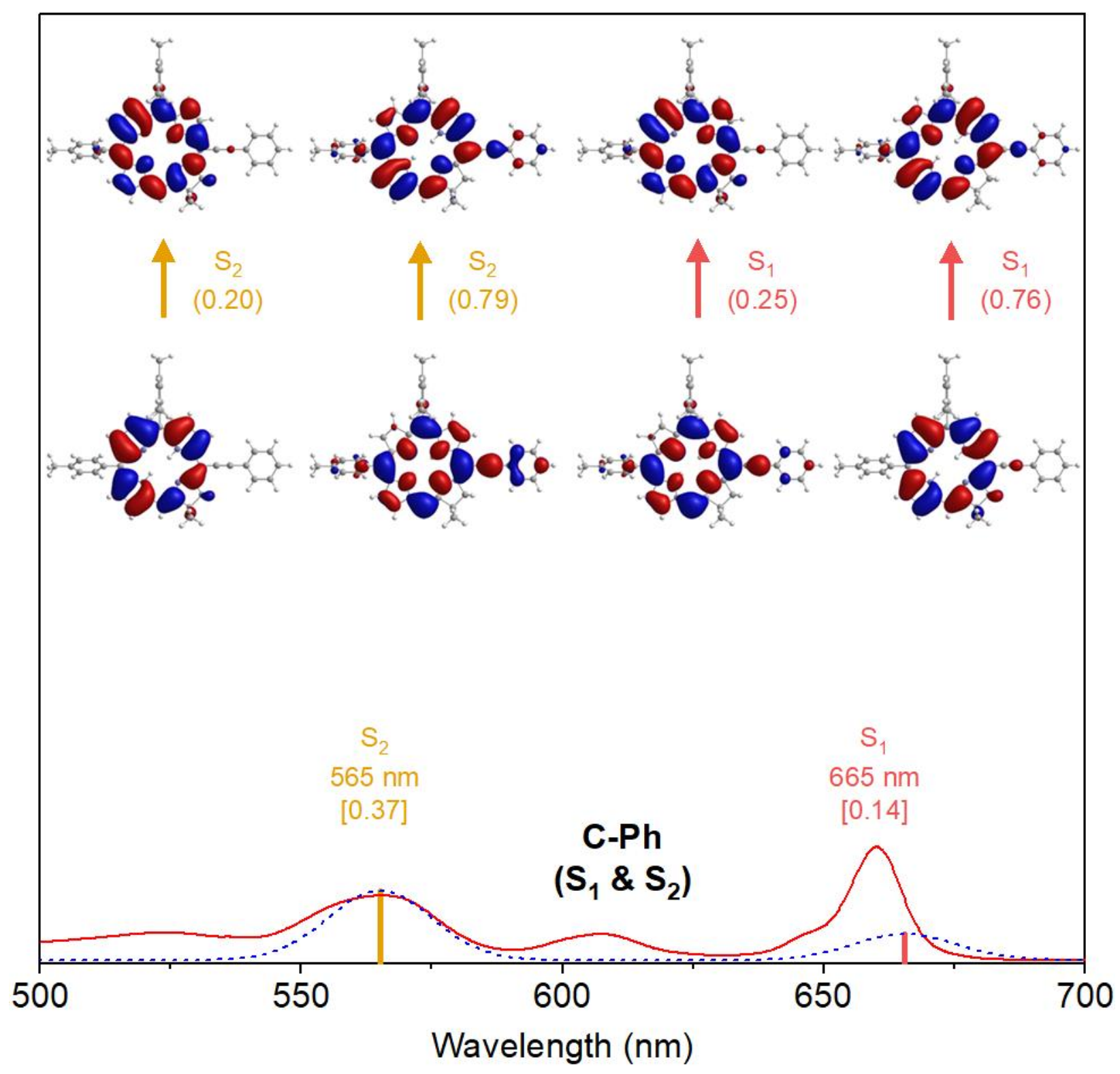

Figure S89. Comparison for benchmark C-Ph of the absorption spectra in toluene that are measured (red line) or calculated via TDDFT (dashed blue lines and colored sticks) normalized at the highest peak. The calculated spectrum was shifted arbitrarily by $1200 \mathrm{~cm}^{-1}$ to lower energy to better align with the measured spectrum. Features in the calculated spectra were given 10-nm Gaussian skirts. The wavelength and oscillator strength (in square brackets) of the transitions from the ground state to $S_{1}$ (red) and $S_{2}$ (gold) calculated from TDDFT are indicated. Also shown are the sets of occupied and virtual NTOs along with the eigenvalue (weight) for each transition. 


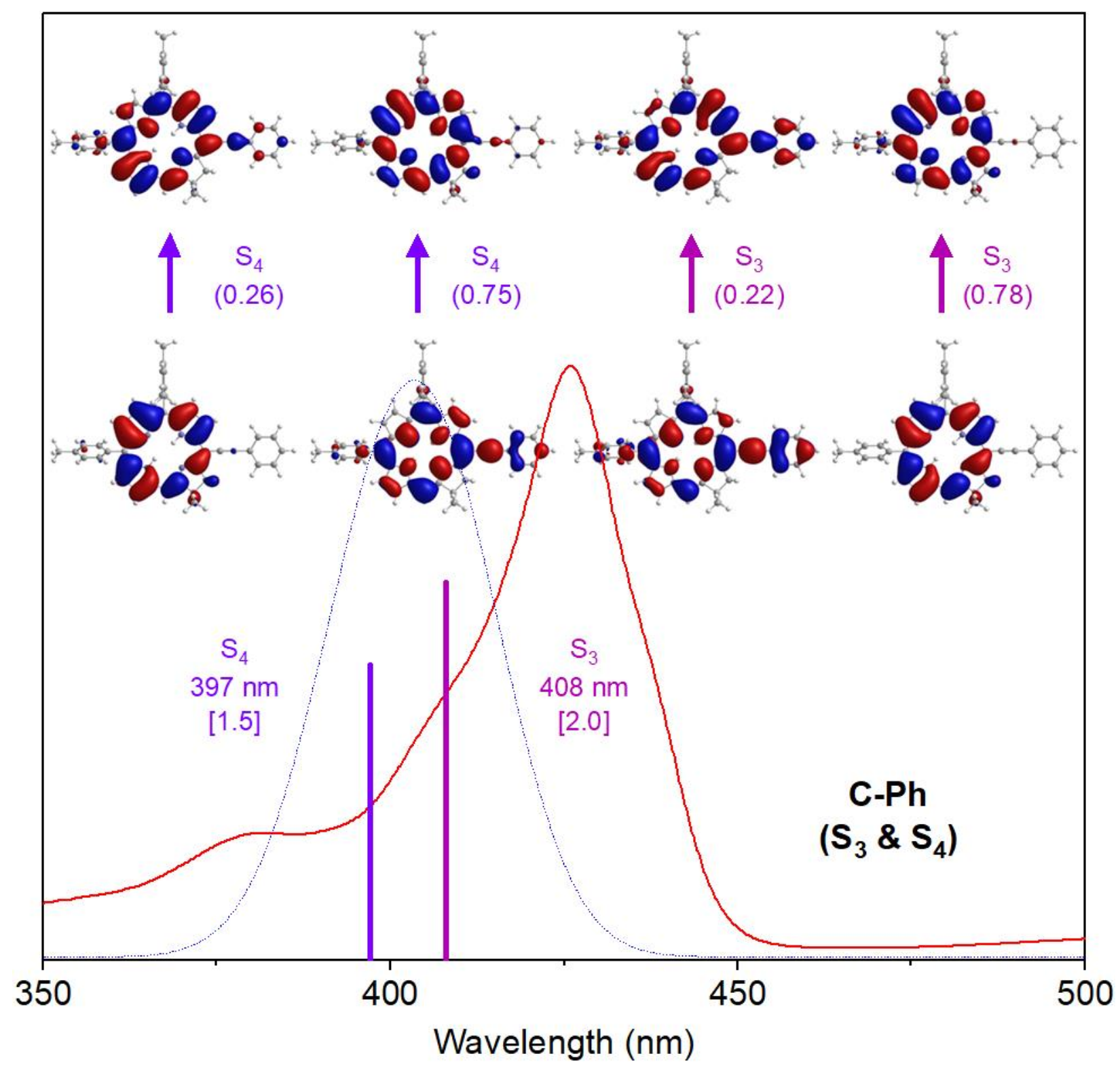

Figure S90. Comparison for benchmark C-Ph of the absorption spectra in toluene that are measured (red line) or calculated via TDDFT (dashed blue lines and colored sticks) normalized at the highest peak. The calculated spectrum was shifted arbitrarily by $1200 \mathrm{~cm}^{-1}$ to lower energy to better align with the measured spectrum. Features in the calculated spectra were given 10-nm Gaussian skirts. The wavelength and oscillator strength (in square brackets) of the transitions from the ground state to $\mathrm{S}_{3}$ (mauve) and $\mathrm{S}_{4}$ (violet) calculated from TDDFT are indicated. Also shown are the sets of occupied and virtual NTOs along with the eigenvalue (weight) for each transition. 


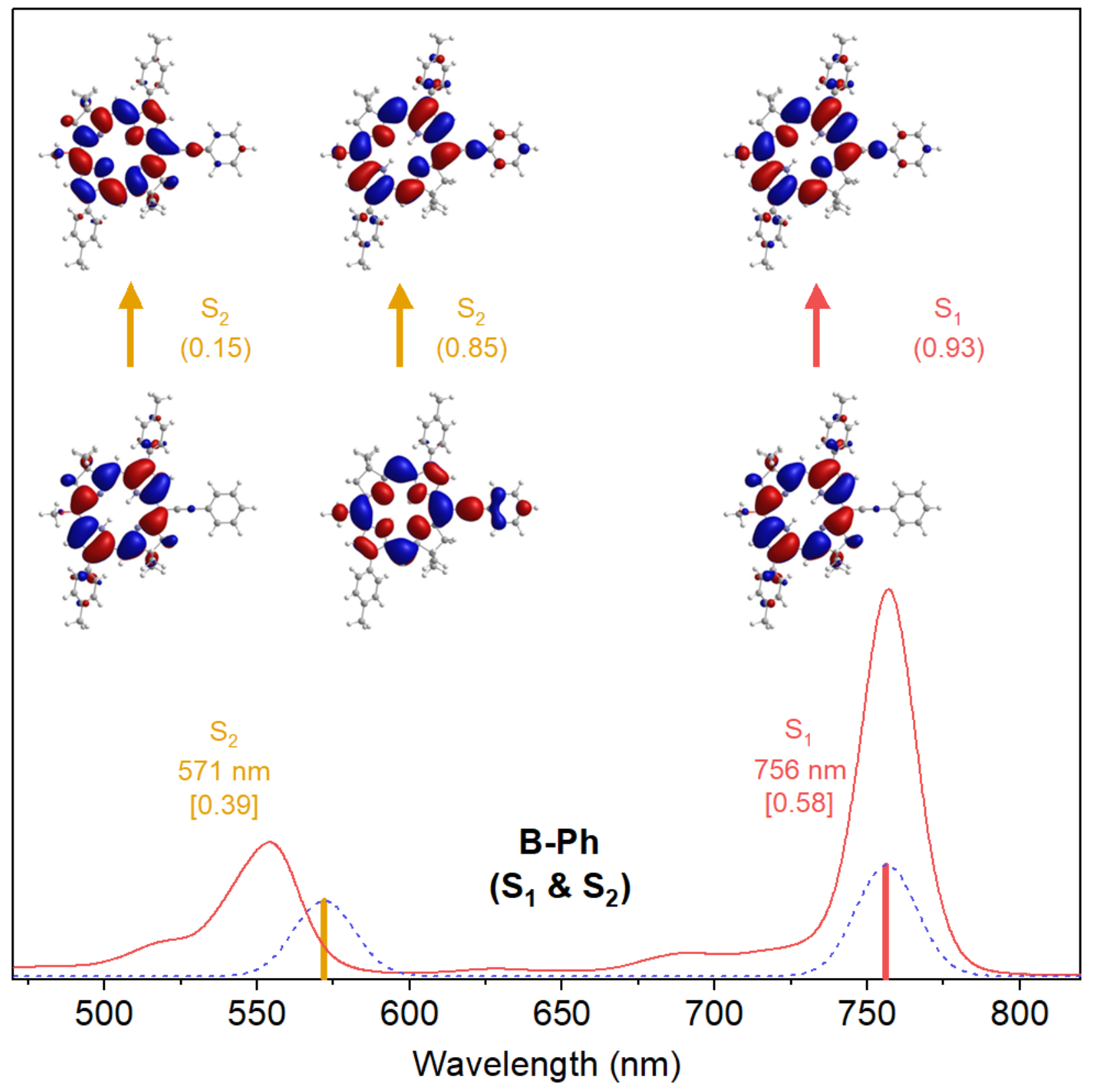

Figure S91. Comparison for benchmark B-Ph of the absorption spectra in toluene that are measured (red line) or calculated via TDDFT (dashed blue lines and colored sticks) normalized at the highest peak. The calculated spectrum was shifted arbitrarily by $0 \mathrm{~cm}^{-1}$ to lower energy to better align with the measured spectrum. Features in the calculated spectra were given 10-nm Gaussian skirts. The wavelength and oscillator strength (in square brackets) of the transitions from the ground state to $S_{1}$ (red) and $S_{2}$ (gold) calculated from TDDFT are indicated. Also shown are the sets of occupied and virtual NTOs along with the eigenvalue (weight) for each transition. 


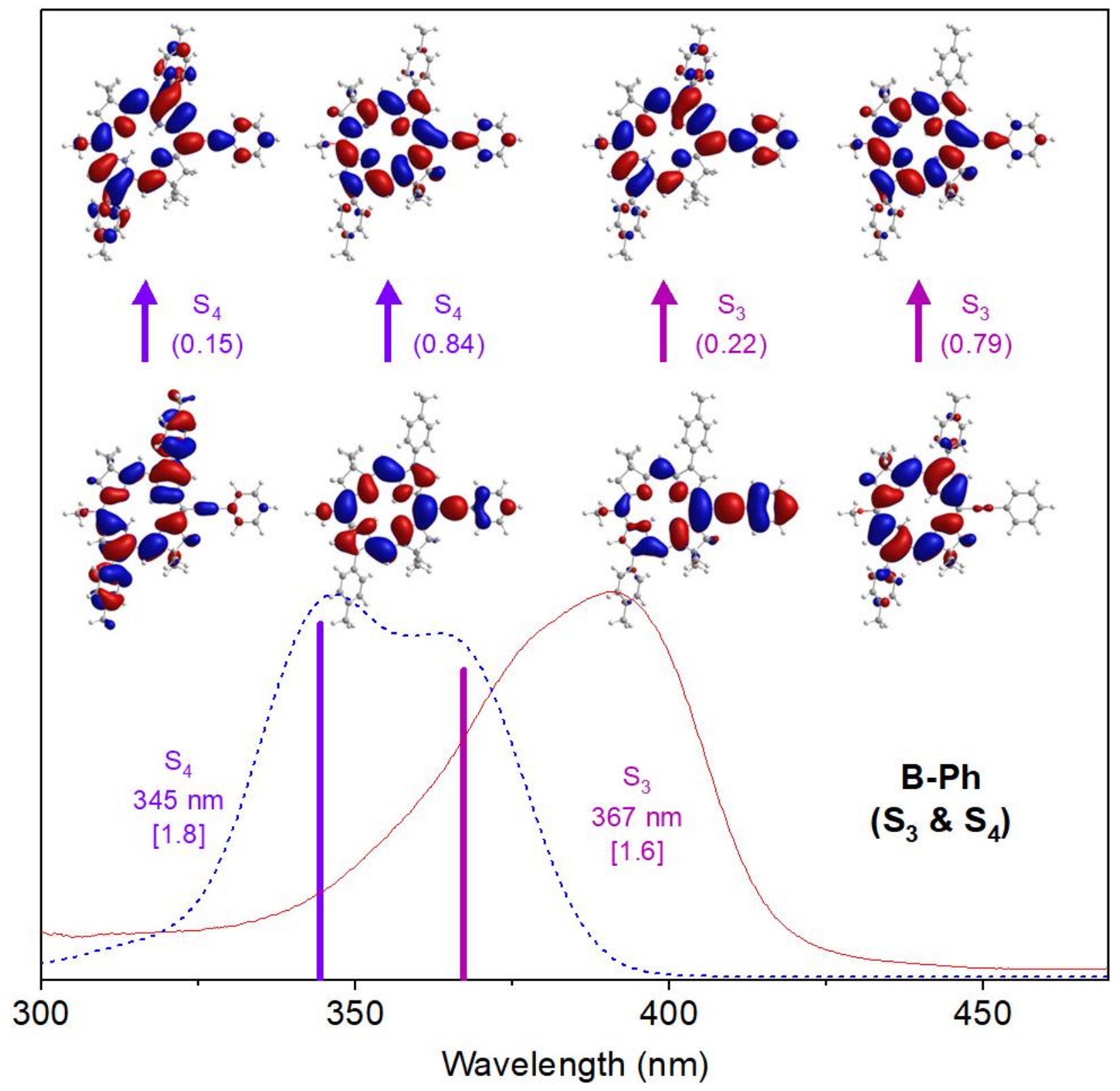

Figure S92. Comparison for benchmark B-Ph of the absorption spectra in toluene that are measured (red line) or calculated via TDDFT (dashed blue lines and colored sticks) normalized at the highest peak. The calculated spectrum was shifted arbitrarily by $0 \mathrm{~cm}^{-1}$ to lower energy to better align with the measured spectrum. Features in the calculated spectra were given 10-nm Gaussian skirts. The wavelength and oscillator strength (in square brackets) of the transitions from the ground state to $S_{3}$ (mauve) and $S_{4}$ (violet) calculated from TDDFT are indicated. Also shown are the sets of occupied and virtual NTOs along with the eigenvalue (weight) for each transition. 


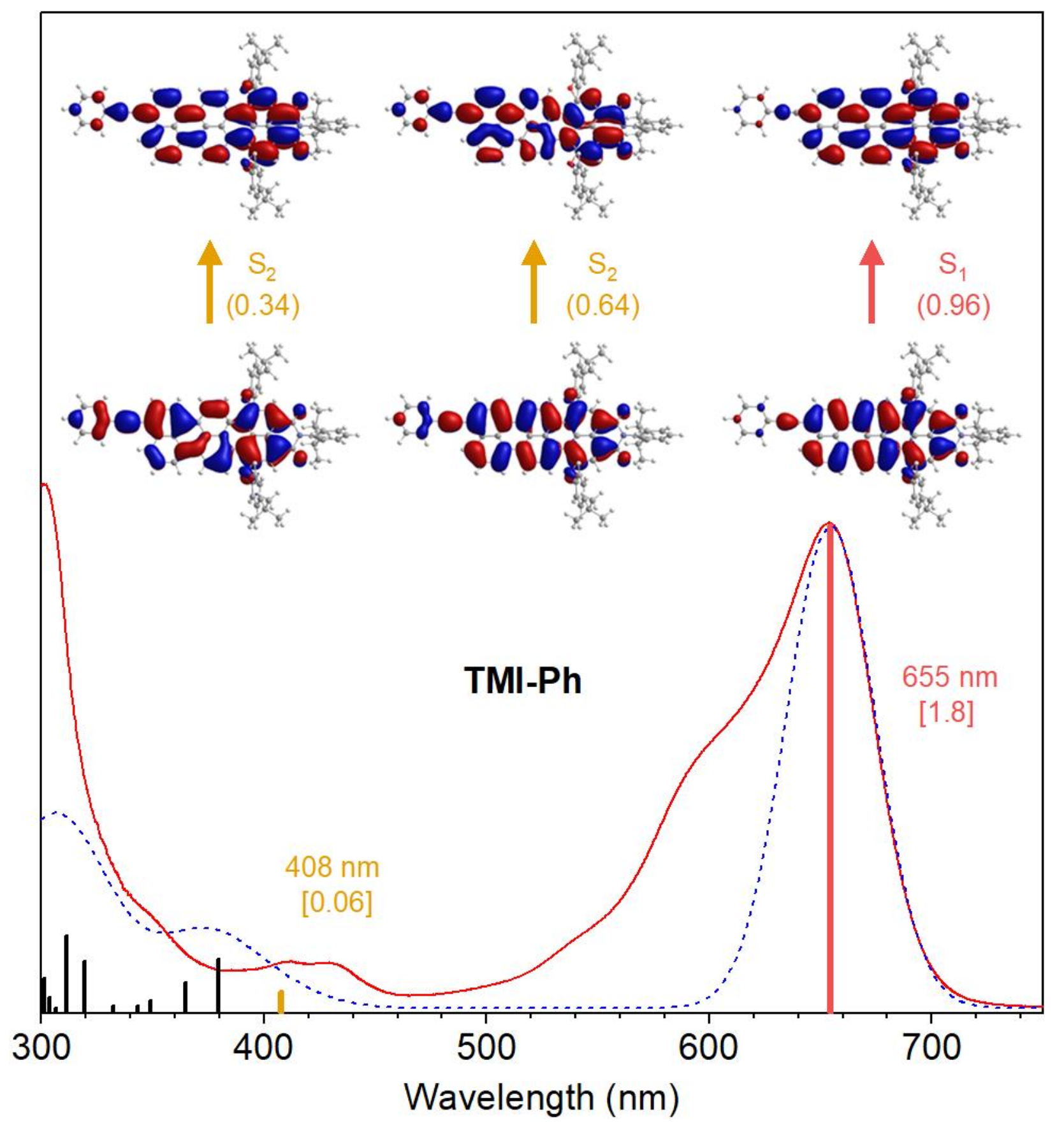

Figure S93. Comparison for benchmark TMI-Ph of the absorption spectra in toluene that are measured (red line) or calculated via TDDFT (dashed blue lines and colored sticks) normalized at the highest peak. The calculated spectrum was shifted arbitrarily by $1200 \mathrm{~cm}^{-1}$ to lower energy to better align with the measured spectrum. Features in the calculated spectra were given 20-nm Gaussian skirts. The wavelength and oscillator strength (in square brackets) of the transitions from the ground state to $S_{1}$ (red) and $S_{2}$ (gold) calculated from TDDFT are indicated. Also shown are the sets of occupied and virtual NTOs along with the eigenvalue (weight) for each transition. 


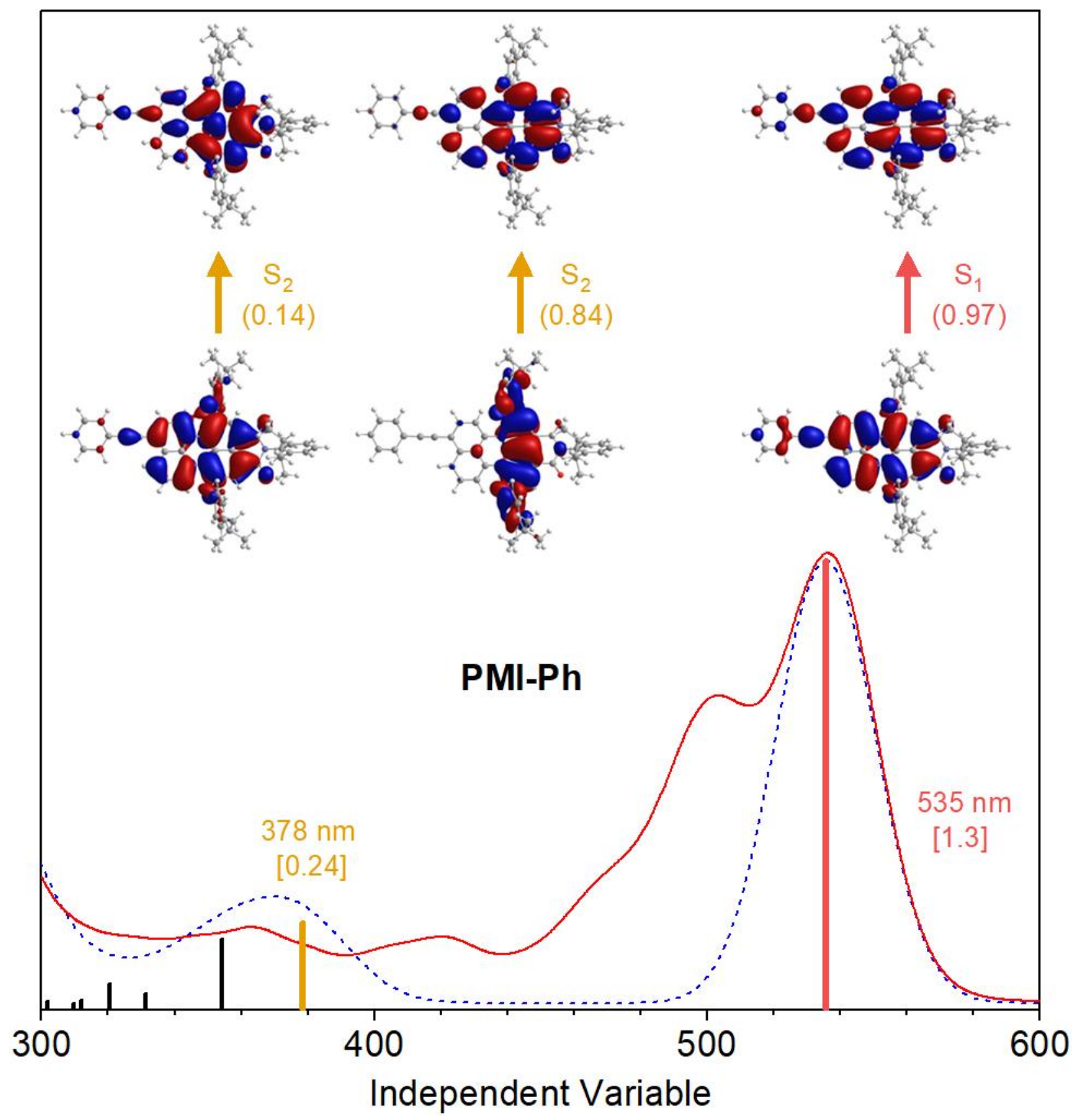

Figure S94. Comparison for benchmark PMI-Ph of the absorption spectra in toluene that are measured (red line) or calculated via TDDFT (dashed blue lines and colored sticks) normalized at the highest peak. The calculated spectrum was shifted arbitrarily by $1600 \mathrm{~cm}^{-1}$ to lower energy to better align with the measured spectrum. Features in the calculated spectra were given 15-nm Gaussian skirts. The wavelength and oscillator strength (in square brackets) of the transitions from the ground state to $S_{1}$ (red) and $S_{2}$ (gold) calculated from TDDFT are indicated. Also shown are the sets of occupied and virtual NTOs natural transition occupied and virtual orbitals along with the eigenvalue (weight) for each transition. 


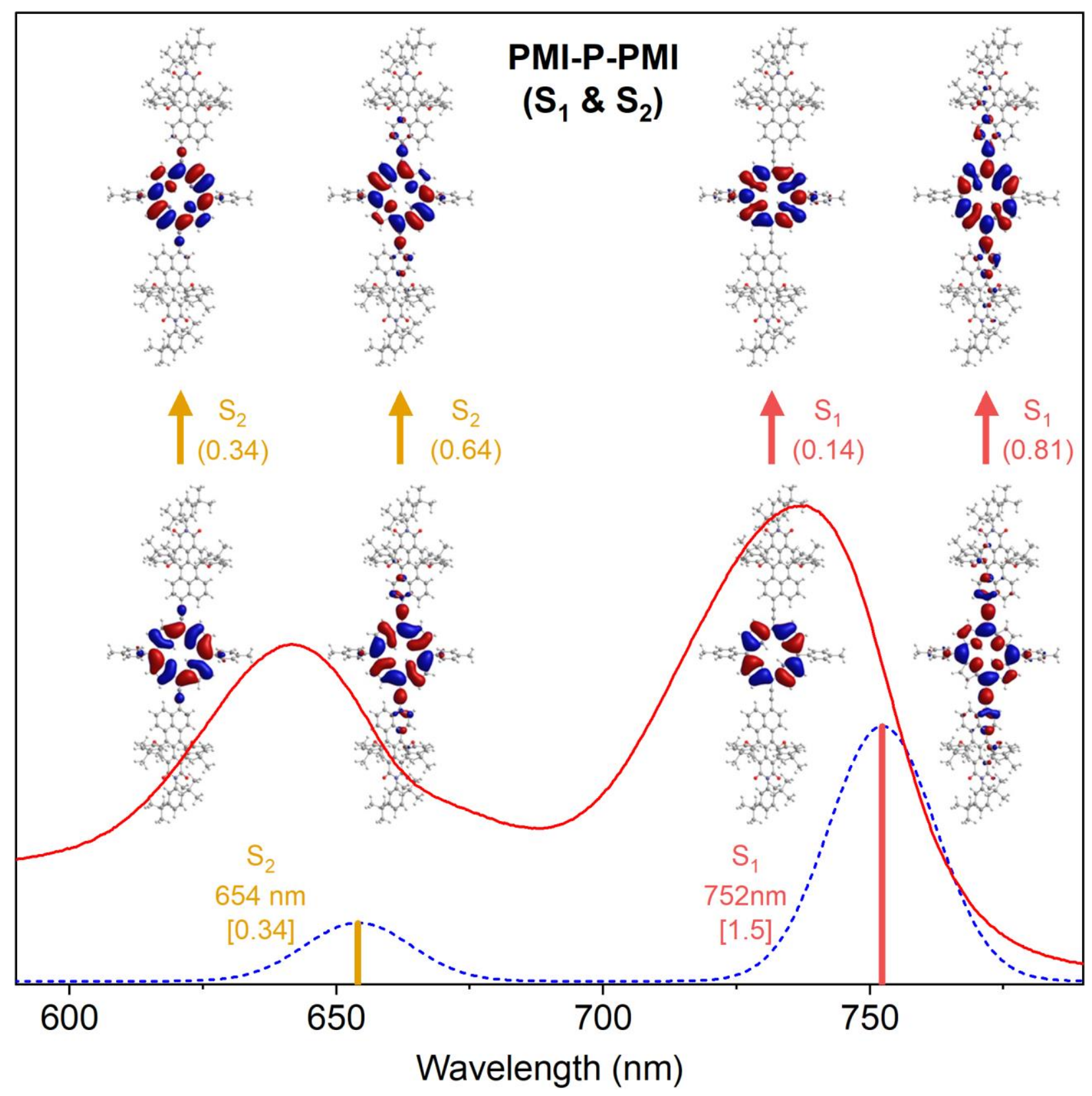

Figure S95. Comparison for triad PMI-P-PMI of the absorption spectra in toluene that are measured (red line) or calculated via TDDFT (dashed blue lines and colored sticks) normalized at the highest peak. The calculated spectrum was shifted arbitrarily by $800 \mathrm{~cm}^{-1}$ to lower energy to better align with the measured spectrum. Features in the calculated spectra were given 10-nm Gaussian skirts. The wavelength and oscillator strength (in square brackets) of the transitions from the ground state to $S_{1}$ (red) and $S_{2}$ (gold) calculated from TDDFT are indicated. Also shown are the sets of occupied and virtual NTOs along with the eigenvalue (weight) for each transition. 


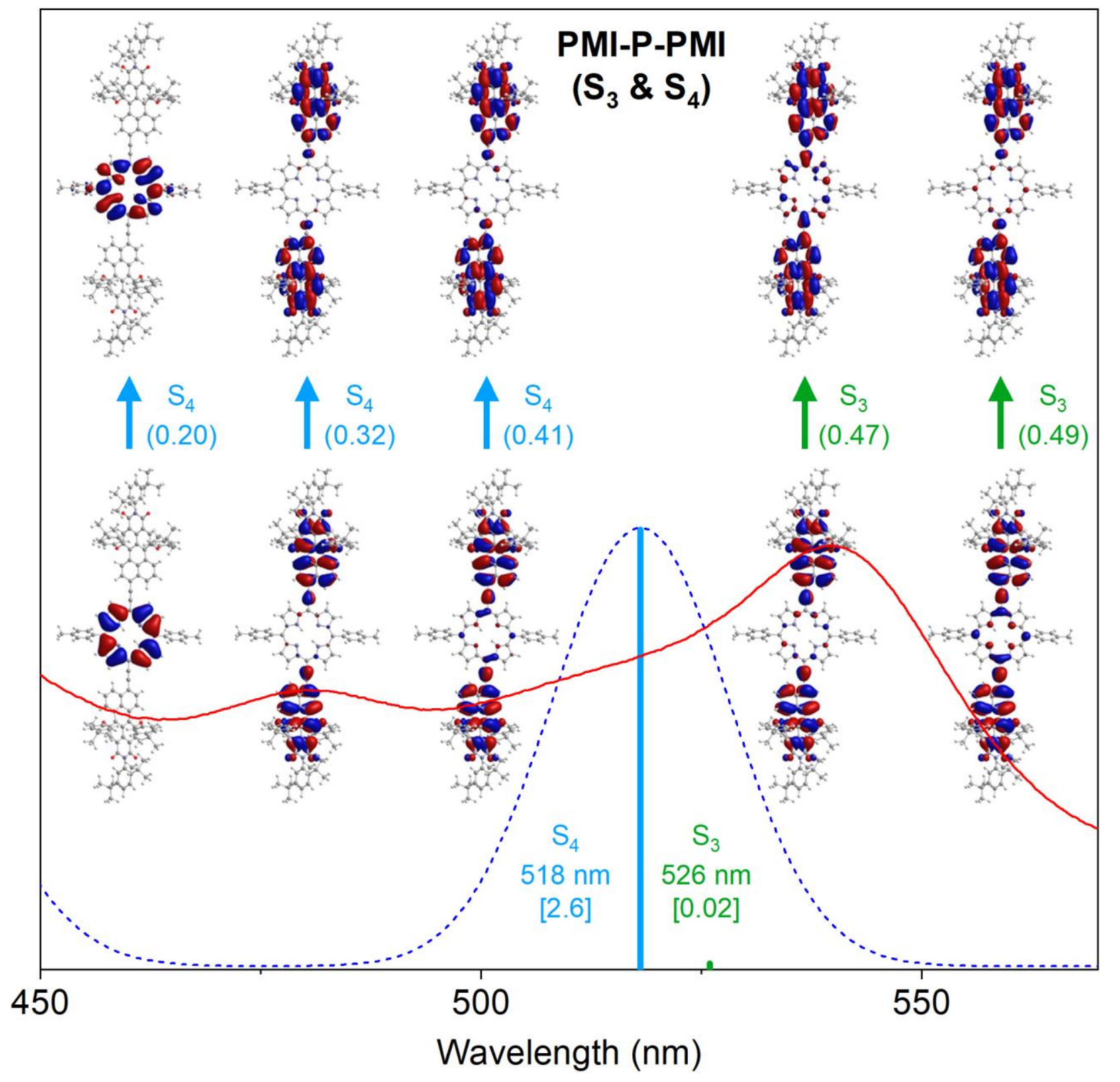

Figure S96. Comparison for triad PMI-P-PMI of the absorption spectra in toluene that are measured (red line) or calculated via TDDFT (dashed blue lines and colored sticks) normalized at the highest peak. The calculated spectrum was shifted arbitrarily by $800 \mathrm{~cm}^{-1}$ to lower energy to better align with the measured spectrum. Features in the calculated spectra were given 10-nm Gaussian skirts. The wavelength and oscillator strength (in square brackets) of the transition from the ground state to $\mathrm{S}_{3}$ (green) and $\mathrm{S}_{4}$ (blue) calculated from TDDFT is indicated. Also shown is the set of occupied and virtual NTOs along with the eigenvalue (weight) for the transitions. 


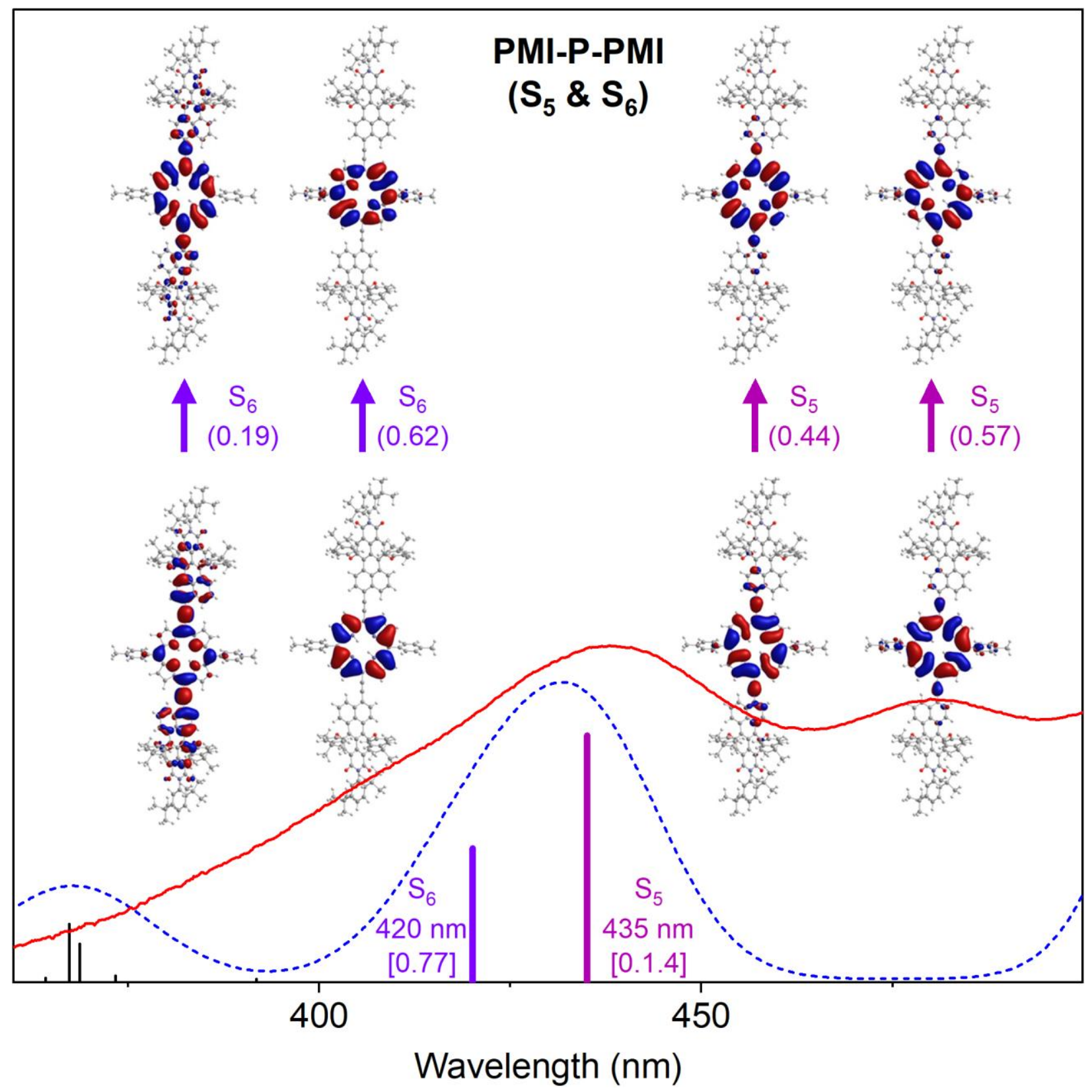

Figure S97. Comparison for triad PMI-P-PMI of the absorption spectra in toluene that are measured (red line) or calculated via TDDFT (dashed blue lines and colored sticks) normalized at the highest peak. The calculated spectrum was shifted arbitrarily by $800 \mathrm{~cm}^{-1}$ to lower energy to better align with the measured spectrum. Features in the calculated spectra were given 10-nm Gaussian skirts. The wavelength and oscillator strength (in square brackets) of the transitions from the ground state to $\mathrm{S}_{5}$ (mauve) and $\mathrm{S}_{6}$ (violet) calculated from TDDFT are indicated. Also shown are the sets of occupied and virtual NTOs along with the eigenvalue (weight) for each transition. 
Section 58

Calculated

Permanent

Dipoles 

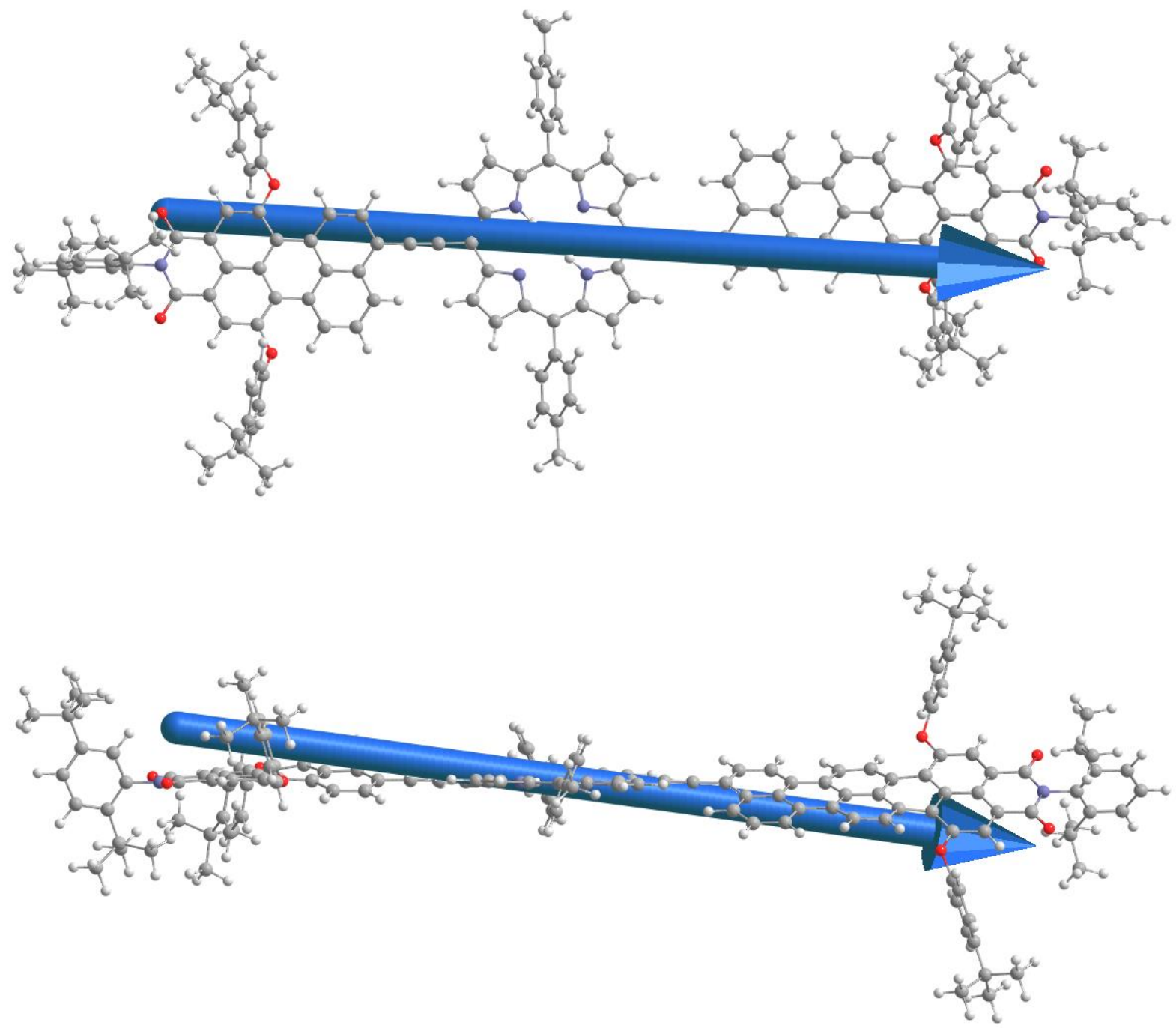

Figure S98. Two perspectives (face on or side on of the central porphyrin) of the permanent dipole moment for the ground state $\left(\mathrm{S}_{0}\right)$ of triad PMI-P-TMI, the value of which is 1.41 Debye. 

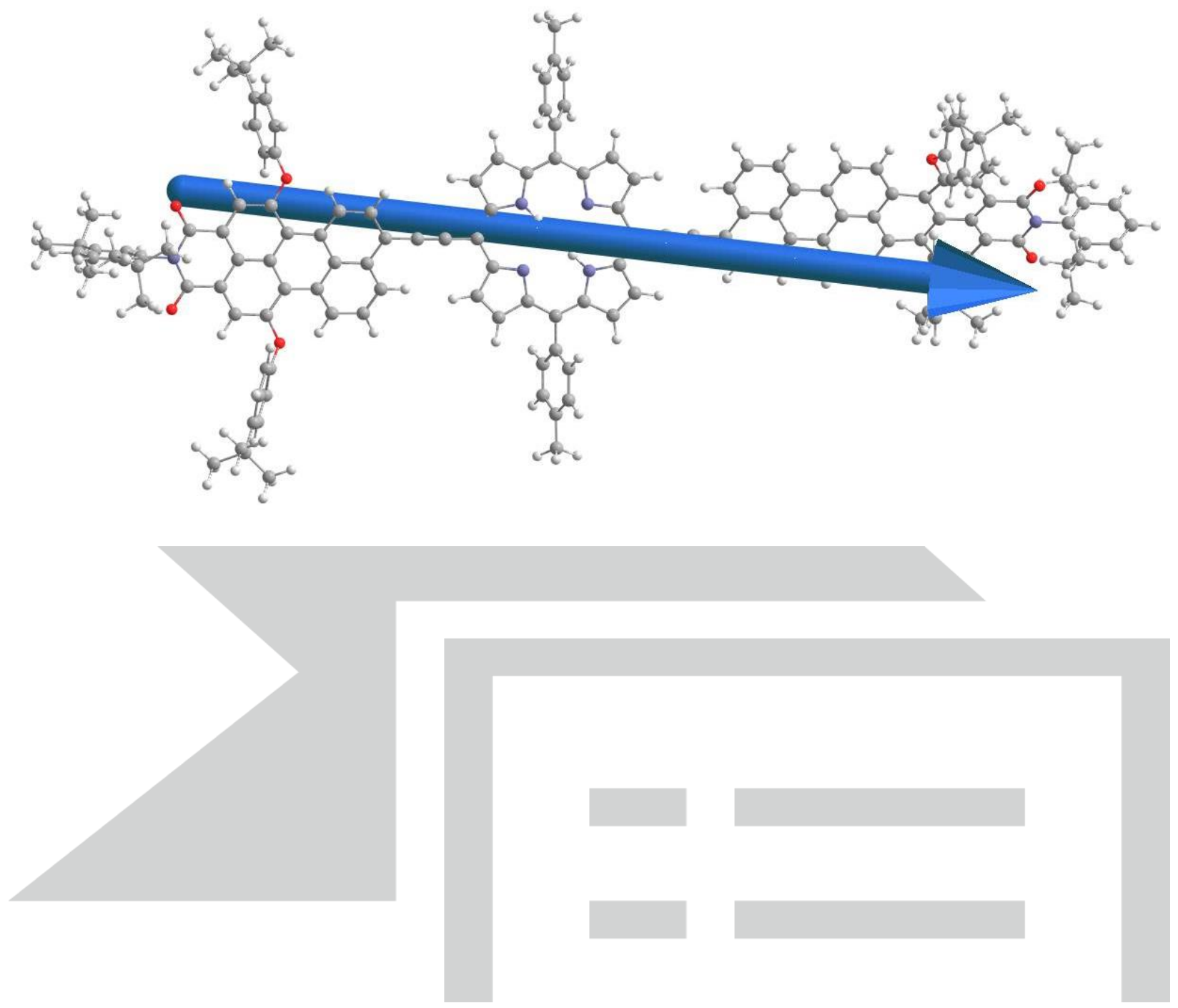

Figure S99. Two perspectives (face on or side on of the central porphyrin) of the permanent dipole moment for lowest singlet excited state $\left(\mathrm{S}_{1}\right)$ of triad PMI-P-TMI at the ground-state $\left(\mathrm{S}_{0}\right)$ geometry. The value is 1.25 Debye. 

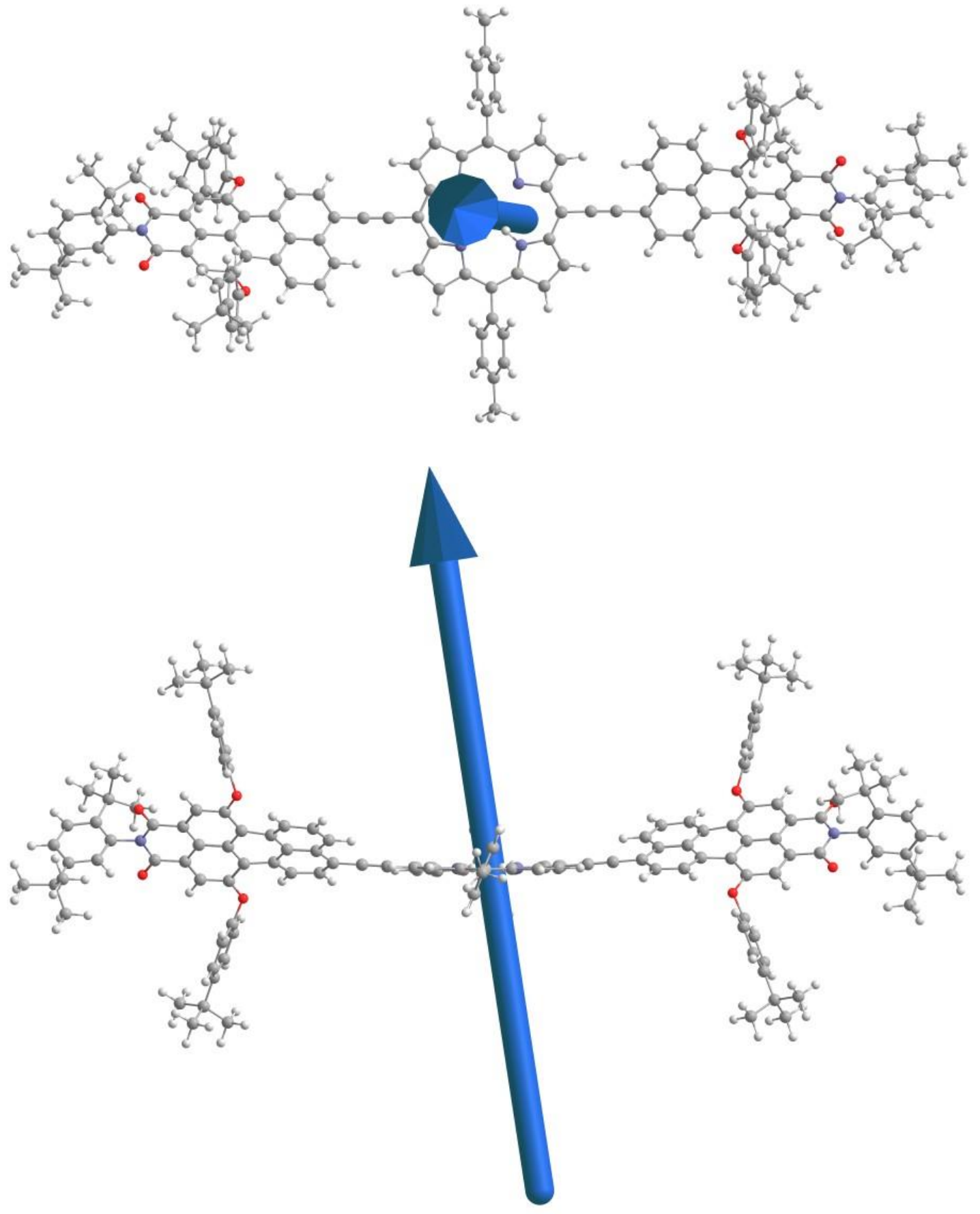

Figure S100. Two perspectives (face on or side on of the central porphyrin) of the permanent dipole moment for the ground state $\left(\mathrm{S}_{0}\right)$ of triad PMI-P-PMI, the value of which is 0.36 Debye. 

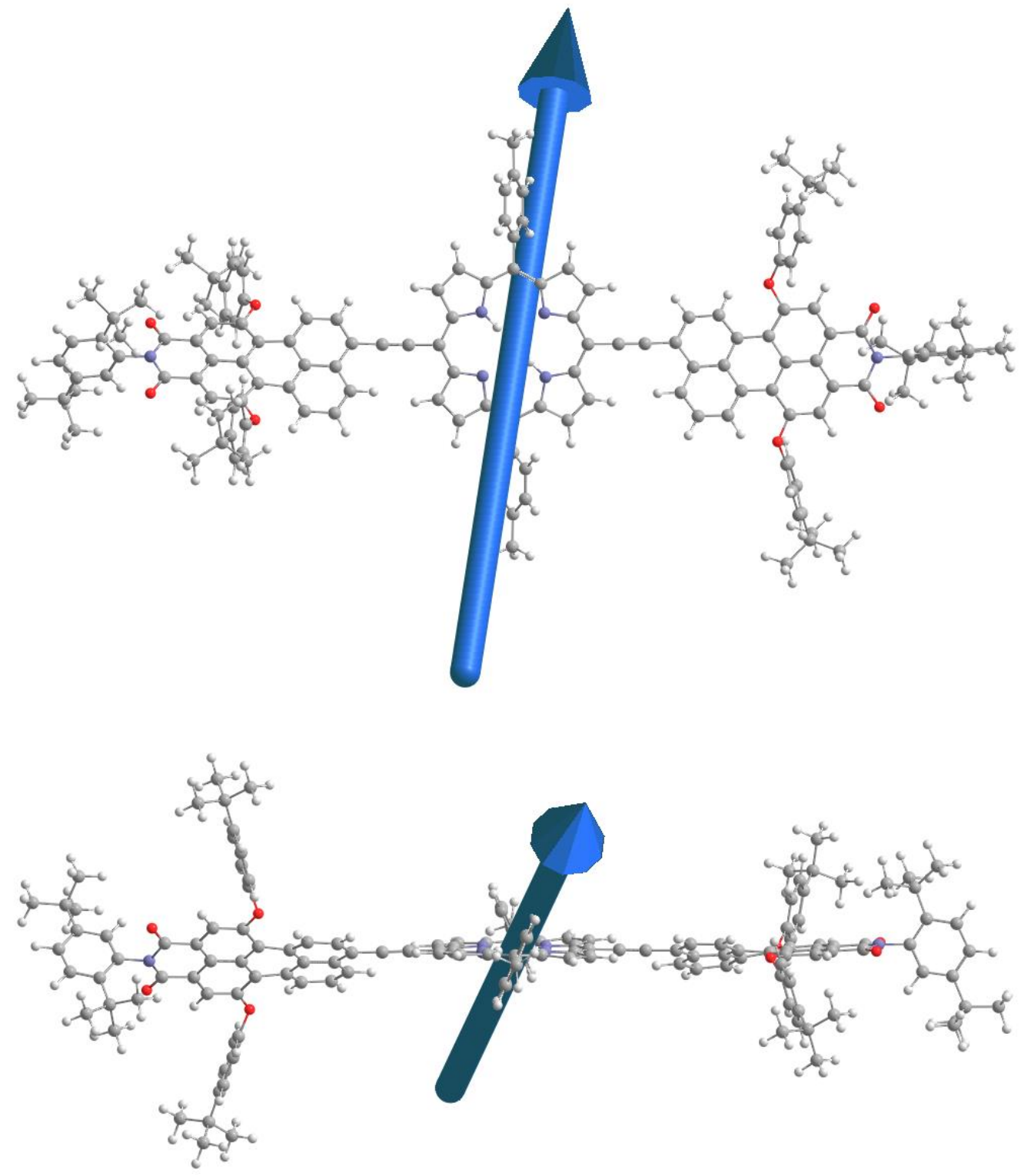

Figure S101. Two perspectives (face on or side on of the central porphyrin) of the permanent dipole moment for the lowest singlet excited state $\left(\mathrm{S}_{1}\right)$ of triad PMI-P-PMI at the ground-state $\left(\mathrm{S}_{0}\right)$ geometry. The value is 0.58 Debye. 


\section{Section S9}

Full References for Citations Truncated after the First 10
Authors 


\section{Full references}

(18) Yuen, J.; Diers, J. R.; Alexy, E. J.; Roy, A.; Mandal, A. K.; Kang, H. S.; Niedzwiedzki, D. M.; Kirmaier, C.; Lindsey, J. S.; Bocian, D. F.; Holten, D. Origin of Panchromaticity in Multichromophore-Tetrapyrrole Arrays. J. Phys. Chem. A 2018, 122, 7181-7201.

(26) Frisch, M. J.; Trucks, G. W.; Schlegel, H. B.; Scuseria, G. E.; Robb, M. A.; Cheeseman, J. R.; Scalmani, G.; Barone, V.; Mennucci, B.; Petersson, G. A.; Nakatsuji, H.; Caricato, M.; Li, X.; Hratchian, H. P.; Izmaylov, A. F.; Bloino, J.; Zheng, G.; Sonnenberg, J. L.; Hada, M.; Ehara, M.; Toyota, K.; Fukuda, R.; Hasegawa, J.; Ishida, M.; Nakajima, T.; Honda, Y.; Kitao, O.; Nakai, H.; Vreven, T.; Montgomery, J. A., Jr.; Peralta, J. E.; Ogliaro, F.; Bearpark, M.; Heyd, J. J.; Brothers, E.; Kudin, K. N.; Staroverov, V. N.; Kobayashi, R.; Normand, J.; Raghavachari, K.; Rendell, A.; Burant, J. C.; Iyengar, S. S.; Tomasi, J.; Cossi, M.; Rega, N.; Millam, J. M.; Klene, M.; Knox, J. E.; Cross, J. B.; Bakken, V.; Adamo, C.; Jaramillo, J.; Gomperts, R.; Stratmann, R. E.; Yazyev, O.; Austin, A. J.; Cammi, R.; Pomelli, C.; Ochterski, J. W.; Martin, R. L.; Morokuma, K.; Zakrzewski, V. G.; Voth, G. A.; Salvador, P.; Dannenberg, J. J.; Dapprich, S.; Daniels, A. D.; Farkas, Ö.; Foresman, J. B.; Ortiz, J. V.; Cioslowski, J.; Fox, D. J. Gaussian 09, version D.01; Gaussian, Inc., Wallingford CT, 2009.

(34) del Rosario Benites, M.; Johnson, T. E.; Weghorn, S.; Yu, L.; Rao, P. D.; Diers, J. R.; Yang, S. I.; Kirmaier, C.; Bocian, D. F.; Holten, D.; Lindsey, J. S. Synthesis and Properties of Weakly Coupled Dendrimeric Multiporphyrin Light-Harvesting Arrays and Hole-Storage Reservoirs. J. Mater. Chem. 2002, 12, 65-80.

(36) Kang, H. S.; Esemoto, N. N.; Diers, J. R.; Niedzwiedzki, D. M.; Greco, J. A.; Akhigbe, J.; Yu, Z.; Pancholi, C.; Bhagavathy, G. V.; Nguyen J. K.; Kirmaier, C.; Birge, R. R.; Ptaszek, M.; Holten, D.; Bocian, D. F. Effects of Strong Electronic Coupling in Chlorin and Bacteriochlorin Dyads. J. Phys. Chem. A 2016, 120, 379-395.

(43) Vairaprakash, P.; Yang, E.; Sahin, T.; Taniguchi, M.; Krayer, M.; Diers, J. R.; Wang, A.; Niedzwiedzki, D. M.; Kirmaier, C.; Lindsey, J. S.; Bocian, D. F.; Holten, D. Extending the Short and Long Wavelength Limits of Bacteriochlorin Near-Infrared Absorption via Dioxo- and Bisimide-Functionalization. J. Phys. Chem. B 2015, 119, 4382-4395.

(45) Li, F.; Yang, S. I.; Ciringh, Y.; Seth, J.; Martin III, C. H.; Singh, D. L.; Kim, D.; Birge, R. R.; Bocian, D. F.; Holten, D.; Lindsey, J. S. Design, Synthesis, and Photodynamics of Light-Harvesting Arrays Comprised of a Porphyrin and One, Two or Eight BoronDipyrrin Accessory Pigments. J. Am. Chem. Soc. 1998, 120, 10001-10017. 